\title{
SIX OPERATIONS ON DG ENHANCEMENTS OF DERIVED CATEGORIES OF SHEAVES
}

\author{
OLAF M. SCHNÜRER
}

\begin{abstract}
We lift Grothendieck-Verdier-Spaltenstein's six functor formalism for derived categories of sheaves on ringed spaces over a field to differential graded enhancements. Our main tools come from enriched model category theory.
\end{abstract}

\section{Contents}

1. Introduction

2. Description of the main results

3. Dg k-enriched functorial factorizations 17

4. Applications 24

5. Some 2-multicategories 37

6. Four operations 46

7. Two operations 67

8. The 2-multicategory of formulas 84

9. Some remarks on 2-morphisms in $\mathrm{ENH}_{\mathrm{k}} \quad 87$

Appendix A. Spaltenstein's results generalized to ringed topoi 93

Appendix B. Change of universe $\quad 97$

$\begin{array}{lll}\text { Appendix C. Some results on model categories } & 100\end{array}$

$\begin{array}{ll}\text { References } & 104\end{array}$

\section{INTRODUCTION}

1.1. Grothendieck-Verdier-Spaltenstein's six functor formalism in the topological setting concerns the six functors $\otimes^{\mathbf{L}}, \mathbf{R} \mathcal{H} o m, \mathbf{L} \alpha^{*}, \mathbf{R} \alpha_{*}, \mathbf{R} \alpha_{!}, \alpha^{!}$between derived categories of sheaves on ringed spaces and their relations [Ver66, Spa88, KS94, SS16]. Nowadays, triangulated categories are often replaced by suitable differential graded (dg) enhancements because some useful constructions can be performed with dg categories but not with triangulated categories [BK90, Kel06]. Therefore it is natural to ask whether Grothendieck-Verdier-Spaltenstein's six functor formalism lifts to the level of $\mathrm{dg}$ enhancements. We give an affirmative answer to this question if we fix a field $\mathrm{k}$ and work with $\mathrm{k}$-ringed spaces, i. e. pairs $(X, \mathcal{O})$ consisting of a topological space $X$ and a sheaf $\mathcal{O}$ of commutative k-algebras on $X$. Here is our main result. 
Theorem 1.1. Let k be a field. Then Grothendieck-Verdier-Spaltenstein's six functor formalism for $\mathrm{k}$-ringed spaces lifts to $\mathrm{dg} \mathrm{k}$-enhancements. The formalism involving the first four functors lifts more generally for k-ringed topoi.

Our main tools to lift the six functors come from enriched model category theory. These tools are not applicable if we work over the integers. More details and the precise meaning of this theorem are explained later on in this introduction.

Our original interest in this lifting problem arose when we studied the relation between geometric and homological smoothness and translated Fourier-Mukai functors to the dg world [Lun10, LS16]. For the arguments given there and in many other instances it is necessary to lift certain isomorphisms from the triangulated level to the dg level. Our dg enhanced six functor formalism provides a solution to this lifting problem, see section 2.2 .

The need to address lifting problems as mentioned above is well-known to the experts. For example, Drinfeld writes in the introduction to [Dri08]: "Hopefully the part of homological algebra most relevant for algebraic geometry will be rewritten using dg categories or rather the more flexible notion of $A_{\infty}$-category." In this article we mostly work in the topological setting. However, our techniques can be extended to many other contexts. For example, they can be used to lift the formalism involving the various derived categories associated to schemes (or algebraic stacks) over a field $k$ to dg k-enhancements. Presumably, more details will appear in forthcoming articles. Some preparatory results concerning modules over dg k-categories and, in particular, modules over k-algebras are already given here.

On the higher categorical level, six functor formalisms have been considered in the context of stable $\infty$-categories by Liu and Zheng [LZ17] and in the context of derivators by Hörmann [Hör17]. The notion of a derived functor in the dg setting is discussed by Drinfeld [Dri08]. To the best of our knowledge, however, a six functor formalism in the context of $\mathrm{dg}$ categories has not been established elsewhere in the literature. Based on Weibel's results [Wei89, App.], one out of the six functors, the derived inverse image, is lifted to $\mathrm{dg}$ enhancements by Cirone [Cir17]. There are several ad hoc constructions lifting certain morphisms from the triangulated level to the dg level, see e.g. [Gui11, Kuz15, LS16, PvdB17]. Let us also mention the claim without proof in [Nad09, 2.2] that the six functor formalism can be lifted to dg enhancements.

1.2. Grothendieck-Verdier-Spaltenstein's six functor formalism takes place in the klinear 2-multicategory TRCAT $_{\mathrm{k}}$ of triangulated k-categories (the prefix "multi" takes care of functors with several inputs like $\otimes^{\mathbf{L}}$ and $\mathbf{R} \mathcal{H}$ om; $\mathbf{k}$-linearity refers to the k-vector spaces of natural transformations). The relevant objects are the derived categories $\mathrm{D}(X)$ of sheaves of $\mathcal{O}$-modules on $X$. The six functors $\otimes^{\mathbf{L}}, \mathbf{R} \mathcal{H}$ om, $\mathbf{L} \alpha^{*}$, $\mathbf{R} \alpha_{*}, \mathbf{R} \alpha_{!}, \alpha^{!}$are 1-morphisms between these objects. The relations between these 
functors are encoded in two ways: first, by 2-morphisms like id $\rightarrow \mathbf{R} \alpha_{*} \mathbf{L} \alpha^{*}$ and 2-isomorphisms like $\mathbf{R} \alpha_{*} \mathbf{R} \mathcal{H o m}\left(-, \alpha^{!}(-)\right) \stackrel{\sim}{\rightarrow} \mathbf{R} \mathcal{H o m}\left(\mathbf{R} \alpha_{!}(-),-\right)$; second, by commutative diagrams constructed from these 2-morphisms (i. e. by equalities between compositions of 2-morphisms): they encode for example that $\left(\mathbf{L} \alpha^{*}, \mathbf{R} \alpha_{*}\right)$ is a pair of adjoint functors or that $\left(\mathrm{D}(X), \otimes^{\mathbf{L}}\right)$ is a symmetric monoidal category.

Ideally, one would hope to find a corresponding formalism in some subcategory of the k-linear 2-multicategory $\mathrm{DGCAT}_{\mathrm{k}}$ of $\mathrm{dg}$ k-categories. We achieve this goal up to inverting some 2-morphisms as follows.

We define a k-linear 2-multicategory $\mathrm{ENH}_{\mathrm{k}}$ of $\mathrm{dg}$ k-enhancements. Its objects are additive pretriangulated $\mathrm{dg}$ k-categories, its 1-morphisms coincide with the corresponding 1-morphisms in $\mathrm{DGCAT}_{\mathrm{k}}$, i.e. with dg k-functors, and its 2-morphisms can be represented by zig-zags of 2-morphisms in $\mathrm{DGCAT}_{k}$, i. e. by zig-zags of honest dg k-natural transformations, where the arrows pointing in the wrong direction are objectwise homotopy equivalences (see section 2.1.3). More precisely, the morphism categories of $\mathrm{ENH}_{\mathrm{k}}$ are obtained from the corresponding morphism categories of $\mathrm{DGCAT}_{\mathrm{k}}$ by inverting the objectwise homotopy equivalences. Mapping a dg kcategory $\underline{\mathcal{N}}$ to its homotopy category $[\underline{\mathcal{N}}]$ defines a functor

$$
[-]: \mathrm{ENH}_{\mathrm{k}} \rightarrow \mathrm{TRCAT}_{\mathrm{k}} \text {. }
$$

We establish a six functor formalism in $\mathrm{ENH}_{\mathrm{k}}$ and show that it corresponds to Grothendieck-Verdier-Spaltenstein's six functor formalism under the functor (1.1). The objects of $\mathrm{ENH}_{\mathrm{k}}$ that enhance the derived categories $\mathrm{D}(X)$ are the dg $\mathrm{k}$-categories $\underline{I}(X)$ of h-injective complexes of injective sheaves on $X$ : the homotopy category $[\underline{I}(X)]$ of $\mathbb{I}(X)$ is equivalent to $\mathrm{D}(X)$. The 1-morphisms in $\mathrm{ENH}_{\mathrm{k}}$ that enhance the six functors $\otimes^{\mathbf{L}}, \mathbf{R} \mathcal{H} o m, \mathbf{L} \alpha^{*}, \mathbf{R} \alpha_{*}, \mathbf{R} \alpha_{!}, \alpha^{!}$are dg k-functors $\underline{\otimes}, \underline{\mathcal{H} o m}, \underline{\alpha}^{*}, \underline{\alpha}_{*}, \underline{\alpha}_{!}$, $\underline{\alpha}^{!}$. Their definition relies on the fact that $\mathrm{k}$ is a field. We lift all the standard 2(iso)morphisms in TRCAT $_{k}$ in the right columns of tables 1 and 2 on pages 6 and 7 to 2-(iso)morphisms in $\mathrm{ENH}_{\mathrm{k}}$ in the middle columns of these tables. All these lifts are defined by explicit zig-zags of 2-morphisms in $\mathrm{DGCAT}_{\mathrm{k}}$; the lifts marked as 2-isomorphisms come from zig-zags of objectwise homotopy equivalences (with one exception, namely the 2-isomorphism $\underline{\beta}_{*}^{\prime} \underline{\alpha}^{\prime} \stackrel{\sim}{\rightarrow} \underline{\alpha}^{!} \underline{\beta}_{*}$ in row (T2.8) of table 2). We prove commutativity of certain diagrams constructed from these lifts (see section 1.7 for some of these diagrams); for example we show that $\left(\underline{\alpha}^{*}, \underline{\alpha}_{*}\right)$ forms a pair of adjoint 1-morphisms and that $(\underline{\mathbb{I}}(X), \underline{\otimes})$ is a symmetric monoidal object of $\mathrm{ENH}_{\mathrm{k}}$.

We encourage the reader to look at section 2 where we give a very precise description of our results. In section 2.1 we give a user's guide to the dg k-enhanced six functor formalism. We also describe the k-linear 2-multicategory $\mathrm{FML}_{\mathrm{k}}$ of formulas there (see section 2.1.8). It is our main formal tool to describe all relations between functors between derived categories of sheaves that we can lift to dg k-enhancements. We use it to explain the precise meaning of Theorem 1.1 (see Theorem 2.1 and section 2.1.9). In section 2.2 we explain how to lift certain isomorphisms from the triangulated level 
to the dg level. We then comment on the ingredients from enriched model category theory in section 2.3. Let us also mention Lemma 4.4, which explains why we need to work over a field.

1.3. Our article contains three appendices. The first two appendices contain foundational statements of independent interest. In appendix A we extend some results of Spaltenstein [Spa88] from ringed spaces to ringed topoi. In appendix B we explain that passing to a higher Grothendieck universe yields fully faithful embeddings on derived categories of sheaves; more precisely, we show that the change of Grothendieck universe functor preserves h-injective complexes of sheaves. Surprisingly, these results seem not to be available elsewhere in the literature.

1.4. Plan of the article. We consider section 2 described above as an extended introduction. The aim of section 3 is to formulate and prove Theorem 3.6. It provides criteria to ensure that a model structure on a dg k-category admits dg k-enriched functorial factorizations. This result is applied in section 4 where we consider various model structures on categories of complexes of sheaves and on categories of $\mathrm{dg}$ modules and show that they are compatible with the $\mathrm{dg}$ k-enrichments of these categories. The k-linear 2-multicategory $\mathrm{ENH}_{\mathrm{k}}$ of dg k-enhancements is defined in section 5. We then lift the six functor formalism: the four functors $\otimes^{\mathbf{L}}, \mathbf{R} \mathcal{H} o m, \mathbf{L} \alpha^{*}, \mathbf{R} \alpha_{*}$ associated to k-ringed topoi and their morphisms are treated in section 6 ; the two functors $\mathbf{R} \alpha_{\text {! }}$, $\alpha^{!}$associated to suitable morphisms of k-ringed spaces (cf. [SS16]) are included into the picture in section 7. The k-linear 2-multicategory of formulas $\mathrm{FML}_{\mathrm{k}}$ is defined and used in section 8 to state the summarizing Theorem 8.5. In section 9 we explain some useful facts concerning 2-morphisms in $\mathrm{ENH}_{\mathrm{k}}$; for example, we provide some instances where such 2-morphisms can be represented by roofs of 2-morphisms in DGCAT $_{k}$ where the arrow pointing in the wrong direction is an objectwise homotopy equivalence. In three appendices we prove some facts we could not locate in the literature. The content of the appendices A and B was already discussed in section 1.3. Some basic model categorical facts are proved in appendix C.

The results of the two appendices $\mathrm{B}$ and $\mathrm{C}$ are only used in section 9 . This section is not used elsewhere in this article, and the reader is advised to skip it on a first reading. This also has the advantage that Grothendieck universes and related set-theoretical issues can be safely ignored.

1.5. Acknowledgements. We thank Valery Lunts for many inspiring discussions. He was hoping very much that a theory as presented in this work should exist. We thank Michael Mandell for discussions and Emily Riehl and Michael Shulman for useful correspondence concerning model categories. We thank Timothy Logvinenko, Hanno Becker, Alexander Efimov, James Gillespie, Greg Stevenson, PierreYves Gaillard, Lorenzo Ramero and Amnon Neeman for useful discussions. Hanno Becker and Jan Weidner shared an observation which led to Lemma 4.4. Frédéric 
Déglise answered a question concerning Theorem 4.8. We thank the referee for very detailed comments, in particular for drawing our attention to set-theoretical problems concerning functor categories, and for suggesting a more intrinsic definition of the 2-multicategory $\mathrm{ENH}_{\mathrm{k}}$ of $\mathrm{dg}$ enhancements.

The author was supported by a postdoctoral fellowship of the DFG, and by SPP 1388 and SFB/TR 45 of the DFG.

1.6. Conventions. All rings considered are assumed to be associative and unital. The symbol $\mathrm{R}$ always denotes a commutative ring, and $\mathrm{k}$ always denotes a field. We often write $\otimes$ instead of $\otimes_{\mathrm{R}}$ or $\otimes_{\mathrm{k}}$ or $\otimes_{\mathcal{O}_{X}}$ and hope that its meaning is clear from the context.

Starting from 3.1.1 we use the notation $\mathcal{R}$ for the category of complexes of $\mathrm{R}$ modules (in some Grothendieck universe) and $\underline{\mathcal{R}}$ for the $\mathrm{dg}$ R-category of such complexes. Similarly, $\mathcal{K}$ denotes the category of complexes of k-vector spaces and $\underline{\mathcal{K}}$ the dg k-category of complexes of k-vector spaces.

Starting from 3.2 we will mostly use the term $\mathcal{R}$-category for a category enriched in $\mathcal{R}$. This is just another name for dg R-category. So $\underline{\mathcal{R}}$ is an $\mathcal{R}$-category. Similarly, we will mostly say $\mathcal{K}$-category instead of dg k-category. So $\underline{\mathcal{K}}$ is a $\mathcal{K}$-category.

1.6.1. Set-theoretical conventions. We use (Grothendieck) universes in order to handle set-theoretical issues [SGA4i, KS06]. These issues are most relevant in section 9; the reader can safely ignore them before reading this section. To be more precise, we work in a model of Tarski-Grothendieck set theory. All universes we consider are assumed to contain the set $\mathbb{N}$ of natural numbers, the ring $\mathrm{R}$ and the field $\mathrm{k}$ as elements.

Given a universe $\mathcal{U}$, we use the following terminology (which follows [GR17] but differs from [SGA4i]): a set (or an algebraic structure like a module over some ring) is called $\mathcal{U}$-small if it is an element of $\mathcal{U}$; a category $\mathcal{C}$ has $\mathcal{U}$-small Hom-sets if $\mathcal{C}(A, B)$ is $\mathcal{U}$-small for all objects $A, B \in \mathcal{C}$; a category $\mathcal{C}$ is $\mathcal{U}$-small if it has $\mathcal{U}$-small Hom-sets and if its set $\mathrm{Obj} \mathcal{C}$ of objects is $\mathcal{U}$-small; a category $\mathcal{C}$ has objects in $\mathcal{U}$ if $\operatorname{Obj} \mathcal{C}$ is a subset of $\mathcal{U}$.

We use the convention of [GR17, Rem. 1.1.12.(iii)] that a map $f: S \rightarrow S^{\prime}$ of sets is given by its graph (and not by the triple consisting of $S, S^{\prime}$ and its graph); this has the small technical advantage that Remark 3.2 is true. Similarly, we do not assume that the Hom-sets of a category $\mathcal{C}$ are disjoint; nevertheless we refer to $\operatorname{Mor}(\mathcal{C}):=\{(A, B, f) \mid A, B \in \operatorname{Obj} \mathcal{C}, f \in \mathcal{C}(A, B)\}$ as the set of morphisms in $\mathcal{C}$ (with source and target remembered).

We try to mention size issues whenever relevant. This increase in correctness hopefully makes up for some decrease in readability. 
TABLE 1. Interpretation of 2-morphisms in $\mathrm{FML}_{\mathrm{k}}$ in $\mathrm{ENH}_{\mathrm{k}}$ and $\mathrm{TRCAT}_{\mathrm{k}}$

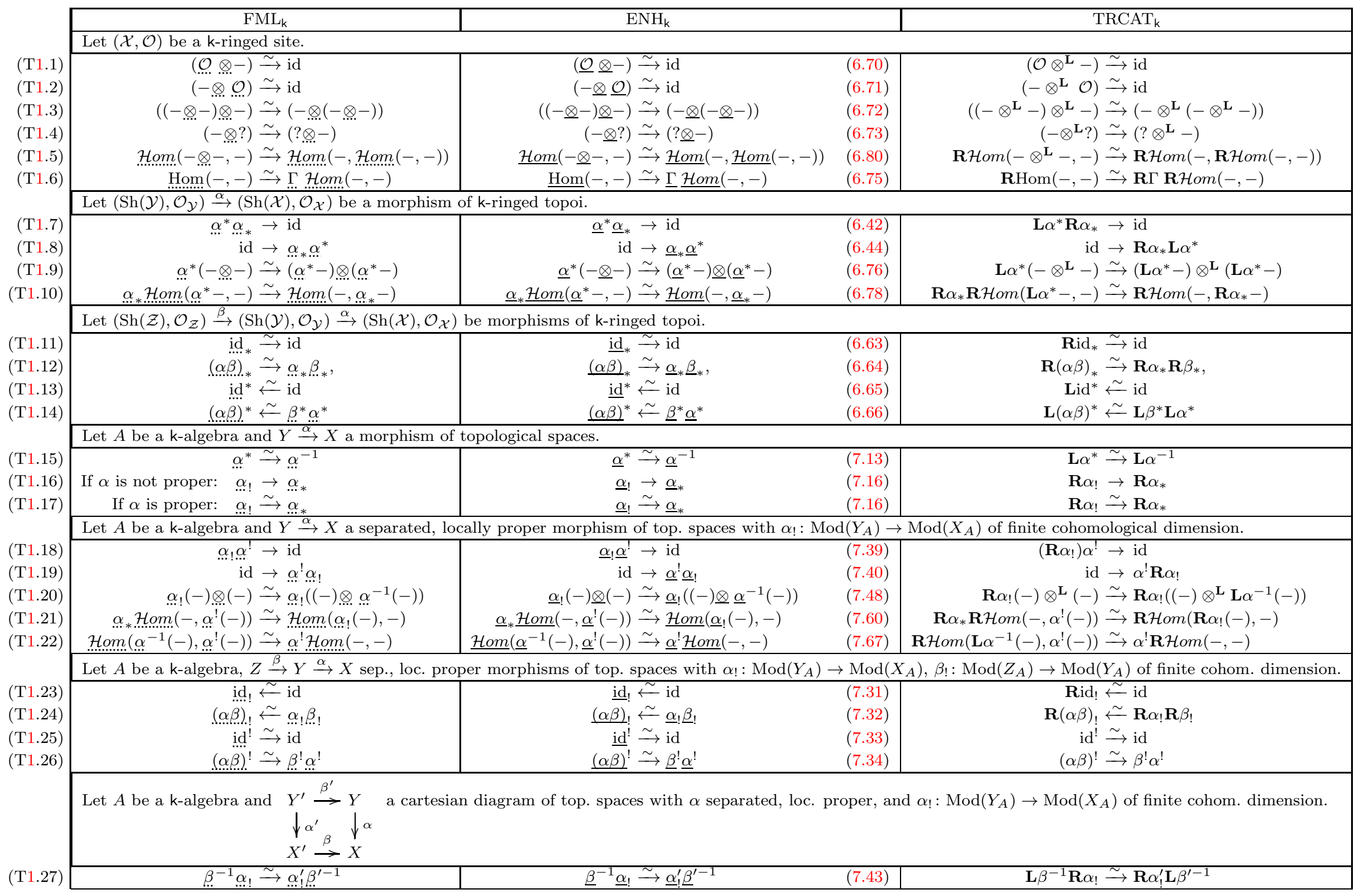


TABLE 2. Some subsequently constructed 2-(iso)morphisms and their interpretation

\begin{tabular}{|c|c|c|c|c|}
\hline \multirow{4}{*}{ (T2.1) } & $\mathrm{FML}_{\mathrm{k}}$ & $\mathrm{ENH}_{\mathrm{k}}$ & & $\mathrm{TRCAT}_{\mathrm{k}}$ \\
\hline & \multicolumn{4}{|l|}{ Let $(\mathcal{X}, \mathcal{O})$ be a k-ringed site. } \\
\hline & $\operatorname{Hom}(-\otimes-,-,) \stackrel{\sim}{\longrightarrow} \operatorname{Hom}(-, \mathcal{H o m}(-,-))$ & $\underline{\operatorname{Hom}}(-\underline{\underline{Q}}-,-) \stackrel{\sim}{\longrightarrow} \underline{\operatorname{Hom}}(-, \underline{\mathcal{H o m}}(-,-))$ & $(6.105)$ & $\mathbf{R H o m}\left(-\otimes^{\mathbf{L}}-,-\right) \stackrel{\sim}{\longrightarrow} \mathbf{R H o m}(-, \mathbf{R} \mathcal{H o m}(-,-))$ \\
\hline & \multicolumn{4}{|c|}{ Let $\left(\operatorname{Sh}(\mathcal{Y}), \mathcal{O}_{\mathcal{Y}}\right) \stackrel{\alpha}{\rightarrow}\left(\operatorname{Sh}(\mathcal{X}), \mathcal{O}_{\mathcal{X}}\right)$ be a morphism of k-ringed topoi. } \\
\hline$(\mathrm{T} 2.2)$ & $\operatorname{Hom}\left(\alpha^{*}-,-\right) \stackrel{\sim}{\rightarrow} \operatorname{Hom}\left(-, \underline{\alpha}_{*}-\right)$ & $\underline{\operatorname{Hom}}\left(\underline{\alpha}^{*}-,-\right) \stackrel{\sim}{\longrightarrow} \underline{\operatorname{Hom}}\left(-, \underline{\alpha}_{*}-\right)$ & $(6.102)$ & $\mathbf{R H o m}\left(\mathbf{L} \alpha^{*}-,-\right) \stackrel{\sim}{\longrightarrow} \mathbf{R H o m}\left(-, \mathbf{R} \alpha_{*}-\right)$ \\
\hline \multirow[t]{2}{*}{ (T2.3) } & $\alpha, \mathcal{H} o m(-,-) \rightarrow \mathcal{H o m}\left(\alpha_{* *}(-), \alpha_{* *}(-)\right)$ & $\underline{\alpha}_{*} \underline{\mathcal{H} o m}(-,-) \rightarrow \underline{\mathcal{H} o m}\left(\underline{\alpha}_{*}(-), \underline{\alpha}_{*}(-)\right)$ & $(6.106)$ & $\mathbf{R} \alpha_{*} \mathbf{R} \mathcal{H o m}(-,-) \rightarrow \mathbf{R} \mathcal{H o m}\left(\mathbf{R} \alpha_{*}(-), \mathbf{R} \alpha_{*}(-)\right)$ \\
\hline & \multicolumn{4}{|c|}{ Let $A$ be a k-algebra and $Y \stackrel{\alpha}{\rightarrow} X$ a morphism of topological spaces. } \\
\hline \multirow[t]{2}{*}{$\begin{array}{l}(\mathrm{T} 2.4) \\
(\mathrm{T} 2.5)\end{array}$} & $\begin{aligned} \alpha^{-1} \alpha_{*} & \rightarrow \text { id } \\
\text { id } & \rightarrow \alpha_{*} \alpha^{-1}\end{aligned}$ & $\begin{aligned} \underline{\alpha}^{-1} \underline{\alpha}_{*} & \rightarrow \text { id } \\
\text { id } & \rightarrow \underline{\alpha}_{*} \underline{\alpha}^{-1}\end{aligned}$ & $\begin{array}{l}(7.14) \\
(7.15)\end{array}$ & $\begin{aligned} \mathbf{L} \alpha^{-1} \mathbf{R} \alpha_{*} & \rightarrow \text { id } \\
\text { id } & \rightarrow \mathbf{R} \alpha_{*} \mathbf{L} \alpha^{-1}\end{aligned}$ \\
\hline & \multicolumn{4}{|c|}{ Let $A$ be a k-algebra and $Y \stackrel{\alpha}{\rightarrow} X$ a separated, locally proper morphism of top. spaces with $\alpha_{!}: \operatorname{Mod}\left(Y_{A}\right) \rightarrow \operatorname{Mod}\left(X_{A}\right)$ of finite cohomological dimension. } \\
\hline \multirow[t]{2}{*}{ (T2.6) } & $\operatorname{Hom}\left(-, \alpha_{!}^{!}-\right) \stackrel{\sim}{\longrightarrow} \operatorname{Hom}\left(\alpha_{!}-,-\right)$ & $\underline{\operatorname{Hom}}\left(-, \underline{\alpha}^{!}-\right) \stackrel{\sim}{\longrightarrow} \underline{\operatorname{Hom}}\left(\underline{\alpha}_{!}-,-\right)$ & $(7.87)$ & $\mathbf{R H o m}\left(-, \alpha^{!}-\right) \stackrel{\sim}{\longrightarrow} \mathbf{R} \operatorname{Hom}\left(\mathbf{R} \alpha_{!}-,-\right)$ \\
\hline & \multicolumn{4}{|c|}{ Let $A$ be a k-algebra and $\begin{array}{rl}Y^{\prime} & \stackrel{\beta^{\prime}}{\rightarrow} Y \text { a cartesian diagram of top. spaces with } \alpha \text { separated, loc. proper, and } \alpha_{!}: \operatorname{Mod}\left(Y_{A}\right) \rightarrow \operatorname{Mod}\left(X_{A}\right) \text { of finite cohom. dimension } \\
& \downarrow_{\alpha^{\prime}} \downarrow^{\alpha} \\
X^{\prime} \stackrel{\beta}{\rightarrow} X & X\end{array}$} \\
\hline $\begin{array}{l}(\mathrm{T} 2.7) \\
(\mathrm{T} 2.8)\end{array}$ & $\begin{array}{l}\alpha_{1} \beta_{*}^{\prime} \rightarrow \beta_{*} \alpha_{!}^{\prime} \\
\beta_{\beta^{\prime}}^{\prime} \alpha^{\prime \prime} \rightarrow \alpha^{\prime} \beta_{*}^{\prime} \\
\end{array}$ & 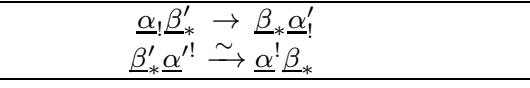 & $\begin{array}{l}(7.76) \\
(7.79)\end{array}$ & $\begin{array}{l}\mathbf{R} \alpha_{!} \mathbf{R} \beta_{*}^{\prime} \rightarrow \mathbf{R} \beta_{*} \mathbf{R} \alpha_{!}^{\prime} \\
\left(\mathbf{R} \beta_{*}^{\prime}\right) \alpha^{\prime !} \stackrel{\sim}{\rightarrow} \alpha^{!} \mathbf{R} \beta_{*}\end{array}$ \\
\hline
\end{tabular}




\subsection{Some commutative diagrams in $\mathrm{ENH}_{\mathrm{k}}$.}

1.7.1. As mentioned before, some of the relations between the six functors are encoded by commutative diagrams. For example, given a morphism $\left(\operatorname{Sh}(\mathcal{Y}), \mathcal{O}_{\mathcal{Y}}\right) \stackrel{\alpha}{\rightarrow}$ $\left(\operatorname{Sh}(\mathcal{X}), \mathcal{O}_{\mathcal{X}}\right)$ of k-ringed topoi, the fact that $\left(\mathbf{L} \alpha^{*}, \mathbf{R} \alpha_{*}\right)$ is a pair of adjoint functors is more precisely encoded by a quadruple $\left(\mathbf{L} \alpha^{*}, \mathbf{R} \alpha_{*}\right.$, id $\stackrel{\eta}{\rightarrow} \mathbf{R} \alpha_{*} \mathbf{L} \alpha^{*}, \mathbf{L} \alpha^{*} \mathbf{R} \alpha_{*} \stackrel{\theta}{\rightarrow}$ id $)$ where $\eta$ and $\theta$ are unit and counit of the adjunction such that the two diagrams
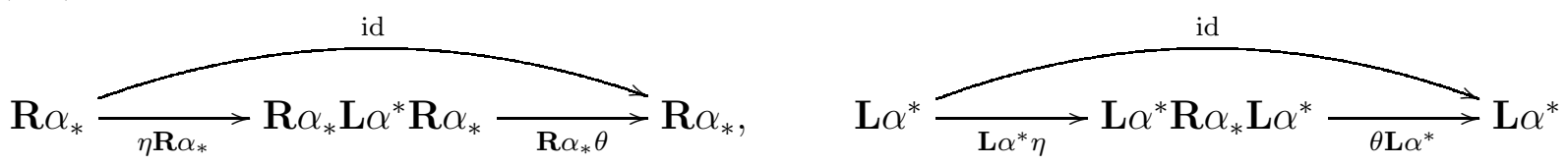

are commutative (these are the triangle identities). The unit and counit 2-morphisms in TRCAT $_{k}$ appear as the rightmost entries of rows (T1.8) and (T1.7) in table 1. They are lifted to the corresponding middle entries id $\stackrel{(\mathrm{T} 1.8)}{\longrightarrow} \underline{\alpha}_{*} \underline{\alpha}^{*}$ and $\underline{\alpha}^{*} \underline{\alpha}_{*} \stackrel{(\mathrm{T} 1.7)}{\longrightarrow}$ id of these rows which are 2-morphisms in $\mathrm{ENH}_{\mathrm{k}}$. Therefore it makes sense to ask whether the datum $\left(\underline{\alpha}^{*}, \underline{\alpha}_{*},(\mathrm{~T} 1.8),(\mathrm{T} 1.7)\right)$ defines an adjunction in $\mathrm{ENH}_{\mathrm{k}}$. This is indeed the case, the two diagrams
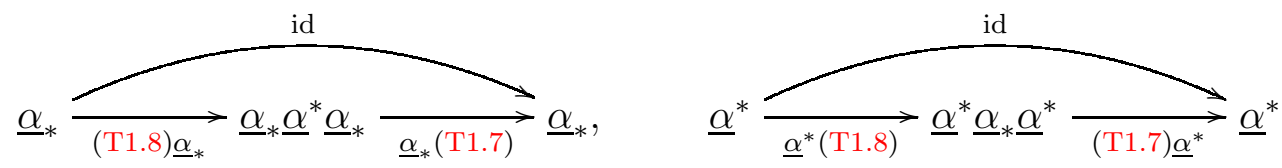

in $\mathrm{ENH}_{\mathrm{k}}$ are commutative (see Proposition 6.27).

1.7.2. In the rest of this section 1.7 we give further similar examples of commutative diagrams in $\mathrm{ENH}_{\mathrm{k}}$ but do not display the corresponding commutative diagrams in $\mathrm{TRCAT}_{\mathrm{k}}$. As above, when referring to a row of table 1, we often just mean the entry in the obvious column.

1.7.3. If $(\mathcal{X}, \mathcal{O})$ is a k-ringed site, $(\underline{\mathbb{I}}(\mathcal{X}), \underline{\mathbb{Q}}, \underline{\mathcal{O}},(\mathrm{T} 1.3),(\mathrm{T} 1.1),(\mathrm{T} 1.2),(\mathrm{T} 1.4))$ is a symmetric monoidal object in $\mathrm{ENH}_{\mathrm{k}}$, see Lemma 6.29. This means that the following diagrams in $\mathrm{ENH}_{\mathrm{k}}$ commute.
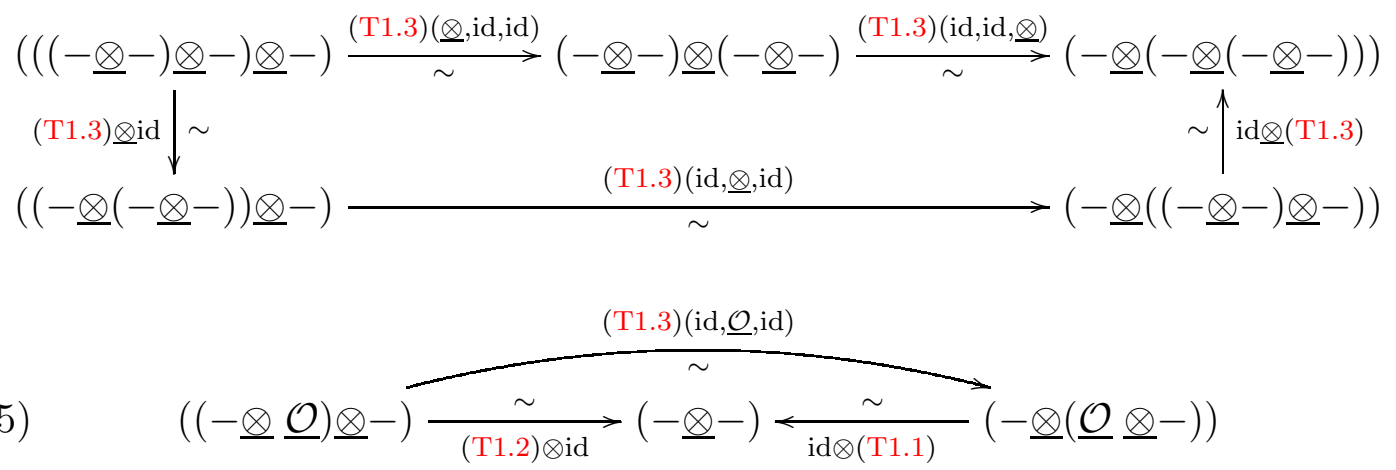


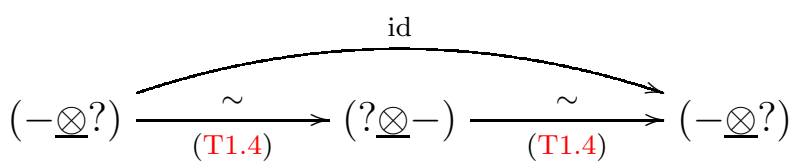

1.7.4. If $\left(\operatorname{Sh}(\mathcal{Z}), \mathcal{O}_{\mathcal{Z}}\right) \stackrel{\beta}{\rightarrow}\left(\operatorname{Sh}(\mathcal{Y}), \mathcal{O}_{\mathcal{Y}}\right) \stackrel{\alpha}{\rightarrow}\left(\operatorname{Sh}(\mathcal{X}), \mathcal{O}_{\mathcal{X}}\right)$ are morphisms of k-ringed topoi, the 2-isomorphisms $\underline{(\alpha \beta)}_{*} \stackrel{(\mathrm{T} 1.12)}{\sim} \underline{\alpha}_{*} \underline{\beta}_{*}$ and $\underline{\beta}^{*} \underline{\alpha}^{*} \stackrel{(\mathrm{T} 1.14)}{\sim} \underline{(\alpha \beta)^{*}}$ are conjugate (with respect to the adjunctions $\left(\underline{(\alpha \beta)^{*}}, \underline{\left.(\alpha \beta)_{*}\right)}\right.$ ) and $\left(\underline{\beta}^{*} \underline{\alpha}^{*}, \underline{\alpha}_{*} \underline{\beta}_{*}\right)$ ), see Proposition 6.28. This means that the following diagram in $\mathrm{ENH}_{\mathrm{k}}$ commutes.

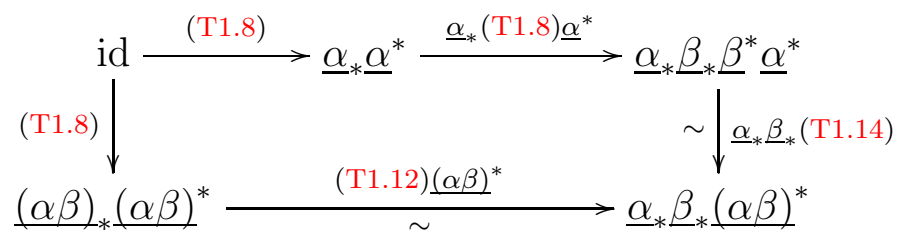

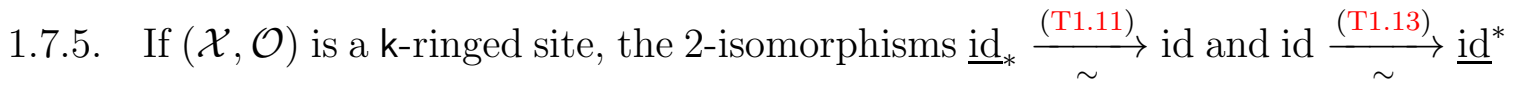
are conjugate (with respect to the adjunctions $\left(\underline{\mathrm{id}}^{*}, \underline{\mathrm{id}}_{*}\right)$ and (id, id)), see Proposition 6.28. This means that the following diagram in $\mathrm{ENH}_{\mathrm{k}}$ commutes.

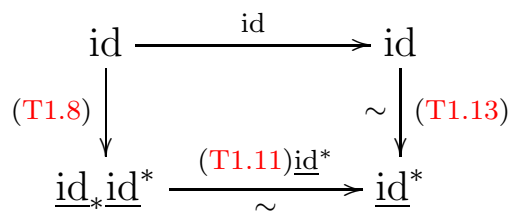

1.7.6. If $A$ is a commutative k-algebra and $\alpha: Y \rightarrow X$ is a separated, locally proper morphism of topological spaces with $\alpha_{!}: \operatorname{Mod}\left(Y_{A}\right) \rightarrow \operatorname{Mod}\left(X_{A}\right)$ of finite cohomological dimension, then the datum $\left(\underline{\alpha}_{!}, \underline{\alpha}^{!},(\mathrm{T} 1.19),(\mathrm{T} 1.18)\right)$ is an adjunction in $\mathrm{ENH}_{\mathrm{k}}$, see Proposition 7.18. This means that the following diagrams in $\mathrm{ENH}_{\mathrm{k}}$ commute.
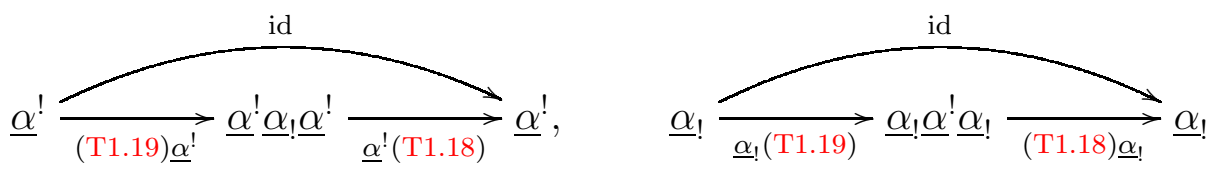

\section{Description of the MAin RESUlts}

Our aim in 2.1 is to enable the reader to use the dg k-enhanced six functor formalism without having to digest all technical details of this long article. In 2.2 we explain how certain isomorphisms lift from the triangulated level to the dg level. The ingredients from enriched model category theory we use are then explained in 2.3. Remember that $k$ always denotes a field.

\subsection{User's guide to the dg k-enhanced six functor formalism.}


2.1.1. Dg k-enhancements considered - lifts of derived categories of sheaves. Let $X$ be a k-ringed space. Let $\underline{\mathrm{C}}(X)$ be the dg k-category of complexes of sheaves on $X$ and $\mathbb{I}(X)$ its full dg k-subcategory of h-injective complexes of injective sheaves. Then $\underline{I}(X)$ is a strongly pretriangulated dg k-category. Its homotopy category $[\mathbb{I}(X)]$ is a triangulated k-category and the obvious functor is an equivalence $[\mathbb{I}(X)] \stackrel{\sim}{\rightarrow} \mathrm{D}(X)$ of triangulated $\mathrm{k}$-categories. This means that $\mathbb{I}(X)$ is a dg k-enhancement of $\mathrm{D}(X)$. In 2.3.2 and 2.3.3 we construct an equivalence

$$
\overline{[\mathbf{i}]}: \mathrm{D}(X) \stackrel{\sim}{\rightarrow}[\underline{\mathbb{I}}(X)]
$$

of triangulated k-categories in the other direction. Fixing this equivalence allows a very precise formulation of our results.

\subsubsection{Lifts of the six functors. We provide dg k-functors}

$$
\begin{aligned}
(-) \underline{\otimes}(-): \underline{\mathbb{I}}(X) \otimes \underline{\mathbb{I}}(X) & \rightarrow \underline{\mathbb{I}}(X), & \underline{\mathcal{H} o m}(-,-): \underline{\mathbb{I}}(X)^{\mathrm{op}} \otimes \underline{I}(X) & \rightarrow \underline{\mathbb{I}}(X), \\
\underline{\alpha}^{*}: \underline{\mathbb{I}}(X) & \rightarrow \underline{\mathbb{I}}(Y), & \underline{\alpha}_{*}: \underline{\mathbb{I}}(Y) & \rightarrow \underline{\mathbb{I}}(X), \\
\underline{\alpha}_{!}: \underline{\mathbb{I}}(Y) & \rightarrow \underline{\mathbb{I}}(X), & \underline{\alpha}^{!}: \underline{\mathbb{I}}(X) & \rightarrow \underline{\mathbb{I}}(Y)
\end{aligned}
$$

where $\alpha: Y \rightarrow X$ is a morphism of $\mathrm{k}$-ringed spaces which is assumed to satisfy some additional properties whenever !-functors are involved, cf. 2.1.7. The construction of these $\mathrm{dg}$ k-functors is explained in 2.3.2 and 2.3.4. They lift the triangulated kfunctors $\otimes^{\mathbf{L}}$, RHom, $\mathbf{L} \alpha^{*}, \mathbf{R} \alpha_{*}, \mathbf{R} \alpha_{!}, \alpha^{!}$in the following sense. The functors $\underline{\alpha}^{*}$ and $\underline{\alpha}_{*}$ induce, by passing to homotopy categories, functors $\left[\underline{\alpha}^{*}\right]:[\underline{\mathbb{I}}(X)] \rightarrow[\underline{\mathbb{I}}(Y)]$ and $\left[\underline{\alpha}_{*}\right]:[\mathbb{I}(Y)] \rightarrow[\mathbb{I}(X)]$ of triangulated k-categories, and the diagrams
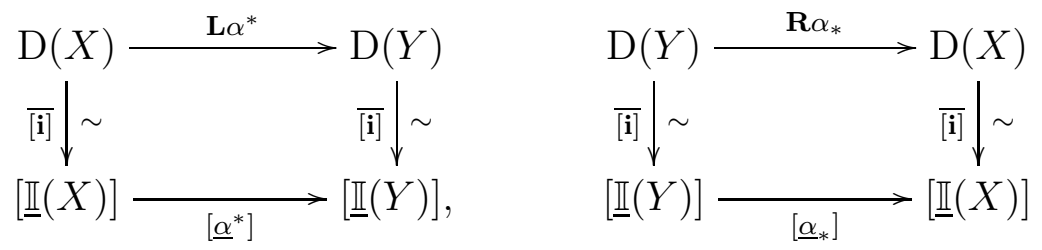

in TRCAT $_{\mathrm{k}}$ commute up to canonical 2-isomorphisms; there are similar canonically 2-commutative diagrams relating the dg k-functors $\underline{\otimes}, \underline{\mathcal{H} o m}, \underline{\alpha}, \underline{\alpha}^{!}$to the functors $\otimes^{\mathbf{L}}, \mathbf{R} \mathcal{H}$ om, $\mathbf{R} \alpha_{!}, \alpha^{!}$, see $6.3,7.4$.

2.1.3. The 2-multicategory $\mathrm{ENH}_{\mathrm{k}}$ of enhancements. In order to lift the relations between the six functors we introduce the k-linear 2-multicategory $\mathrm{ENH}_{\mathrm{k}}$ of enhancements (see 5.2). Its objects are additive pretriangulated dg k-categories. The objects relevant here are the dg k-categories $\mathbb{I}(X)$ introduced above. To define the morphism k-categories of $\mathrm{ENH}_{\mathrm{k}}$ we need a definition. 
Given objects $\underline{\mathbb{I}}(X)$ and $\underline{\mathbb{I}}(Y)$ let $\tau: F^{\prime} \rightarrow F$ be a morphism in $\operatorname{DGCAT}_{\mathrm{k}}(\underline{\mathbb{I}}(X) ; \underline{\mathbb{I}}(Y))$ as illustrated by the picture

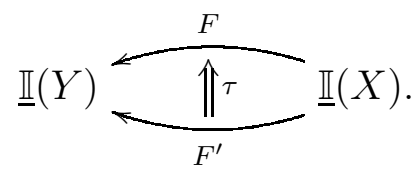

We say that $\tau$ is an objectwise homotopy equivalence if $\tau_{I}: F^{\prime}(I) \rightarrow F(I)$ is a homotopy equivalence (or, equivalently, a quasi-isomorphism) for all $I \in \mathbb{I}(X)$. An equivalent condition is that the induced morphism $[\tau]:\left[F^{\prime}\right] \rightarrow[F]$ in $\operatorname{TRCAT}_{\mathbf{k}}([\underline{\mathbb{I}}(X)] ;[\underline{\mathbb{I}}(Y)])$ is an isomorphism.

We define the morphism k-category $\mathrm{ENH}_{\mathrm{k}}(\mathbb{I}(X) ; \underline{\mathbb{I}}(Y))$ as the target of the additive k-localization (see Definition 5.4) of $\operatorname{DGCAT}_{\mathrm{k}}(\underline{\mathbb{I}}(X) ; \underline{\mathbb{I}}(Y)$ ) with respect to the set of objectwise homotopy equivalences; a similar definition is used when several source objects are involved. Note that the set of 1-morphisms in $\mathrm{ENH}_{\mathrm{k}}$ with fixed sources and target coincides with the corresponding set of 1-morphisms in DGCAT $_{k}$. The six dg k-functors $\underline{\otimes}, \underline{\mathcal{H} o m}, \underline{\alpha}^{*}, \underline{\alpha}_{*}, \underline{\alpha}_{!}, \underline{\alpha}^{!}$are 1-morphisms in $\mathrm{ENH}_{\mathrm{k}}$.

Clearly, taking homotopy categories defines a functor $[-]: \mathrm{ENH}_{\mathrm{k}} \rightarrow \mathrm{TRCAT}_{\mathrm{k}}$. This is the functor (1.1) mentioned above.

2.1.4. Lifts of relations. Explicit zig-zags of $\mathrm{dg}$ k-natural transformations define a 2-morphism id $\rightarrow \underline{\alpha}_{*} \underline{\alpha}^{*}$ and a 2-isomorphism $\underline{\alpha}_{*} \underline{\mathcal{H} o m}\left(-, \underline{\alpha}^{!}(-)\right) \stackrel{\sim}{\rightarrow} \underline{\mathcal{H} o m}\left(\underline{\alpha}_{!}(-),-\right)$ in $\mathrm{ENH}_{\mathrm{k}}$ whose images under (1.1) coincide modulo the equivalences (2.1) and the canonical 2-isomorphisms (cf. (2.3)) with the 2-morphism id $\rightarrow \mathbf{R} \alpha_{*} \mathbf{L} \alpha^{*}$ and the Verdier duality 2-isomorphism $\mathbf{R} \alpha_{*} \mathbf{R} \mathcal{H} o m\left(-, \alpha^{!}(-)\right) \stackrel{\sim}{\rightarrow} \mathbf{R} \mathcal{H o m}\left(\mathbf{R} \alpha_{!}(-),-\right)$, respectively. Similarly, we define all the 2-(iso)morphisms in the middle column of table 1 on page 6 and show that they lift the corresponding 2-(iso)morphisms in the right column. Combining these 2-(iso)morphisms provides many other lifts. We have assembled some of them in table 2 on page 7 . The equation numbers in the middle columns of these tables refer to the corresponding 2-(iso)morphisms in the main body of this article. See 2.1.7 for more explanations concerning these tables.

Moreover, we establish that $\left(\underline{\alpha}^{*}, \underline{\alpha}_{*}\right)$ together with the 2-morphisms id $\rightarrow \underline{\alpha}_{*} \underline{\alpha}^{*}$ and $\underline{\alpha}^{*} \underline{\alpha}_{*} \rightarrow$ id (in rows (T1.8) and (T1.7)) form an adjunction in $\mathrm{ENH}_{\mathrm{k}}$. We also show that $(\underline{\mathbb{I}}(X), \underline{\otimes})$ together with $\underline{\mathcal{O}}$ and the middle entries of rows (T1.3), (T1.1), (T1.2), (T1.4) is a symmetric monoidal object in $\mathrm{ENH}_{\mathrm{k}}$. These two facts are encoded in commutativity of the five diagrams in $(1.3),(1.4),(1.5),(1.6)$ in 1.7. We also prove commutativity of the other diagrams in (1.7), (1.8), (1.9) there. They lift standard relations between some of the six functors. There are many other commutative diagrams relating the six functors. We strongly believe that their counterparts in $\mathrm{ENH}_{\mathrm{k}}$ can be shown to commute using our techniques. We have restricted ourselves to the list in 1.7 for reasons of space and time. 
2.1.5. 2-Morphisms in $\mathrm{ENH}_{\mathrm{k}}$. All 2-morphisms in $\mathrm{ENH}_{\mathrm{k}}$ can be represented by zigzags of 2-morphisms in DGCAT $_{k}$ where the arrows pointing in the wrong direction are objectwise homotopy equivalences. As already mentioned, all 2-morphisms in the middle columns of tables 1 and 2 are explicitly defined in this way; the 2-morphisms marked as 2-isomorphisms there are defined by zig-zags of objectwise homotopy equivalences, with one exception, namely the 2-isomorphism $\underline{\beta}_{*}^{\prime} \underline{\alpha}^{\prime} \stackrel{\sim}{\rightarrow} \underline{\alpha}^{!} \underline{\beta}_{*}$ in (T2.8).

In general, we do not know whether any invertible 2-morphism in $\mathrm{ENH}_{\mathrm{k}}$ can be represented by a zig-zag (or even a roof) of objectwise homotopy equivalences. We also would like to know whether the functor [-]: $\mathrm{ENH}_{\mathrm{k}} \rightarrow \mathrm{TRCAT}_{\mathrm{k}}$ reflects 2isomorphisms since an affirmative answer to this question would give a useful method to show that certain 2-morphisms in $\mathrm{ENH}_{\mathrm{k}}$ are invertible. These problems seem to be of set-theoretical origin and have partial solutions explained in 9; a more precise discussion of properties we would like to have is also given there.

Therefore, it might be useful to remember the definition of certain 2-(iso)morphisms in $\mathrm{ENH}_{\mathrm{k}}$ in applications. Nevertheless, the definition of the 2-category $\mathrm{ENH}_{\mathrm{k}}$ seems very natural to us; for example, it would be very annoying to formulate the neat statement that $\left(\underline{\alpha}^{*}, \underline{\alpha}_{*}\right)$ is an adjunction in $\mathrm{ENH}_{\mathrm{k}}$ in terms of zig-zags: this would amount to the big commutative diagram (6.82) (plus the argument that blue and green arrow there coincide plus the same amount of work for the other triangle identity).

2.1.6. k-ringed topoi. More generally, all our constructions involving the four functors $\otimes^{\mathbf{L}}, \mathbf{R} \mathcal{H}$ om, $\mathbf{L} \alpha^{*}, \mathbf{R} \alpha_{*}$ work for k-ringed sites $\left(X, \mathcal{O}_{X}\right)$ and for morphisms $\alpha:\left(\operatorname{Sh}(Y), \mathcal{O}_{Y}\right) \rightarrow\left(\operatorname{Sh}(X), \mathcal{O}_{X}\right)$ of k-ringed topoi. In the rest of this section we use this language and extend our notation in the obvious way from k-ringed spaces to k-ringed sites.

2.1.7. Some explanations concerning the tables and !-functors. The appearance of kringed sites and morphisms of k-ringed topoi in tables 1 and 2 is justified by 2.1.6. All k-algebras in these tables are assumed to be commutative.

The symbol $\underline{\mathcal{O}}$ is an h-injective complex of injective sheaves lifting the structure sheaf $\mathcal{O}$ (see 2.3.4 for the definition, and also Remark 5.8). The 1-morphisms $\underline{\Gamma}$ and

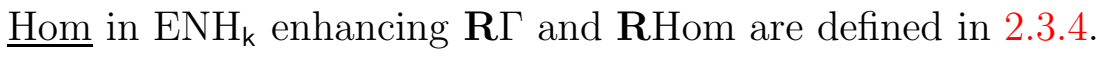

We also need to explain the symbol $\underline{\alpha}^{-1}$. Whenever a commutative k-algebra $A$ and a morphism $\alpha: Y \rightarrow X$ of topological spaces are given we view $\alpha$ as a morphism $X_{A} \rightarrow Y_{A}$ of k-ringed spaces in the obvious way where $X_{A}$ and $Y_{A}$ denote $X$ and $Y$ equipped with the constant sheaf of k-algebras with stalk $A$. Then $\alpha^{*}=\alpha^{-1}$ is exact (and $\alpha$ is flat). In this case we define a 1-morphism $\underline{\alpha}^{-1}$ (see 2.3.4) lifting $\mathbf{L} \alpha^{-1}$ and a 2-isomorphism $\underline{\alpha}^{*} \stackrel{\sim}{\rightarrow} \underline{\alpha}^{-1}$ lifting $\mathbf{L} \alpha^{*} \stackrel{\sim}{\rightarrow} \mathbf{L} \alpha^{-1}$ (see row (T1.15)).

When working with !-functors we follow the approach of [SS16] to impose conditions on maps rather than spaces (which are traditionally assumed to be locally compact), cf. 7.1. We then only consider morphisms of k-ringed spaces coming from a morphism 
of topological spaces and a k-algebra as explained above; the only reason for this restriction is that a more general theory is not yet documented in the literature.

2.1.8. The 2-multicategory of formulas. The k-linear 2-multicategory $\mathrm{FML}_{\mathrm{k}}$ of formulas is our formal tool to describe all relations between functors between derived categories of sheaves that we can lift to dg k-enhancements. We explain how to summarize our lifting results using this multicategory.

Essentially, $\mathrm{FML}_{\mathrm{k}}$ is the free k-linear 2-multicategory with relations whose objects are symbols $X$ (and $X^{\text {op }}$ ), for each k-ringed site $X$, whose generating 1-morphisms

are symbols $\otimes, \mathcal{H}$ Hom, $\alpha^{*}, \alpha_{*}, \alpha_{!}, \alpha^{!}$(and $\mathcal{O}, \alpha^{-1}, \Gamma$, Hom) and whose generating 2-morphisms are the "formulas" given by the entries of the left column of table 1; the relations we impose just say that all the generating 2 -morphisms labeled $\sim$ are invertible. We refer the reader to 8 for a precise definition of $\mathrm{FML}_{\mathrm{k}}$ (there are a few more generating 2-morphisms and relations that are not important and ignored here).

There is an obvious interpretation functor

$$
\mathrm{FML}_{\mathrm{k}} \rightarrow \mathrm{TRCAT}_{\mathrm{k}}
$$

of k-linear 2-multicategories mapping $X$ to $\mathrm{D}(X), X^{\text {op }}$ to $\mathrm{D}(X)^{\mathrm{op}}$, mapping the six generating 1-morphisms to the six functors $\otimes^{\mathbf{L}}, \mathbf{R} \mathcal{H} o m, \mathbf{L} \alpha^{*}, \mathbf{R} \alpha_{*}, \mathbf{R} \alpha_{!}, \alpha^{\text {! }}$, and mapping the generating 2-morphisms to the corresponding 2-morphisms in the right column in table 1.

Theorem 2.1 (cf. Theorems 8.3, 8.5, Remark 8.4). There is an interpretation functor

$$
\mathrm{FML}_{\mathrm{k}} \rightarrow \mathrm{ENH}_{\mathrm{k}}
$$

of k-linear 2-multicategories mapping $X$ to $\mathbb{I}(X)$, mapping the six generating 1 morphisms to the six dg $\mathrm{k}$-functors $\underline{\otimes}, \underline{\mathcal{H} o m}, \underline{\alpha}^{*}, \underline{\alpha}_{*}, \underline{\alpha}_{!}, \underline{\alpha}^{!}$, and mapping the generating 2-morphisms to the corresponding 2-morphisms in the middle column of table 1. If we view all diagrams in 1.7 (except diagram (1.2)) as diagrams in $\mathrm{FML}_{\mathrm{k}}$ by replacing underlines by underdots, the interpretation functor (2.6) maps these diagrams to commutative diagrams (namely to the commutative diagrams in 1.7), and similarly for the commutative diagrams in Lemmas 6.32 and 7.20. Moreover, there is a pseudo-natural transformation $\omega$ as illustrated by the diagram

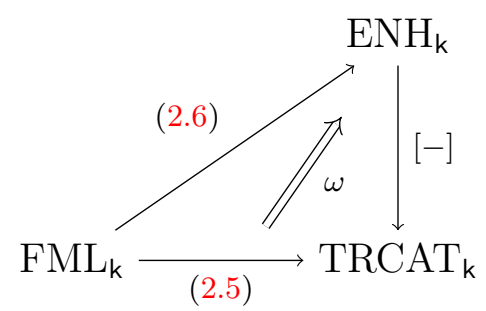


that maps an object $X$ to the 1-morphism (2.1) (which is an equivalence of triangulated k-categories) and that maps the generating 1-morphisms $\alpha^{*}, \alpha_{*}$ to the canonical 2isomorphisms making the diagrams in (2.3) commutative, respectively, and similarly for the other generating 1-morphisms.

Theorem 2.1 follows immediately from our lifting results explained in 2.1.1, 2.1.2, 2.1.4.

For the sake of completeness let us mention that the entries of the left column in table 2 are defined in the obvious way as compositions of suitable generating 2morphisms such that they are mapped to the entries in the other two columns under our interpretation functors.

2.1.9. The meaning of Theorem 1.1. The precise meaning of Theorem 1.1 consists of

(a) the definition of the k-linear 2-multicategory $\mathrm{ENH}_{\mathrm{k}}$;

(b) the definition of the 1-morphisms $\underline{\otimes}, \underline{\mathcal{H} o m}, \underline{\alpha}^{*}, \underline{\alpha}_{*}, \underline{\alpha}_{!}, \underline{\alpha}^{!}$in $\mathrm{ENH}_{\mathrm{k}}$;

(c) the definition of all the 2-(iso)morphisms in $\mathrm{ENH}_{\mathrm{k}}$ in the middle column of table 1 by explicit zig-zags;

(d) the definition of the k-linear 2-multicategory $\mathrm{FML}_{\mathrm{k}}$;

(e) the statement of Theorem 2.1 saying in a very precise way that the previous data lift the part of Grothendieck-Verdier-Spaltenstein's six functor formalism considered in this article to the k-linear 2-multicategory $\mathrm{ENH}_{\mathrm{k}}$.

\subsection{Lifting actions and (iso)morphisms from the derived level to the $\mathbf{d g}$}

level. It may be helpful to look at the example in 2.2.3 first.

2.2.1. Lifting actions. Let

$$
M:\left(\mathrm{D}\left(X_{1}\right)^{\varepsilon_{1}}, \ldots, \mathrm{D}\left(X_{n}\right)^{\varepsilon_{n}}\right) \rightarrow \mathrm{D}(Y)
$$

be a 1-morphism in TRCAT $_{\mathrm{k}}$ where $X_{1}, \ldots, X_{n}, Y$ are k-ringed sites and $\varepsilon_{i} \in\{\emptyset$, op $\}$. Then $M$ gives rise to a k-functor

$$
M: \mathrm{D}\left(X_{1}\right)^{\varepsilon_{1}} \otimes \cdots \otimes \mathrm{D}\left(X_{n}\right)^{\varepsilon_{n}} \rightarrow \mathrm{D}(Y)
$$

i. e. a "left $\mathrm{D}\left(X_{1}\right)^{\varepsilon_{1}} \otimes \cdots \otimes \mathrm{D}\left(X_{n}\right)^{\varepsilon_{n}}$-module with values in $\mathrm{D}(Y)$ ". If $M$ is a composition of 1-morphisms which can be lifted to $\mathrm{ENH}_{\mathrm{k}}$, i. e. if $M$ is in the image of the interpretation functor (2.5), there is a dg k-functor

$$
\underline{M}: \underline{\mathbb{I}}\left(X_{1}\right)^{\varepsilon_{1}} \otimes \cdots \otimes \underline{\mathbb{I}}\left(X_{n}\right)^{\varepsilon_{n}} \rightarrow \underline{\mathbb{I}}(Y),
$$

i. e. a "left $\operatorname{dg} \mathbb{I}\left(X_{1}\right)^{\varepsilon_{1}} \otimes \cdots \otimes \underline{I}\left(X_{n}\right)^{\varepsilon_{n}}$-module with values in $\underline{\mathbb{I}}(Y)$ ", such that

$$
[\underline{M}]:\left[\mathbb{I}\left(X_{1}\right)^{\varepsilon_{1}}\right] \otimes \cdots \otimes\left[\underline{\mathbb{I}}\left(X_{n}\right)^{\varepsilon_{n}}\right] \rightarrow[\mathbb{I}(Y)]
$$

is canonically identified with (2.9). In the special case that $Y=(\mathrm{pt}, \mathrm{k})$ we have $\underline{\mathbb{I}}(Y)=\underline{\mathrm{C}}(\mathrm{k})$ and $\underline{M}$ is a left $\mathrm{dg} \underline{\mathbb{I}}\left(X_{1}\right)^{\varepsilon_{1}} \otimes \cdots \otimes \underline{\mathbb{I}}\left(X_{n}\right)^{\varepsilon_{n}}$-module. 
2.2.2. Lifting (iso)morphisms. Let $\rho: M_{1} \rightarrow M_{2}$ be a 2-morphism in $\mathrm{TRCAT}_{\mathrm{k}}$ where $M_{1}$ and $M_{2}$ are as in (2.8). In particular, we can view $\rho$ as a morphism of left $\mathrm{D}\left(X_{1}\right)^{\varepsilon_{1}} \otimes \cdots \otimes \mathrm{D}\left(X_{n}\right)^{\varepsilon_{n}}$-module with values in $\mathrm{D}(Y)$. If $\rho$ and $M_{1}$ and $M_{2}$ are in the image of the interpretation functor (2.5), there is a 2-morphism $\varrho: \underline{M}_{1} \rightarrow \underline{M}_{2}$ in $\mathrm{ENH}_{\mathrm{k}}$ such that $[\varrho]:\left[\underline{M}_{1}\right] \rightarrow\left[\underline{M}_{2}\right]$ is canonically identified with $\rho$.

If we know that $\varrho$ can be represented by a zig-zag of objectwise homotopy equivalences, then $\underline{M}_{1}$ and $\underline{M}_{2}$ are isomorphic in the "derived category of left $\operatorname{dg} \mathbb{I}\left(X_{1}\right)^{\varepsilon_{1}} \otimes$ $\cdots \otimes \mathbb{I}\left(X_{n}\right)^{\varepsilon_{n}}$-modules with values in $\mathbb{I}(Y)$ ". In the special case that $Y=(\mathrm{pt}, \mathrm{k})$ they are isomorphic in the more familiar derived category of left $\operatorname{dg} \mathbb{I}\left(X_{1}\right)^{\varepsilon_{1}} \otimes \cdots \otimes \mathbb{I}\left(X_{n}\right)^{\varepsilon_{n}}$ modules.

2.2.3. An example. Let $\alpha \circ \beta^{\prime}=\beta \circ \alpha^{\prime}$ be a cartesian diagram as in the row above (T1.27) in table 1 on page 6 ; assume that $\beta$ is separated and locally proper with $\beta_{!}: \operatorname{Mod}\left(X_{A}^{\prime}\right) \rightarrow \operatorname{Mod}\left(X_{A}\right)$ of finite cohomological dimension. Then there is a 2isomorphism

$$
\mathbf{R} \operatorname{Hom}\left(\mathbf{R} \alpha_{!}(-), \mathbf{R} \mathcal{H o m}\left(\mathbf{R} \beta_{!}(-),-\right)\right), \cong \mathbf{R} \operatorname{Hom}\left(\mathbf{R} \alpha_{!}^{\prime} \mathbf{L} \beta^{\prime *}(-) \otimes^{\mathbf{L}}(-), \beta^{!}(-)\right)
$$

between triangulated $\mathrm{k}$-trilinear functors from $\mathrm{D}(Y)^{\mathrm{op}} \times \mathrm{D}\left(X^{\prime}\right)^{\mathrm{op}} \times \mathrm{D}(X)$ to $\mathrm{D}(\mathrm{pt})$, by (T1.21), (T2.2), (T1.15), (T1.27), (T2.1). The above discussion shows that it lifts to a 2-isomorphism

$$
\underline{\operatorname{Hom}}\left(\underline{\alpha}_{!}(-), \underline{\mathcal{H} o m}\left(\underline{\beta}_{!}(-),-\right)\right) \cong \underline{\operatorname{Hom}}\left(\underline{\alpha}_{!}^{\prime} \underline{\beta}^{\prime *}(-) \underline{\mathbb{Q}}(-), \underline{\beta}^{!}(-)\right)
$$

in $\mathrm{ENH}_{\mathrm{k}}$ which can be represented by a zig-zag of objectwise homotopy equivalences; hence it gives rise to an isomorphism in the derived category of left $\operatorname{dg} \mathbb{I}(Y)^{\mathrm{op}} \otimes$ $\underline{\mathbb{I}}\left(X^{\prime}\right)^{\mathrm{op}} \otimes \underline{\mathbb{I}}(X)$-modules.

2.3. Model categories and dg k-categories. The key ingredients for our results come from the interplay between model category theory and enriched category theory. Recall that a dg k-category is a category enriched in the monoidal category of complexes of k-vector spaces. We usually denote a dg k-category by an underlined symbol and omit the underline when referring to the underlying category.

2.3.1. Dg $\mathrm{k}$-enriched functorial factorizations. Any model category $\mathcal{M}$ admits functorial factorizations and in particular functorial cofibrant and fibrant resolutions. If $\mathcal{N}$ is the underlying category of a dg k-category $\underline{\mathcal{N}}$ it is natural to ask whether it admits $\mathrm{dg}$ k-enriched functorial factorizations. We provide some general criteria to ensure a positive answer to this question in Theorem 3.6. This result is the adaptation to dg k-categories of general results from enriched model category theory [Shu09, Rie14]. As a consequence we obtain:

Theorem 2.2 (see Theorems 4.17, 4.20, 4.24). Let $\mathrm{k}$ be a field. Consider the following list of $d g \mathrm{k}$-categories and model structures on their underlying categories turning them into model categories. 
(a) $\underline{\mathrm{C}}(X)$ with $\mathrm{C}(X)$ carrying the injective model structure (see 4.1.1) or the extension-by-zero model structure (see 4.2.2), for a $\mathrm{k}$-ringed site $(X, \mathcal{O})$;

(b) the dg k-category $\underline{\mathbb{M o d}}(\underline{\mathrm{e}})$ of (right) dg modules over a dg k-category $\underline{\mathrm{e}}$, with $\operatorname{Mod}(\underline{\mathrm{e}})$ carrying the injective or the projective model structure (see 4.3.1);

(c) $\underline{\mathrm{C}}(X)$ with $\mathrm{C}(X)$ carrying the flat model structure (see 4.4.1) or the injective model structure, for a $\mathrm{k}$-ringed space $(X, \mathcal{O})$;

Then all the model structures in this list admit $d g \mathrm{k}$-enriched functorial factorizations.

2.3.2. Dg k-enriched injective and flat resolutions. Given a k-ringed site $(X, \mathcal{O})$, the fibrant objects of the injective model structure on $\mathrm{C}(X)$ are precisely the objects of $\mathbb{I}(X)$ and the cofibrant objects of the extension-by-zero model structure on $\mathrm{C}(X)$ are h-flat and have flat components (see Lemma 4.11). Therefore Theorem 2.2.(a) yields the following key ingredient to lift the six functors to dg k-functors.

Corollary 2.3. Let $\mathrm{k}$ be a field. Let $(X, \mathcal{O})$ be a $\mathrm{k}$-ringed site and let $\underline{\mathrm{C}}(X)_{\mathrm{hflat}, \mathrm{cwflat}}$ denote the full $d g \mathrm{k}$-subcategory of $\underline{\mathrm{C}}(X)$ of h-flat and componentwise flat objects. Then there are $d g \mathrm{k}$-functors

$$
\begin{aligned}
& \text { i: } \underline{\mathrm{C}}(X) \rightarrow \underline{\mathbb{I}}(X), \\
& \text { e: } \underline{\mathrm{C}}(X) \rightarrow \underline{\mathrm{C}}(X)_{\text {hflat,cwflat }}
\end{aligned}
$$

together with dg k-natural transformations

$$
\begin{aligned}
\iota: \mathrm{id} \rightarrow \mathbf{i}: \underline{\mathrm{C}}(X) \rightarrow \underline{\mathrm{C}}(X), \\
\epsilon: \mathbf{e} \rightarrow \mathrm{id}: \underline{\mathrm{C}}(X) \rightarrow \underline{\mathrm{C}}(X)
\end{aligned}
$$

whose evaluations $\mathfrak{\iota}_{G}: G \rightarrow \mathbf{i} G$ and $\epsilon_{G}: \mathbf{e} G \rightarrow G$ at each object $G \in \underline{\mathrm{C}}(X)$ are quasiisomorphisms.

The assumption that $\mathrm{k}$ is a field is essential for this key ingredient. We give a counterexample in Lemma 4.4. We fix such "injective" and "flat" resolution functors for each k-ringed site.

2.3.3. Definition of the equivalence (2.1). The dg k-functor i induces a functor

$$
[\mathbf{i}]:[\underline{\mathrm{C}}(X)] \rightarrow[\underline{\mathbb{I}}(X)]
$$

of triangulated k-categories which maps acyclic objects to zero. It factors to an equivalence $\overline{[\mathbf{i}]}: \mathrm{D}(X) \rightarrow[\underline{\mathbb{I}}(X)]$ of triangulated k-categories. This defines the equivalence (2.1). 
2.3.4. Definition of the lifts of the six functors. The first five of the dg k-functors in (2.2) are defined by

$$
\begin{array}{rlrl}
(-) \underline{\otimes}(-) & :=\mathbf{i}(\mathbf{e}(-) \otimes \mathbf{e}(-)), & \underline{\mathcal{H} o m}(-,-) & :=\mathbf{i} \mathcal{H o m}(-,-), \\
\underline{\alpha}^{*} & :=\mathbf{i} \alpha^{*} \mathbf{e}, & \underline{\alpha}_{*}:=\mathbf{i} \alpha_{*}, \\
\underline{\alpha}_{!} & :=\mathbf{i} \alpha ! . & &
\end{array}
$$

The definition of the sixth dg k-functor $\underline{\alpha}^{!}$uses the explicit description of a right adjoint functor $\alpha^{!}$to $\mathbf{R} \alpha_{\text {! }}$. We refer the reader to 7.4. The object $\underline{\mathcal{O}}$ appearing in table 1 is defined by $\underline{\mathcal{O}}:=\mathbf{i} \mathcal{O}$. Further definitions are $\underline{\alpha}^{-1}:=\mathbf{i} \alpha^{-1}, \underline{\Gamma}:=\underline{\sigma}_{*}$ $\left(\right.$ where $\sigma:\left(\operatorname{Sh}(\mathcal{X}), \mathcal{O}_{\mathcal{X}}\right) \rightarrow(\operatorname{Sh}(\mathrm{pt}), \mathrm{k})$ is the structure morphism, see (4.10)), and $\underline{\text { Hom }}:=\mathbf{i} \Gamma \mathcal{H}$ om.

\section{DG k-ENRICHED FUnCTORIAL FACTORIZATIONS}

We provide criteria to ensure that a model structure on a dg k-category admits $\mathrm{dg}$ k-enriched functorial factorizations, see Theorem 3.6. We use the language of enriched categories, in particular of dg categories, and of model categories. All basic facts we need can be found in [Kel05, Kel06, Hir03, Hov99, MP12, Rie14]. We fix a Grothendieck universe $\mathcal{U}$.

3.1. Categories of complexes. Recall that $\mathrm{R}$ is a commutative ring. We say $\mathrm{R}-$ category instead of R-linear category. When size issues seem important we will be more precise and speak for example about R-categories with $\mathcal{U}$-small Hom-sets.

Let $\mathcal{A}$ be an additive R-category. We write $\mathcal{A}^{\mathbb{Z}}$ for the additive R-category of $\mathbb{Z}$-graded objects in $\mathcal{A}$. The additive R-category of (cochain) complexes in $\mathcal{A}$ with cochain maps as morphisms is denoted $\mathrm{C}(\mathcal{A})$. We sometimes view $\mathcal{A}^{\mathbb{Z}}$ as the full subcategory of $\mathrm{C}(\mathcal{A})$ consisting of complexes with vanishing differential. We also have the R-functor $\mathrm{C}(\mathcal{A}) \rightarrow \mathcal{A}^{\mathbb{Z}}$ that forgets the differentials. The two categories $\mathcal{A}^{\mathbb{Z}}$ and $\mathrm{C}(\mathcal{A})$ are abelian if $\mathcal{A}$ is abelian. They come endowed with shift functors $[n]$, for $n \in \mathbb{Z}$. If $f: M \rightarrow N$ is a morphism in $\mathrm{C}(\mathcal{A})$, we denote its cone by Cone $(f)$, i. e. Cone $(f)=N \oplus[1] M$ with differential $d_{\operatorname{Cone}(f)}=\left(\begin{array}{cc}d_{N} & {[1] f} \\ 0 & d_{[1] M}\end{array}\right)$. As usual, we view $\mathcal{A}$ as a full subcategory of $\mathcal{A}^{\mathbb{Z}}$ and $\mathrm{C}(\mathcal{A})$. In particular, if $M$ is an object of $\mathcal{A}$, the shift $[n] M$ for $n \in \mathbb{Z}$ is the complex in $\mathcal{A}$ concentrated in degree $-n$ with $([n] M)^{-n}=M$. There is a canonical monomorphism $[n] M \rightarrow \operatorname{Cone}\left(\operatorname{id}_{[n] M}\right)$ in $\mathrm{C}(\mathcal{A})$ which splits in $\mathcal{A}^{\mathbb{Z}}$.

We write $\underline{\mathrm{C}}(\mathcal{A})$ for the dg R-category of complexes in $\mathcal{A}$. Given complexes $M, N$ in $\mathcal{A}$ we usually use the notation

$$
\begin{aligned}
& \mathrm{C}_{\mathcal{A}}(M, N)=\operatorname{Hom}_{\mathrm{C}(\mathcal{A})}(M, N), \\
& \underline{\mathrm{C}}_{\mathcal{A}}(M, N)=\operatorname{Hom}_{\underline{\mathrm{C}}(\mathcal{A})}(M, N) .
\end{aligned}
$$


The R-module $\mathrm{C}_{\mathcal{A}}(M, N)$ consists of the closed degree zero morphisms in the $\mathrm{dg}$ R-module $\underline{\mathrm{C}}_{\mathcal{A}}(M, N)$, i. e. $\mathrm{C}_{\mathcal{A}}(M, N)=Z^{0}\left(\underline{\mathrm{C}}_{\mathcal{A}}(M, N)\right)$.

As usual, we write $[\underline{\mathrm{C}}(\mathcal{A})]$ for the homotopy R-category of complexes in $\mathcal{A}$. Its objects are complexes in $\mathcal{A}$, and given two such complexes $M, N$ we have

$$
\left[\underline{\mathrm{C}}_{\mathcal{A}}\right](M, N):=\operatorname{Hom}_{[\underline{\mathrm{C}}(\mathcal{A})]}(M, N):=H^{0}\left(\underline{\mathrm{C}}_{\mathcal{A}}(M, N)\right) \text {. }
$$

If $\mathcal{A}$ is an abelian category, we write $\mathrm{C}_{\mathrm{ac}}(\mathcal{A})$ for the full subcategory of $\mathrm{C}(\mathcal{A})$ of acyclic objects. The Verdier quotient $\mathrm{D}(\mathcal{A})=[\mathrm{C}(\mathcal{A})] /\left[\mathrm{C}_{\mathrm{ac}}(\mathcal{A})\right]$ is the derived category of $\mathcal{A}$ and comes with the Verdier localization functor $[\mathrm{C}(\mathcal{A})] \rightarrow \mathrm{D}(\mathcal{A})$. We usually assume that this localization functor is the identity on objects. We abbreviate $\mathrm{D}_{\mathcal{A}}(M, N)=\operatorname{Hom}_{\mathrm{D}(\mathcal{A})}(M, N)$.

3.1.1. Notation. Let $\operatorname{Mod}(\mathrm{R})=\operatorname{Mod}(\mathrm{R}, \mathcal{U})$ be the abelian R-category of $\mathcal{U}$-small Rmodules. The following notation will be used in the rest of this article.

Let $\mathcal{R}:=\mathrm{C}(\mathrm{R}):=\mathrm{C}(\operatorname{Mod}(\mathrm{R}))$ be the category of complexes in $\operatorname{Mod}(\mathrm{R})$ and $\underline{\mathcal{R}}:=$ $\underline{\mathrm{C}}(\mathrm{R}):=\underline{\mathrm{C}}(\operatorname{Mod}(\mathrm{R}))$ the dg R-category of complexes in $\operatorname{Mod}(\mathrm{R})$. Similarly, starting from the field $\mathrm{k}$, we write $\mathcal{K}:=\mathrm{C}(\mathrm{k}):=\mathrm{C}(\operatorname{Mod}(\mathrm{k}))$ and $\underline{\mathcal{K}}:=\underline{\mathrm{C}}(\mathrm{k}):=\underline{\mathrm{C}}(\operatorname{Mod}(\mathrm{k}))$. If relevant, we add the universe as an index and write for example $\mathcal{R}_{\mathcal{U}}$ instead of $\mathcal{R}$.

\subsubsection{Complexes of vector spaces.}

Lemma 3.1. Let $\mathrm{k}$ be a field. Any object of $\mathcal{K}=\mathrm{C}(\mathrm{k})$ is isomorphic to a coproduct of shifts of the two objects $\mathrm{k}$ and Cone $\left(\mathrm{id}_{\mathrm{k}}\right)$.

Proof. Obvious; see Lemma 4.26 for a stronger result.

3.2. Differential graded categories. Note that $\mathcal{R}$ is a closed symmetric monoidal category in the obvious way: the monoidal structure is given by the tensor product $\otimes=\otimes_{\mathrm{R}}$. In particular, we can speak about $\mathcal{R}$-categories (= categories enriched in $\mathcal{R}$ ), see [Kel05]. Note that $\mathcal{R}$-category is just another name for $\mathrm{dg}$ R-category with $\mathcal{U}$-small Hom-sets. We transfer the usual terminology and notation from dg R-categories to $\mathcal{R}$-categories.

We usually denote an $\mathcal{R}$-category by an underlined symbol (e.g. $\underline{\mathcal{N}}$ ) and its underlying ordinary category by a plain symbol (e.g. $\mathcal{M})$. We have already used this convention above: we have the $\mathcal{R}$-category $\underline{\mathrm{C}}(\mathcal{A})$ for an additive R-category $\mathcal{A}$ with $\mathcal{U}$-small Hom-sets (whose underlying category $\mathrm{C}(\mathcal{A})$ was even considered as an $\mathrm{R}$ category), the $\mathcal{R}$-category $\underline{\mathcal{R}}$, and the $\mathcal{K}$-category $\underline{\mathcal{K}}$.

If $\underline{\mathcal{M}}$ is an $\mathcal{R}$-category, we denote its homotopy category by $[\underline{\mathcal{N}}]$. Note that $[\underline{\mathcal{N}}]$ is an R-category.

3.2.1. Dg-enriched arrow categories. For the convenience of the reader we recall the

definition of the arrow category in the $\mathcal{R}$-enriched setting. For $n \in \mathbb{N}$, let $[n]$ denote the free category on the graph $0 \rightarrow 1 \rightarrow \ldots \rightarrow n$. 
Let $\underline{\mathcal{N}}$ be an $\mathcal{R}$-category. The $\mathcal{R}$-category $\underline{\mathcal{N}}^{[1]}$ of arrows in $\mathcal{M}$ has morphisms $A \stackrel{f}{\rightarrow} B$ in $\mathcal{M}$ as objects and morphism objects

$$
\operatorname{Hom}_{\underline{\mathcal{M}}^{[1]}}\left((A \stackrel{f}{\rightarrow} B),\left(A^{\prime} \stackrel{f^{\prime}}{\rightarrow} B^{\prime}\right)\right)=\underline{\mathcal{M}}\left(A, A^{\prime}\right) \underset{\underline{\mathcal{M}}\left(A, B^{\prime}\right)}{\times} \underline{\mathcal{M}}\left(B, B^{\prime}\right) .
$$

For readers more familiar with dg R-categories let us describe this category in the dg language. The dg R-category $\underline{\mathcal{M}}^{[1]}$ has closed degree zero morphisms in $\underline{\mathcal{N}}$ as objects, and given two such objects $A \stackrel{f}{\rightarrow} B$ and $A^{\prime} \stackrel{f^{\prime}}{\rightarrow} B^{\prime}$, the dg R-module of morphisms from $f$ to $f^{\prime}$ is the dg submodule of $\underline{\mathcal{M}}\left(A, A^{\prime}\right) \times \underline{\mathcal{M}}\left(B, B^{\prime}\right)$ consisting of pairs $(a, b)$ making the diagram

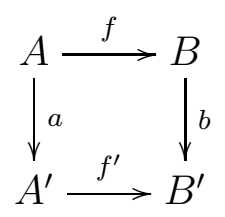

commutative.

The $\mathcal{R}$-category $\underline{\mathcal{M}}^{[2]}$ of two composable arrows is defined similarly. Composition of arrows defines an $\mathcal{R}$-functor

$$
\underline{\mathcal{M}}^{[2]} \rightarrow \underline{\mathcal{M}}^{[1]}
$$

If $\mathcal{A}$ is an additive R-category, taking cones as defined in 3.1 extends naturally to an $\mathcal{R}$-functor Cone: $\underline{\mathrm{C}}(\mathcal{A})^{[1]} \rightarrow \underline{\mathrm{C}}(\mathcal{A})$. Note also that the shift functors $[n]$ come from $\mathcal{R}$-functors $[n]: \underline{\mathrm{C}}(\mathcal{A}) \rightarrow \underline{\mathrm{C}}(\mathcal{A})$.

3.2.2. Categories of $d g$ modules over a $d g$ category. Let $\underline{\mathcal{N}}$ be a $\mathcal{U}$-small $\mathcal{R}$-category. A (right) $\underline{\mathcal{N}}$-module (= a dg $\underline{\mathcal{N}}$-module) is an $\mathcal{R}$-functor $\underline{\mathcal{M}}^{\text {op }} \rightarrow \underline{\mathcal{R}}$. Let $\mathbb{M}$ od $(\underline{\mathcal{N}})$ be the category of $\underline{\mathcal{M}}$-modules and $\underline{\operatorname{Mod}}(\underline{\mathcal{N}})$ the $\mathcal{R}$-category of $\underline{\mathcal{N}}$-modules (note that these categories have $\mathcal{U}$-small Hom-sets and objects in $\mathcal{U}$, by Remark 3.2 below). Given two $\underline{\mathcal{M}}$-modules $M$ and $N$ we write $\operatorname{Mod}_{\underline{\mathcal{M}}}(M, N)=\operatorname{Hom}_{\mathbb{M} \text { od }(\underline{\mathcal{M}})}(M, N)$ and $\underline{\operatorname{Mod}}_{\underline{M}}(M, N)=\operatorname{Hom}_{\underline{\mathbb{M o d}}(\underline{\mathcal{M}})}(M, N)$. There are shift $\mathcal{R}$-functors $[n]: \underline{\mathbb{M o d}}(\underline{\mathcal{M}}) \rightarrow$ $\underline{\operatorname{Mod}}(\underline{\mathcal{M}})$ and a cone $\mathcal{R}$-functor Cone: $\underline{\operatorname{Mod}}(\underline{\mathcal{N}})^{[1]} \rightarrow \underline{\mathbb{M o d}}(\underline{\mathcal{M}})$ defined in the obvious way.

The (enriched) Yoneda functor is the fully faithful $\mathcal{R}$-functor

$$
\begin{aligned}
\mathrm{Yo}: \underline{\mathcal{M}} & \rightarrow \underline{\operatorname{Mod}}(\underline{\mathcal{M}}), \\
M & \mapsto \mathrm{Yo}(M)=\underline{\mathcal{M}}(-, M) .
\end{aligned}
$$

Its underlying functor $\mathcal{M} \rightarrow \operatorname{Mod}(\underline{\mathcal{M}})$ is denoted by the same symbol. The Yoneda functor induces a fully faithful $\mathcal{R}$-functor $\mathrm{Yo}^{[1]}: \underline{\mathcal{M}}^{[1]} \rightarrow \underline{\mathbb{M} o d}(\underline{\mathcal{M}})^{[1]} \operatorname{mapping}(A \stackrel{f}{\rightarrow} B)$ to $(\mathrm{Yo}(A) \stackrel{\mathrm{Yo}(f)}{\longrightarrow} \mathrm{Yo}(B))$. 
Remark 3.2. Let $\mathcal{C}$ and $\mathcal{D}$ be categories and let $\operatorname{Fun}(\mathcal{C}, \mathcal{D})$ be the category of functors from $\mathcal{C}$ to $\mathcal{D}$. Then the following statements are true (see [GR17, Rem. 1.1.12, Cor. 1.4.1.(i)]).

(a) If $\mathcal{C}$ and $\mathcal{D}$ are $\mathcal{U}$-small, so is $\operatorname{Fun}(\mathcal{C}, \mathcal{D})$.

(b) If $\mathcal{C}$ is $\mathcal{U}$-small and $\mathcal{D}$ has $\mathcal{U}$-small Hom-sets, then $\operatorname{Fun}(\mathcal{C}, \mathcal{D})$ has $\mathcal{U}$-small Hom-sets. If, in addition, $\mathcal{D}$ has objects in $\mathcal{U}$, then $\operatorname{Fun}(\mathcal{C}, \mathcal{D})$ has objects in U.

3.2.3. Strongly pretriangulated $d g$ categories. Let $\underline{\mathcal{M}}$ be a $\mathcal{U}$-small $\mathcal{R}$-category.

We say that $\mathcal{M}$ is strongly pretriangulated (resp. pretriangulated) if the following objects are in the essential image of $\mathrm{Yo}: \mathcal{M} \rightarrow \mathbb{M o d}(\underline{\mathcal{M}})(\operatorname{resp} .[\mathrm{Yo}]:[\underline{\mathcal{M}}] \rightarrow$ $[\underline{\operatorname{Mod}}(\underline{\mathcal{M}})])$ :

- the zero module;

- the object $[n] \operatorname{Yo}(M)$, for all objects $M \in \mathcal{M}$ and all $n \in \mathbb{Z}$;

- the object Cone $\left(\mathrm{Yo}^{[1]}(f)\right)$, for all objects $f \in \mathcal{M}^{[1]}$.

We have added the first condition to the definition from [BLL04, 4.3]; it implies that $\mathcal{M}($ resp. $[\underline{\mathcal{N}}])$ has a zero object.

Let $\underline{\mathcal{N}}$ be a strongly pretriangulated $\mathcal{U}$-small $\mathcal{R}$-category. Then there are $\mathcal{R}$-functors "(formal) shift" [1]: $\underline{\mathcal{M}} \rightarrow \underline{\mathcal{M}}$ and "(formal) cone" Cone: $\underline{\mathcal{M}}^{[1]} \rightarrow \underline{\mathcal{M}}$ such that the diagrams

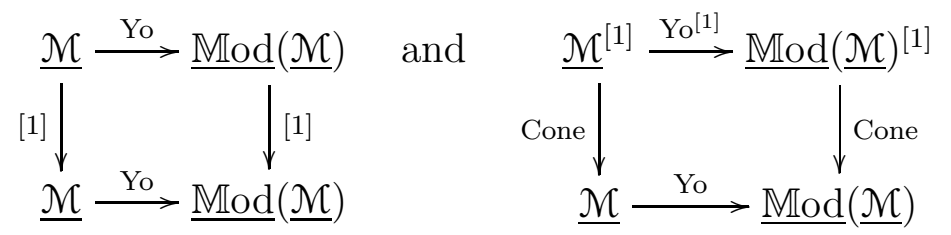

commute up to isomorphisms of $\mathcal{R}$-functors. We fix such isomorphisms so that shift [1] and cone Cone are uniquely determined in the obvious way. We define $[-1]$ similarly, put $[n]=[1]^{n}$ and $[-n]=[-1]^{n}$, for $n \in \mathbb{N}$, and call all these $\mathcal{R}$-functors shift functors. Cone and shift functors commute up to unique isomorphism of $\mathcal{R}$-functors with any $\mathcal{R}$-functor between strongly pretriangulated $\mathcal{R}$-categories.

Example 3.3. The categories $\underline{\mathrm{C}}(\mathcal{A})$, for an additive R-category $\mathcal{A}$, or $\underline{\mathbb{M} o d}(\underline{\mathcal{C}})$, for an $\mathcal{R}$-category $\underline{\mathcal{C}}$, are strongly pretriangulated $\mathcal{R}$-categories, up to size issues: the category $\mathcal{A}$ should be $\mathcal{U}$-small, and $\underline{\mathcal{C}}$ should be $\mathcal{U}^{\prime}$-small, where $\mathcal{U}^{\prime}$ is a universe that is an element of $\mathcal{U}$. We can and will assume that their formal shift and cone functors are the usual functors introduced previously. We make the same assumption for strongly pretriangulated $\mathcal{R}$-subcategories of these categories that are stable under shifts and cones.

Consider the $\mathcal{R}$-functor $\underline{\mathcal{M}} \rightarrow \underline{\mathcal{M}}^{[1]}$ mapping an object $M$ to $M \stackrel{\operatorname{id}_{M}}{\longrightarrow} M$ and a morphism $f: M \rightarrow N$ to $(f, f)$. We define the $\mathcal{R}$-functor iCone, called identity 
cone, to be the composition of this functor with the cone functor, i.e.

$$
\text { iCone } \underline{\mathcal{M}} \rightarrow \underline{\mathcal{M}}^{[1]} \stackrel{\text { Cone }}{\longrightarrow} \underline{\mathcal{M}},
$$

and denote its underlying functor iCone: $\mathcal{M} \rightarrow \mathcal{M}$ by the same symbol. If $f: M \rightarrow N$ is a morphism in $\mathcal{M}$ we have

$$
\operatorname{iCone}(f)=\operatorname{Cone}(f, f): \operatorname{iCone}(M)=\operatorname{Cone}\left(\operatorname{id}_{M}\right) \rightarrow \operatorname{iCone}(N)=\operatorname{Cone}\left(\operatorname{id}_{N}\right) .
$$

Remark 3.4. Given a pretriangulated $\mathcal{R}$-category $\underline{\mathcal{N}}$ we can similarly fix a shift Rfunctor $[1]:[\underline{\mathcal{N}}] \rightarrow[\underline{\mathcal{N}}]$ together with an isomorphism $[1][\mathrm{Yo}] \stackrel{\sim}{\rightarrow}[\mathrm{Yo}][1]$. Then the homotopy category $[\underline{\mathcal{N}}]$ becomes a triangulated R-category in the obvious way. The choice when fixing its shift functor [1] is inessential. Hence the homotopy category of a pretriangulated $\mathcal{R}$-category is canonically triangulated. In fact, we obtain a functor from the category of pretriangulated $\mathcal{R}$-categories to the category of triangulated R-categories.

3.2.4. Dg enhancements. We recall the notion of an $\mathcal{R}$-enhancement (= dg R-enhancement) from [BK90, BLL04, LO10].

Definition 3.5. Let $\mathcal{T}$ be a $\mathcal{U}$-small triangulated R-category. An $\mathcal{R}$-enhancement of $\mathcal{T}$ is a pretriangulated $\mathcal{U}$-small $\mathcal{R}$-category $\underline{\mathcal{E}}$ together with an equivalence $\varepsilon:[\underline{\mathcal{E}}] \stackrel{\sim}{\rightarrow} \mathcal{T}$ of triangulated R-categories (where $[\underline{\mathcal{E}}]$ is considered as a triangulated R-category as explained in Remark 3.4).

3.2.5. Tensors, cotensors, and completeness. An $\mathcal{R}$-category $\mathcal{N}$ is tensored if there is a bifunctor $\odot: \mathcal{M} \times \mathcal{R} \rightarrow \mathcal{M}$ together with natural isomorphisms

$$
\underline{\mathcal{R}}(R, \underline{\mathcal{M}}(M, N)) \cong \underline{\mathcal{M}}(M \odot R, N)
$$

in $R \in \mathcal{R}, M, N \in \mathcal{M}$ (see [Rie14, 3.7]); then the functor $\odot$ is unique up to unique isomorphism. Similarly, $\underline{\mathcal{N}}$ is cotensored if there is a bifunctor $\Psi: \mathcal{R}^{\mathrm{op}} \times \mathcal{M} \rightarrow \mathcal{M}$ together with natural isomorphisms

$$
\underline{\mathcal{R}}(R, \underline{\mathcal{M}}(M, N)) \cong \underline{\mathcal{M}}(M, \Psi(R, N)) .
$$

in $R \in \mathcal{R}, M, N \in \mathcal{M}$; then the functor $\Psi$ is unique up to unique isomorphism. Note that $\odot$ and $\Psi$ come from $\mathcal{R}$-bifunctors (see [Rie14, 3.7.4, 7.3.1]).

Recall from $[$ Rie14, 7.6] that an $\mathcal{R}$-category $\underline{\mathcal{N}}$ is $\mathcal{R}$-bicomplete if and only if it is tensored and cotensored and the underlying category $\mathcal{M}$ is bicomplete (i. e. complete and cocomplete); more precisely, (co)completeness means that all $\mathcal{U}$-small (co)limits exist.

If an $\mathcal{R}$-category $\underline{\mathcal{M}}$ is tensored and strongly pretriangulated, we deduce from (3.11) natural isomorphisms $M \odot 0 \cong 0,([m] M) \odot([r] R) \cong[m+r](M \odot R)$ for $M \in \mathcal{M}$, $R \in \mathcal{R}, m, r \in \mathbb{Z}$. Moreover, we have $M \odot \mathrm{R} \cong M$ and $M \odot \mathrm{iCone}(\mathrm{R}) \cong \mathrm{iCone}(M)$ naturally in $M \in \mathcal{M}$. Also note that $(-\odot-)$ preserves coproducts in each argument. 
3.3. Model categories and $\mathbf{d g}$ enriched functorial factorizations. Let $\underline{\mathcal{M}}$ be an $\mathcal{R}$-category. Recall from $[\mathrm{Rie} 14,12,13]$ that an $\mathcal{R}$-enriched functorial factorization on $\underline{\mathcal{M}}$ is a section of the composition functor (3.6), i. e. an $\mathcal{R}$-functor $\underline{\mathcal{M}}^{[1]} \rightarrow \underline{\mathcal{M}}^{[2]}$ whose composition with (3.6) is the identity. It is convenient to denote an $\mathcal{R}$-enriched functorial factorization by a triple $(L, M, R)$ encoding its effect on objects and morphisms:

$$
\begin{aligned}
(A \stackrel{f}{\rightarrow} B) & \mapsto(A \stackrel{L f}{\longrightarrow} M(f) \stackrel{R f}{\longrightarrow} B), \\
(a, b) & \mapsto(a, M(a, b), b) .
\end{aligned}
$$

If the underlying category $\mathcal{M}$ of $\underline{\mathcal{N}}$ carries a model structure, we say that this model structure admits $\mathcal{R}$-enriched functorial factorizations if there exist $\mathcal{R}$-enriched functorial factorizations $(L, M, R)$ and $\left(L^{\prime}, M^{\prime}, R^{\prime}\right)$ such that $L f$ is a cofibration, $R f$ is a trivial fibration, $L^{\prime} f$ is a trivial cofibration, and $R^{\prime} f$ is fibration, for all objects $f$ of $\underline{\mathcal{M}}^{[1]}$. Then, in particular, there exist $\mathcal{R}$-functors

$$
\text { cof }: \underline{\mathcal{N}} \rightarrow \underline{\mathcal{M}} \text { and } \text { fib }: \underline{\mathcal{N}} \rightarrow \underline{\mathcal{M}}
$$

together with $\mathcal{R}$-natural transformations cof $\stackrel{\gamma}{\rightarrow}$ id $\stackrel{\phi}{\rightarrow}$ fib such that for each $M \in \underline{\mathcal{M}}$ the object $\operatorname{cof}(M)$ is cofibrant and the morphism $\gamma_{M}: \operatorname{cof}(M) \rightarrow M$ is a trivial fibration, and $\mathrm{fib}(M)$ is fibrant and $\phi_{M}: M \rightarrow \mathrm{fib}(M)$ is a trivial cofibration. We call any such pair (cof, $\gamma$ ) (resp. (fib, $\phi)$ ) an R-enriched cofibrant (resp. fibrant) resolution functor and call cof (resp. fib) an $\mathcal{R}$-enriched cofibrant (resp. fibrant) replacement functor.

Note that the notion of a model category depends on a universe: a model category with respect to the universe $\mathcal{U}$ has $\mathcal{U}$-small Hom-sets and all $\mathcal{U}$-small (co)limits.

Theorem 3.6. Let $\mathrm{k}$ be a field. Let $\underline{\mathcal{N}}$ be a strongly pretriangulated $\mathcal{K}$-bicomplete $\mathcal{K}$ category. Assume that the underlying category $\mathcal{M}$ is equipped with a model structure turning it into a cofibrantly generated model category (with respect to the universe U) (so it admits the small object argument) such that the sets of cofibrations and of trivial cofibrations are stable

(a) under taking shifts, i.e. mapping $i: E \rightarrow F$ to $[n] i:[n] E \rightarrow[n] F$, for all $n \in \mathbb{Z}$, and

(b) under applying the functor iCone: $\mathcal{M} \rightarrow \mathcal{M}$, i. e. under mapping $i: E \rightarrow F$ to $\mathrm{iCone}(i): \operatorname{iCone}(E) \rightarrow \mathrm{iCone}(F)$ (see (3.9)).

Then this model structure admits $\mathcal{K}$-enriched functorial factorizations.

The assumption that $\mathrm{k}$ is a field is essential as we explain in 4.1.4.

Proof. This follows from [Rie14, Cor. 13.2.4] and the following Lemma 3.7. Note that $\mathcal{K}$-bicompleteness of $\underline{\mathcal{N}}$ implies that $\underline{\mathcal{N}}$ is tensored (see 3.2.5). 
Lemma 3.7. Let $\mathrm{k}$ be a field. Let $\underline{\mathcal{M}}$ be a strongly pretriangulated tensored $\mathcal{K}$-category, with tensored structure given by the bifunctor $\odot$. Assume that the underlying category $\mathcal{M}$ is equipped with a model structure turning it into a model category such that the set of cofibrations (resp. of trivial cofibrations) is stable under all shifts and under the functor iCone $(-)$. Then, for each $K \in \mathcal{K}$, the functor $(-\odot K): \mathcal{M} \rightarrow \mathcal{M}$ preserves cofibrations (resp. trivial cofibrations).

Proof. Lemma 3.1 tells us that any object of $\mathcal{K}$ is a coproduct of shifts of $\mathrm{k}$ and Cone $\left(\mathrm{id}_{\mathrm{k}}\right)=\mathrm{iCone}(\mathrm{k})$. Since $(-\odot-)$ commutes with coproducts and shifts in each argument and since the set of (trivial) cofibrations in $\mathcal{M}$ is closed under coproducts and shifts it is enough to prove the claim in the two cases $K=\mathrm{k}$ and $K=\mathrm{iCone}(\mathrm{k})$. The claim for $K=\mathrm{k}$ is trivial because $(-\odot \mathrm{k}) \cong \mathrm{id}$, and the claim for $K=\mathrm{iCone}(\mathrm{k})$ is clear by assumption because $(-\odot \mathrm{iCone}(\mathrm{k})) \cong \mathrm{iCone}(-)$.

Remark 3.8. In most of our applications of Theorem 3.6, $\underline{\mathcal{N}}$ is some category $\underline{\mathrm{C}}(\mathcal{A})$ of complexes in an abelian k-category $\mathcal{A}$ with $\mathcal{U}$-small Hom-sets, the weak equivalences of the considered model structure on $\mathcal{M}=\mathrm{C}(\mathcal{A})$ are the quasi-isomorphisms, and the cofibrations and trivial cofibrations are obviously stable under all shifts. In this case the functor iCone maps any morphism to a quasi-isomorphism because it maps any object to a contractible and hence acyclic object. Therefore it is enough to check that the cofibrations are stable under the functor iCone(-). In Lemma 3.9 we give a criterion ensuring this condition.

Note that the notion of a Grothendieck abelian category depends on a universe: a Grothendieck abelian category with respect to the universe $\mathcal{U}$ has $\mathcal{U}$-small Hom-sets, has $\mathcal{U}$-small coproducts, and all $\mathcal{U}$-small filtered colimits are exact.

Lemma 3.9. Let $\mathcal{A}$ be a Grothendieck abelian category (with respect to the universe $\mathcal{U}$ ) and let a model structure on $\mathrm{C}(\mathcal{A})$ be given turning it into a model category whose weak equivalences are the quasi-isomorphisms. Assume that the cofibrant objects are stable under all shifts and that a morphism is a cofibration if and only if it is a monomorphism with cofibrant cokernel (resp. a degreewise split monomorphism with cofibrant cokernel). Then iCone maps cofibrations to trivial cofibrations.

Proof. Let $i: A \rightarrow B$ be a cofibration. The obvious commutative diagram

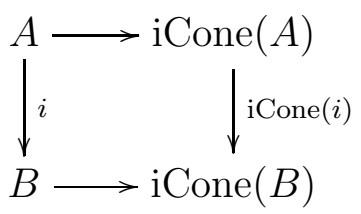

induces a morphism

$$
B \bigsqcup_{A} \mathrm{iCone}(A) \rightarrow \mathrm{iCone}(B) .
$$


Since $i$ is a monomorphism we have $B \bigsqcup_{A} \operatorname{iCone}(A)=\operatorname{Cone}(i)$. We obtain the factorization

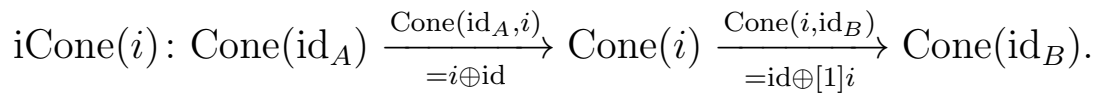

The morphism Cone $\left(\mathrm{id}_{A}, i\right)$ is a pushout of $i$ and hence a cofibration. The morphism Cone $\left(i, \mathrm{id}_{B}\right)$ is a cofibration because it is a monomorphism (resp. degreewise split monomorphism) whose cokernel is the cofibrant object $[1] \operatorname{cok}(i)$. This implies that $\mathrm{iCone}(i)$ is a cofibration. It is trivial because source and target are contractible.

Corollary 3.10. Under the assumptions of Lemma 3.9, given a (trivial) cofibration $i: A \rightarrow B$, the induced morphism $B \bigsqcup_{A} \mathrm{iCone}(A) \rightarrow \mathrm{iCone}(B)$ is a (trivial) cofibration.

Proof. Clear from the proof of Lemma 3.9. Note that $B \bigsqcup_{A} \mathrm{iCone}(A)=\operatorname{Cone}(i)$ is acyclic if $i$ is trivial.

\section{Applications}

We recall some model structures and provide several examples where Theorem 3.6 is applicable. The examples concerning modules over dg k-categories in 4.3 and sheaves on k-ringed spaces in 4.4 are not relevant for the rest of this article. Let $\mathcal{U} \in \mathcal{V}$ be universes.

4.1. Grothendieck abelian categories. Let $\mathcal{A}$ be a Grothendieck abelian category with respect to the universe $\mathcal{U}$. Then $\mathrm{C}(\mathcal{A})$ is a Grothendieck abelian category; in particular, it is bicomplete.

4.1.1. Injective model structure. Recall that a complex $I$ in $\mathcal{A}$ is called h-injective if $\left[\underline{\mathrm{C}}_{\mathcal{A}}\right](A, I)=0$ for any acyclic complex $A$ in $\mathcal{A}$. The injective model structure or I-model structure on $\mathrm{C}(\mathcal{A})$ (considered as an ordinary category) is the following triple of sets of morphisms in $C(\mathcal{A})$ (see [Bek00, Prop. 3.13] or [Gil07, Cor. 7.1]).

- the weak equivalences are the quasi-isomorphisms;

- the cofibrations are the monomorphisms;

- the fibrations are the epimorphisms with h-injective and componentwise injective kernel.

This model structure turns $\mathrm{C}(\mathcal{A})$ into a cofibrantly generated model category. We call the (co)fibrations and (co)fibrant objects of this model structure $\mathbb{I}$-(co)fibrations and $\mathbb{I}$-(co)fibrant objects, respectively. The $\mathbb{I}$-fibrant objects are precisely the h-injective and componentwise injective objects of $\mathrm{C}(\mathcal{A})$; they are sometimes called dg-injective. Let $\mathbb{I}(\mathcal{A}) \subset \underline{\mathrm{C}}(\mathcal{A})$ denote the full subcategory of these objects. It is a strongly pretriangulated $\mathrm{C}(\mathbb{Z})$-category. All objects of $\mathrm{C}(\mathcal{A})$ are $\mathbb{I}$-cofibrant.

Remark 4.1. Right derived functors can be computed using I-fibrant resolutions. 
Lemma 4.2. Let $\mathcal{A}$ be a Grothendieck abelian category. Then iCone maps $\mathbb{I}$-cofibrations to trivial $\mathbb{I}$-cofibrations.

Proof. Immediate from Lemma 3.9.

4.1.2. Injective enhancements. Let $\mathrm{R}$ be a commutative ring and $\mathcal{A}$ a Grothendieck abelian R-category. The strongly pretriangulated $\mathcal{R}$-category $\mathbb{I}(\mathcal{A})$ together with the obvious equivalence

$$
[\underline{\mathbb{I}}(\mathcal{A})] \stackrel{\sim}{\rightarrow} \mathrm{D}(\mathcal{A})
$$

of triangulated R-categories is an $\mathcal{R}$-enhancement of $\mathrm{D}(\mathcal{A})$. We call it the injective enhancement or $\mathbb{I}$-enhancement of $\mathrm{D}(\mathcal{A})$.

4.1.3. Dg k-enriched functorial factorizations for Grothendieck abelian k-categories.

Theorem 4.3. Let $\mathcal{A}$ be a Grothendieck abelian k-category where $\mathrm{k}$ is a field. Assume that the $\mathcal{K}$-category $\mathrm{C}(\mathcal{A})$ is $\mathcal{K}$-bicomplete. Then the $\mathbb{I}$-model structure on $\mathrm{C}(\mathcal{A})$ admits $\mathcal{K}$-enriched functorial factorizations.

Proof. Obviously, $\underline{\mathrm{C}}(\mathcal{A})$ is strongly pretriangulated. The $\mathbb{I}$-model structure on $\mathrm{C}(\mathcal{A})$ is cofibrantly generated and its (trivial) cofibrations are stable under all shifts. Together with Lemma 4.2 this shows that all assumptions of Theorem 3.6 are satisfied.

4.1.4. A counterexample. Let $\operatorname{Mod}(\mathbb{Z})$ be the category of $\mathcal{U}$-small abelian groups. We consider the injective model structure on $\mathrm{C}(\mathbb{Z})=\mathrm{C}(\operatorname{Mod}(\mathbb{Z}))$. Let $\mathbb{I}(\mathbb{Z}) \subset \mathrm{C}(\mathbb{Z})$ be the subcategory of $\mathbb{I}$-fibrant objects. The categories $\mathrm{C}(\mathrm{Ab})$ and $\mathbb{I}(\mathrm{Ab})$ are additive.

Note that $\underline{\mathrm{C}}(\mathbb{Z})$ is $\underline{\mathrm{C}}(\mathbb{Z})$-bicomplete (e.g. by Lemma 4.19 below). Therefore the following result shows that Theorems 3.6 and 4.3 with the field $\mathrm{k}$ replaced by the integers $\mathbb{Z}$ are not true.

Lemma 4.4. There is no pair $(\mathbf{i}, \mathfrak{l})$ where $\mathbf{i}$ is an additive functor $\mathrm{C}(\mathbb{Z}) \rightarrow \mathbb{I}(\mathbb{Z})$ and $\iota$ is a natural transformation id $\rightarrow \mathbf{i}$ of functors such that for each $M \in \mathrm{C}(\mathbb{Z})$ the morphism $\mathfrak{\iota}_{M}: M \rightarrow \mathbf{i}(M)$ is a quasi-isomorphism (cf. 3.3).

In particular, the $\mathbb{I}$-model structure on $\mathrm{C}(\mathbb{Z})$ does not admit $\mathrm{C}(\mathbb{Z})$-enriched functorial factorizations, and there is no $\mathrm{C}(\mathbb{Z})$-enriched fibrant replacement functor.

Proof. Assume that such a pair $(\mathbf{i}, \mathfrak{l})$ exists. Consider $T:=\mathbb{Z} / 2 \mathbb{Z}$ as an object of $\mathrm{C}(\mathbb{Z})$ sitting in degree zero. Then

$$
2 \mathrm{id}_{\mathbf{i}(T)}=2 \mathbf{i}\left(\mathrm{id}_{T}\right)=\mathbf{i}\left(2 \mathrm{id}_{T}\right)=\mathbf{i}(0)=0,
$$

so multiplication by 2 is the zero map on $\mathbf{i}(T)$. Since all components of $\mathbf{i}(T)$ are injective and therefore divisible, multiplication by 2 is surjective on $\mathbf{i}(T)$. Hence $\mathbf{i}(T)=0$ contradicting the assumption that $\mathfrak{\iota}_{T}$ is a quasi-isomorphism.

Remark 4.5. The above proof essentially boils down to the fact that there is no additive functor $\operatorname{Mod}(\mathbb{Z}) \rightarrow \operatorname{Mod}(\mathbb{Z})^{[1]}$ mapping an abelian group $A$ to a monomorphism $A \hookrightarrow I_{A}$ into an injective abelian group $I_{A}$ (see [Sch16, Lemma 3]). 
4.1.5. Drinfeld quotients. We use the name Drinfeld quotient for Drinfeld's notion of a quotient in [Dri08, 1.2].

Lemma 4.6. Let $\underline{\mathcal{A}}$ be a pretriangulated $\mathcal{U}$-small $\mathcal{R}$-category and $\underline{\mathcal{B}}$ a full pretriangulated $\mathcal{R}$-subcategory. Let $F: \underline{\mathcal{A}} \rightarrow \underline{\mathcal{C}}$ be an $\mathcal{R}$-functor to a pretriangulated $\mathcal{U}$-small $\mathcal{R}$-category $\underline{\mathrm{C}}$. Then the following statements are equivalent:

(a) the functor $[F]:[\underline{\mathcal{A}}] \rightarrow[\underline{\mathcal{C}}]$ of triangulated $\mathrm{R}$-categories (see Remark 3.4) vanishes on $[\underline{\mathcal{B}}]$ and the induced functor $[\underline{\mathcal{A}}] /[\underline{\mathcal{B}}] \rightarrow[\underline{\mathcal{C}}]$ is an equivalence of triangulated R-categories;

(b) the functor $F: \underline{\mathcal{A}} \rightarrow \underline{\mathcal{C}}$ (more precisely, the diagram $\underline{\mathcal{A}} \stackrel{\text { id }}{\leftarrow} \underline{\mathcal{A}} \stackrel{F}{\rightarrow} \underline{\mathcal{C}}$ ) is a Drinfeld quotient of $\underline{\mathcal{A}}$ by $\underline{\mathcal{B}}$.

Proof. Clear from the definition of a Drinfeld quotient.

Recall the universe $\mathcal{V}$ that contains $\mathcal{U}$ as an element.

Lemma 4.7. Let $\mathrm{k}$ be a field and let $\mathcal{A}$ be a Grothendieck abelian k-category (with respect to the universe $\mathcal{U}$ ) with objects in $\mathcal{U}$, and assume that $\underline{\mathrm{C}}(\mathcal{A})$ is $\mathcal{K}$-bicomplete. Let $\mathbf{i}: \underline{\mathrm{C}}(\mathcal{A}) \rightarrow \underline{I}(\mathcal{A})$ be a $\mathcal{K}$-enriched $\mathbb{I}$-fibrant replacement functor (which exists by Theorem 4.3). Then $\mathbf{i}$ is a Drinfeld quotient of the pretriangulated $\mathcal{V}$-small $\mathcal{K}$-category $\underline{\mathrm{C}}(\mathcal{A})$ by its full $\mathcal{K}$-subcategory $\underline{\mathrm{C}}_{\mathrm{ac}}(\mathcal{A})$ of acyclic objects. In particular, $\mathbf{i}$ induces a unique equivalence

$$
\overline{[\mathbf{i}]}: \mathrm{D}(\mathcal{A}) \stackrel{\sim}{\rightarrow}[\underline{\mathbb{I}}(\mathcal{A})]
$$

of triangulated $\mathbf{k}$-categories such that the diagram

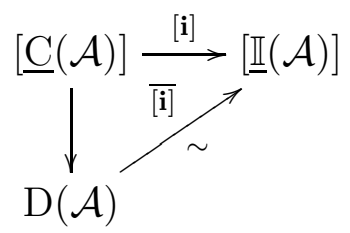

commutes. This equivalence has (4.1) as a quasi-inverse.

Proof. The $\mathcal{K}$-categories $\underline{\mathrm{C}}(\mathcal{A}), \underline{\mathrm{C}}_{\mathrm{ac}}(\mathcal{A}), \underline{\mathbb{I}}(\mathcal{A})$ are clearly $\mathcal{V}$-small (since they have objects in $\mathcal{U}$ ) and pretriangulated. Since an acyclic h-injective complex is contractible, $[\mathbf{i}]:[\underline{\mathrm{C}}(\mathcal{A})] \rightarrow[\underline{\mathbb{I}}(\mathcal{A})]$ maps acyclic objects to zero objects, and therefore induces a unique functor $\overline{[\mathbf{i}]}: \mathrm{D}(\mathcal{A})=[\underline{\mathrm{C}}(\mathcal{A})] /\left[\underline{\mathrm{C}}_{\mathrm{ac}}(\mathcal{A})\right] \rightarrow[\underline{\mathbb{}}(\mathcal{A})]$ by the universal property of the Verdier quotient. The usual construction of a quasi-inverse to a fully faithful essentially surjective functor can be used to show that $\overline{[\mathbf{i}]}$ is quasi-inverse to (4.1). Now apply Lemma 4.6 using the universe $\mathcal{V}$.

4.2. Ringed sites. Let $\left(\mathcal{X}, \mathcal{O}=\mathcal{O}_{\mathcal{X}}\right)$ be a ringed site [SP16, 04KQ]. We assume that it is $\mathcal{U}$-small, i. e. its underlying category is $\mathcal{U}$-small, all coverings of objects are $\mathcal{U}$ sets, and the sheaf $\mathcal{O}_{\mathcal{X}}$ takes values in the category of $\mathcal{U}$-small commutative rings. We write $\operatorname{Sh}(\mathcal{X})$ for the category of sheaves of $\mathcal{U}$-small sets on the site $\mathcal{X}$. The category 
$\operatorname{Mod}(\mathcal{X})$ of sheaves of $\mathcal{U}$-small $\mathcal{O}$-modules has $\mathcal{U}$-small Hom-sets and objects in $\mathcal{U}$ (by Remark 3.2) and is a Grothendieck abelian category [SP16, 07A5] with respect to the universe $\mathcal{U}$. We usually say $\mathcal{O}$-module instead of sheaf of $\mathcal{U}$-small $\mathcal{O}$-modules. We abbreviate $\operatorname{Hom}_{\mathcal{X}}=\operatorname{Hom}_{\operatorname{Mod}(\mathcal{X})}$ and $\otimes=\otimes_{\mathcal{O}}$ and $\mathcal{H}$ om $=\mathcal{H}$ om $m_{\mathcal{O}}$, and write $\mathrm{C}(\mathcal{X})$ instead of $\mathrm{C}(\operatorname{Mod}(\mathcal{X}))$, and $\mathrm{C}_{\mathcal{X}}(M, N)=\mathrm{C}_{\operatorname{Mod}(\mathcal{X})}(M, N)$, and similarly for $\underline{\mathrm{C}}(\mathcal{X})$, $[\underline{\mathrm{C}}(\mathcal{X})]$, and $\mathrm{D}(\mathcal{X})$. Sometimes we write $\operatorname{Mod}(\mathcal{X}, \mathcal{O}), \ldots$ instead of $\operatorname{Mod}(\mathcal{X}), \ldots$ in order to avoid ambiguity. All sites and ringed sites in the rest of this subsection are assumed to be $\mathcal{U}$-small.

4.2.1. Injective model structure. If $(\mathcal{X}, \mathcal{O})$ is a ringed site, $\mathrm{C}(\mathcal{X})$ carries the injective model structure from 4.1.1. We abbreviate $\mathbb{I}(\mathcal{X})=\underline{\mathbb{I}}(\operatorname{Mod}(\mathcal{X}))$.

4.2.2. Extension-by-zero model structure. Following [CD09] we introduce a model structure on $\mathrm{C}(\mathcal{X})$ which is useful for computing derived tensor products and derived pullbacks.

Let $(\mathcal{X}, \mathcal{O})$ be a ringed site. For $U \in \mathcal{X}$ the continuous and cocontinuous functor $j_{U}: \mathcal{X} / U \rightarrow \mathcal{X}$ [SP16,00XZ] gives rise to a morphism of ringed topoi

$$
\left(j_{U}, j_{U}^{\sharp}:=\mathrm{id}\right):\left(\operatorname{Sh}(\mathcal{X} / U), \mathcal{O}_{U}:=\mathcal{O}_{\mathcal{X} / U}:=j^{-1} \mathcal{O}\right) \rightarrow(\operatorname{Sh}(\mathcal{X}), \mathcal{O}) .
$$

The extension by zero $j_{U !} \mathcal{O}_{U} \in \operatorname{Mod}(\mathcal{X})$ is a flat $\mathcal{O}$-module [SP16, 03EV].

Theorem 4.8. Let $(\mathcal{X}, \mathcal{O})$ be a ringed site. There is a model structure on $\mathrm{C}(\mathcal{X})$ turning it into a cofibrantly generated model category whose weak equivalences are the quasi-isomorphisms and whose cofibrations are the smallest set of morphisms in $\mathrm{C}(\mathcal{X})$ that contains all morphisms of the form

$$
[n] j_{U !} \mathcal{O}_{U} \rightarrow \mathrm{iCone}\left([n] j_{U !} \mathcal{O}_{U}\right), \quad \text { for } U \in \mathcal{X} \text { and } n \in \mathbb{Z},
$$

and is closed under pushouts, retracts, and transfinite compositions.

Proof. This follows from [CD09, Ex. 2.3, Thm. 2.5]. Note that the modern definition of a hypercover used there (see e.g. [DHI04]) does not require that the site has fiber products.

We call the model structure of Theorem 4.8 the extension-by-zero model structure or $\mathbb{E}$-model structure and call its (co)fibrations and (co)fibrant objects $\mathbb{E}$ (co)fibrations and $\mathbb{E}$-(co)fibrant objects, respectively. Note that $j_{U !} \mathcal{O}_{U}$ and all its shifts are $\mathbb{E}$-cofibrant. Let $\underline{\mathbb{E}}(\mathcal{X}) \subset \underline{\mathrm{C}}(\mathcal{X})$ denote the full subcategory of $\mathbb{E}$-cofibrant objects.

Lemma 4.9. Let $(\mathcal{X}, \mathcal{O})$ be a ringed site. Then iCone maps $\mathbb{E}$-cofibrations to trivial $\mathbb{E}$-cofibrations.

Proof. Let $i: A \rightarrow B$ be an $\mathbb{E}$-cofibration. Consider the factorization

$$
\mathrm{iCone}(i): \mathrm{iCone}(A) \rightarrow B \bigsqcup_{A} \mathrm{iCone}(A) \rightarrow \mathrm{iCone}(B)
$$


obtained from the commutative diagram (3.15). As a pushout of an $\mathbb{E}$-cofibration, the first morphism is a $\mathbb{E}$-cofibration. The second morphism is an $\mathbb{E}$-cofibration, by the proof of [CD09, Lemma 2.7]. Therefore the composition is an $\mathbb{E}$-cofibration; it has acyclic source and target and is therefore trivial.

Lemma 4.10. Let $(\mathcal{X}, \mathcal{O})$ be a ringed site. $A$ morphism $A \rightarrow B$ in $\mathrm{C}(\mathcal{X})$ is an $\mathbb{E}$-cofibration if and only if it is a degreewise split monomorphism with $\mathbb{E}$-cofibrant cokernel.

Proof. The set of all degreewise split monomorphisms in $\mathrm{C}(\mathcal{X})$ is closed under pushouts, retracts, and transfinite compositions (by Grothendieck's AB5 condition). Since it contains all morphisms in (4.6) any $\mathbb{E}$-cofibration is a degreewise split monomorphism. If $i: A \rightarrow B$ is an $\mathbb{E}$-cofibration, its pushout along $A \rightarrow 0$ is the $\mathbb{E}$-cofibration $0 \rightarrow \operatorname{cok}(i)$. Hence $\operatorname{cok}(i)$ is $\mathbb{E}$-cofibrant.

Conversely, let $i: A \rightarrow B$ be a degreewise split monomorphism with $\mathbb{E}$-cofibrant cokernel $\operatorname{cok}(i)$. The shift $C:=[-1] \operatorname{cok}(i)$ is also $\mathbb{E}$-cofibrant. Since $i$ is degreewise split, $i$ is isomorphic to the obvious inclusion $A \hookrightarrow A \oplus[1] C$ in $\operatorname{Mod}(\mathcal{X})^{\mathbb{Z}}$, and there is a morphism $m: C \rightarrow A$ in $\mathrm{C}(\mathcal{X})$ such that $i$ is isomorphic to the canonical morphism $A \rightarrow \operatorname{Cone}(m)$. But

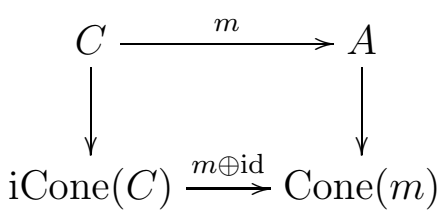

is a pushout diagram whose left vertical arrow is an $\mathbb{E}$-cofibration: in the proof of Lemma 4.9 we have seen that the morphism $B^{\prime} \bigsqcup_{A^{\prime}} \mathrm{i}$ Cone $\left(A^{\prime}\right) \rightarrow \mathrm{i} \operatorname{Cone}\left(B^{\prime}\right)$ is an $\mathbb{E}$ cofibration if $A^{\prime} \rightarrow B^{\prime}$ is an $\mathbb{E}$-cofibration; apply this to $0 \rightarrow C$. This implies that $A \rightarrow \operatorname{Cone}(m)$ is an $\mathbb{E}$-cofibration, and so is $i$.

Lemma 4.11. Let $(\mathcal{X}, \mathcal{O})$ be a ringed site. If $E$ is an $\mathbb{E}$-cofibrant object, then $E$ is an h-flat complex of $\mathcal{O}$-modules with flat components.

Proof. All morphisms in (4.6) are degreewise split monomorphisms with h-flat and componentwise flat cokernel $[n+1] j_{U} \mathcal{O}_{U}$. Therefore the set of all degreewise split monomorphisms $A \stackrel{m}{\rightarrow} B$ in $\mathrm{C}(\mathcal{X})$ such that $\operatorname{cok}(m)$ is h-flat and componentwise flat contains all morphisms in (4.6). Moreover, it is closed under pushouts, retracts, and transfinite compositions (by Grothendieck's AB5 condition). Therefore it contains all $\mathbb{E}$-cofibrations and in particular $0 \rightarrow E$.

Remark 4.12. Lemma 4.11 implies that derived tensor products and derived pullbacks can be computed using $\mathbb{E}$-cofibrant resolutions, cf. Propositions A.13 and A.14. 
4.2.3. The final topos. Let pt be the punctual site. Sheaves and presheaves of $\mathcal{U}$-small sets on pt are just $\mathcal{U}$-small sets. Let $\mathcal{X}$ be a site. Consider the morphism of topoi

$$
\sigma=\left(\sigma^{-1}, \sigma_{*}\right): \operatorname{Sh}(\mathcal{X}) \rightarrow \operatorname{Sh}(\mathrm{pt})=\operatorname{Setsu}
$$

where $\sigma^{-1}: \operatorname{Sets} \mathfrak{u}=\operatorname{Sh}(\mathrm{pt}) \rightarrow \operatorname{Sh}(\mathcal{X})$ is the exact and colimit preserving functor that maps a set $S$ to the constant sheaf $\sigma^{-1} S$ with value $S$, and where $\sigma_{*}=\Gamma=$ $\Gamma(\mathcal{X},-): \operatorname{Sh}(\mathcal{X}) \rightarrow \operatorname{Sh}(\mathrm{pt})=$ Setsu is the global section functor, see [SP16, 06UN]. Up to unique isomorphism, $\sigma$ is the unique morphism of topoi $\operatorname{Sh}(\mathcal{X}) \rightarrow \operatorname{Sh}(p t)=\operatorname{Sets} u$, by [SGA4i, Exp. IV, 4.3].

4.2.4. Ringed sites and topoi over a ring. Let $\mathrm{R}$ be a commutative ring.

Definition 4.13. An R-ringed site or ringed site over $\mathrm{R}$ is a ringed site $(\mathcal{X}, \mathcal{O})$ such that $\mathcal{O}$ is a sheaf of commutative R-algebras. The category of R-ringed sites is defined in the obvious way, cf. [SP16, 04KQ].

For example, the ringed site (pt, $\mathrm{R}$ ) is an $\mathrm{R}$-ringed site. A sheaf of $\mathcal{U}$-small $\mathrm{R}$ modules on $(\mathrm{pt}, \mathrm{R})$ is a $\mathcal{U}$-small $\mathrm{R}$-module, so $\operatorname{Mod}(\mathrm{pt}, \mathrm{R})=\operatorname{Mod}(\mathrm{R})$ and $\mathrm{C}(\mathrm{pt})=\mathcal{R}=$ $\mathrm{C}(\mathrm{R})$.

If $(\mathcal{X}, \mathcal{O})$ is an R-ringed site there is a canonical morphism $\sigma^{\sharp}: \sigma^{-1} \mathrm{R} \rightarrow \mathcal{O}$ of sheaves of rings turning (4.9) into a morphism

$$
\left(\sigma, \sigma^{\sharp}\right):(\operatorname{Sh}(\mathcal{X}), \mathcal{O}) \rightarrow(\mathrm{Sh}(\mathrm{pt}), \mathrm{R})
$$

of ringed topoi. Conversely, if $(\mathcal{X}, \mathcal{O})$ is a ringed site and $\rho: \sigma^{-1} \mathrm{R} \rightarrow \mathcal{O}$ is a morphism of sheaves of rings, then $(\mathcal{X}, \mathcal{O})$ becomes canonically an R-ringed site. These two constructions are obviously inverse to each other.

Definition 4.14. An R-ringed topos or ringed topos over $\mathrm{R}$ is a pair $(\operatorname{Sh}(\mathcal{X}), \mathcal{O})$ where $(\mathcal{X}, \mathcal{O})$ is a R-ringed site. The category of R-ringed topoi is defined in the obvious way, cf. [SP16, 01D3].

4.2.5. Enriched completeness. If $(\mathcal{X}, \mathcal{O})$ is a ringed site over $\mathrm{R}$ then $\underline{\mathrm{C}}(\mathcal{X})$ is an $\mathcal{R}$ category with underlying category $\mathrm{C}(\mathcal{X})$.

Lemma 4.15. Let $(\mathcal{X}, \mathcal{O})$ be a ringed site over $\mathrm{R}$. Then $\underline{\mathrm{C}}(\mathcal{X})$ is tensored and cotensored, and $\mathcal{R}$-bicomplete.

Proof. The morphism (4.10) of ringed topoi associated to $(\mathcal{X}, \mathcal{O})$ gives rise to an adjoint pair $\left(\sigma^{*}, \sigma_{*}\right)$ of $\mathcal{R}$-functors $\sigma^{*}: \underline{\mathcal{R}} \rightleftarrows \underline{\mathrm{C}}(\mathcal{X}): \sigma_{*}$. Using this adjunction and the $(\otimes, \mathcal{H o m})$-adjunction we obtain natural isomorphisms

$$
\underline{\mathcal{R}}\left(R, \sigma_{*} \mathcal{H o m}(M, N)\right) \cong \underline{\mathrm{C}}_{\mathcal{X}}\left(M \otimes \sigma^{*} R, N\right) \cong \underline{\mathrm{C}}_{\mathcal{X}}\left(M, \mathcal{H o m}\left(\sigma^{*} R, N\right)\right)
$$

in $R \in \mathcal{R}$ and $M, N \in \mathrm{C}(\mathcal{X})$. Observe that

$$
\underline{\mathrm{C}}_{\mathcal{X}}(M, N)=\sigma_{*} \mathcal{H o m}(M, N) .
$$


Therefore the isomorphisms (4.11) show that the $\mathcal{R}$-category $\underline{\mathrm{C}}(\mathcal{X})$ is tensored and cotensored (cf. (3.11), (3.12)). Note that $\mathrm{C}(\mathcal{X})$ is a Grothendieck abelian category and therefore bicomplete. This implies that $\underline{\mathrm{C}}(\mathcal{X})$ is $\mathcal{R}$-bicomplete, by 3.2.5.

Remark 4.16. The fact that $\underline{\mathrm{C}}(\mathcal{X})$ is tensored and cotensored can more formally be deduced as follows. The tensor product $\otimes$ turns both categories $\mathrm{C}(\mathcal{X})$ and $\mathcal{R}$ into closed symmetric monoidal categories. In particular, these two categories are enriched, tensored and cotensored over themselves. The functor $\sigma^{*}: \mathcal{R} \rightarrow \mathrm{C}(\mathcal{X})$ is strong monoidal by [SP16, 03EL]. Therefore $\mathrm{C}(\mathcal{X})$ canonically becomes a tensored and cotensored $\mathcal{R}$-category $\underline{\mathrm{C}}(\mathcal{X})$, by [Rie14, Thm. 3.7.11].

\subsubsection{Dg k-enriched functorial factorizations for $\mathrm{k}$-ringed sites.}

Theorem 4.17. Let $(\mathcal{X}, \mathcal{O})$ be a $\mathcal{U}$-small ringed site over a field $\mathrm{k}$. Then the $\mathbb{I}$-model structure and the $\mathbb{E}$-model structure on $\mathrm{C}(\mathcal{X})$ admit $\mathcal{K}$-enriched functorial factorizations.

Proof. Obviously, $\underline{\mathrm{C}}(\mathcal{X})$ is strongly pretriangulated. It is $\mathcal{K}$-bicomplete by Lemma 4.15. The claim for the $\mathbb{I}$-model structure follows from Theorem 4.3, and the same line of argument works for the $\mathbb{E}$-model structure: it is cofibrantly generated, its (trivial) cofibrations are stable under all shifts, and Lemma 4.9 holds; therefore Theorem 3.6 applies.

4.3. Modules over dg categories. Recall our notation for module categories over $\mathcal{R}$-categories from 3.2.2.

4.3.1. Model structures. Let $\underline{\mathcal{e}}$ be an $\mathcal{R}$-category. We always assume that it is $\mathcal{U}$-small in the rest of this subsection. The category $\operatorname{Mod}(\underline{\mathcal{C}})$ has $\mathcal{U}$-small Hom-sets, objects in $\mathcal{U}$, and all $\mathcal{U}$-small colimits and limits. It has two well-known model structures whose weak equivalences are the quasi-isomorphisms ([Kel06, Thm. 3.2], [LS14, Thm. 2.2]):

(a) The injective model structure whose cofibrations are the monomorphisms. We call this model structure the $\mathbb{I}$-model structure and its (co)fibrations and (co)fibrant objects $\mathbb{I}$-(co)fibrations and $\mathbb{I}$-(co)fibrant objects, respectively.

(b) The projective model structure whose fibrations are the epimorphisms. We call this model structure the $\mathbb{P}$-model structure and its (co)fibrations and (co)fibrant objects $\mathbb{P}_{-}$(co)fibrations and $\mathbb{P}_{-}$(co)fibrant objects, respectively.

Each of these model structures turns $\operatorname{Mod}(\underline{\mathcal{C}})$ into a cofibrantly generated model category. Any $\mathbb{I}$-fibrant object is h-injective, and all objects are $\mathbb{I}$-cofibrant. Any $\mathbb{P}$-cofibrant object is h-projective and h-flat ([LS14, Lemmas 2.6, 2.8]), and all objects are $\mathbb{P}$-fibrant. We write $\underline{\mathbb{M} M o d}(\underline{\mathcal{C}})$ (resp. $\underline{\mathbb{P M o d}}(\underline{\mathcal{C}})$ ) for the full $\mathcal{R}$-subcategory of $\underline{\mathbb{M o d}}(\underline{\mathcal{C}})$ consisting of $\mathbb{I}$-fibrant (resp. $\mathbb{P}$-cofibrant) objects.

Lemma 4.18. Let $\underline{\mathfrak{e}}$ be an $\mathcal{R}$-category. Then iCone maps $\mathbb{I}$-cofibrations (resp. $\mathbb{P}$ cofibrations) to trivial $\mathbb{I}$-cofibrations (resp. trivial $\mathbb{P}$-cofibrations). 
Proof. The $\mathbb{I}$-cofibrations are the monomorphisms; the $\mathbb{P}$-cofibrations are the monomorphisms with $\mathbb{P}$-cofibrant cokernel, as explained before [LS14, Lemma 2.4]. Now it is easy to adapt the proof of Lemma 3.9.

4.3.2. Projective and injective enhancements. Let $\underline{\mathcal{C}}$ be an $\mathcal{R}$-category. The derived category of $\underline{\mathcal{C}}$-modules is denoted $\mathrm{D}(\underline{\mathcal{C}})$. Its projective enhancement or $\mathbb{P}$-enhancement is the strongly pretriangulated (see [LS14, Lemma 2.4]) $\mathcal{R}$-category $\underline{\mathbb{P M} \operatorname{Mod}}(\underline{\mathrm{C}})$ together with the obvious R-linear triangulated equivalence

$$
[\underline{\mathrm{PM} O \mathrm{~d}}(\underline{\mathrm{C}})] \stackrel{\sim}{\rightarrow} \mathrm{D}(\underline{\mathrm{C}}) \text {. }
$$

Similarly, $\underline{\mathbb{M} \operatorname{Iod}}(\underline{\mathcal{C}})$ is the injective enhancement or $\mathbb{I}$-enhancement of $\mathrm{D}(\underline{\mathcal{C}})$.

\subsubsection{Enriched completeness.}

Lemma 4.19. Let $\underline{\mathrm{C}}$ be an $\mathcal{R}$-category. Then $\underline{\operatorname{Mod}}(\underline{\mathrm{C}})$ is tensored and cotensored, and $\mathcal{R}$-bicomplete.

Proof. Let $\underline{\mathcal{D}}$ be an $\mathcal{R}$-category. Let $M$ be a $\underline{\mathcal{C}}$-module, $L$ a $\underline{\mathcal{D}}$-module and $N$ a $\underline{\mathcal{C}} \otimes \underline{\mathcal{D}}$-module. Then there is an isomorphism

$$
\underline{\operatorname{Mod}}_{\underline{\mathcal{C}} \otimes \underline{\mathcal{D}}}(M \otimes L, N) \cong \underline{\operatorname{Mod}}_{\underline{\mathcal{C}}}\left(M, \operatorname{rHom}_{\underline{\mathcal{D}}}(L, N)\right)
$$

where $\operatorname{rHom}_{\underline{\mathcal{D}}}(L, N)$ is the $\underline{\mathcal{C}}$-module whose evaluation at $C \in \underline{\mathcal{C}}$ is $\underline{\mathbb{M o d}}_{\underline{\mathcal{D}}}(L, N(C,-))$. Similarly, there is an isomorphism

$$
\underline{\operatorname{Mod}}_{\underline{\mathcal{Q}} \otimes \underline{\mathcal{D}}}(M \otimes L, N) \cong \underline{\operatorname{Mod}}_{\underline{\mathcal{D}}}\left(L, \operatorname{IHom}_{\underline{\mathcal{e}}}(M, N)\right)
$$

where $\operatorname{IHom}_{\underline{\mathcal{e}}}(M, N)$ is the $\underline{\mathcal{D}}$-module whose evaluation at $D \in \underline{\mathcal{D}}$ is $\underline{\operatorname{Mod}}_{\underline{\mathcal{C}}}(M, N(-, D))$. Both rHom and IHom are $\mathcal{R}$-functors, and the above two isomorphisms are natural in $M, L$ and $N$.

Let $\underline{R}$ be the $\mathcal{R}$-category with one object whose endomorphisms are $\mathrm{R}$. Then $\mathbb{M o d}(\underline{R})=\mathrm{C}(\mathrm{R})=\mathcal{R}$ and $\underline{\operatorname{Mod}}(\underline{\mathrm{R}})=\underline{\mathrm{C}}(\mathrm{R})=\underline{\mathcal{R}}$. If we apply the above to $\underline{\mathcal{D}}=\underline{\mathrm{R}}$ then $\underline{\mathcal{C}} \otimes \underline{\mathrm{R}}=\underline{\mathcal{C}}$ and $\operatorname{IHom}_{\underline{\mathcal{e}}}(M, N)=\underline{\operatorname{Mod}}_{\underline{\mathfrak{e}}}(M, N)$ and we obtain isomorphisms

$$
\underline{\operatorname{Mod}}_{\underline{\mathcal{e}}}\left(M, \operatorname{rHom}_{\underline{R}}(R, N)\right) \cong \underline{\operatorname{Mod}}_{\underline{\mathcal{C}}}(M \otimes R, N) \cong \underline{\mathcal{R}}\left(R, \underline{\operatorname{Mod}}_{\underline{\mathcal{C}}}(M, N)\right)
$$

natural in $R \in \mathcal{R}, M, N \in \mathcal{M}$. They show that $\underline{\operatorname{Mod}}(\underline{\mathcal{C}})$ is tensored and cotensored (cf. (3.11), (3.12)). As a Grothendieck abelian category, $\mathbb{M o d}(\underline{\mathcal{C}})$ is bicomplete and hence $\underline{\operatorname{Mod}}(\underline{\mathcal{C}})$ is $\mathcal{R}$-bicomplete, by 3.2.5.

\subsubsection{Dg k-enriched functorial factorizations for modules over dg k-categories.}

Theorem 4.20. Let $\mathrm{k}$ be a field and $\underline{\mathrm{e}}$ a $\mathcal{U}$-small $\mathcal{K}$-category. Then the $\mathbb{I}$-model structure and the $\mathbb{P}$-model structure on $\mathbb{M o d}(\underline{\mathcal{C}})$ admit $\mathcal{K}$-enriched functorial factorizations. 
Proof. Obviously, $\underline{\mathbb{M o d}}(\underline{\mathcal{C}})$ is strongly pretriangulated. It is $\mathcal{K}$-bicomplete by Lemma 4.19. Both $\mathbb{I}$-model structure and $\mathbb{P}$-model structure are cofibrantly generated and their (trivial) cofibrations are stable under all shifts. Lemma 4.18 then shows that all assumptions of Theorem 3.6 are satisfied.

Remark 4.21. In the setting of Theorem 4.20, any $\mathcal{K}$-enriched $\mathbb{I}$-fibrant (resp. $\mathbb{P}$ cofibrant) replacement functor $\mathbf{i}: \underline{\mathbb{M o d}}(\underline{\mathcal{C}}) \rightarrow \underline{\mathbb{M} \operatorname{Mod}}(\underline{\mathcal{C}})(\operatorname{resp} \cdot \mathbf{p}: \underline{\mathbb{M o d}}(\underline{\mathcal{C}}) \rightarrow \underline{\operatorname{PMod}}(\underline{\mathcal{C}}))$ is a Drinfeld quotient of $\underline{\operatorname{Mod}}(\underline{\mathcal{C}})$ by its subcategory of acyclic objects. This is proved in the same way as Lemma 4.7 .

4.4. Ringed spaces. A $\mathcal{U}$-small ringed space is a pair $\left(X, \mathcal{O}_{X}\right)$ consisting of a $\mathcal{U}$ small topological space $X$ and a sheaf $\mathcal{O}_{X}$ of $\mathcal{U}$-small commutative rings on $X$. All ringed spaces and sites in the rest of this subsection are assumed to be $\mathcal{U}$-small. Any ringed space gives rise to a ringed site in the obvious way, and the categories of sheaves (of modules) on the ringed space and the associated ringed site coincide canonically. We adapt the notation from 4.2 in the obvious way to ringed spaces; for example we write $\mathrm{C}(X)$ for the category of complexes of $\mathcal{O}_{X}$-modules. Note that all the previous results about ringed sites apply to ringed spaces.

4.4.1. Flat model structure. Let $(X, \mathcal{O})$ be a ringed space. An object $F \in \mathrm{C}(X)$ is called $\mathbf{d g}$-flat if it is h-flat and all its components $F^{n}$ are flat $\mathcal{O}$-modules. This terminology coincides with that of [Gil07] by [Gil06, Prop. 5.6]. The flat model structure or $\mathbb{F}$-model structure on $\mathrm{C}(X)$ is given as follows (see [Gil07, Cor. 7.8]):

- the weak equivalences are the quasi-isomorphisms;

- the cofibrations are the monomorphisms with dg-flat cokernel.

This model structure turns $\mathrm{C}(X)$ into a cofibrantly generated model category. The $\mathbb{F}$ cofibrant objects are precisely the dg-flat objects of $\mathrm{C}(X)$. Let $\underline{\mathbb{F}}(X) \subset \underline{\mathrm{C}}(X)$ denote the full subcategory of $\mathbb{F}$-cofibrant objects.

Lemma 4.22. Let $(X, \mathcal{O})$ be a ringed space. Then iCone maps $\mathbb{F}$-cofibrations to trivial $\mathbb{F}$-cofibrations.

Proof. Apply Lemma 3.9.

\subsubsection{Ringed spaces over a ring.}

Definition 4.23. An R-ringed space or ringed space over $\mathrm{R}$ is a pair $\left(X, \mathcal{O}_{X}\right)$ consisting of a topological space $X$ and a sheaf $\mathcal{O}_{X}$ of commutative R-algebras on $X$. The category of R-ringed spaces is defined in the obvious way.

If we denote the topological space with a unique point by pt, then an R-ringed space can equivalently be defined as a ringed space over the ringed space (pt, R). Any $\mathrm{R}$-ringed space gives rise to an R-ringed site and to an R-ringed topos in the obvious way. In particular, all previous results for R-ringed sites can be applied to R-ringed 
spaces. For example, Lemma 4.15 shows that $\underline{\mathrm{C}}(\mathcal{X})$ is R-bicomplete if $(X, \mathcal{O})$ is an R-ringed space.

4.4.3. Dg k-enriched functorial factorizations for k-ringed spaces. Certainly Theorem 4.17 applies to ringed spaces over a field. There is a similar result for the flat model structure:

Theorem 4.24. Let $\left(X, \mathcal{O}_{X}\right)$ be a ringed space over a field $\mathrm{k}$. Then the $\mathbb{F}$-model structure on $\mathrm{C}(X)$ admits $\mathcal{K}$-enriched functorial factorizations.

Proof. Mutatis mutandis the proof of Theorem 4.17, using Lemma 4.22.

4.5. Modules over rings. All rings and modules in this subsection are assumed to be $\mathcal{U}$-small. If $A$ is a $\operatorname{ring}, \operatorname{Mod}(A)$ denotes the category of $A$-modules, and $\underline{A}$ denotes the $\mathrm{C}(\mathbb{Z})$-category with one object whose endomorphisms are $A$.

4.5.1. Model structures on complexes of modules. Let $A$ be a commutative ring. Note that $\underline{\operatorname{Mod}}(\underline{A})=\underline{\mathrm{C}}(\mathrm{pt}, A)=\underline{\mathrm{C}}(\operatorname{Mod}(A))=: \underline{\mathrm{C}}(A)$. We have introduced several model structures on $\operatorname{Mod}(\underline{A})=\mathrm{C}(\mathrm{pt}, A)=\mathrm{C}(A)$ whose weak equivalences are the quasi-isomorphisms. The $\mathbb{I}$-model structure on $\operatorname{Mod}(\underline{A})$ coincides with the $\mathbb{I}$-model structure on $\mathrm{C}(\mathrm{pt}, A)$ and the injective model structure on $\mathrm{C}(A)$ (because the cofibrations are the monomorphisms). The $\mathbb{P}$-model structure on $\mathbb{M o d}(\underline{A})$ coincides with the $\mathbb{E}$ model structure on $\mathrm{C}(\mathrm{pt}, A)$ (because the cofibrations can be generated by the same generating cofibrations).

4.5.2. Dg k-enriched functorial factorizations for modules over a k-algebra. Let $A$ be an algebra over a field $\mathrm{k}$. Then Theorem 4.20 can be applied to the $\mathcal{K}$-category $\underline{A}$ with one object whose endomorphisms are $A$; if $A$ is commutative, Theorems 4.17 and 4.24 can be applied to the k-ringed space (pt, $A$ ).

\subsubsection{The field case.}

Lemma 4.25. If $\mathrm{k}$ is a field, the following model structures on $\mathcal{K}=\mathbb{M} \operatorname{lod}(\underline{\mathrm{k}})=$ $\mathrm{C}(\mathrm{pt}, \mathrm{k})=\mathrm{C}(\mathrm{k})$ coincide: the $\mathbb{I}$-model structure on $\mathbb{M o d}(\underline{\mathrm{k}})$, the $\mathbb{I}$-model structure on $\mathrm{C}(\mathrm{pt}, \mathrm{k})$, the injective model structure on $\mathrm{C}(\mathrm{k})$, the $\mathbb{P}$-model structure on $\mathbb{M o d}(\underline{\mathrm{k}})$, the $\mathbb{E}$-model structure on $\mathrm{C}(\mathrm{pt}, \mathrm{k})$, the $\mathbb{F}$-model structure on $\mathrm{C}(\mathrm{pt}, \mathrm{k})$. All objects of $\mathcal{K}$ are fibrant and cofibrant with respect to any of these model structures.

Proof. We prove first that $\mathbb{I}$-model structure, $\mathbb{E}$-model structure and $\mathbb{F}$-model structure on $\mathrm{C}(\mathrm{pt}, \mathrm{k})$ coincide.

$\mathbb{I}=\mathbb{E}$ : By Lemma 4.10, any $\mathbb{E}$-cofibration is a monomorphism, i. e. an $\mathbb{I}$-cofibration. Conversely, Lemma 4.26 below shows that any monomorphism is an $\mathbb{E}$-cofibration: note that $0 \hookrightarrow[1] \mathrm{k}$ is a pushout of the $\mathbb{E}$-cofibration $\mathrm{k} \rightarrow \mathrm{iCone}(\mathrm{k})$. 
$\mathbb{I}=\mathbb{F}$ : The $\mathbb{F}$-cofibrations are the monomorphisms with dg-flat kernel. But any object of $\mathcal{K}$ is dg-flat: all vector spaces are flat, and any acyclic object of $\mathcal{K}$ is contractible, by Lemma 3.1 (or Lemma 4.27.(a) below).

The first claim of the lemma follows now from 4.5.1.

Trivially, all objects of $\mathcal{K}$ are $\mathbb{I}$-cofibrant. Recall that the $\mathbb{I}$-fibrant objects of $\mathcal{K}$ are the h-injective complexes with injective components. But all vector spaces are injective, and all objects of $\mathcal{K}$ are h-injective because any acyclic complex is contractible, by Lemma 3.1. Hence all objects of $\mathcal{K}$ are $\mathbb{I}$-fibrant. This proves the second claim.

Lemma 4.26. Let $\mathrm{k}$ be a field. Any monomorphism in $\mathcal{K}=\mathrm{C}(\mathrm{k})$ is isomorphic to a coproduct of shifts of the five monomorphisms $\mathrm{k} \stackrel{\mathrm{id}}{\rightarrow} \mathrm{k}$, iCone $(\mathrm{k}) \stackrel{\mathrm{id}}{\rightarrow} \mathrm{iCone}(\mathrm{k}), 0 \hookrightarrow \mathrm{k}$, $0 \hookrightarrow \mathrm{iCone}(\mathrm{k}), \mathrm{k} \stackrel{(\mathrm{id}, 0)^{\mathrm{t}}}{\longrightarrow} \mathrm{iCone}(\mathrm{k})$.

Proof. This follows from the slightly more general Lemma 4.27.(c) below.

\subsubsection{Complexes in a semisimple abelian category.}

Lemma 4.27. Let $\mathcal{A}$ be an abelian category which is semisimple in the sense that any short exact sequence splits. Then:

(a) Any object $A \in \mathrm{C}(\mathcal{A})$ is isomorphic to $S \oplus \mathrm{iCone}(T)$ for objects $S, T \in \mathcal{A}^{\mathbb{Z}}$; more precisely, $S \cong H(A)$ and $T \cong B(A)$ (where $B(A)$ denotes the boundaries).

(b) All objects iCone $(T)$ for $T \in \mathcal{A}^{\mathbb{Z}}$ are projective and injective in $\mathrm{C}(\mathcal{A})$;

(c) Any short exact sequence in $\mathrm{C}(\mathcal{A})$ is isomorphic to a direct sum of short exact sequences of the following five types: $S \stackrel{\mathrm{id}}{\hookrightarrow} S \rightarrow 0$, iCone $(T) \stackrel{\mathrm{id}}{\rightarrow} \mathrm{iCone}(T) \rightarrow 0$, $0 \hookrightarrow U \stackrel{\text { id }}{\rightarrow} U, 0 \hookrightarrow \mathrm{iCone}(V) \stackrel{\text { id }}{\rightarrow} \mathrm{iCone}(V), W \stackrel{(\mathrm{id}, 0)^{\mathrm{t}}}{\rightarrow} \mathrm{iCone}(W) \stackrel{(0, \mathrm{id})}{\longrightarrow}[1] W$, where $S, T, U, V, W$ are objects of $\mathcal{A}^{\mathbb{Z}}$.

Proof. (a) Consider the short exact sequences $Z(A) \hookrightarrow A \stackrel{d}{\rightarrow}[1] B(A)$ and $B(A) \hookrightarrow$ $Z(A) \rightarrow H(A)$ in $\mathrm{C}(\mathcal{A})$. All terms in these sequences except for $A$ have vanishing differential. Since $\mathcal{A}$ is semisimple, the second sequence splits and hence the first sequence is isomorphic to $H(A) \oplus B(A) \hookrightarrow A \stackrel{d}{\rightarrow}[1] B(A)$. This sequence splits in $\mathcal{A}^{\mathbb{Z}}$ (i. e. if we forget the differentials) and we obtain $A \cong H(A) \oplus \operatorname{iCone}(B(A))$ in $\mathrm{C}(\mathcal{A})$.

(b) The exact functor "forget the differential" $\mathrm{C}(\mathcal{A}) \rightarrow \mathcal{A}^{\mathbb{Z}}$ has $T \mapsto \mathrm{iCone}(T)$ as a right adjoint and $T \mapsto[-1] \mathrm{iCone}(T)$ as a left adjoint. Hence iCone: $\mathcal{A}^{\mathbb{Z}} \rightarrow \mathrm{C}(\mathcal{A})$ preserves injective (resp. projective) objects. Since $\mathcal{A}$ is semisimple, all objects of $\mathcal{A}^{\mathbb{Z}}$ are injective and projective.

(c) Let $E \hookrightarrow F \rightarrow G$ be a short exact sequence in $\mathrm{C}(\mathcal{A})$. By (a) and (b), our sequence is isomorphic to the direct sum of the three short exact sequences $\mathrm{iCone}(T) \stackrel{\text { id }}{\hookrightarrow} \mathrm{iCone}(T) \rightarrow 0,0 \hookrightarrow \mathrm{iCone}(V) \stackrel{\text { id }}{\rightarrow} \mathrm{iCone}(V)$, and $E^{\prime} \hookrightarrow F^{\prime} \rightarrow G^{\prime}$ where $T=B(E), V=B(G), E^{\prime}=H(E)$, and $G^{\prime}=H(G)$ are objects of $\mathcal{A}^{\mathbb{Z}}$ and $F^{\prime}$ is a 
suitable object of $\mathrm{C}(\mathcal{A})$. In $\mathcal{A}^{\mathbb{Z}}$ our sequence $E^{\prime} \hookrightarrow F^{\prime} \rightarrow G^{\prime}$ is isomorphic to the obvious short exact sequence $E^{\prime} \hookrightarrow E^{\prime} \oplus G^{\prime} \rightarrow G^{\prime}$, and the differential $d_{F^{\prime}}$ of $F^{\prime}$ maps $E^{\prime}$ to zero and $G^{\prime}$ to $E^{\prime}$. Let $W$ denote the image of $d_{F^{\prime}}$. By semisimplicity we can decompose $E^{\prime} \cong S \oplus W$ and $G^{\prime} \cong[1] W \oplus U$ such that $d_{F^{\prime}}^{n}$ is given by the composition (4.17)

$F^{\prime n} \cong S^{n} \oplus W^{n} \oplus W^{n+1} \oplus U^{n} \stackrel{\text { proj. }}{\longrightarrow} W^{n+1} \stackrel{\text { incl. }}{\longrightarrow} S^{n+1} \oplus W^{n+1} \oplus W^{n+2} \oplus U^{n+1} \cong F^{\prime n+1}$.

The claim follows.

4.6. Dg k-enriched model categories. This subsection may be skipped on a first reading. Our aim is to show Theorem 4.30 for later use in 9. All model categories in this subsection are to be considered with respect to the universe $\mathcal{U}$.

Let $\mathrm{k}$ be a field and $\underline{\mathcal{K}}=\underline{\mathrm{C}}(\mathrm{k})$. We view $\mathcal{K}$ as a model category equipped with any of the identical model structures of Lemma 4.25. The following result is similar to Lemma 3.7.

Lemma 4.28. Let $\mathrm{k}$ be a field. Let $\underline{\mathcal{M}}$ be a strongly pretriangulated tensored $\mathcal{K}$ category, with tensored structure given by the bifunctor $\odot$. Assume that the underlying category $\mathcal{M}$ is equipped with a model structure turning it into a model category such that

(a) the set of cofibrations and the set of trivial cofibrations are stable under all shifts;

(b) the functor iCone(-) maps cofibrations to trivial cofibrations;

(c) for each cofibration $E \rightarrow F$ the morphism

$$
\mathrm{iCone}(E) \bigsqcup_{E} F \rightarrow \mathrm{iCone}(F)
$$

(cf. (3.16)) is a cofibration.

Then the following is true: if $i: E \rightarrow F$ is a cofibration in $\mathcal{M}$ and $j: K \rightarrow L$ is a cofibration in $\mathcal{K}$, then the pushout-product morphism

$$
i \odot j: E \odot L \bigsqcup_{E \odot K} F \odot K \rightarrow F \odot L
$$

induced by the commutative diagram

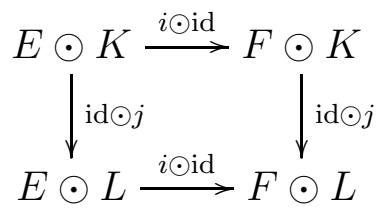

is a cofibration which is trivial if $i$ or $j$ is a trivial cofibration.

Proof. Lemma 4.26 allows us to write $j$ as a coproduct of shifts of monomorphisms $\mathrm{k} \stackrel{\text { id }}{\rightarrow} \mathrm{k}, \mathrm{iCone}(\mathrm{k}) \stackrel{\text { id }}{\rightarrow} \mathrm{iCone}(\mathrm{k}), 0 \hookrightarrow \mathrm{k}, 0 \hookrightarrow \mathrm{iCone}(\mathrm{k}), \mathrm{k} \stackrel{(\mathrm{id}, 0)^{\mathrm{t}}}{\rightarrow} \mathrm{iCone}(\mathrm{k})$. As in the 
proof of Lemma 3.7 we see that it is enough to consider these basic five monomorphisms. Let $i: E \rightarrow F$ be a cofibration.

If $j$ is an isomorphism, then $i \odot j$ is an isomorphism and hence a trivial cofibration.

If $j$ is $0 \hookrightarrow \mathrm{k}$, then $i \odot j$ is isomorphic to $i$. This uses $M \odot 0 \cong 0$ and $(-\odot \mathrm{k}) \cong \mathrm{id}$.

If $j$ is the trivial cofibration $0 \hookrightarrow \mathrm{iCone}(\mathrm{k})$, then $i \odot j$ is isomorphic to the morphism $\mathrm{iCone}(i): \mathrm{iCone}(E) \rightarrow \mathrm{iCone}(F)$ which is a trivial cofibration by assumption. This uses $M \odot \mathrm{iCone}(\mathrm{k}) \cong \mathrm{iCone}(M)$ naturally in $M$, and $M \odot 0 \cong 0$.

Now assume that $j$ is the cofibration $\mathrm{k} \stackrel{(\mathrm{id}, 0)^{\mathrm{t}}}{\longrightarrow} \mathrm{iCone}(\mathrm{k})$. Note that the functor $M \mapsto(M \odot \mathrm{k} \rightarrow M \odot \mathrm{iCone}(\mathrm{k}))$ is isomorphic to $M \mapsto(M \rightarrow \mathrm{iCone}(M))$. Therefore $i \hat{\odot} j$ is isomorphic to the morphism (4.18) and therefore a cofibration. If $i: E \rightarrow F$ is a trivial cofibration, then the composition

$$
\mathrm{iCone}(E) \rightarrow \mathrm{iCone}(E) \bigsqcup_{E} F \rightarrow \mathrm{iCone}(F)
$$

is a trivial cofibration by assumption and so is the arrow on the left as a pushout of $i$. Therefore the cofibration on the right is trivial by the 2-out-of-3 property.

Proposition 4.29. Let $\mathrm{k}$ be a field.

(a) The model category $\mathcal{K}$ is monoidal and excellent (see [Lur09, Def. A.3.1.2, A.3.2.16]).

(b) Additionally to the assumptions of Lemma 4.28, assume that $\underline{\mathcal{N}}$ is cotensored over $\mathcal{K}$. Then $\underline{\mathcal{N}}$ is a $\mathcal{K}$-enriched model category (see [Lur09, Def. A.3.1.5]).

Proof. (a) Lemma 4.28 (using Lemma 3.9 and Corollary 3.10) and Lemma 4.25 show that $\mathcal{K}$ is a monoidal model category. Certainly, $\mathcal{K}$ is a combinatorial model category (cf. the proof of Theorem 4.30 below), and the set of quasi-isomorphisms is stable under $\mathcal{U}$-small filtered colimits: this is true for the category of complexes in any abelian category satisfying Grothendieck's condition AB5. Now use [Law16, Thm. 1] to see that $\mathcal{K}$ is excellent.

Claim (b) follows from Lemma 4.28.

Theorem 4.30. Let $\mathrm{k}$ be a field. The following $\mathcal{K}$-categories are combinatorial (see [Lur09, Def. A.2.6.1]) $\mathcal{K}$-enriched model categories:

(a) $\underline{\mathrm{C}}(\mathcal{X})$ with $\mathrm{C}(\mathcal{X})$ carrying the $\mathbb{I}$ - or $\mathbb{E}$-model structure, for a $\mathcal{U}$-small $\mathrm{k}$-ringed site $(\mathcal{X}, \mathcal{O})$;

(b) $\underline{\operatorname{Mod}}(\underline{\mathrm{C}})$ with $\operatorname{Mod}(\underline{\mathrm{C}})$ carrying the $\mathbb{I}$ - or $\mathbb{P}$-model structure, for a $\mathcal{U}$-small $\mathcal{K}$ category $\underline{\mathrm{e}}$;

(c) $\underline{\mathrm{C}}(X)$ with $\mathrm{C}(X)$ carrying the $\mathbb{F}$ - or $\mathbb{I}$ - or $\mathbb{E}$-model structure, for a $\mathcal{U}$-small k-ringed space $(X, \mathcal{O})$;

(d) $\underline{\mathrm{C}}(A)$ with $\mathrm{C}(A)$ carrying any of the model structures from 4.5.1 where $A$ is a U-small commutative $\mathrm{k}$-algebra. 
Proof. All model categories considered are cofibrantly generated; they are combinatorial because any Grothendieck abelian category is locally presentable, by [Bek00, Prop. 3.10], and hence presentable in Lurie's terminology. They are $\mathcal{K}$-enriched model categories because Proposition 4.29.(b) is applicable: use the proven results of section 4, and Corollary 3.10 (mutatis mutandis for $\operatorname{Mod}(\underline{\mathrm{e}})$ ).

Remark 4.31. Theorem 4.30 and Proposition 4.29.(a) show that [Lur09, Prop. A.3.3.2] is applicable to all the combinatorial $\mathcal{K}$-enriched model categories mentioned in Theorem 4.30: the category of $\mathcal{K}$-functors from a $\mathcal{U}$-small $\mathcal{K}$-category to one of these $\mathcal{K}$-categories carries the projective and the injective combinatorial model structure, each of them turning it into a model category. This will be used in 9 .

\section{Some 2-Multichtegories}

The main goal of this section is to introduce the R-linear 2-multicategory $\mathrm{ENH}_{\mathrm{R}}$ of enhancements. This is a key definition which allows a concise formulation of our lifting results later on. We also introduce a multicategory of formulas.

For the rest of this article, we fix universes $\mathcal{U} \in \mathcal{V}$.

By an R-category we mean an R-category with $\mathcal{V}$-small Hom-sets. By a $\mathcal{V}$-small $\mathcal{R}$-category we mean a $\mathcal{V}$-small $\mathcal{R}_{\mathcal{V}}$-category (i. e. the set of objects is $\mathcal{V}$-small and the Hom-sets are $\mathcal{V}$-small dg R-modules).

5.1. 2-multicategories of categories, dg categories, and triangulated categories. Our reference for enriched multicategories is [Lei04]. Recall that a 2multicategory (with $\mathcal{V}$-small Hom-categories) is a multicategory enriched in the symmetric monoidal category of $(\mathcal{V}$-small) categories. By an R-linear 2-multicategory we mean a 2-multicategory enriched in the symmetric monoidal category of $\mathcal{V}$-small R-categories, cf. [GK08, Def. 2.5]. In such a multicategory the set of all 2-morphisms with same source and target is a $\mathcal{V}$-small R-module.

5.1.1. Categories and $d g$ categories. The monoidal category of $\mathcal{V}$-small R-categories gives rise to the multicategory $\mathrm{cat}_{\mathrm{R}}$, by [Lei04, Ex. 2.1.3]. Its objects are the $\mathcal{V}$-small R-categories, and its morphism sets

$$
\operatorname{cat}_{\mathrm{R}}\left(\mathcal{C}_{1}, \ldots, \mathcal{C}_{n} ; \mathcal{D}\right)
$$

are R-functors $\mathcal{C}_{1} \otimes \cdots \otimes \mathcal{C}_{n} \rightarrow \mathcal{D}$, or, equivalently, R-multilinear functors $\mathcal{C}_{1} \times \cdots \times \mathcal{C}_{n} \rightarrow$ $\mathcal{D}$. We adopt this latter viewpoint because later on we will focus our attention on triangulated R-categories. Note that $\operatorname{cat}_{\mathrm{R}}(\emptyset ; \mathcal{D})=\operatorname{Obj} \mathcal{D}$ where $\emptyset$ is the empty sequence because the empty tensor product of R-categories is the R-category with one object whose endomorphisms are R.

The multicategory cat $t_{R}$ is the underlying multicategory of the R-linear 2-multicategory $\mathrm{CAT}_{\mathrm{R}}$ of $\mathcal{V}$-small R-categories whose objects are the $\mathcal{V}$-small R-categories and 
whose morphism R-categories

$$
\operatorname{CAT}_{\mathrm{R}}\left(\mathcal{C}_{1}, \ldots, \mathcal{C}_{n} ; \mathcal{D}\right)
$$

are the categories of R-multilinear functors $F, G: \mathcal{C}_{1} \times \cdots \times \mathcal{C}_{n} \rightarrow \mathcal{D}$ and R-natural transformations $\sigma, \tau: F \rightarrow G$ (note that these morphism categories (or Hom-categories) are indeed $\mathcal{V}$-small by the obvious variant of Remark 3.2.(a)). Since $\mathcal{D}$ is an Rcategory, $r \sigma+r^{\prime} \tau$ is again an R-natural transformation $F \rightarrow G$, for $r, r^{\prime} \in \mathrm{R}$; this explains that $\mathrm{CAT}_{\mathrm{R}}$ is R-linear. Note that $\operatorname{CAT}_{\mathrm{R}}(\emptyset ; \mathcal{D})=\mathcal{D}$.

The R-linear 2-multicategory DGCAT $\mathrm{DG}_{\mathrm{R}}$ of $\mathcal{V}$-small $\mathcal{R}$-categories (= dg R-categories) is defined similarly: its objects are the $\mathcal{V}$-small $\mathcal{R}$-categories, and its morphism $\mathrm{R}$ categories

$$
\operatorname{DGCAT}_{\mathrm{R}}\left(\underline{\mathcal{A}}_{1}, \ldots, \underline{\mathcal{A}}_{n} ; \underline{\mathcal{B}}\right)
$$

are the $\mathcal{V}$-small R-categories of $\mathcal{R}$-functors $\underline{\mathcal{A}}_{1} \otimes \cdots \otimes \underline{\mathcal{A}}_{n} \rightarrow \underline{\mathcal{B}}$ and $\mathcal{R}$-natural transformations. The underlying multicategory of $\mathrm{DGCAT}_{\mathrm{R}}$ is the multicategory dgcat $_{\mathrm{R}}$ of $\mathcal{R}$-categories and $\mathcal{R}$-functors. Note that $\operatorname{DGCAT}_{\mathrm{R}}(\emptyset ; \underline{\mathcal{B}})=\mathcal{B}$.

Mapping a $\mathcal{V}$-small $\mathcal{R}$-category $\underline{\mathcal{A}}$ to its homotopy category $[\underline{\mathcal{A}}]$ induces a functor

$$
[-]: \mathrm{DGCAT}_{\mathrm{R}} \rightarrow \mathrm{CAT}_{\mathrm{R}}
$$

of R-linear 2-multicategories: a 1-morphism $F: \underline{\mathcal{A}}_{1} \otimes \cdots \otimes \underline{\mathcal{A}}_{n} \rightarrow \underline{\mathcal{B}}$ is mapped to the composition

$$
[F]:\left[\underline{\mathcal{A}}_{1}\right] \times \cdots \times\left[\underline{\mathcal{A}}_{n}\right] \rightarrow\left[\underline{\mathcal{A}}_{1} \otimes \cdots \otimes \underline{\mathcal{A}}_{n}\right] \stackrel{[F]}{\longrightarrow}[\underline{\mathcal{B}}],
$$

again denoted $[F]$, and a 2-morphism $\tau: F \rightarrow G$ is mapped to the induced 2-morphism $[\tau]:[F] \rightarrow[G]$.

5.1.2. Triangulated categories and pretriangulated $d g$ categories. We denote the Rlinear 2-multicategory of $\mathcal{V}$-small triangulated R-categories by $\mathrm{TRCAT}_{\mathrm{R}}$. Its objects are $\mathcal{V}$-small triangulated R-categories, and its morphism R-categories

$$
\operatorname{TRCAT}_{\mathrm{R}}\left(\mathcal{T}_{1}, \ldots, \mathcal{T}_{n} ; \mathcal{S}\right)
$$

are the R-categories of triangulated R-multilinear functors $\mathcal{T}_{1} \times \cdots \times \mathcal{T}_{n} \rightarrow \mathcal{S}$ (i. e. R-multilinear functors of R-categories with translation that send triangles in each argument to triangles, cf. [KS06, Def. 10.3.6]) and their transformations. Since $\mathcal{S}$ is additive, these R-categories are additive. We use the convention $\operatorname{TRCAT}_{\mathrm{R}}(\emptyset ; \mathcal{S})=\mathcal{S}$. The underlying multicategory of $\mathrm{TRCAT}_{\mathrm{R}}$ is the multicategory trcat $_{\mathrm{R}}$ of $\mathcal{V}$-small triangulated R-categories and triangulated R-functors.

If $\underline{\mathcal{A}}_{1}, \ldots, \underline{\mathcal{A}}_{n}, \underline{\mathcal{B}}$ are $\mathcal{V}$-small pretriangulated $\mathcal{R}$-categories and $F: \underline{\mathcal{A}}_{1} \otimes \cdots \otimes \underline{\mathcal{A}}_{n} \rightarrow \underline{\mathcal{B}}$ is an $\mathcal{R}$-functor, then $[F]:\left[\underline{\mathcal{A}}_{1}\right] \times \cdots \times\left[\underline{\mathcal{A}}_{n}\right] \rightarrow[\underline{\mathcal{B}}]$ is a triangulated $\mathcal{R}$-multilinear functor (by the obvious generalization of Remark 3.4); if $G$ is another such $\mathcal{R}$-functor and $\tau: F \rightarrow G$ is an $\mathcal{R}$-natural transformation, then $[\tau]$ is a transformation of triangulated R-multilinear functors. 
Let DGCAT $\mathrm{R}_{\mathrm{R}}^{\mathrm{pre} \text {-tr }}$ be the full R-linear 2-multisubcategory of $\mathrm{DGCAT}_{\mathrm{R}}$ whose objects are the pretriangulated $\mathcal{R}$-categories. The above discussion shows that the functor (5.4) induces a functor

$$
[-]: \text { DGCAT }_{\mathrm{R}}^{\mathrm{pre}-\mathrm{tr}} \rightarrow \mathrm{TRCAT}_{\mathrm{R}}
$$

of R-linear 2-multicategories so that we obtain a commutative diagram

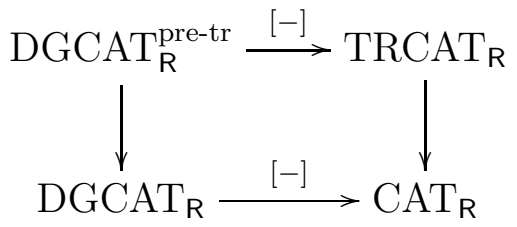

of R-linear 2-multicategories.

Remark 5.1. The vertical functor on the right in (5.8) trivially reflects 1-isomorphisms and 2-isomorphisms.

\subsubsection{Objectwise homotopy equivalences.}

Definition 5.2. Let $\underline{\mathcal{A}}_{1}, \ldots, \underline{\mathcal{A}}_{n}, \underline{\mathcal{B}} \in \mathrm{DGCAT}_{\mathrm{R}}$. We call a morphism $\tau: F \rightarrow G$ in $\operatorname{DGCAT}_{\mathrm{R}}\left(\underline{\mathcal{A}}_{1}, \ldots, \underline{\mathcal{A}}_{n} ; \underline{\mathcal{B}}\right)$ an objectwise homotopy equivalence if

$$
\tau_{\left(A_{1}, \ldots, A_{n}\right)}: F\left(A_{1}, \ldots, A_{n}\right) \rightarrow G\left(A_{1}, \ldots, A_{n}\right)
$$

is a homotopy equivalence in $\underline{\mathcal{B}}$, for all $\left(A_{1}, \ldots, A_{n}\right) \in \underline{\mathcal{A}}_{1} \otimes \cdots \otimes \underline{\mathcal{A}}_{n}$. Our notation for an objectwise homotopy equivalence is $F \stackrel{[\sim]}{\longrightarrow} G$.

Equivalently, an objectwise homotopy equivalence is a 2-morphism in $\operatorname{DGCAT}_{R}$ whose image under the functor (5.4) is a 2-isomorphism.

Remark 5.3. The vertical (resp. horizontal) composition in $\mathrm{DGCAT}_{\mathrm{k}}$ of objectwise homotopy equivalences is an objectwise homotopy equivalence.

\subsubsection{Existence of additive linear localizations.}

Definition 5.4 (cf. [Hir03, Def. 8.3.1]). An additive R-localization of an additive R-category $\mathcal{M}$ with respect to some subset $\mathcal{W}$ of the set $\operatorname{Mor}(\mathcal{M})$ of morphisms in $\mathcal{M}$ is an additive R-category L $\mathcal{M}$ together with an R-functor $\gamma: \mathcal{M} \rightarrow \mathrm{L} \mathcal{M}$ (which is automatically additive) that maps all morphisms in $\mathcal{W}$ to isomorphisms and is universal with this property: given any R-functor $\varphi: \mathcal{M} \rightarrow \mathcal{N}$ to an additive Rcategory $\mathcal{N}$ mapping all morphisms in $\mathcal{W}$ to isomorphisms there is a unique R-functor $\delta: \operatorname{LM} \rightarrow \mathcal{N}$ such that $\varphi=\delta \gamma$.

Proposition 5.5. Let $\mathcal{V}$ be a universe, $\mathcal{M}$ a $\mathcal{V}$-small additive $\mathrm{R}$-category, and $\mathcal{W} \subset$ Mor $\mathcal{M}$ a subset. Assume that $\mathcal{W}$ is closed under direct sums, i. e. for all morphisms $w: A \rightarrow B$ und $w^{\prime}: A^{\prime} \rightarrow B^{\prime}$ in $\mathcal{W}$ the morphism $w \oplus w^{\prime}: A \oplus A^{\prime} \rightarrow B \oplus B^{\prime}$ is again in $\mathcal{W}$. Then there is a an additive $\mathrm{R}$-localization $\gamma: \mathcal{M} \rightarrow \mathrm{L} \mathcal{M}$ of $\mathcal{M}$ with respect to $\mathcal{W}$ 
where $\mathrm{L} \mathcal{M}$ is $\mathcal{V}$-small. Moreover, the underlying functor is the ordinary localization of the category $\mathcal{M}$ with respect to $\mathcal{W}$.

Proof. The existence of the localization $\gamma: \mathcal{M} \rightarrow \mathrm{L} \mathcal{M}$ as an ordinary category and the fact that $\mathrm{L} \mathcal{M}$ is $\mathcal{V}$-small are well-known, see e.g. [GR17, Thm. 1.6.9 together with its proof]. Then [Cis10] shows that $\mathrm{L} \mathcal{M}$ is additive and that $\gamma$ is an additive functor. Now it is clear how to multiply a morphism $A \rightarrow B$ in $\mathrm{L} \mathcal{M}$ by an element $r \in R$ : just precompose with $\gamma\left(\operatorname{rid}_{A}\right)$ (or postcompose with $\gamma\left(\operatorname{rid}_{B}\right)$ ). All claims of the proposition follow.

Corollary 5.6. Let $\underline{\mathcal{A}}, \underline{\mathcal{B}}$ be $\mathcal{V}$-small $\mathcal{R}$-categories, and assume that $\underline{\mathcal{B}}$ is additive. Then the additive $\mathrm{R}$-localization of $\operatorname{DGCAT}_{\mathrm{R}}(\underline{\mathcal{A}}, \underline{\mathcal{B}})$ with respect to the set of objectwise homotopy equivalences exists and is $\mathcal{V}$-small.

Proof. Since $\underline{\mathcal{B}}$ is additive, the $\mathcal{V}$-small R-category $\operatorname{DGCAT}_{\mathrm{R}}(\underline{\mathcal{A}}, \underline{\mathcal{B}})$ is additive. The direct sum of objectwise homotopy equivalences is again an objectwise homotopy equivalence. Hence we can use Proposition 5.5.

We can and will assume in the following that all considered additive R-localization functors are the identity maps on the sets of objects.

5.2. The 2-multicategory of $\mathbf{d g}$ R-enhancements. Let $\widetilde{\mathrm{ENH}}_{\mathrm{R}}$ be the full R-linear 2-multisubcategory of $\mathrm{DGCAT}_{\mathrm{R}}$ consisting of all additive pretriangulated $\mathcal{V}$-small $\mathcal{R}$ categories. Any strongly pretriangulated $\mathcal{V}$-small $\mathcal{R}$-category is additive and hence an object of $\widetilde{\mathrm{ENH}}_{\mathrm{R}}$. The R-linear 2-multicategory $\mathrm{ENH}_{\mathrm{R}}$ of $\mathcal{R}$-enhancements is defined as follows. Its objects are the objects of $\widehat{\mathrm{ENH}}_{\mathrm{R}}$. Given objects $\underline{\mathcal{A}}_{1}, \ldots, \underline{\mathcal{A}}_{n}$ and $\underline{\mathcal{B}}$ of $\mathrm{ENH}_{\mathrm{R}}$, the R-category of morphisms

$$
\mathrm{ENH}_{\mathrm{R}}\left(\underline{\mathcal{A}}_{1}, \ldots, \underline{\mathcal{A}}_{n} ; \underline{\mathcal{B}}\right)
$$

is defined to be the target of the additive R-localization (see Definition 5.4) of the $\mathcal{V}$-small additive $\mathrm{R}$-category

$$
\widetilde{\operatorname{ENH}}_{\mathrm{R}}\left(\underline{\mathcal{A}}_{1}, \ldots, \underline{\mathcal{A}}_{n} ; \underline{\mathcal{B}}\right)=\operatorname{DGCAT}_{\mathrm{R}}\left(\underline{\mathcal{A}}_{1} \otimes \cdots \otimes \underline{\mathcal{A}}_{n}, \underline{\mathcal{B}}\right)
$$

with respect to the set of objectwise homotopy equivalences. It exists and is a $\mathcal{V}$ small additive R-category, by Corollary 5.6. The definition of the identities in $\mathrm{ENH}_{\mathrm{R}}$ is obvious, and compositions are defined in the obvious way using Remark 5.3 and the fact that additive R-localization of additive R-categories commutes with finite products.

Remark 5.7. Each object $\underline{B}$ of $\mathrm{ENH}_{\mathrm{R}}\left(\right.$ or $\widetilde{\mathrm{ENH}}_{\mathrm{R}}$ ) is a pretriangulated $\mathcal{R}$-category, so $[\underline{\mathcal{B}}]$ is a triangulated R-category and $\underline{\mathcal{B}}$ together with the identity functor $[\underline{\mathcal{B}}] \rightarrow[\underline{\mathcal{B}}]$ is an $\mathcal{R}$-enhancement of $[\underline{\mathcal{B}}]$, see Definition 3.5. This explains why $\mathrm{ENH}_{\mathrm{R}}$ is called the 2-category of enhancements. For the purpose of this article, the most important objects of $\mathrm{ENH}_{\mathrm{R}}$ are the strongly pretriangulated $\mathcal{R}$-categories $\underline{\mathbb{I}}(\mathcal{X})$, where $(\mathcal{X}, \mathcal{O})$ 
is a $\mathcal{U}$-small R-ringed site. Then $[\underline{\mathbb{I}}(\mathcal{X})] \stackrel{(4.1)}{\sim} \mathrm{D}(\mathcal{X})$ is an equivalence and $\mathbb{I}(\mathcal{X})$ is usually viewed as an $\mathcal{R}$-enhancement of $\mathrm{D}(\mathcal{X})$. Similarly, $\underline{\mathbb{M} \operatorname{lod}}(\underline{\mathcal{C}})$ and $\underline{\mathbb{P M} \mathbb{M}}(\underline{\mathcal{C}})$ are important objects of $\mathrm{ENH}_{\mathrm{R}}$, if $\underline{\mathcal{C}}$ is a $\mathcal{U}$-small $\mathcal{R}$-category. They are enhancements of $\mathrm{D}(\underline{\mathcal{C}})$, cf. (4.13). Note that $\underline{\mathbb{I}}(\mathcal{X}), \underline{\mathbb{M} \operatorname{Mod}}(\underline{\mathcal{C}})$ and $\underline{\mathbb{P M o d}}(\underline{\mathcal{C}})$ have $\mathcal{U}$-small Hom-sets and objects in $\mathcal{U}$, by the discussion in 4.2 and 4.3.1, so they are in particular $\mathcal{V}$-small.

Remark 5.8. If $\underline{\mathcal{B}}$ is any strongly pretriangulated $\mathcal{V}$-small $\mathcal{R}$-category, it is straightforward to see that $\mathcal{B} \rightarrow[\underline{\mathcal{B}}]$ is the additive $\mathrm{R}$-localization of $\mathcal{B}$ with respect to the set of homotopy equivalences. In particular, $\operatorname{ENH}_{\mathrm{R}}(\emptyset, \underline{\mathcal{B}})=[\underline{\mathcal{B}}]$ as an R-category because it is defined as the additive R-localization of $\widetilde{\operatorname{ENH}}_{\mathrm{R}}(\emptyset, \underline{\mathcal{B}})=\operatorname{DGCAT}_{\mathrm{R}}(\emptyset, \underline{\mathcal{B}})=\mathcal{B}$ with respect to the set of (objectwise) homotopy equivalences. In particular, objects and morphisms in $\mathcal{B}$ give rise to 1-morphisms and 2-morphisms in $\mathrm{ENH}_{\mathrm{R}}$.

The construction of $\mathrm{ENH}_{\mathrm{R}}$ yields a functor

$$
\delta: \widetilde{\mathrm{ENH}}_{\mathrm{R}} \rightarrow \mathrm{ENH}_{\mathrm{R}}
$$

of R-linear 2-multicategories. We can and will assume that $\delta$ is the identity on 1morphisms. By the universal property of additive R-localizations (and Remark 5.1) there is a unique functor

$$
[-]: \mathrm{ENH}_{\mathrm{R}} \rightarrow \mathrm{TRCAT}_{\mathrm{R}}
$$

of R-linear 2-multicategories such that the diagram

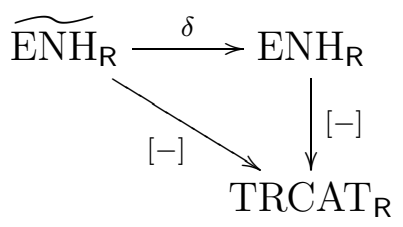

of R-linear 2-multicategories commutes where the diagonal arrow is obtained from (5.7).

Let $\mathrm{enh}_{\mathrm{R}}$ be the underlying multicategory of $\widetilde{\mathrm{ENH}}_{\mathrm{R}}$. It coincides with the underlying multicategory of $\mathrm{ENH}_{\mathrm{R}}$ and comes with the obvious functor

$$
[-]: \text { enh }_{\mathrm{R}} \rightarrow \text { trcat }_{\mathrm{R}}
$$

Remark 5.9. Let

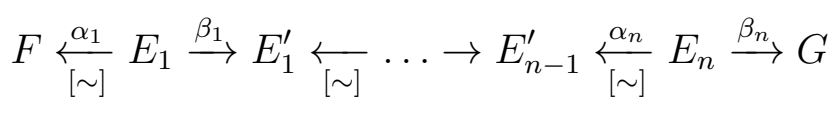

be a zig-zag of 2-morphisms in $\widetilde{\mathrm{ENH}}_{\mathrm{R}}$ where all morphisms $\alpha_{i}$ are objectwise homotopy equivalences. Then all $\delta\left(\alpha_{i}\right)$ are invertible in $\mathrm{ENH}_{\mathrm{R}}$ and

$$
\delta\left(\beta_{n}\right) \delta\left(\alpha_{n}\right)^{-1} \cdots \delta\left(\beta_{1}\right) \delta\left(\alpha_{1}\right)^{-1}: \delta(F) \rightarrow \delta(G)
$$

defines a 2-morphism $F \rightarrow G$ in $\mathrm{ENH}_{\mathrm{R}}$; this 2-morphism is a 2-isomorphism if all $\beta_{i}$ are objectwise homotopy equivalences. 
5.3. Some useful equalities. For later use we prove two additional results. We now work over the field $\mathrm{k}$. In this subsection let $\underline{\mathcal{N}}$ be either

(a) $\underline{\mathrm{C}}(\mathcal{X})$ with $\mathrm{C}(\mathcal{X})$ carrying the $\mathbb{I}$-model structure, for a $\mathcal{U}$-small k-ringed site $(\mathcal{X}, \mathcal{O})$, or

(b) $\underline{\operatorname{Mod}}(\underline{\mathcal{C}})$ with $\mathbb{M o d}(\underline{\mathcal{C}})$ carrying the $\mathbb{I}$-model structure, for a $\mathcal{U}$-small $\mathcal{K}$-category $\underline{\mathrm{e}}$.

We remind the reader that all objects of $\mathcal{N}$ are $\mathbb{I}$-cofibrant and hence the set of $\mathbb{I}$ fibrant objects in $\underline{\mathcal{M}}$ coincides with the set of $\mathbb{I}$-bifibrant objects. Let $\underline{\mathcal{M}}_{\text {bifib }}$ be the full subcategory of $\underline{\mathcal{M}}$ of $\mathbb{I}$-fibrant objects. So either $\underline{\mathcal{M}}_{\text {bifib }}=\underline{\mathbb{I}}(\mathcal{X})$ or $\underline{\mathcal{M}}_{\mathrm{bifib}}=\underline{\mathbb{M}} \underline{\mathbb{M}}(\underline{\mathcal{C}})$.

Proposition 5.10. Let $\underline{\mathcal{N}}$ be as above. Let $\underline{\mathcal{B}}$ be a full additive $\mathcal{K}$-subcategory of $\underline{\mathcal{M}}_{\text {bifib }}$ such that $[\underline{\mathcal{B}}] \subset\left[\underline{\mathcal{M}}_{\text {bifib }}\right]$ is a strictly full subcategory. Let $\underline{\mathcal{A}}$ be any $\mathcal{V}$-small $\mathcal{K}$-category and let

$$
\delta: \operatorname{DGCAT}_{\mathrm{k}}(\underline{\mathcal{A}}, \underline{\mathcal{B}}) \rightarrow \mathrm{L}_{\mathrm{ohe}} \operatorname{DGCAT}_{\mathrm{k}}(\underline{\mathcal{A}}, \underline{\mathcal{B}})
$$

be the additive $\mathrm{k}$-localization of $\operatorname{DGCAT}_{\mathrm{k}}(\underline{\mathcal{A}}, \underline{\mathcal{B}})$ with respect to the set of objectwise homotopy equivalences (see Corollary 5.6). Let $(\mathbf{i}, \mathbf{l})$ and $\left(\mathbf{i}^{\prime}, \mathbf{\imath}^{\prime}\right)$ be $\mathcal{K}$-enriched $\mathbb{I}$-fibrant resolution functors on $\underline{\mathcal{N}}$. Let $F: \underline{\mathcal{A}} \rightarrow \underline{\mathcal{N}}$ be a $\mathcal{K}$-functor such that $\mathbf{i} F: \underline{\mathcal{A}} \rightarrow \underline{\mathcal{N}}_{\mathbb{I} \text {-fib }}=$ $\underline{\mathcal{M}}_{\text {bifib }}$ lands in $\underline{\mathcal{B}}$. Then $\mathbf{i}^{\prime} F$ and $\mathbf{i}^{\prime} \mathbf{i} F$ and $\mathbf{i i}^{\prime} F$ also land in $\underline{\mathcal{B}}$ and the zig-zags

$$
\begin{aligned}
& \mathbf{i} F \stackrel{\mathbf{\iota}^{\prime} \mathbf{i} F}{\longrightarrow} \mathbf{i}^{\prime} \mathbf{i} F \stackrel{\mathbf{i}^{\prime} \iota F}{\longleftarrow} \mathbf{i}^{\prime} F, \\
& \mathbf{i} F \stackrel{\mathbf{i}^{\prime} F}{\longrightarrow} \mathbf{i i}^{\prime} F \stackrel{\mathbf{i}^{\prime} F}{\longleftarrow} \mathbf{i}^{\prime} F
\end{aligned}
$$

of objectwise homotopy equivalences in $\operatorname{DGCAT}_{\mathrm{k}}(\underline{\mathcal{A}}, \underline{\mathcal{B}})$ give rise to the same isomorphism

$$
\varphi_{\mathbf{i} F \rightarrow \mathbf{i}^{\prime} F}: \mathbf{i} F \stackrel{\sim}{\rightarrow} \mathbf{i}^{\prime} F
$$

in $\mathrm{L}_{\text {ohe }} \operatorname{DGCAT}_{\mathrm{k}}(\underline{\mathcal{A}}, \underline{\mathcal{B}})$ (cf. Remark 5.9).

If $\left(\mathbf{i}^{\prime \prime}, \mathfrak{\iota}^{\prime \prime}\right)$ is another $\mathcal{K}$-enriched $\mathbb{I}$-fibrant resolution functor, we have

$$
\begin{aligned}
\varphi_{\mathbf{i}^{\prime} F \rightarrow \mathbf{i}^{\prime \prime} F} \circ \varphi_{\mathbf{i} F \rightarrow \mathbf{i}^{\prime} F} & =\varphi_{\mathbf{i} F \rightarrow \mathbf{i}^{\prime \prime} F}, \\
\varphi_{\mathbf{i} F \rightarrow \mathbf{i} F} & =\mathrm{id}_{\mathbf{i} F}
\end{aligned}
$$

where the isomorphisms $\varphi_{\mathbf{i}^{\prime} F \rightarrow \mathbf{i}^{\prime \prime} F}, \varphi_{\mathbf{i} F \rightarrow \mathbf{i}^{\prime \prime} F}$ and $\varphi_{\mathbf{i} F \rightarrow \mathbf{i} F}$ are defined in the analog way.

In particular, we obtain

$$
\begin{aligned}
\delta(\mathbf{i} \iota F) & =\delta(\mathbf{\imath} F), \\
\delta\left(\mathbf{i}^{\prime} \iota F\right)^{-1} \delta\left(\iota^{\prime} \mathbf{i} F\right) & =\delta\left(\mathbf{\iota}^{\prime} F\right)^{-1} \delta\left(\mathbf{i}^{\prime} F\right) .
\end{aligned}
$$


Proof. Let $A \in \underline{\mathcal{A}}$ and consider the commutative cube

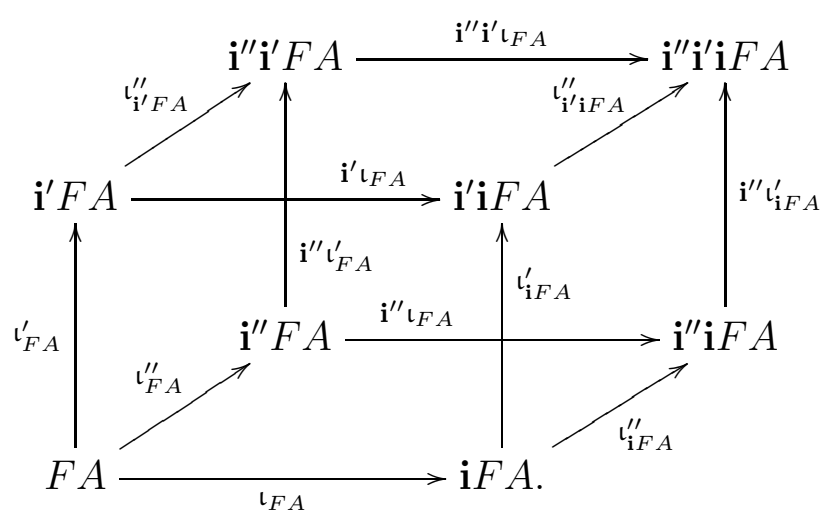

in $\mathcal{M}$. All morphisms are weak equivalences (by the 2 -out-of-3 property), and if we delete the object $F A$ and its outgoing arrows, all remaining morphisms are homotopy equivalences (cf. Remark 6.1 below); since $\mathbf{i} F A$ is in $\mathcal{B}$, all the remaining objects are in $\mathcal{B}$ by our assumption that $[\underline{\mathcal{B}}] \subset\left[\underline{\mathcal{M}}_{\mathbb{I}-\text { fib }}\right]$ is strictly full.

We define $\varphi_{\mathbf{i} F \rightarrow \mathbf{i}^{\prime} F}:=\delta\left(\mathbf{i}^{\prime} \mathbf{\imath} F\right)^{-1} \delta\left(\mathbf{l}^{\prime} \mathbf{i} F\right): \mathbf{i} F \stackrel{\sim}{\rightarrow} \mathbf{i}^{\prime} F$ using the zig-zag (5.19), and in a similar way, we define $\varphi_{\mathbf{i}^{\prime} F \rightarrow \mathbf{i}^{\prime \prime} F}:=\delta\left(\mathbf{i}^{\prime \prime} \mathfrak{\iota}^{\prime} F\right)^{-1} \delta\left(\mathbf{\iota}^{\prime \prime} \mathbf{i}^{\prime} F\right): \mathbf{i}^{\prime} F \stackrel{\sim}{\rightarrow} \mathbf{i}^{\prime \prime} F$ and $\varphi_{\mathbf{i} F \rightarrow \mathbf{i}^{\prime \prime} F}:=$ $\delta\left(\mathbf{i}^{\prime \prime} \mathfrak{l} F\right)^{-1} \delta\left(\mathbf{l}^{\prime \prime} \mathbf{i} F\right): \mathbf{i} F \stackrel{\sim}{\rightarrow} \mathbf{i}^{\prime \prime} F$. Then (5.22) follows immediately from the above cube (without using its corner $F A$ ).

The equality (5.22) for $\left(\mathbf{i}^{\prime \prime}, \mathfrak{l}^{\prime \prime}\right)=\left(\mathbf{i}^{\prime}, \mathbf{l}^{\prime}\right)=(\mathbf{i}, \mathfrak{l})$ says that $\varphi_{\mathbf{i} F \rightarrow \mathbf{i} F}$ is an idempotent isomorphism of $\mathbf{i} F$. This implies (5.23) and (5.24).

From (5.22) for $\left(\mathbf{i}^{\prime \prime}, \mathfrak{l}^{\prime \prime}\right)=(\mathbf{i}, \mathfrak{l})$ and (5.23) we obtain $\varphi_{\mathbf{i}^{\prime} F \rightarrow \mathbf{i} F} \circ \varphi_{\mathbf{i} F \rightarrow \mathbf{i}^{\prime} F}=\varphi_{\mathbf{i} F \rightarrow \mathbf{i} F}=$ $\mathrm{id}_{\mathbf{i} F}$. Rewritten, this just means that (5.25) holds and shows that $\varphi_{\mathbf{i} F \rightarrow \mathbf{i}^{\prime} F}$ can also be defined using the zig-zag (5.20).

The following result is similar and may be skipped on a first reading.

Proposition 5.11. Let $\underline{\mathcal{N}}$ be as above. Let $\underline{\mathcal{B}}$ be a full additive $\mathcal{K}$-subcategory of $\underline{\mathcal{M}}_{\text {bifib }}$ such that $[\underline{\mathcal{B}}] \subset\left[\underline{\mathcal{M}}_{\text {bifib }}\right]$ is a strictly full subcategory. Let $\underline{\mathcal{A}}$ be any $\mathcal{V}$-small $\mathcal{K}$-category and let

$$
\delta: \operatorname{DGCAT}_{\mathrm{k}}(\underline{\mathcal{A}}, \underline{\mathcal{B}}) \rightarrow \mathrm{L}_{\mathrm{ohe}} \operatorname{DGCAT}_{\mathrm{k}}(\underline{\mathcal{A}}, \underline{\mathcal{B}})
$$

be the additive $\mathrm{k}$-localization of $\operatorname{DGCAT}_{\mathrm{k}}(\underline{\mathcal{A}}, \underline{\mathcal{B}})$ with respect to the set of objectwise homotopy equivalences (see Corollary 5.6). Let $\left(\mathcal{Y}, \mathcal{O}_{\mathcal{Y}}\right)$ be a $\mathcal{U}$-small $\mathrm{k}$-ringed site, let $F: \underline{\mathrm{C}}(\mathcal{Y})_{\mathrm{hflat}} \rightarrow \underline{\mathcal{N}}$ be a $\mathcal{K}$-functor which maps quasi-isomorphisms to homotopy equivalences, and let $G: \underline{\mathcal{A}} \rightarrow \underline{\mathrm{C}}(\mathcal{Y})$ be a $\mathcal{K}$-functor. Let $(\mathbf{e}, \epsilon)$ and $\left(\mathbf{e}^{\prime}, \epsilon^{\prime}\right)$ be $\mathcal{K}$ enriched $\mathbb{E}$-fibrant resolution functors on $\underline{\mathrm{C}}(\mathcal{Y})$. Assume that the composition

$$
F \mathbf{e} G: \underline{\mathcal{A}} \stackrel{G}{\rightarrow} \underline{\mathrm{C}}(\mathcal{Y}) \stackrel{\text { e }}{\rightarrow} \underline{\mathbb{E}}(\mathcal{Y}) \subset \underline{\mathrm{C}}(\mathcal{Y})_{\text {hflat }} \stackrel{F}{\rightarrow} \underline{\mathcal{M}}
$$


lands in $\underline{\mathcal{B}}$. Then $F \mathbf{e}^{\prime} G$ and $F \mathbf{e}^{\prime} \mathbf{e} G$ and $F \mathbf{e e}^{\prime} G$ also land in $\underline{\mathcal{B}}$ and the zig-zags

$$
\begin{aligned}
& F \mathbf{e} G \stackrel{F \epsilon^{\prime} \mathbf{e} G}{\longleftarrow} F \mathbf{e}^{\prime} \mathbf{e} G \stackrel{F \mathbf{e}^{\prime} \epsilon G}{\longrightarrow} F \mathbf{e}^{\prime} G \\
& F \mathbf{e} G \stackrel{F \mathbf{e} \epsilon^{\prime} G}{\longleftarrow} F \mathbf{e e}^{\prime} G \stackrel{F \in \mathbf{e}^{\prime} G}{\longrightarrow} F \mathbf{e}^{\prime} G
\end{aligned}
$$

of objectwise homotopy equivalences in $\operatorname{DGCAT}_{\mathrm{k}}(\underline{\mathcal{A}}, \underline{\mathcal{B}})$ give rise to the same isomorphism

$$
\psi_{F \mathbf{e} G \rightarrow F \mathbf{e}^{\prime} G}: F \mathbf{e} G \stackrel{\sim}{\rightarrow} F \mathbf{e}^{\prime} G
$$

in $\mathrm{L}_{\text {ohe }} \operatorname{DGCAT}_{\mathrm{k}}(\underline{\mathcal{A}}, \underline{\mathcal{B}})$. If $\left(\mathbf{e}^{\prime \prime}, \epsilon^{\prime \prime}\right)$ is another $\mathcal{K}$-enriched $\mathbb{E}$-fibrant resolution functor, we have

$$
\begin{aligned}
\psi_{F \mathbf{e}^{\prime} G \rightarrow F \mathbf{e}^{\prime \prime} G} \circ \psi_{F \mathbf{e} G \rightarrow F \mathbf{e}^{\prime} G} & =\psi_{F \mathbf{e} G \rightarrow F \mathbf{e}^{\prime \prime} G}, \\
\psi_{F \mathbf{e} G \rightarrow F \mathbf{e} G} & =\operatorname{id}_{F \mathbf{e} G}
\end{aligned}
$$

where the isomorphisms $\psi_{F \mathbf{e}^{\prime} G \rightarrow F \mathbf{e}^{\prime \prime} G}, \psi_{F \mathbf{e} G \rightarrow F \mathbf{e}^{\prime \prime} G}$ and $\psi_{F \mathbf{e} G \rightarrow F \mathbf{e} G}$ are defined in the analog way. In particular, we obtain

$$
\begin{aligned}
\delta(F \in \mathbf{e} G) & =\delta(F \mathbf{e} \epsilon G), \\
\delta\left(F \mathbf{e}^{\prime} \epsilon G\right) \delta\left(F \epsilon^{\prime} \mathbf{e} G\right)^{-1} & =\delta\left(F \in \mathbf{e}^{\prime} G\right) \delta\left(F \mathbf{e} \epsilon^{\prime} G\right)^{-1} .
\end{aligned}
$$

Proof. Let $A \in \underline{\mathcal{A}}$, put $A^{\prime}:=G(A)$ and consider the commutative diagram

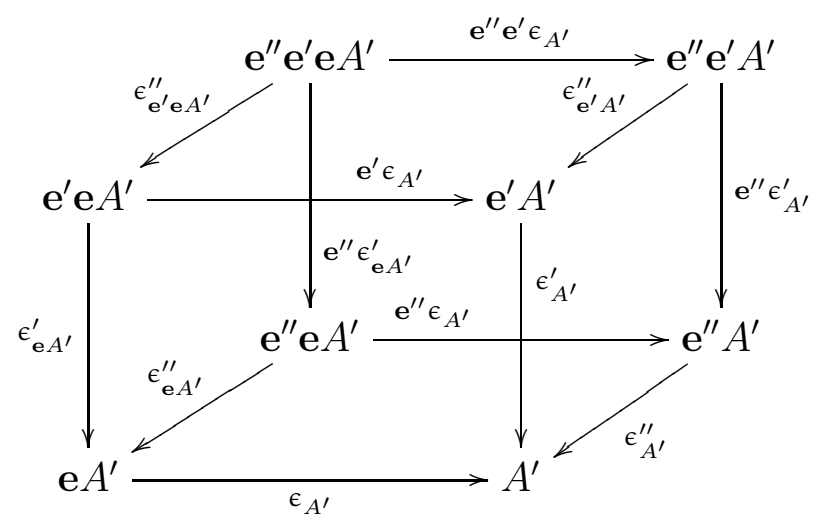

in $\mathrm{C}(\mathcal{Y})$. All morphisms are quasi-isomorphisms, and all objects apart from $A^{\prime}$ are hflat. So if we remove the object $A^{\prime}$ and all morphisms with target $A^{\prime}$ from this diagram and apply $F$ we obtain a commutative diagram of homotopy equivalences in $\mathcal{M}$. Since $[\underline{\mathcal{B}}]$ is a strictly full subcategory of $\left[\underline{\mathcal{M}}_{\mathbb{I} \text {-fib }}\right]$ all objects of this new diagram lie in $\underline{\mathcal{B}}$, and we can define the isomorphism $\psi_{F \mathbf{e} G \rightarrow F \mathbf{e}^{\prime} G}:=\delta\left(F \mathbf{e}^{\prime} \in G\right) \delta\left(F \epsilon^{\prime} \mathbf{e} G\right)^{-1}: F \mathbf{e} G \rightarrow$ $F \mathbf{e}^{\prime} G$. All claims of the proposition are now proved as the corresponding claims in Proposition 5.10. 
5.4. The multicategory of formulas for the four operations. Let $\mathrm{R}$ be a ring and $\mathcal{R}=\mathrm{C}(\mathrm{R})$. Let $\mathrm{fml}_{\mathrm{R}}^{\prime}$ be the free multicategory whose objects are symbols $\mathcal{X}$ and $\mathcal{X}^{\text {op }}$, for each $\mathcal{U}$-small R-ringed site $\left(\mathcal{X}, \mathcal{O}_{\mathcal{X}}\right)$, and whose generating morphisms are given as follows (where all appearing R-ringed sites are assumed to be $\mathcal{U}$-small):

(a) for each $\mathrm{R}$-ringed site $\left(\mathcal{X}, \mathcal{O}_{\mathcal{X}}\right)$ there are morphisms $\otimes:(\mathcal{X}, \mathcal{X}) \rightarrow \mathcal{X}$ and $\mathcal{H o m}:\left(\mathcal{X}^{\text {op }}, \mathcal{X}\right) \rightarrow \mathcal{X}$ and $\mathrm{Hom}:\left(\mathcal{X}^{\text {op }}, \mathcal{X}\right) \rightarrow$ pt where pt is the symbol for the R-ringed site (pt, R);

(b) for each morphism $\alpha:\left(\operatorname{Sh}(\mathcal{Y}), \mathcal{O}_{\mathcal{Y}}\right) \rightarrow\left(\operatorname{Sh}(\mathcal{X}), \mathcal{O}_{\mathcal{X}}\right)$ of R-ringed topoi there are morphisms $\alpha^{*}: \mathcal{X} \rightarrow \mathcal{Y}$ and $\alpha_{*}: \mathcal{Y} \rightarrow \mathcal{X}$

(c) for each R-ringed site $\left(\mathcal{X}, \mathcal{O}_{\mathcal{X}}\right)$ and each object $G \in \mathrm{C}(\mathcal{X})$ there is a morphism $G: \emptyset \rightarrow \mathcal{X}$

(d) each of these morphisms has an "opposite" morphism: there are morphisms $\otimes^{\text {op }}:\left(\mathcal{X}^{\text {op }}, \mathcal{X}^{\text {op }}\right) \rightarrow \mathcal{X}^{\text {op }}, \mathcal{H o m}^{\text {op }}:\left(\mathcal{X}, \mathcal{X}^{\text {op }}\right) \rightarrow \mathcal{X}^{\text {op }}$, Hom $^{\text {op }}:\left(\mathcal{X}, \mathcal{X}^{\text {op }}\right) \rightarrow$ $\mathrm{pt}^{\mathrm{op}},\left(\alpha^{*}\right)^{\mathrm{op}}: \mathcal{X}^{\mathrm{op}} \rightarrow \mathcal{Y}^{\mathrm{op}}, \alpha_{*}^{\mathrm{op}}: \mathcal{Y}^{\mathrm{op}} \rightarrow \mathcal{X}^{\mathrm{op}}$, and $G^{\mathrm{op}}: \emptyset \rightarrow \mathcal{X}^{\mathrm{op}}$.

There is an obvious involution

$$
(-)^{\mathrm{op}}: \mathrm{fml}_{\mathrm{R}}^{\prime} \rightarrow \mathrm{fml}_{\mathrm{R}}^{\prime}
$$

swapping $\mathcal{X}$ and $\mathcal{X}^{\text {op }}$ and exchanging each morphism with its opposite.

If $\sigma$ is the canonical morphism (4.10) we abbreviate $\Gamma_{. .}:=\sigma_{* *}$. The morphisms in $\mathrm{fml}_{\mathrm{R}}^{\prime}$ may be viewed as planar rooted trees. We prefer a formulaic notation, for example $\mathcal{H o m}\left(\alpha^{*}(-),-\right)$ denotes the obvious morphism $\left(\mathcal{X}^{\circ}, \mathcal{Y}\right) \rightarrow \mathcal{Y}$. More precisely, this morphism would be denoted $\mathcal{H o m}\left(\left(\alpha^{*}\right)^{\mathrm{op}}(-),-\right)$.

Remark 5.12. In 7.2 we will enlarge the multicategory fml $_{\mathrm{R}}^{\prime}$ by adding generating morphisms $\alpha_{!}, \alpha^{!}, \alpha^{-1}$ for suitable morphisms $\alpha$ of R-ringed spaces, and call this enlarged multicategory $\mathrm{fml}_{\mathrm{R}}$. In 8 we even add some 2 -morphisms and define a klinear 2-multicategory of formulas.

Remark 5.13. We call $\mathrm{fml}_{\mathrm{R}}^{\prime}$ the multicategory of formulas. A priori this seems to be a bad name: neither the objects nor the morphisms of $\mathrm{fml}_{\mathrm{R}}^{\prime}$ look like formulas. However, in the k-linear 2-multicategory of formulas defined later on there are 2-(iso)morphisms

as $\alpha_{*} \mathcal{H o m}\left(\alpha^{*}(-),-\right) \stackrel{\sim}{\rightarrow} \mathcal{H}$ om $\left(-, \alpha_{*}(-)\right)$ which really look like formulas and hence justify the name.

5.4.1. Interpretation of formulas in triangulated categories. By definition of $\mathrm{fml}_{\mathrm{R}}^{\prime}$, there is a unique interpretation functor

$$
\mathrm{D}: \mathrm{fml}_{\mathrm{R}}^{\prime} \rightarrow \text { trcat }_{\mathrm{R}}
$$

of multicategories given on objects by $\mathcal{X} \mapsto \mathrm{D}(\mathcal{X})$ and $\mathcal{X}^{\text {op }} \mapsto \mathrm{D}(\mathcal{X})^{\mathrm{op}}$, and on generating morphisms by $\otimes \mapsto \otimes^{\mathbf{L}}, \mathcal{H o m} \mapsto \mathbf{R H o m}$, Hom $\mapsto$ RHom, $\alpha^{*} \mapsto \mathbf{L} \alpha^{*}$, $\alpha_{*} \mapsto \mathbf{R} \alpha_{*}$, and $G \mapsto G$ (since $G$ is also an object of $\mathrm{D}(\mathcal{X})$ we can view it as a morphism $G: \emptyset \rightarrow \mathrm{D}(\mathcal{X})$ ), and by mapping the opposites of these morphisms to the 
opposites of their images. This is well-defined since $\mathrm{D}(\mathcal{X})$ is $\mathcal{V}$-small (use for example Proposition 5.5). The notation $\mathrm{D}$ for this functor is justified by $\mathrm{D}(\mathcal{X})=\mathrm{D}(\mathcal{X})$; note that for example $\mathrm{D}\left(\alpha_{*}\right)=\mathbf{R} \alpha_{*}$.

Remark 5.14. In the following, when defining a functor from $\mathrm{fml}_{\mathrm{R}}^{\prime}$ to another multicategory with an obvious involution $(-)^{\text {op }}$, we usually only give the value of this functor on the objects $\mathcal{X}$ and the generating morphisms $\underset{.}{\otimes}, \mathcal{H o m}, \mathrm{Hom}, \alpha^{*}, \alpha_{*}, G$ and extend it tacitly so that it is compatible with the involutions.

\section{Four operations}

We lift the four functor formalism to the dg level, i. e. the part of GrothendieckVerdier-Spaltenstein's six functor formalism concerning the four functors $\otimes^{\mathbf{L}}, \mathbf{R} \mathcal{H}$ om, $\mathbf{L} \alpha^{*}, \mathbf{R} \alpha_{*}$.

We fix a field $\mathrm{k}$ and write $\mathcal{K}=\mathrm{C}(\mathrm{k})$. We use the index "hflat" (resp. "hinj", "whinj") to indicate subcategories of h-flat (resp. h-injective, weakly h-injective) objects.

All ringed sites $(\mathcal{X}, \mathcal{O})$ in this section are assumed to be $\mathcal{U}$-small. In particular, this applies to the ringed sites giving rise to ringed topoi.

6.1. Fixed data. For the rest of this article we fix for each k-ringed site $(\mathcal{X}, \mathcal{O})$ a $\mathcal{K}$-enriched $\mathbb{E}$-cofibrant resolution functor $(\mathbf{e}, \epsilon)=\left(\mathbf{e}_{(\mathcal{X}, \mathcal{O})}, \epsilon_{(\mathcal{X}, \mathcal{O})}\right)$ and a $\mathcal{K}$-enriched $\mathbb{I}$-fibrant resolution functor $(\mathbf{i}, \mathfrak{l})=\left(\mathbf{i}_{(\mathcal{X}, \mathcal{O})}, \mathfrak{l}_{(\mathcal{X}, \mathcal{O})}\right)$, i. e. we have $\mathcal{K}$-functors

$$
\text { e: } \underline{\mathrm{C}}(\mathcal{X}) \rightarrow \underline{\mathbb{E}}(\mathcal{X}) \subset \underline{\mathrm{C}}(\mathcal{X})_{\text {hflat }} \quad \text { and } \quad \mathbf{i}: \underline{\mathrm{C}}(\mathcal{X}) \rightarrow \underline{\mathbb{I}}(\mathcal{X}) \subset \underline{\mathrm{C}}(\mathcal{X})_{\text {hinj }}
$$

and $\mathcal{K}$-natural transformations $\mathbf{e} \stackrel{\epsilon}{\rightarrow}$ id $\stackrel{\llcorner}{\rightarrow}$ i such that for each $M \in \underline{\mathrm{C}}(\mathcal{X})$ the morphism $\epsilon_{M}: \mathbf{e}(M) \rightarrow M$ is a trivial $\mathbb{E}$-fibration and the morphism $\mathfrak{\iota}_{M}: M \rightarrow \mathbf{i}(M)$ is a trivial $\mathbb{I}$-cofibration. This is possible by Theorem 4.17; the above inclusions were observed in Lemmas 4.11 and 4.1.1. For simplicity we assume that the $\mathcal{K}$-enriched resolution functors for $(\mathrm{pt}, \mathrm{k})$ are trivial, i. e. $\left(\mathbf{e}_{(\mathrm{pt}, \mathrm{k})}, \epsilon_{(\mathrm{pt}, \mathrm{k})}\right)=(\mathrm{id}, \mathrm{id})=\left(\mathbf{i}_{(\mathrm{pt}, \mathrm{k})}, \mathrm{l}_{(\mathrm{pt}, \mathrm{k})}\right)$. This is allowed by Lemma 4.25.

Remark 6.1. Here are some trivial facts that are frequently used in the following. Let $(\mathcal{X}, \mathcal{O})$ be a k-ringed site. Any quasi-isomorphism in $\mathrm{C}(\mathcal{X})$ between h-injective objects is invertible in the homotopy category $[\underline{\mathrm{C}}(\mathcal{X})]$. In particular, the $\mathcal{K}$-functor i maps quasi-isomorphisms to homotopy equivalences. The $\mathcal{K}$-functor e obviously maps quasi-isomorphisms to quasi-isomorphisms.

Remark 6.2. Subsequent constructions will depend on the fixed data. Nevertheless the constructions obtained from different choices are easy to compare. We will explain this in two examples, see Remarks 6.11 and 6.12 . 
6.2. Dg k-enhancements considered. If $(\mathcal{X}, \mathcal{O})$ is a k-ringed site then $\mathbb{I}(\mathcal{X})$ is an object of $\mathrm{ENH}_{\mathrm{k}}$. Recall from 4.1.2 that $\mathbb{I}(\mathcal{X})$ together with the equivalence

$$
[\underline{\mathbb{I}}(\mathcal{X})] \stackrel{\sim}{\rightarrow} \mathrm{D}(\mathcal{X})
$$

is a $\mathcal{K}$-enhancement of $\mathrm{D}(\mathcal{X})$. Moreover, this equivalence has

$$
\overline{[\mathbf{i}]}: \mathrm{D}(\mathcal{X}) \stackrel{\sim}{\rightarrow}[\underline{\mathbb{I}}(\mathcal{X})]
$$

as a quasi-inverse, by Lemma 4.7. If $q_{\mathcal{X}}:[\underline{\mathrm{C}}(\mathcal{X})] \rightarrow \mathrm{D}(\mathcal{X})$ denotes the Verdier localization, then $\overline{[\mathbf{i}]}$ is the unique triangulated k-functor such that $\overline{[\mathbf{i}]} q_{\mathcal{X}}=[\mathbf{i}]$.

6.3. Lifts of derived functors. The main players of this section are the following $\mathcal{K}$-functors. If $\alpha:\left(\operatorname{Sh}(\mathcal{Y}), \mathcal{O}_{\mathcal{Y}}\right) \rightarrow\left(\operatorname{Sh}(\mathcal{X}), \mathcal{O}_{\mathcal{X}}\right)$ is a morphism of k-ringed topoi, define $\mathcal{K}$-functors

$$
\begin{aligned}
& \underline{\alpha}^{*}:=\mathbf{i} \alpha^{*} \mathbf{e}: \underline{\mathbb{I}}(\mathcal{X}) \stackrel{\text { e }}{\rightarrow} \underline{\mathbb{E}}(\mathcal{X}) \stackrel{\alpha^{*}}{\rightarrow} \underline{\mathrm{C}}(\mathcal{Y})_{\text {hflat }} \stackrel{\mathrm{i}}{\rightarrow} \underline{\mathbb{I}}(\mathcal{Y}), \\
& \quad \underline{\alpha}_{*}:=\mathbf{i} \alpha_{*}: \underline{\mathbb{I}}(\mathcal{Y}) \stackrel{\alpha_{*}}{\rightarrow} \underline{\mathrm{C}}(\mathcal{X})_{\text {whinj }} \stackrel{\mathrm{i}}{\rightarrow} \underline{\mathbb{I}}(\mathcal{X}) .
\end{aligned}
$$

Here $\alpha^{*}$ and $\alpha_{*}$ are the restrictions of the obvious $\mathcal{K}$-functors $\alpha^{*}: \underline{\mathrm{C}}(\mathcal{X}) \rightarrow \underline{\mathrm{C}}(\mathcal{Y})$ and $\alpha_{*}: \underline{\mathrm{C}}(\mathcal{Y}) \rightarrow \underline{\mathrm{C}}(\mathcal{X})$; they land in the indicated categories by Propositions $\mathrm{A} .2$ and A.9. Note that $\underline{\alpha}^{*}$ and $\underline{\alpha}_{*}$ are 1-morphisms in the k-linear 2-multicategories $\widetilde{\mathrm{ENH}}_{\mathrm{k}}$ and $\mathrm{ENH}_{\mathrm{k}}$.

If $\left(\mathcal{X}, \mathcal{O}_{\mathcal{X}}\right)$ is a k-ringed site, define $\mathcal{K}$-functors

$$
\begin{aligned}
& \underline{\otimes}:=\mathbf{i}(\mathbf{e}(-) \otimes \mathbf{e}(-)): \underline{\mathbb{I}}(\mathcal{X}) \otimes \underline{\mathbb{I}}(\mathcal{X}) \stackrel{\mathbf{e} \otimes \mathbf{e}}{\rightarrow} \underline{\mathbb{E}}(\mathcal{X}) \otimes \underline{\mathbb{E}}(\mathcal{X}) \stackrel{\otimes}{\rightarrow} \underline{\mathrm{C}}(\mathcal{X})_{\text {hflat }} \stackrel{\text { i }}{\rightarrow} \underline{\mathbb{I}}(\mathcal{X}), \\
& \underline{\mathcal{H} o m}:=\mathbf{i} \mathcal{H} \operatorname{lom}(-,-): \underline{\mathbb{I}}(\mathcal{X})^{\text {op }} \otimes \underline{\mathbb{I}}(\mathcal{X}) \stackrel{\mathcal{H o m}(-,-)}{\longrightarrow} \underline{\mathrm{C}}(\mathcal{X})_{\text {whinj }} \stackrel{\mathrm{i}}{\rightarrow} \underline{\mathbb{I}}(\mathcal{X}) .
\end{aligned}
$$

Here we use the fact that the tensor product of two h-flat objects is h-flat and Proposition A.6. Both $\mathcal{K}$-functors $\underline{\otimes}$ and $\underline{\mathcal{H} o m}$ can and will be viewed as 1-morphisms $\underline{Q}:(\underline{\mathbb{I}}(\mathcal{X}), \underline{\mathbb{I}}(\mathcal{X})) \rightarrow \underline{\mathbb{I}}(\mathcal{X})$ and $\underline{\mathcal{H} o m}:\left(\underline{\mathbb{I}}(\mathcal{X})^{\mathrm{op}}, \underline{\mathbb{I}}(\mathcal{X})\right) \rightarrow \underline{\mathbb{I}}(\mathcal{X})$ in $\widetilde{\mathrm{ENH}}_{\mathrm{k}}$ and $\mathrm{ENH}_{\mathrm{k}}$.

Remark 6.3. Note that $\alpha^{*}, \alpha_{*}, \otimes, \mathcal{H}$ om are $\mathcal{K}$-functors. They are not the underlying functors of the $\mathcal{K}$-functors $\underline{\alpha}^{*}, \underline{\alpha}_{*}, \underline{\otimes}, \underline{\mathcal{H} o m}$.

Let $\sigma=\sigma_{\mathcal{X}}:\left(\operatorname{Sh}(\mathcal{X}), \mathcal{O}_{\mathcal{X}}\right) \rightarrow(\operatorname{Sh}(\mathrm{pt}), \mathrm{k})$ be the canonical morphism (4.10) of kringed topoi, so $\Gamma=\sigma_{*}$, by 4.2.3. We define $\underline{\Gamma}:=\underline{\sigma}_{*}$. Our assumption $\mathbf{i}_{(\mathrm{pt}, \mathrm{k})}=\mathrm{id}$ implies that $\underline{\Gamma}=\underline{\sigma}_{*}=\sigma_{*}=\Gamma: \underline{\mathbb{I}}(\mathcal{X}) \rightarrow \underline{\mathcal{K}}=\underline{\mathrm{C}}(\mathrm{pt}, \mathrm{k})=\underline{\mathbb{I}}(\mathrm{pt}, \mathrm{k})$. Define the $\mathcal{K}$-functor

$$
\underline{\operatorname{Hom}}=\underline{\operatorname{Hom}}_{\mathcal{X}}:=\Gamma \mathcal{H} \text { om }: \underline{\mathbb{I}}(\mathcal{X})^{\text {op }} \otimes \underline{\mathbb{I}}(\mathcal{X}) \rightarrow \underline{\mathcal{K}} .
$$

We write $\underline{\operatorname{End}}(I):=\underline{\operatorname{Hom}}(I, I)$.

Remark 6.4. For $I, J \in \underline{I}(\mathcal{X})$ we have $\underline{\operatorname{Hom}}(I, J)=\underline{\mathrm{C}}_{\mathcal{X}}(I, J)$. The image of $\underline{\text { Hom }}$ under $(5.13)$ is the triangulated k-functor $[\underline{\mathrm{Hom}}]:[\underline{\mathbb{I}}(\mathcal{X})]^{\mathrm{op}} \times[\underline{\mathbb{I}}(\mathcal{X})] \rightarrow[\underline{\mathcal{K}}]$; on objects, it coincides with $(6.8)$, i. e. $[\underline{\operatorname{Hom}}](I, J)=\underline{\operatorname{Hom}}(I, J)$ is a complex of k-vector spaces. On the other hand, $\left[\underline{\mathrm{C}}_{\mathcal{X}}\right](I, J)$ is the k-vector space of closed degree zero morphisms $I \rightarrow J$ up to homotopy, i. e. $\left[\underline{\mathrm{C}}_{\mathcal{X}}\right](I, J)=H^{0}([\underline{\operatorname{Hom}}](I, J))=H^{0}(\underline{\operatorname{Hom}}(I, J))$. To 
avoid possible conflicts of notation we will rarely use the symbol $\underline{\mathrm{C}}_{\mathcal{X}}$ for the $\mathcal{K}$-functor $\underline{\mathrm{C}}(\mathcal{X})^{\mathrm{op}} \otimes \underline{\mathrm{C}}(\mathcal{X}) \rightarrow \mathcal{K},(E, F) \mapsto \underline{\mathrm{C}}_{\mathcal{X}}(E, F)$.

All the derived functors $\mathbf{L} \alpha^{*}, \mathbf{R} \alpha_{*}, \otimes^{\mathbf{L}}, \mathbf{R} \mathcal{H}$ om, $\mathbf{R H o m}$ exist and can be computed in the expected way, by Propositions A.13 and A.14. It is intuitively clear that the

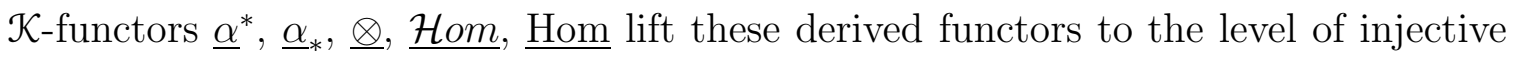
enhancements. Let us confirm this intuition.

Proposition 6.5. If $\alpha:\left(\operatorname{Sh}(\mathcal{Y}), \mathcal{O}_{\mathcal{Y}}\right) \rightarrow\left(\operatorname{Sh}(\mathcal{X}), \mathcal{O}_{\mathcal{X}}\right)$ is a morphism of k-ringed topoi, there are canonical 2-isomorphisms

$$
\begin{aligned}
& \omega_{\alpha^{*}}:\left[\underline{\alpha}^{*}\right] \overline{[\mathbf{i}]} \stackrel{\sim}{\rightarrow} \overline{[\mathbf{i}]} \mathbf{L} \alpha^{*}, \\
& \omega_{\alpha_{*}}:\left[\underline{\alpha}_{*}\right] \overline{\mathbf{i}]} \stackrel{\sim}{\rightarrow} \overline{[\mathbf{i}]} \mathbf{R} \alpha_{*}
\end{aligned}
$$

in TRCAT $_{\mathrm{k}}$ as illustrated in the diagrams
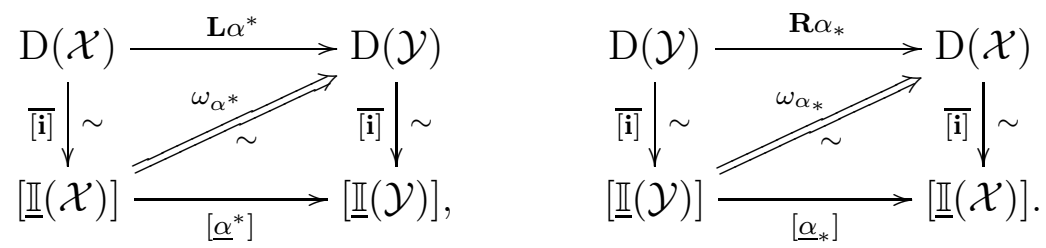

In particular, for $\alpha=\sigma$ there is a canonical 2-isomorphism $\left.\omega_{\Gamma}:[\underline{\Gamma}] \overline{\mathbf{i}}\right] \stackrel{\sim}{\rightarrow} \mathbf{R} \Gamma$.

If $(\mathcal{X}, \mathcal{O})$ is a k-ringed site, there are canonical 2-isomorphisms $\omega_{\otimes}, \omega_{\mathcal{H} o m}$, and $\omega_{\text {Hom }}$ in TRCAT $_{\mathrm{k}}$ as illustrated in the diagrams
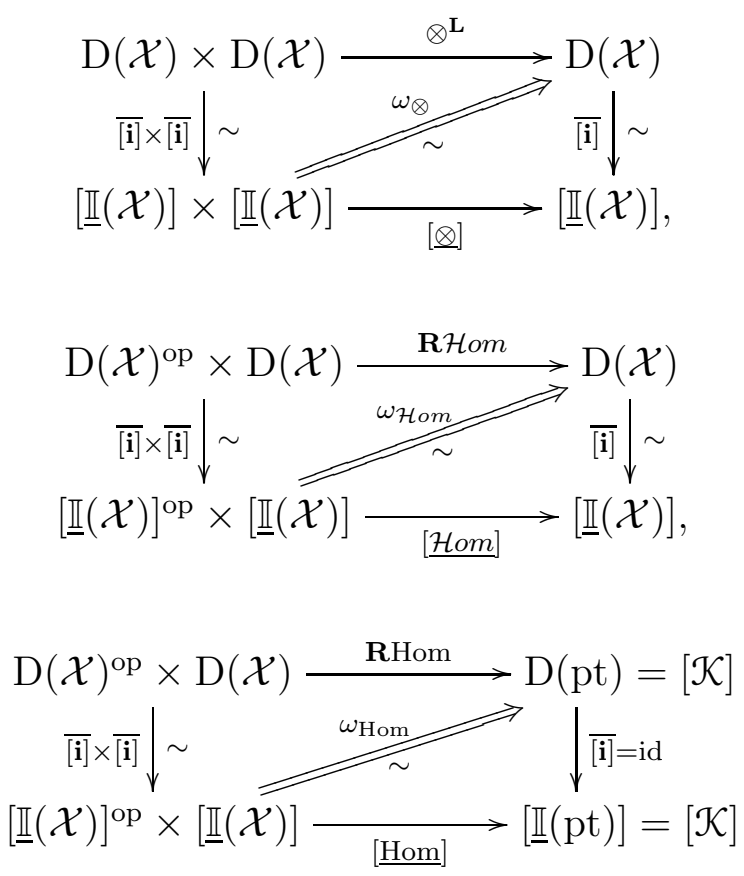

(cf. (5.5) for the definition of the lower horizontal arrows). 
Let us caution the reader that the vertical arrows $\overline{[\mathbf{i}]}$ in the above diagrams are equivalences and not 1-isomorphisms in general even though they are labeled $\sim$.

The canonical 2-isomorphisms $\omega_{\alpha_{*}}$ and $\omega_{\alpha^{*}}$ can even be uniquely characterized, see Propositions 6.8 and 6.9 below. There are similar characterizations of $\omega_{\otimes}, \omega_{\mathcal{H} \text { om }}, \omega_{\text {Hom }}$ left to the reader.

Proof. Construction of $\omega_{\alpha_{*}}$. We can assume without loss of generality that $\mathbf{R} \alpha_{*}=$ $q_{\mathcal{X}}\left[\alpha_{*}\right][\overline{\mathbf{i}}]$. Then $\overline{[\mathbf{i}]} \mathbf{R} \alpha_{*}=\overline{[\mathbf{i}]} q_{\mathcal{X}}\left[\alpha_{*}\right][\overline{\mathbf{i}}]=[\mathbf{i}]\left[\alpha_{*}\right] \overline{[\mathbf{i}]}=\left[\underline{\alpha}_{*}\right] \overline{[\mathbf{i}]}$ and we define $\omega_{\alpha_{*}}$ to be the identity.

Construction of $\omega_{\alpha^{*}}$. Consider the obvious commutative diagram

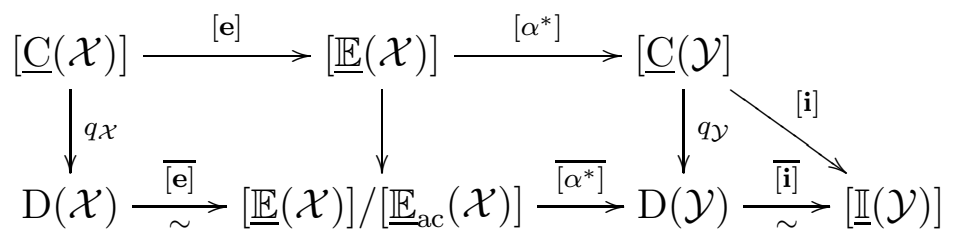

whose lower horizontal arrows are the induced triangulated k-functors and $\overline{[\mathbf{e}]}$ is an equivalence. Without loss of generality we can assume that $\mathbf{L} \alpha^{*}=\overline{\left[\alpha^{*}\right]} \overline{[\mathbf{e}]}$. Then $\overline{[\mathbf{i}]} \mathbf{L} \alpha^{*} q_{\mathcal{X}}=[\mathbf{i}]\left[\alpha^{*}\right][\mathbf{e}]$ by the above diagram. For each object $E \in \mathrm{C}(\mathcal{X})$ the morphism

$$
\mathbf{i} \alpha^{*} \mathbf{e} E \stackrel{\mathbf{i} \alpha^{*} \iota_{E}}{\longrightarrow} \mathbf{i} \alpha^{*} \mathbf{e i} E=\underline{\alpha}^{*} \mathbf{i} E
$$

in $\mathbb{I}(\mathcal{Y})$ is a homotopy equivalence by Remark 6.1 and Proposition A.2, i. e. an isomorphism in $[\mathbb{I}(\mathcal{Y})]$. The family of these isomorphisms defines a natural isotransformation $\left.\overline{[\mathbf{i}]} \mathbf{L} \alpha^{*} \stackrel{\sim}{\rightarrow}\left[\underline{\alpha}^{*}\right] \overline{\mathbf{i}]}\right]$ of triangulated k-functors $\mathrm{D}(\mathcal{X}) \rightarrow[\underline{\mathbb{I}}(\mathcal{Y})]$. We let $\omega_{\alpha^{*}}$ be the inverse of this isotransformation.

Construction of $\omega_{\mathcal{H} \text { om }}$ and $\omega_{\text {Hom. }}$. We can assume that

$$
\mathbf{R} \mathcal{H o m}(-,-)=q_{\mathcal{X}}[\mathcal{H o m}](-, \overline{\mathbf{i}]}(-)) .
$$

For $E, F \in \mathrm{C}(\mathcal{X})$ the morphism

$$
\underline{\mathcal{H} o m}(\mathbf{i} E, \mathbf{i} F)=\mathbf{i} \mathcal{H} \operatorname{om}(\mathbf{i} E, \mathbf{i} F) \stackrel{\mathbf{i} \mathcal{H} o m\left(\iota_{E}, \operatorname{id}_{\mathbf{i}} F\right)}{\longrightarrow} \mathbf{i} \mathcal{H} \operatorname{om}(E, \mathbf{i} F)
$$

defines an isomorphism in $[\mathbb{I}(\mathcal{X})]$, by Proposition A.5.(a). We define $\omega_{\mathcal{H} \text { om }}$ to be the family of these isomorphisms. Similarly, $\omega_{\text {Hom }}$ is constructed using the homotopy equivalences $\underline{\operatorname{Hom}}(\mathbf{i} E, \mathbf{i} F)=\underline{\mathrm{C}}_{\mathcal{X}}(\mathbf{i} E, \mathbf{i} F) \stackrel{\underline{\mathrm{C}}_{\mathcal{X}}\left({ }^{\left({ }_{E}, \mathrm{id}_{\mathbf{i} F)}\right.} \longrightarrow\right.}{\mathrm{C}_{\mathcal{X}}}(E, \mathbf{i} F)$.

Construction of $\omega_{\otimes}$. We can assume that $\left((-) \otimes^{\mathbf{L}}(-)\right)=(\overline{\mathbf{e}}(-) \bar{\otimes} \overline{\mathbf{e}}(-))$ where $\bar{\otimes}:[\underline{\mathbb{E}}(\mathcal{X})] /\left[\underline{\mathbb{E}}_{\mathrm{ac}}(\mathcal{X})\right] \times[\underline{\mathbb{E}}(\mathcal{X})] /\left[\underline{\mathbb{E}}_{\mathrm{ac}}(\mathcal{X})\right] \rightarrow \mathrm{D}(\mathcal{X})$ is the obvious functor, cf. Proposition A.1. This proposition also shows that the morphism

$$
\mathbf{i}(\mathbf{e} E \otimes \mathbf{e} F) \stackrel{\mathbf{i}\left(\mathbf{e \iota}_{E} \otimes \mathbf{e}_{F}\right)}{\longrightarrow} \mathbf{i}(\mathbf{e i} E \otimes \mathbf{e i} F)=\mathbf{i} E \underline{\otimes} \mathbf{i} F
$$

defines an isomorphism in $[\underline{I}(\mathcal{X})]$, for $E, F \in \mathrm{C}(\mathcal{X})$. Let $\omega_{\otimes}$ be given by the inverses of these isomorphisms. 
Remark 6.6. If $\alpha:\left(\operatorname{Sh}(\mathcal{Y}), \mathcal{O}_{\mathcal{Y}}\right) \rightarrow\left(\operatorname{Sh}(\mathcal{X}), \mathcal{O}_{\mathcal{X}}\right)$ is a flat morphism of k-ringed topoi, then $\underline{\alpha}^{*} I=\mathbf{i} \alpha^{*} \mathbf{e} I \stackrel{\mathrm{i} \alpha^{*} \epsilon_{I}}{\longrightarrow} \mathbf{i} \alpha^{*} I$ is a homotopy equivalence for all $I \in \mathbb{I}(\mathcal{X})$. Therefore $\underline{\alpha}^{*} \rightarrow \mathbf{i} \alpha^{*}$ is an objectwise homotopy equivalence and defines a 2-isomorphism $\underline{\alpha}^{*} \stackrel{\sim}{\rightarrow}$ i $\alpha^{*}$ in $\mathrm{ENH}_{\mathrm{k}}$.

Let $\left(\mathcal{X}, \mathcal{O}_{\mathcal{X}}\right)$ be a k-ringed site. If $G \in \mathrm{C}(\mathcal{X})$ is an object we define

$$
\underline{G}:=\mathrm{i} G .
$$

This is an object of $\mathbb{I}(\mathcal{X})$ and of $[\mathbb{I}(\mathcal{X})]$ and can therefore be viewed as a 1-morphism in $\widetilde{\mathrm{ENH}}_{\mathrm{k}}$ and $\mathrm{ENH}_{\mathrm{k}}$, by Remark 5.8. Trivially, the diagram

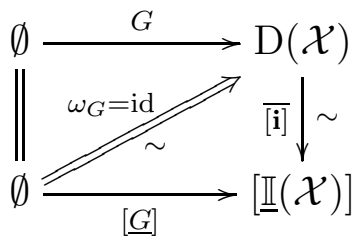

in TRCAT $_{\mathrm{k}}$ commutes where $\omega_{G}:=\mathrm{id}:[\underline{G}] \stackrel{\rightrightarrows}{\rightarrow} \overline{\mathrm{i}]} G$.

Remark 6.7. For $G \in \mathcal{K}=\mathrm{C}(\mathrm{pt}, \mathrm{k})$ we have $\underline{G}=G$ by assumption $\mathbf{i}_{(\mathrm{pt}, \mathrm{k})}=\mathrm{id}$, in particular $\underline{\mathcal{O}}_{\mathrm{pt}}=\mathrm{k}$. If $\left(\mathcal{X}, \mathcal{O}_{\mathcal{X}}\right)$ is a k-ringed site and $\sigma=\sigma_{\mathcal{X}}:\left(\operatorname{Sh}(\mathcal{X}), \mathcal{O}_{\mathcal{X}}\right) \rightarrow$ $(\mathrm{Sh}(\mathrm{pt}), \mathrm{k})$ is the associated morphism of $\mathrm{k}$-ringed topoi (see (4.10)) then

$$
\underline{\sigma}^{*} \underline{\mathcal{O}}_{\mathrm{pt}}=\underline{\sigma}^{*} \mathrm{k}=\mathrm{i} \alpha^{*} \mathrm{ek}=\mathrm{i} \alpha^{*} \mathrm{k}=\mathrm{i} \mathcal{O}_{\mathcal{X}}=\underline{\mathcal{O}}_{\mathcal{X}}=\underline{\mathcal{O}}
$$

using $\mathbf{e}_{(\mathrm{pt}, \mathrm{k})}=\mathrm{id}$. This is a special case of the morphism (6.92) in Lemma 6.31 below.

6.3.1. Some uniqueness results. This subsubsection may be skipped on a first reading. We provide the characterizations of $\omega_{\alpha_{*}}$ and $\omega_{\alpha^{*}}$ mentioned above. All derived functors are taken with respect to the obvious full subcategories of acyclic objects.

Proposition 6.8. Let $\alpha:\left(\operatorname{Sh}(\mathcal{Y}), \mathcal{O}_{\mathcal{Y}}\right) \rightarrow\left(\operatorname{Sh}(\mathcal{X}), \mathcal{O}_{\mathcal{X}}\right)$ be a morphism of k-ringed topoi. Consider the $\mathcal{K}$-natural transformation

$$
\mathbf{i} \alpha_{*} \mathrm{l}: \mathbf{i} \alpha_{*} \rightarrow \mathbf{i} \alpha_{*} \mathbf{i}=\underline{\alpha}_{*} \mathbf{i}: \underline{\mathrm{C}}(\mathcal{Y}) \rightarrow \underline{\mathbb{I}}(\mathcal{X})
$$

and the induced transformation

$$
\left.\left[\mathbf{i} \alpha_{*} l\right]:\left[\mathbf{i} \alpha_{*}\right] \rightarrow\left[\underline{\alpha}_{*} \mathbf{i}\right]=\left[\underline{\alpha}_{*}\right] \overline{\mathbf{i}}\right] q_{\mathcal{Y}}:[\underline{\mathrm{C}}(\mathcal{Y})] \rightarrow[\mathbb{I}(\mathcal{X})]
$$

of triangulated $\mathbf{k}$-functors where we use $[\mathbf{i}]=\overline{[\mathbf{i}}] q_{\mathcal{Y}}$. Then the pair $\left.\left(\left[\underline{\alpha}_{*}\right] \overline{\mathbf{i}}\right],\left[\mathbf{i} \alpha_{*} l\right]\right)$ is a right derived functor of $\left[\mathbf{i} \alpha_{*}\right]:[\underline{\mathrm{C}}(\mathcal{Y})] \rightarrow[\mathbb{I}(\mathcal{X})]$. In particular, if $\left(\mathbf{R} \alpha_{*}, \rho\right)$ is a right derived functor of $q_{\mathcal{X}}\left[\alpha_{*}\right]:[\underline{\mathrm{C}}(\mathcal{Y})] \rightarrow \mathrm{D}(\mathcal{X})$ where $\rho: q_{\mathcal{X}}\left[\alpha_{*}\right] \rightarrow\left(\mathbf{R} \alpha_{*}\right) q_{\mathcal{Y}}$ then there is a unique isotransformation $\omega:\left[\underline{\alpha}_{*}\right] \overline{\mathbf{i}]} \stackrel{\sim}{\rightarrow} \overline{\mathbf{i}]}\left(\mathbf{R} \alpha_{*}\right)$ of triangulated $\mathbf{k}$-functors such that 
the diagram

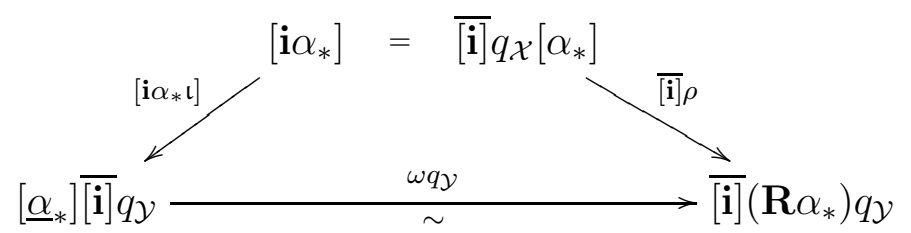

commutes. This isotransformation $\omega$ coincides with the canonical isotransformation (6.10) constructed in the proof of Proposition 6.5.

Proof. The first claim is obvious. Since $\left(\overline{[\mathbf{i}]}\left(\mathbf{R} \alpha_{*}\right), \overline{[\mathbf{i}]} \rho\right)$ is a right derived functor of $\left[\mathbf{i} \alpha_{*}\right]:[\underline{\mathrm{C}}(\mathcal{Y})] \rightarrow[\underline{\mathbb{I}}(\mathcal{X})]$ the second claim follows from the universal property of a right derived functor. To see the last claim, note that $\left.q_{\mathcal{X}}\left[\alpha_{*}\right] \overline{\mathbf{i}]}\right]$ together with $q_{\mathcal{X}}\left[\alpha_{*}\right][\mathbf{l}]: q_{\mathcal{X}}\left[\alpha_{*}\right] \rightarrow q_{\mathcal{X}}\left[\alpha_{*}\right][\overline{\mathbf{i}}] q_{\mathcal{Y}}=q_{\mathcal{X}}\left[\alpha_{*}\right][\mathbf{i}]$ is a right derived functor of $q_{\mathcal{X}}\left[\alpha_{*}\right]$. In this case both isotransformations $\omega$ and $\omega_{\alpha_{*}}$ are obviously the identity.

Proposition 6.9. Let $\alpha:\left(\operatorname{Sh}(\mathcal{Y}), \mathcal{O}_{\mathcal{Y}}\right) \rightarrow\left(\operatorname{Sh}(\mathcal{X}), \mathcal{O}_{\mathcal{X}}\right)$ be a morphism of $\mathrm{k}$-ringed topoi. Consider the zig-zag of $\mathcal{K}$-natural transformations

$$
\underline{\alpha}^{*} \mathbf{i}=\mathbf{i} \alpha^{*} \mathbf{e i} \stackrel{\mathbf{i} \alpha^{*} e \iota}{\longleftarrow} \mathbf{i} \alpha^{*} \mathbf{e} \stackrel{\mathbf{i} \alpha^{*} \epsilon}{\longrightarrow} \mathbf{i} \alpha^{*}: \underline{\mathrm{C}}(\mathcal{X}) \rightarrow \underline{\mathbb{I}}(\mathcal{Y}) .
$$

Then the transformation on the left becomes invertible on homotopy categories, and we obtain the transformation

$$
\left.\left[\mathbf{i} \alpha^{*} \epsilon\right] \circ\left(\left[\mathbf{i} \alpha^{*} \mathbf{e l}\right]\right)^{-1}:\left[\underline{\alpha}^{*} \mathbf{i}\right]=\left[\underline{\alpha}^{*}\right] \overline{\mathbf{i}}\right] q_{\mathcal{X}} \rightarrow\left[\mathbf{i} \alpha^{*}\right]:[\underline{\mathrm{C}}(\mathcal{X})] \rightarrow[\underline{\mathbb{I}}(\mathcal{Y})]
$$

of triangulated $\mathbf{k}$-functors. Then the pair $\left(\left[\underline{\alpha}^{*}\right] \overline{\mathbf{i}]},\left[\mathbf{i} \alpha^{*} \epsilon\right] \circ\left(\left[\mathbf{i} \alpha^{*} \mathbf{e} \mathbf{\imath}\right]\right)^{-1}\right)$ is a left derived functor of $\left[\mathbf{i} \alpha^{*}\right]:[\underline{\mathrm{C}}(\mathcal{X})] \rightarrow[\underline{\mathbb{I}}(\mathcal{Y})]$. In particular, if $\left(\mathbf{L} \alpha^{*}, \lambda\right)$ is a left derived functor of $q_{\mathcal{Y}}\left[\alpha^{*}\right]:[\underline{\mathrm{C}}(\mathcal{X})] \rightarrow \mathrm{D}(\mathcal{Y})$ where $\lambda:\left(\mathbf{L} \alpha^{*}\right) q_{\mathcal{X}} \rightarrow q_{\mathcal{Y}}\left[\alpha^{*}\right]$ then there is a unique isotransformation $\omega:\left[\underline{\alpha}^{*}\right] \overline{\mathbf{i}]} \stackrel{\sim}{\rightarrow} \overline{[\mathbf{i}]}\left(\mathbf{L} \alpha^{*}\right)$ of triangulated $\mathbf{k}$-functors such that the diagram

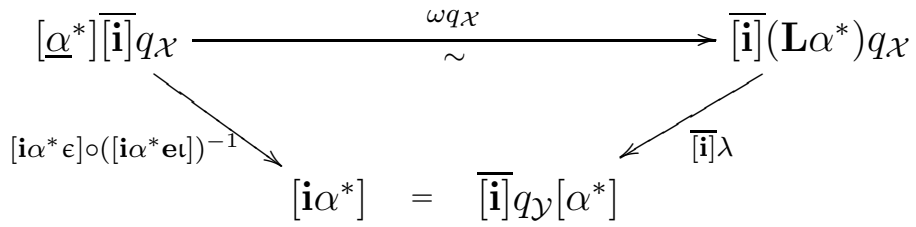

commutes. This isotransformation $\omega$ coincides with the canonical isotransformation (6.9) constructed in the proof of Proposition 6.5.

Proof. We have seen in (6.16) in the proof of Proposition 6.5 that the morphism $\left[\mathbf{i} \alpha^{*} \mathbf{e \iota}_{E}\right]$ in $[\mathbb{I}(\mathcal{Y})]$ is invertible for any $E \in \mathrm{C}(\mathcal{X})$. With notation from the commutative diagram (6.15),

$$
\left(\overline{\left[\alpha^{*}\right]} \overline{[\mathbf{e}]}, \overline{\left[\alpha^{*}\right]} \overline{[\mathbf{e}]} q_{\mathcal{X}}=q_{\mathcal{Y}}\left[\alpha^{*} \mathbf{e}\right] \stackrel{q_{\mathcal{Y}}\left[\alpha^{*} \epsilon\right]}{\longrightarrow} q_{\mathcal{Y}}\left[\alpha^{*}\right]\right)
$$

is a left derived functor of $q_{\mathcal{Y}}\left[\alpha^{*}\right]$, and, by postcomposing with $\overline{[\mathbf{i}]}$,

$$
\left(\overline{[\mathbf{i}]} \overline{\left[\alpha^{*}\right]} \overline{[\mathbf{e}]}, \overline{[\mathbf{i}]} \overline{\left[\alpha^{*}\right]} \overline{[\mathbf{e}]} q_{\mathcal{X}}=\left[\mathbf{i} \alpha^{*} \mathbf{e}\right] \stackrel{\left[\mathbf{i} \alpha^{*} \epsilon\right]}{\longrightarrow}\left[\mathbf{i} \alpha^{*}\right]\right)
$$


is a left derived functor of $\left[\mathbf{i} \alpha^{*}\right]$. This implies that the pair in the proposition is a left derived functor of $\left[\mathbf{i} \alpha^{*}\right]$. The same statement is true for $\left(\overline{[\mathbf{i}]}\left(\mathbf{L} \alpha^{*}\right), \overline{[\mathbf{i}]} \lambda\right)$ and implies the second claim. The last claim is now immediate from the construction of $\omega_{\alpha^{*}}$.

Remark 6.10. If we take (6.29) as the left derived functor of $q_{\mathcal{Y}}\left[\alpha^{*}\right]$ the isotransformation $\omega_{\alpha^{*}}$ is given by

$$
\omega_{\alpha^{*}}=\overline{[\mathbf{i}]} \overline{\left[\alpha^{*}\right]} \overline{[\mathbf{e}]} \overline{[\mathbf{l}]}^{-1}:\left[\underline{\alpha}^{*}\right] \overline{[\mathbf{i}]}=\overline{[\mathbf{i}]} \overline{\left[\alpha^{*}\right]} \overline{[\mathbf{e}]} q_{\mathcal{X}} \overline{[\mathbf{i}]} \stackrel{\sim}{\rightarrow} \overline{[\mathbf{i}]} \overline{\left[\alpha^{*}\right]} \overline{[\mathbf{e}]}
$$

where $\overline{[\mathbf{l}]}:$ id $\rightarrow q_{\mathcal{X}} \overline{[\mathbf{i}]}$ is the obvious isotransformation induced by $\mathbf{\imath}$. This is of course the same isotransformation we constructed in the proof of Proposition 6.5.

6.3.2. Dependence on choices. This subsubsection may be skipped on a first reading.

Remark 6.11. We explain Remark 6.2 for $\underline{\alpha}_{*}$. Let $\left(\mathbf{i}^{\prime}, \mathbf{l}^{\prime}\right)=\left(\mathbf{i}_{(\mathcal{X}, \mathcal{O})}^{\prime}, \mathbf{l}_{(\mathcal{X}, \mathcal{O})}^{\prime}\right)$ be another choice of a $\mathcal{K}$-enriched $\mathbb{I}$-fibrant resolution functor. Then Proposition 5.10 provides a 2-isomorphism $\underline{\alpha}_{*}=\mathbf{i} \alpha_{*} \stackrel{\sim}{\rightarrow} \mathbf{i}^{\prime} \alpha_{*}$ between 1-morphisms $\underline{\mathbb{I}}(\mathcal{Y}) \rightarrow \underline{\mathbb{I}}(\mathcal{X})$ in $\mathrm{ENH}_{\mathrm{k}}$, and the 2 -isomorphisms obtained in this way are compatible in the expected manner, $\mathrm{cf}$. (5.22), (5.23).

Remark 6.12. We explain Remark 6.2 for $\underline{\alpha}^{*}$. Let $\left(\mathbf{e}^{\prime}, \epsilon^{\prime}\right)=\left(\mathbf{e}_{(\mathcal{X}, \mathcal{O})}^{\prime}, \epsilon_{(\mathcal{X}, \mathcal{O})}^{\prime}\right)$ be another $\mathcal{K}$-enriched $\mathbb{E}$-cofibrant resolution functor. Then Proposition 5.11 provides a 2-isomorphism $\psi: \underline{\alpha}^{*}=\mathbf{i} \alpha^{*} \mathbf{e} \stackrel{\sim}{\rightarrow} \mathbf{i} \alpha^{*} \mathbf{e}^{\prime}$ between 1-morphisms $\underline{\mathbb{I}}(\mathcal{X}) \rightarrow \underline{\mathbb{I}}(\mathcal{Y})$ in $\mathrm{ENH}_{\mathrm{k}}$, and the 2 -isomorphisms obtained in this way are compatible as expected, cf. (5.32), (5.33). Similarly, using Proposition 5.10, one may also include different choices of $\mathcal{K}$-enriched $\mathbb{I}$-fibrant resolution functors into this discussion.

6.4. Interpretation of formulas in enhancements. Recall that $\operatorname{enh}_{k}$ denotes the underlying multicategory of the k-linear 2-multicategory $\mathrm{ENH}_{\mathrm{k}}$. Recall the category $\mathrm{fml}_{\mathrm{k}}^{\prime}$ of formulas from 5.4 and the interpretation functor D: fml $_{k}^{\prime} \rightarrow$ trcat $_{\mathrm{k}}$. Similarly, there is a unique interpretation functor

$$
\underline{I}: \mathrm{fml}_{\mathrm{k}}^{\prime} \rightarrow \mathrm{enh}_{\mathrm{k}}
$$

of multicategories given on objects by $\mathcal{X} \mapsto \mathbb{I}(\mathcal{X})$ and on generating morphisms by $\underline{Q} \mapsto \underline{Q}, \underline{\mathcal{H} o m} \mapsto \underline{\mathcal{H} o m}, \alpha^{*} \mapsto \underline{\alpha}^{*}, \alpha_{*} \mapsto \underline{\alpha}_{*}, \underline{\text { Hom }} \mapsto \underline{\text { Hom }}, \underline{G} \mapsto \underline{G}$ (cf. Remark 5.14). The notation $\mathbb{I}$ for this functor is justified by $\mathbb{I}(\mathcal{X})=\underline{\mathbb{I}}(\mathcal{X})$; note that for example $\underline{I}\left(\alpha_{*}\right)=\underline{\alpha}_{*}$. Generalizing this we abbreviate $\underline{t}:=\mathbb{I}(t)$ if $t .$. is any morphism in $\mathrm{fml}_{\mathrm{k}}^{\prime}$.

6.4.1. Relation to interpretation of formulas in triangulated categories. Let $[\mathbb{I}]: \mathrm{fml}_{\mathrm{k}}^{\prime} \rightarrow$ trcat $_{\mathrm{k}}$ denote the composition of (6.32) with (5.15), mapping $\mathcal{X}$ to $[\mathbb{I}(\mathcal{X})]$ and, for example, $\alpha^{*}$ to $\left[\underline{\alpha}^{*}\right]$. Then there is a unique pseudo-natural transformation (see [Bor94, 7.5], generalized to 2-multicategories; here we view the functors D and [I] of multicategories in the trivial way as functors of 2-multicategories)

$$
(\overline{[\mathbf{i}]}, \omega): \mathrm{D} \rightarrow[\underline{\mathbb{I}}]
$$


that maps an object $\mathcal{X}$ (resp. $\mathcal{X}^{\text {op }}$ ) to the 1-morphism (6.3) (resp. its opposite) (which is an equivalence of triangulated k-categories) and that maps the generating morphisms $\alpha^{*}, \alpha_{*}, \otimes, \mathcal{H}$ om, Hom, $G$ to the 2-isomorphisms in the diagrams (6.11), (6.12), (6.13), (6.14), (6.21), respectively, and accordingly for the opposite generating morphisms. If $t$ is an arbitrary morphism in $\mathrm{fml}_{\mathrm{k}}^{\prime}$ the corresponding 2-isomorphism is denoted

$$
\omega_{t}:[\underline{t}] \overline{[\mathbf{i}]} \stackrel{\sim}{\rightarrow} \overline{[\mathbf{i}]} \mathrm{D}(t)
$$

For example, $\omega_{\alpha^{*}}=\omega_{\alpha^{*}}$ and

$$
\omega_{\mathcal{H o m}\left(\alpha^{*}(-),-\right)}: \underline{\mathcal{H} o m}\left(\underline{\alpha}^{*} \overline{\mathbf{i}]}(-), \overline{[\mathbf{i}]}(-)\right) \stackrel{\sim}{\rightarrow} \overline{[\mathbf{i}]} \mathbf{R} \mathcal{H} o m\left(\mathbf{L} \alpha^{*}(-),-\right) .
$$

Example 6.13. The 2-isomorphism $\omega_{\alpha_{*} \alpha^{*}}$ is obtained by juxtaposing the two diagrams in $(6.11)$, i. e. it is the composition

$$
\omega_{\alpha_{*} \alpha^{*}}:\left[\underline{\alpha}_{*}\right]\left[\underline{\alpha}^{*}\right] \overline{\mathbf{i}]} \stackrel{\left[\underline{\alpha}_{*}\right] \omega_{\alpha^{*}}}{\sim}\left[\underline{\alpha}_{*}\right] \overline{\mathbf{i}]} \mathbf{L} \alpha^{*} \stackrel{\omega_{\alpha_{*}} \mathbf{L} \alpha^{*}}{\sim} \overline{[\mathbf{i}]} \mathbf{R} \alpha_{*} \mathbf{L} \alpha^{*} .
$$

Similarly, the 2-isomorphism $\omega_{\alpha^{*} \alpha_{*}}$ is the composition

$$
\omega_{\alpha^{*} \alpha_{*}}:\left[\underline{\alpha}^{*}\right]\left[\underline{\alpha}_{*}\right] \overline{\mathbf{i}]} \stackrel{\left[\underline{\alpha}^{*}\right] \omega_{\alpha_{*}}}{\sim}\left[\underline{\alpha}^{*}\right] \overline{[\mathbf{i}]} \mathbf{R} \alpha_{*} \stackrel{\omega_{\alpha^{*}} \mathbf{R} \alpha_{*}}{\sim} \overline{[\mathbf{i}]} \mathbf{L} \alpha^{*} \mathbf{R} \alpha_{*}
$$

If we choose $\mathbf{R} \alpha_{*}$ as in the proof of Proposition 6.5, then $\omega_{\alpha_{*}}$ is the identity and we have $\omega_{\alpha_{*} \alpha^{*}}=\left[\underline{\alpha}_{*}\right] \omega_{\alpha^{*}}$ and $\omega_{\alpha^{*} \alpha_{*}}=\omega_{\alpha^{*}} \mathbf{R} \alpha_{*}$.

Remark 6.14. Later on, in 8, we will extend fml to a k-linear 2-multicategory $\mathrm{FML}_{\mathrm{k}}$, the functors D and $\mathbb{I}$ (and $[\mathbb{I}]$ ) to functors of k-linear 2-multicategories, and $(\overline{[\mathbf{i}]}, \omega)$ to a pseudo-natural transformation $\mathrm{D} \rightarrow[\mathbb{I}]$ between these extensions, see Theorem 8.5. This theorem summarizes many results of this article.

\subsection{Lifts of relations.}

\subsubsection{Lifts of 2-(iso)morphisms.}

Definition 6.15. Let $v, w:\left(\left(\mathcal{X}_{1}\right)^{\varepsilon_{1}}, \ldots,\left(\mathcal{X}_{n}\right)^{\varepsilon_{n}}\right) \rightarrow \mathcal{Y}^{\delta}$ be morphisms in $\mathrm{fml}_{\mathrm{k}}^{\prime}$. We say that a 2-morphism $\tau: \underline{v}=\underline{\mathbb{I}}(\underline{v}) \rightarrow \underline{w}=\underline{\mathbb{I}}(\underline{w})$ in $\operatorname{ENH}_{\mathrm{k}}(\underline{v}, \underline{w})$-enhances a 2morphism $\sigma: \mathrm{D}\left(\underline{v)} \rightarrow \mathrm{D}(\underline{w})\right.$ in $\mathrm{TRCAT}_{\mathrm{k}}$ if the equality of 2-morphisms

$$
\omega_{\underline{w}}([\tau] \overline{\mathbf{i}]})=([\overline{\mathbf{i}}] \sigma) \omega_{\underline{. .}}
$$

in TRCAT $_{k}$ holds, i. e. if the diagram

$$
\begin{aligned}
& {[\underline{v}] \overline{[\mathbf{i}]} \stackrel{[\tau] \overline{\mathbf{i}]}}{\longrightarrow}[\underline{w}] \overline{[\mathbf{i}]}} \\
& \omega_{v} \downarrow \sim \quad \omega_{w} \mid \sim
\end{aligned}
$$

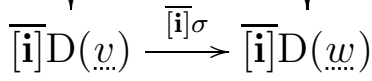


in $\operatorname{TRCAT}\left(\mathrm{D}\left(\mathcal{X}_{1}\right)^{\varepsilon_{1}}, \ldots, \mathrm{D}\left(\mathcal{X}_{n}\right)^{\varepsilon_{n}} ;[\mathbb{I}(\mathcal{Y})]^{\delta}\right)$ is commutative. The following diagram illustrates the situation.

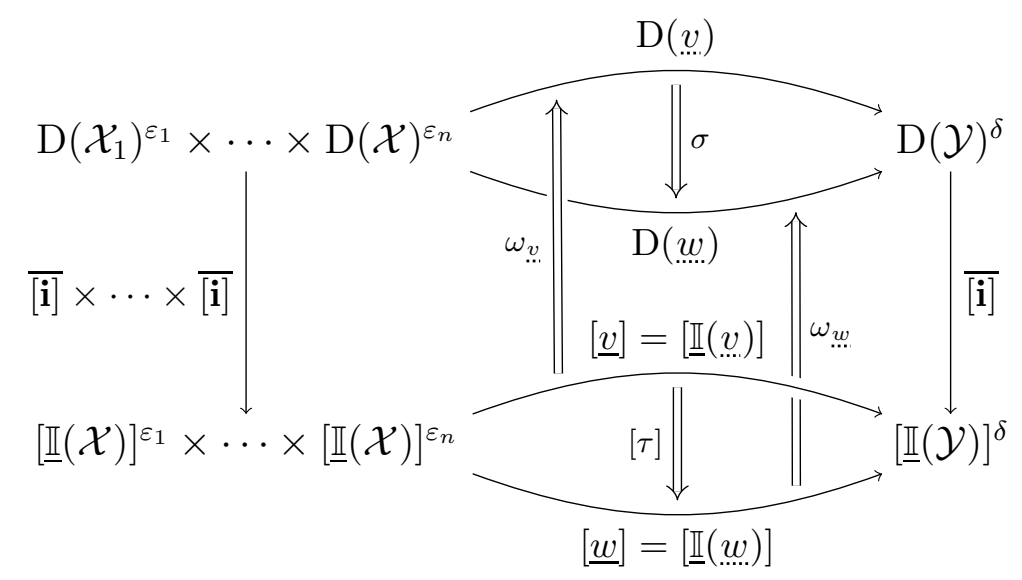

Usually, $\underline{v}$ and $\underline{w}$ are clear from the context and we just say that $\tau$ enhances $\sigma$.

Remark 6.16. Keep the setting of Definition 6.15. Let $u$ be another morphism in $\mathrm{fml}_{\mathrm{k}}^{\prime}$, and assume that $\tau^{\prime}: \underline{w}=\underline{\mathbb{I}}(w) \rightarrow \underline{u}=\underline{\mathbb{I}}(\underline{u})(\underline{w}, \underline{u})$-enhances $\sigma^{\prime}: \mathrm{D}(\underline{w}) \rightarrow \mathrm{D}(\underline{u})$. Then $\tau^{\prime} \tau(\underline{v}, u)$-enhances $\sigma^{\prime} \sigma$. If $\tau(v, w)$-enhances $\sigma$ and both $\tau$ and $\sigma$ are 2-isomorphisms, then $\tau^{-1}(\underline{w}, \underline{v})$-enhances $\sigma^{-1}$.

Proposition 6.17. Let $\alpha:\left(\operatorname{Sh}(\mathcal{Y}), \mathcal{O}_{\mathcal{Y}}\right) \rightarrow\left(\operatorname{Sh}(\mathcal{X}), \mathcal{O}_{\mathcal{X}}\right)$ be a morphism of k-ringed topoi. Then the zig-zag

$$
\underline{\alpha}^{*} \underline{\alpha}_{*}=\mathbf{i} \alpha^{*} \mathbf{e i} \alpha_{*} \stackrel{[\sim]}{\longleftarrow} \mathbf{i} \alpha^{*} \mathbf{e} \alpha_{*} \rightarrow \mathbf{i} \alpha^{*} \alpha_{*} \rightarrow \mathbf{i} \stackrel{[\sim]}{\longleftarrow} \text { id }
$$

of 2-morphisms in $\widetilde{\mathrm{ENH}}_{\mathrm{k}}$ defines a 2-morphism

$$
\underline{\alpha}^{*} \underline{\alpha}_{*} \rightarrow \mathrm{id}
$$

in $\mathrm{ENH}_{\mathrm{k}}$ that enhances the counit $\mathbf{L} \alpha^{*} \mathbf{R} \alpha_{*} \rightarrow$ id (see Remarks 6.18 and 6.19 for more details), and the zig-zag

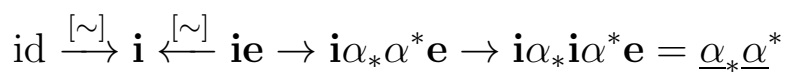

of 2-morphisms in $\widetilde{\mathrm{ENH}}_{\mathrm{k}}$ defines a 2-morphism

$$
\mathrm{id} \rightarrow \underline{\alpha}_{*} \underline{\alpha}^{*}
$$

in $\mathrm{ENH}_{\mathrm{k}}$ that enhances the unit morphism id $\rightarrow \mathbf{R} \alpha_{*} \mathbf{L} \alpha^{*}$.

Remark 6.18. In the formulation of Proposition 6.17 we have implicitly fixed an adjunction $\left(\alpha^{*}, \alpha_{*}\right.$, id $\stackrel{\eta}{\rightarrow} \alpha_{*} \alpha^{*}, \alpha^{*} \alpha_{*} \stackrel{\theta}{\rightarrow}$ id) giving rise to an adjunction $\left(\mathbf{L} \alpha^{*}, \mathbf{R} \alpha_{*}, \eta^{\mathrm{D}}, \theta^{\mathrm{D}}\right)$. We will tacitly fix and use similar data in the following.

Remark 6.19. The precise meaning of the first claim in Proposition 6.17 is: in the zig-zag

$$
\underline{\alpha}^{*} \underline{\alpha}_{*}=\mathbf{i} \alpha^{*} \mathbf{e} \mathbf{i} \alpha_{*} \stackrel{\mathbf{i} \alpha^{*} \mathbf{e} \alpha_{*}}{\longleftarrow} \mathbf{i} \alpha^{*} \mathbf{e} \alpha_{*} \stackrel{\mathbf{i} \alpha^{*} \epsilon \alpha_{*}}{\longrightarrow} \mathbf{i} \alpha^{*} \alpha_{*} \stackrel{\mathbf{i} \theta}{\rightarrow} \mathbf{i} \stackrel{\mathrm{l}}{\leftarrow} \text { id }
$$


of 2-morphisms (between 1-morphisms $\underline{\mathbb{I}}(\mathcal{Y}) \rightarrow \underline{\mathbb{I}}(\mathcal{Y})$ ) in $\widetilde{\mathrm{ENH}}_{\mathrm{k}}$ all left-pointing arrows are objectwise homotopy equivalences (here $\theta$ is as in Remark 6.18); therefore, as explained in Remark 5.9,

$$
\delta(\mathbf{\iota})^{-1} \delta(\mathbf{i} \theta) \delta\left(\mathbf{i} \alpha^{*} \epsilon \alpha_{*}\right) \delta\left(\mathbf{i} \alpha^{*} \mathbf{e} \iota \alpha_{*}\right)^{-1}: \underline{\alpha}^{*} \underline{\alpha}_{*} \rightarrow \mathrm{id}
$$

defines a 2-morphism in $\mathrm{ENH}_{\mathrm{k}}$ (where $\delta: \widetilde{\mathrm{ENH}_{\mathrm{k}}} \rightarrow \mathrm{ENH}_{\mathrm{k}}$ is the functor (5.12)) and this 2-morphism $\left(\alpha^{*} \alpha_{*}\right.$, id)-enhances the counit 2-morphism $\theta^{\mathrm{D}}: \mathbf{L} \alpha^{*} \mathbf{R} \alpha_{*} \rightarrow$ id in $\mathrm{TRCAT}_{\mathrm{k}}$. We leave it to the reader to interpret similar claims accordingly.

Proof. Let $\eta$ and $\theta$ be as in Remark 6.18. The evaluation of (6.41) at $J \in \underline{\mathbb{I}}(\mathcal{Y})$ is the zig-zag

$$
\mathbf{i} \alpha^{*} \mathbf{e i} \alpha_{*} J \stackrel{\mathbf{i} \alpha^{*} \mathbf{e}_{\alpha_{*} J}}{\longleftarrow} \mathbf{i} \alpha^{*} \mathbf{e} \alpha_{*} J \stackrel{\mathbf{i} \alpha^{*} \epsilon_{\alpha_{*} J}}{\longrightarrow} \mathbf{i} \alpha^{*} \alpha_{*} J \stackrel{\mathbf{i} \theta_{J}}{\longrightarrow} \mathbf{i} J \stackrel{\iota_{J}}{\longleftarrow} J
$$

in $\mathbb{I}(\mathcal{Y})$. The two arrows pointing to the left are homotopy equivalences, by Proposition A.2 (and Remark 6.1). This shows that the left-pointing arrows in (6.41) are objectwise homotopy equivalences; hence this zig-zag defines the 2-morphism (6.42). Let us call it $\tau$.

By choosing $\mathbf{R} \alpha_{*}$ and $\mathbf{L} \alpha^{*}$ as in the proof of Proposition 6.5 (more precisely, as in the proof of Proposition 6.8 and as in Remark 6.10) we can assume that $\omega_{\alpha_{*}}$ is the identity and that $\omega_{\alpha^{*}}$ is (6.31). Then the counit $\theta^{\mathrm{D}}: \mathbf{L} \alpha^{*} \mathbf{R} \alpha_{*} \rightarrow$ id is the composition

$$
\begin{aligned}
\left.\theta^{\mathrm{D}}: \mathbf{L} \alpha^{*} \mathbf{R} \alpha_{*}=\overline{\left[\alpha^{*}\right]} \overline{[\mathbf{e}]} q_{\mathcal{X}}\left[\alpha_{*}\right] \overline{\mathbf{i}]}=q_{\mathcal{Y}}\left[\alpha^{*}\right][\mathbf{e}]\left[\alpha_{*}\right] \overline{\mathbf{i}]}\right] \\
\\
\stackrel{q_{\mathcal{Y}}\left[\alpha^{*}\right][\epsilon]\left[\alpha_{*}\right] \overline{\mathbf{i}]}}{\longrightarrow} q_{\mathcal{Y}}\left[\alpha^{*} \alpha_{*}\right] \overline{\mathbf{i}]} \stackrel{q_{\mathcal{Y}}[\theta] \overline{\mathbf{i}]}}{\longrightarrow} q_{\mathcal{Y}} \overline{\mathbf{i}]} \stackrel{\overline{[\ell}^{-1}}{\longrightarrow} \mathrm{id},
\end{aligned}
$$

cf. (6.15). We need to show (cf. (6.38) and Example 6.13) that

$$
[\tau] \overline{[\mathbf{i}]}=\left(\overline{[\mathbf{i}]} \theta^{\mathrm{D}}\right)\left(\omega_{\alpha^{*}} \mathbf{R} \alpha_{*}\right)
$$

as natural transformations $\left[\underline{\alpha}^{*}\right]\left[\underline{\alpha}_{*}\right][\overline{\mathbf{i}}]=\left[\underline{\alpha}^{*}\right] \overline{\mathbf{i}]} \mathbf{R} \alpha_{*} \rightarrow \overline{[\mathbf{i}]}$ between triangulated kfunctors $\mathrm{D}(\mathcal{Y}) \rightarrow[\mathbb{I}(\mathcal{Y})]$. It is enough to show that the evaluations of both transformations at an arbitrary object $F \in \mathrm{D}(\mathcal{Y})$ coincide. Consider the composition

$$
\mathbf{i} \alpha^{*} \mathbf{e i} \alpha_{*} \mathbf{i} F \stackrel{\left[\mathbf{i} \alpha^{*} \mathbf{e}_{\alpha_{*} \mathbf{i} F}\right]^{-1}}{\longrightarrow} \mathbf{i} \alpha^{*} \mathbf{e} \alpha_{*} \mathbf{i} F \stackrel{\left[\mathbf{i} \alpha^{*} \epsilon_{\left.\alpha_{*} \mathbf{i} F\right]}\right.}{\longrightarrow} \mathbf{i} \alpha^{*} \alpha_{*} \mathbf{i} F \stackrel{\left[\mathbf{i} \theta_{\mathbf{i} F]}\right.}{\longrightarrow} \mathbf{i i} F .
$$

If we compose it with $\left[\mathbf{l}_{\mathbf{i} F}\right]^{-1}: \mathbf{i i} F \rightarrow \mathbf{i} F$ we obtain the evaluation of $[\tau][\overline{\mathbf{i}}]$ at $F$; if we compose it with $\left[\mathbf{i}_{F}\right]^{-1}: \mathbf{i i} F \rightarrow \mathbf{i} F$ we obtain the evaluation of $\left(\overline{[\mathbf{i}]} \theta^{\mathrm{D}}\right)\left(\omega_{\alpha^{*}} \mathbf{R} \alpha_{*}\right)$ at $F$ (cf. (6.16)). These two evaluations coincide because $\left[\mathbf{l}_{\mathbf{i} F}\right]=\left[\mathbf{i} \iota_{F}\right]$, by Lemma 6.20 below. This shows that $\underline{\alpha}^{*} \underline{\alpha}_{*} \rightarrow$ id enhances $\mathbf{L} \alpha^{*} \mathbf{R} \alpha_{*} \rightarrow$ id.

The evaluation of the zig-zag (6.43) at $I \in \mathbb{I}(\mathcal{X})$ is the zig-zag

$$
I \stackrel{\iota_{I}}{\longrightarrow} \mathbf{i} I \stackrel{\mathbf{i} \epsilon_{I}}{\longleftarrow} \mathbf{i e} I \stackrel{\mathbf{i} \eta_{\mathrm{e} I}}{\longrightarrow} \mathbf{i} \alpha_{*} \alpha^{*} \mathbf{e} I \stackrel{\mathbf{i} \alpha_{*} \iota_{\alpha *}{ }^{\prime} I}{\longrightarrow} \mathbf{i} \alpha_{*} \mathbf{i} \alpha^{*} \mathbf{e} I=\underline{\alpha}_{*} \underline{\alpha}^{*} I
$$

in $\mathbb{I}(\mathcal{X})$. Its first two arrows are obviously homotopy equivalences. This shows that the indicated two arrows in (6.43) are objectwise homotopy equivalences; hence this zig-zag defines the 2-morphism (6.44). Let us call it $\rho$. 
We choose $\mathbf{R} \alpha^{*}$ and $\mathbf{L} \alpha^{*}$ as before. Consider the composition

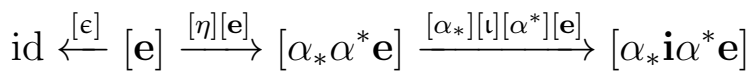

of transformations of endofunctors of $[\underline{\mathrm{C}}(\mathcal{X})]$. If we apply $q_{\mathcal{X}}$, the left-pointing arrow becomes invertible and we obtain a transformation

$$
\tilde{\eta}: q_{\mathcal{X}} \rightarrow q_{X}\left[\alpha_{*}\right][\mathbf{i}]\left[\alpha^{*}\right][\mathbf{e}]=q_{X}\left[\alpha_{*}\right] \overline{\mathbf{i}]} q_{\mathcal{Y}}\left[\alpha^{*}\right][\mathbf{e}]=\mathbf{R} \alpha_{*} \overline{\left[\alpha^{*}\right]} \overline{[\mathbf{e}]} q_{\mathcal{X}}=\mathbf{R} \alpha_{*} \mathbf{L} \alpha^{*} q_{\mathcal{X}}
$$

using (6.15). It is easy to see (using that $\left[\alpha_{*} \mathbf{i} \alpha^{*} \mathbf{e}\right]$ preserves quasi-isomorphisms) that there is a unique transformation $\eta^{\mathrm{D}}:$ id $\rightarrow \mathbf{R} \alpha_{*} \mathbf{L} \alpha^{*}$ such that $\eta^{\mathrm{D}} q_{\mathcal{X}}=\tilde{\eta}$; moreover, this transformation is the unit of the adjunction $\left(\mathbf{L} \alpha^{*}, \mathbf{R} \alpha_{*}\right)$.

We need to show (cf. (6.38) and Example 6.13) that $\left(\left[\underline{\alpha}_{*}\right] \omega_{\alpha^{*}}\right)([\rho][\overline{\mathbf{i}}])=\overline{[\mathbf{i}]} \eta^{\mathrm{D}}$. By precomposing with $q_{\mathcal{X}}$ it is enough to show that

$$
\left(\left[\underline{\alpha}_{*}\right] \omega_{\alpha^{*}} q_{\mathcal{X}}\right)([\rho][\mathbf{i}])=\overline{[\mathbf{i}]} \tilde{\eta}
$$

as natural transformations $[\mathbf{i}] \rightarrow \overline{[\mathbf{i}]} \mathbf{R} \alpha_{*} \mathbf{L} \alpha^{*} q_{\mathcal{X}}=[\mathbf{i}]\left[\alpha_{*}\right][\mathbf{i}]\left[\alpha^{*}\right][\mathbf{e}]$ between triangulated functors $[\underline{\mathrm{C}}(\mathcal{X})] \rightarrow[\underline{\mathbb{I}}(\mathcal{X})]$. The evaluation of $\overline{[\mathbf{i}} \tilde{\eta}$ at $E \in[\underline{\mathrm{C}}(\mathcal{X})]$ is

$$
\mathbf{i} E \stackrel{\left[\mathbf{i} \epsilon_{E}\right]^{-1}}{\longrightarrow} \mathbf{i e} E \stackrel{\left[\mathbf{i} \eta_{\mathbf{e} E}\right]}{\longrightarrow} \mathbf{i} \alpha_{*} \alpha^{*} \mathbf{e} E \stackrel{\left[\mathbf{i} \alpha_{*} \mathbf{l}_{\alpha^{*} \mathbf{e} E}\right]}{\longrightarrow} \mathbf{i} \alpha_{*} \mathbf{i} \alpha^{*} \mathbf{e} E .
$$

On the other hand, the evaluation of $\left(\left[\underline{\alpha}_{*}\right] \omega_{\alpha^{*}} q_{\mathcal{X}}\right)([\rho][\mathbf{i}])$ at $E$ is (the first four arrows are obtained from $(6.50)$ and compose to $([\rho][\mathbf{i}])_{E}$, the last arrow is $\left(\left[\underline{\alpha}_{*}\right] \omega_{\alpha^{*}}\right)_{E}$, cf. $(6.16)$ or $(6.31))$

$\mathbf{i} E \stackrel{\left[\mathbf{l i}_{E}\right]}{\longrightarrow} \mathbf{i i} E \stackrel{\left[\mathbf{i} \epsilon_{\mathbf{i} E}\right]^{-1}}{\longrightarrow} \mathbf{i e i} E \stackrel{\left[\mathbf{i} \eta_{\mathbf{e i} E]}\right.}{\longrightarrow} \mathbf{i} \alpha_{*} \alpha^{*} \mathbf{e i} E \stackrel{\left[\mathbf{i} \alpha_{*} \mathbf{l}_{\alpha^{*}} \mathbf{e} E\right]}{\longrightarrow} \mathbf{i} \alpha_{*} \mathbf{i} \alpha^{*} \mathbf{e i} E \stackrel{\left[\mathbf{i} \alpha_{*} \mathbf{i} \alpha^{*} \mathbf{e} \iota_{E}\right]^{-1}}{\longrightarrow} \mathbf{i} \alpha_{*} \mathbf{i} \alpha^{*} \mathbf{e} E$

To see that the compositions in (6.54) and (6.55) coincide consider the commutative diagram

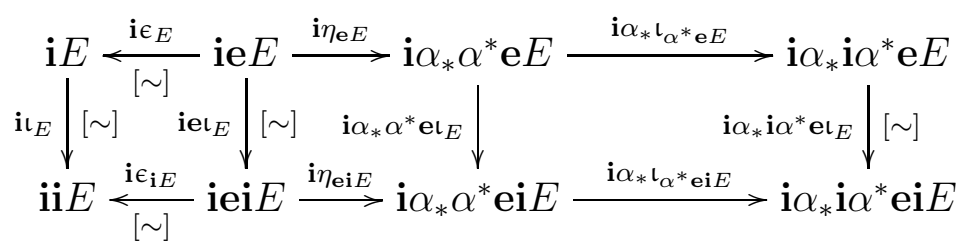

in $\mathbb{I}(\mathcal{X})$, note that the arrows labeled $[\sim]$ become invertible in $[\mathbb{I}(\mathcal{X})]$, and that moreover $\left[\mathbf{i} \iota_{E}\right]=\left[\mathbf{l}_{\mathbf{i} E}\right]$ by Lemma 6.20 below. This shows that id $\rightarrow \underline{\alpha}_{*} \underline{\alpha}^{*}$ enhances id $\rightarrow \mathbf{R} \alpha_{*} \mathbf{L} \alpha^{*}$.

Lemma 6.20. Let $\mathcal{X}$ be a $\mathrm{k}$-ringed site and $F \in \underline{\mathrm{C}}(\mathcal{X})$. Then the two morphisms $\left[\boldsymbol{\iota}_{\mathbf{i} F}\right],\left[\mathbf{i} \iota_{F}\right]: \mathbf{i} F \rightarrow \mathbf{i i} F$ in $[\underline{\mathrm{C}}(\mathcal{X})]$ coincide, i. e. $\left[\boldsymbol{\iota}_{\mathbf{i} F}\right]=\left[\mathbf{i} \iota_{F}\right]$. 
Proof. Consider the commutative square

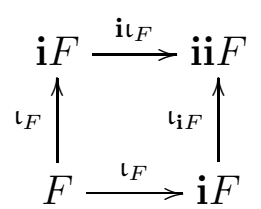

in $\mathrm{C}(\mathcal{X})$. Since $\mathfrak{t}_{F}$ is a quasi-isomorphism and $\mathbf{i i} F$ is h-injective, the map

$$
\left[\mathfrak{\iota}_{F}\right]^{*}:\left[\underline{\mathrm{C}}_{\mathcal{Y}}\right](\mathbf{i} F, \mathbf{i i} F) \rightarrow\left[\underline{\mathrm{C}}_{\mathcal{Y}}\right](F, \mathbf{i i} F)
$$

is an isomorphism; it maps $\left[\mathfrak{l}_{\mathbf{i} F}\right]$ and $\left[\mathbf{i}_{F}\right]$ to the same element.

Lemma 6.21. Let $\left(\operatorname{Sh}(\mathcal{Z}), \mathcal{O}_{\mathcal{Z}}\right) \stackrel{\beta}{\rightarrow}\left(\operatorname{Sh}(\mathcal{Y}), \mathcal{O}_{\mathcal{Y}}\right) \stackrel{\alpha}{\rightarrow}\left(\operatorname{Sh}(\mathcal{X}), \mathcal{O}_{\mathcal{X}}\right)$ be morphisms of $\mathrm{k}$-ringed topoi. Then the zig-zags

$$
\begin{aligned}
\underline{\mathrm{id}}_{*} & =\mathbf{i} \stackrel{[\sim]}{\longleftarrow} \mathrm{id}, \\
\underline{(\alpha \beta)}_{*} & =\mathbf{i}(\alpha \beta)_{*} \stackrel{\sim}{\rightarrow} \mathbf{i} \alpha_{*} \beta_{*} \stackrel{[\sim]}{\longrightarrow} \mathbf{i} \alpha_{*} \mathbf{i} \beta_{*}=\underline{\alpha}_{*} \underline{\beta}_{*}, \\
\underline{\mathrm{id}}^{*} & =\mathbf{i e} \stackrel{[\sim]}{\longrightarrow} \mathbf{i} \stackrel{[\sim]}{\longleftarrow} \mathrm{id}, \\
{\underline{(\alpha \beta)^{*}}}^{*} & =\mathbf{i}(\alpha \beta)^{*} \mathbf{e} \stackrel{\sim}{\rightarrow} \mathbf{i} \beta^{*} \alpha^{*} \mathbf{e} \stackrel{[\sim]}{\longleftarrow} \mathbf{i} \beta^{*} \mathbf{e} \alpha^{*} \mathbf{e} \stackrel{[\sim]}{\longrightarrow} \mathbf{i} \beta^{*} \mathbf{e i} \alpha^{*} \mathbf{e}=\underline{\beta}^{*} \underline{\alpha}^{*}
\end{aligned}
$$

of objectwise homotopy equivalences in $\widetilde{\mathrm{ENH}}_{\mathrm{k}}$ define 2-isomorphisms

$$
\begin{aligned}
\underline{\mathrm{id}}_{*} & \stackrel{\sim}{\rightarrow} \mathrm{id}, \\
\underline{(\alpha \beta)} & \stackrel{\sim}{\rightarrow} \underline{\alpha}_{*} \underline{\beta}_{*}, \\
\underline{\mathrm{id}}^{*} & \sim \\
\underline{(\alpha \beta)} & \stackrel{\mathrm{id},}{\leftarrow} \underline{\beta}^{*} \underline{\alpha}^{*}
\end{aligned}
$$

in $\mathrm{ENH}_{\mathrm{k}}$ that enhance the isomorphisms $\mathbf{R i d}_{*} \cong \mathrm{id}, \mathbf{R}(\alpha \beta)_{*} \cong \mathbf{R} \alpha_{*} \mathbf{R} \beta_{*}, \mathbf{L i d}^{*} \cong \mathrm{id}$, $\mathbf{L}(\alpha \beta)^{*} \cong \mathbf{L} \beta^{*} \mathbf{L} \alpha^{*}$.

In the formulation of the lemma, we use the usual isomorphisms $(\alpha \beta)_{*} \stackrel{\sim}{\rightarrow} \alpha_{*} \beta_{*}$ and $(\alpha \beta)^{*} \stackrel{\sim}{\rightarrow} \beta^{*} \alpha^{*}$, cf. Remark 6.18.

Proof. Let $J \in \mathbb{I}(\mathcal{Z})$. Obviously, $J \stackrel{\iota_{J}}{\rightarrow} \mathbf{i} J=\underline{\mathrm{id}}_{*} J$ is a homotopy equivalence. Proposition A.9 shows that $\beta_{*} J \stackrel{\mathfrak{L}_{* * J}}{\longrightarrow} \mathbf{i} \beta_{*} J$ is a quasi-isomorphism between weakly h-injective complexes and that

$$
\stackrel{(\alpha \beta)}{*}_{*} J=\mathbf{i}(\alpha \beta)_{*} J \stackrel{\sim}{\rightarrow} \mathbf{i} \alpha_{*} \beta_{*} J \stackrel{\mathbf{i} \alpha_{*} \mathfrak{l}_{*} J}{\longrightarrow} \mathbf{i} \alpha_{*} \mathbf{i} \beta_{*} J=\underline{\alpha}_{*} \underline{\beta}_{*} J
$$

is a homotopy equivalence.

Let $I \in \mathbb{I}(\mathcal{X})$. Obviously, $I \stackrel{\mathfrak{L}_{I}}{\rightarrow} \mathbf{i} I \stackrel{\mathrm{i} \epsilon_{I}}{\longleftarrow} \mathbf{i e} I=\underline{\mathrm{id}}^{*} I$ consists of homotopy equivalences. Proposition A.2 shows that $F:=\alpha^{*} \mathbf{e} I$ is h-flat, so both morphisms

$$
F \stackrel{\epsilon_{F}}{\longleftarrow} \mathbf{e} F \stackrel{\mathbf{e}_{F}}{\longrightarrow} \mathbf{e i} F
$$


are quasi-isomorphisms between h-flat complexes, and that $\mathbf{i} \beta^{*}$ maps them to homotopy equivalences. We obtain homotopy equivalences

$$
{\underline{(\alpha \beta)^{*}}}^{*}=\mathbf{i}(\alpha \beta)^{*} \mathbf{e} I \stackrel{\sim}{\rightarrow} \mathbf{i} \beta^{*} \alpha^{*} \mathbf{e} I=\mathbf{i} \beta^{*} F \stackrel{\mathbf{i} \beta^{*} \epsilon_{F}}{\longleftarrow} \mathbf{i} \beta^{*} \mathbf{e} F \stackrel{\mathbf{i} \beta^{*} \mathbf{e}_{F}}{\longrightarrow} \mathbf{i} \beta^{*} \mathbf{e i} F=\underline{\beta}^{*} \underline{\alpha}^{*} I .
$$

We leave the rest of the proof to the reader.

The following result uses fixed data turning $\left(\mathrm{C}(\mathcal{X}), \otimes, \mathcal{O}_{\mathcal{X}}\right)$ into a symmetric monoidal category.

Lemma 6.22. Let $(\mathcal{X}, \mathcal{O})$ be a $\mathrm{k}$-ringed site. Then the zig-zags

$$
\begin{aligned}
& (\underline{\mathcal{O}} \underline{\otimes}-)=\mathbf{i}(\mathbf{e i O} \otimes \mathbf{e}(-)) \stackrel{[\sim]}{\stackrel{[}{\longleftarrow}} \mathbf{i}(\mathbf{e O} \otimes \mathbf{e}(-)) \stackrel{[\sim]}{\longrightarrow} \mathbf{i}(\mathcal{O} \otimes \mathbf{e}(-)) \stackrel{\sim}{\rightarrow} \mathbf{i e} \stackrel{[\sim]}{\longrightarrow} \mathbf{i} \stackrel{[\sim]}{\longleftarrow} \mathbf{i d}, \\
& (-\underline{\otimes} \underline{\mathcal{O}})=\mathbf{i}(\mathbf{e}(-) \otimes \mathbf{e i O}) \stackrel{[\sim]}{\stackrel{[}{\longleftarrow}} \mathbf{i}(\mathbf{e}(-) \otimes \mathbf{e O}) \stackrel{[\sim]}{\longrightarrow} \mathbf{i}(\mathbf{e}(-) \otimes \mathcal{O}) \stackrel{\sim}{\rightarrow} \mathbf{i e} \stackrel{[\sim]}{\longrightarrow} \mathbf{i} \stackrel{[\sim]}{\longleftarrow} \mathbf{i d}, \\
& ((-\underline{\otimes}-) \underline{\otimes}-)=\mathbf{i}(\mathbf{e i}(\mathbf{e}(-) \otimes \mathbf{e}(-)) \otimes \mathbf{e}(-)) \stackrel{[\sim]}{\longleftarrow} \mathbf{i}(\mathbf{e}(\mathbf{e}(-) \otimes \mathbf{e}(-)) \otimes \mathbf{e}(-)) \\
& \quad \stackrel{[\sim]}{\longrightarrow} \mathbf{i}((\mathbf{e}(-) \otimes \mathbf{e}(-)) \otimes \mathbf{e}(-)) \stackrel{\sim}{\rightarrow} \mathbf{i}(\mathbf{e}(-) \otimes(\mathbf{e}(-) \otimes \mathbf{e}(-))) \stackrel{[\sim]}{\longleftarrow} \ldots \stackrel{[\sim]}{\longrightarrow}(-\underline{\otimes}(-\underline{\otimes}-)), \\
& (-\underline{\otimes} ?)=\mathbf{i}(\mathbf{e}(-) \otimes \mathbf{e}(?)) \stackrel{\sim}{\rightarrow} \mathbf{i}(\mathbf{e}(?) \otimes \mathbf{e}(-))=(? \underline{\otimes}-)
\end{aligned}
$$

of objectwise homotopy equivalences in $\widetilde{\mathrm{ENH}}_{\mathrm{k}}$ define 2-isomorphisms

$$
\begin{aligned}
(\underline{\mathcal{O}} \underline{\otimes}-) & \stackrel{\sim}{\rightarrow} \mathrm{id}, \\
(-\underline{\otimes} \underline{\mathcal{O}}) & \stackrel{\sim}{\rightarrow} \mathrm{id}, \\
((-\underline{\otimes}-) \underline{\otimes}-) & \stackrel{\sim}{\rightarrow}(-\underline{\otimes}(-\underline{\otimes}-)), \\
(-\underline{\otimes} ?) & \stackrel{\sim}{\rightarrow}(? \underline{\underline{\theta}}-),
\end{aligned}
$$

that enhance the corresponding isomorphisms $\left(\mathcal{O} \otimes^{\mathbf{L}}-\right) \stackrel{\sim}{\rightarrow} \mathrm{id},\left(-\otimes^{\mathbf{L}} \mathcal{O}\right) \stackrel{\sim}{\rightarrow} \mathrm{id}$, $\left(\left(-\otimes^{\mathbf{L}}-\right) \otimes^{\mathbf{L}}-\right) \stackrel{\sim}{\rightarrow}\left(-\otimes^{\mathbf{L}}\left(-\otimes^{\mathbf{L}}-\right)\right),\left(-\otimes^{\mathbf{L}} ?\right) \stackrel{\sim}{\rightarrow}\left(? \otimes^{\mathbf{L}}-\right)$.

Proof. Obvious and left to the reader.

Lemma 6.23. Let $(\mathcal{X}, \mathcal{O})$ be a k-ringed site. Then the 2-morphism

$$
\underline{\operatorname{Hom}}(-,-)=\Gamma \mathcal{H o m} \rightarrow \Gamma \mathbf{i} \mathcal{H o m}(-,-)=\underline{\Gamma} \underline{\mathcal{H} o m}(-,-)
$$

in $\widetilde{\mathrm{ENH}}_{\mathrm{k}}$ is an objectwise homotopy equivalence and the induced isomorphism

$$
\underline{\operatorname{Hom}}(-,-) \stackrel{\sim}{\rightarrow} \underline{\Gamma} \underline{\mathcal{H} o m}(-,-)
$$

in $\mathrm{ENH}_{\mathrm{k}}$ enhances $\mathbf{R H o m}(-,-) \stackrel{\sim}{\rightarrow} \mathbf{R} \Gamma \mathbf{R H o m}(-,-)$.

Proof. For $I, J \in \mathbb{I}(\mathcal{X})$ the morphism $\mathcal{H o m}(I, J) \rightarrow \mathbf{i} \mathcal{H o m}(I, J)$ is a quasi-isomorphism between weakly h-injective complexes, by Proposition A.6, so that we can apply Proposition A.9. The rest of the proof is left to the reader. 
Lemma 6.24. Let $\alpha:\left(\operatorname{Sh}(\mathcal{Y}), \mathcal{O}_{\mathcal{Y}}\right) \rightarrow\left(\operatorname{Sh}(\mathcal{X}), \mathcal{O}_{\mathcal{X}}\right)$ be a morphism of $\mathrm{k}$-ringed topoi. Then the zig-zag

$$
\begin{aligned}
\underline{\alpha}^{*}(-\underline{\otimes}-)=\mathbf{i} \alpha^{*} \mathbf{e i}(\mathbf{e}(-) \otimes \mathbf{e}(-)) \stackrel{[\sim]}{\stackrel{\mathbf{i}}{*} \alpha^{*} \mathbf{e}(\mathbf{e}(-) \otimes \mathbf{e}(-)) \stackrel{[\sim]}{\longrightarrow} \mathbf{i} \alpha^{*}(\mathbf{e}(-) \otimes \mathbf{e}(-))} \\
\stackrel{\sim}{\rightarrow} \mathbf{i}\left(\alpha^{*} \mathbf{e}(-) \otimes \alpha^{*} \mathbf{e}(-)\right) \stackrel{[\sim]}{\longleftarrow} \mathbf{i}\left(\mathbf{e} \alpha^{*} \mathbf{e}(-) \otimes \mathbf{e} \alpha^{*} \mathbf{e}(-)\right) \\
\left.\stackrel{[\sim]}{\longrightarrow} \mathbf{i}\left(\mathbf{e i} \alpha^{*} \mathbf{e}(-) \otimes \mathbf{e i} \alpha^{*} \mathbf{e}(-)\right)=\left(\underline{\alpha}^{*}-\right) \underline{\otimes}\left(\underline{\alpha}^{*}-\right)\right)
\end{aligned}
$$

of objectwise homotopy equivalences in $\widetilde{\mathrm{ENH}}_{\mathrm{k}}$ defines a 2-isomorphism

$$
\underline{\alpha}^{*}(-\underline{\otimes}-) \stackrel{\sim}{\rightarrow}\left(\underline{\alpha}^{*}-\right) \underline{\otimes}\left(\underline{\alpha}^{*}-\right)
$$

in $\mathrm{ENH}_{\mathrm{k}}$ that enhances $\mathbf{L} \alpha^{*}\left(-\otimes^{\mathbf{L}}-\right) \stackrel{\sim}{\rightarrow} \mathbf{L} \alpha^{*}(-) \otimes^{\mathbf{L}} \mathbf{L} \alpha^{*}(-)$ (cf. [Lip09, Prop. 3.2.4.(i)] for ringed spaces).

Proof. The proof uses Proposition A.2 and is left to the reader.

Proposition 6.25. Let $\alpha:\left(\operatorname{Sh}(\mathcal{Y}), \mathcal{O}_{\mathcal{Y}}\right) \rightarrow\left(\operatorname{Sh}(\mathcal{X}), \mathcal{O}_{\mathcal{X}}\right)$ be a morphism of $\mathrm{k}$-ringed topoi. Then the zig-zag

$$
\begin{array}{r}
\underline{\alpha}_{*} \underline{\mathcal{H o m}}\left(\underline{\alpha}^{*}-,-\right)=\mathbf{i} \alpha_{*} \mathbf{i} \mathcal{H} \operatorname{Hom}\left(\mathbf{i} \alpha^{*} \mathbf{e}(-),-\right) \stackrel{[\sim]}{\longleftarrow} \mathbf{i} \alpha_{*} \mathcal{H o m}\left(\mathbf{i} \alpha^{*} \mathbf{e}(-),-\right) \\
\stackrel{[\sim]}{\longrightarrow} \mathbf{i} \alpha_{*} \mathcal{H o m}\left(\alpha^{*} \mathbf{e}(-),-\right) \stackrel{\sim}{\rightarrow} \mathbf{i} \mathcal{H o m}\left(\mathbf{e}(-), \alpha_{*}(-)\right) \stackrel{[\sim]}{\longrightarrow} \mathbf{i} \mathcal{H o m}\left(\mathbf{e}(-), \mathbf{i} \alpha_{*}(-)\right) \\
\stackrel{[\sim]}{\longleftarrow} \mathbf{i} \mathcal{H o m}\left(-, \mathbf{i} \alpha_{*}(-)\right)=\underline{\mathcal{H o m}}\left(-, \underline{\alpha}_{*}-\right)
\end{array}
$$

of objectwise homotopy equivalences in $\widetilde{\mathrm{ENH}}_{\mathrm{k}}$ defines a 2-isomorphism

$$
\underline{\alpha}_{*} \underline{\mathcal{H o m}}\left(\underline{\alpha}^{*}-,-\right) \stackrel{\sim}{\rightarrow} \underline{\mathcal{H} o m}\left(-, \underline{\alpha}_{*}-\right)
$$

in $\mathrm{ENH}_{\mathrm{k}}$ that enhances $\mathbf{R} \alpha_{*} \mathbf{R} \mathcal{H o m}\left(\mathbf{L} \alpha^{*}-,-\right) \stackrel{\sim}{\rightarrow} \mathbf{R} \mathcal{H o m}\left(-, \mathbf{R} \alpha_{*}-\right)$.

Proof. Let $I \in \mathbb{I}(\mathcal{X})$ and $J \in \mathbb{I}(\mathcal{Y})$ and evaluate the zig-zag $(6.77)$ at $(I, J)$. Then the first arrow is a homotopy equivalence because $L:=\mathcal{H o m}\left(\mathbf{i} \alpha^{*} \mathbf{e} I, J\right)$ is weakly hinjective by Proposition A.6, so that we can apply Proposition A.9 to $L \stackrel{\text { ` }}{\rightarrow} \mathbf{i} L$. The second arrow is a homotopy equivalence because $\mathcal{H o m}\left(\mathbf{i} \alpha^{*} \mathbf{e} I, J\right) \rightarrow \mathcal{H o m}\left(\alpha^{*} \mathbf{e} I, J\right)$ is a quasi-isomorphism between weakly h-injective complexes, by Propositions A.5.(a) and Proposition A.6, so that we can apply Proposition A.9. The third arrow comes from the usual isomorphism. The fourth arrow is a homotopy equivalence by Proposition A.9 and Corollary A.12. Proposition A.5.(a) shows that the last arrow is a homotopy equivalence. The rest of the proof is left to the reader. 
Proposition 6.26. Let $\left(\mathcal{X}, \mathcal{O}_{\mathcal{X}}\right)$ be a k-ringed site. Then the zig-zag

$$
\begin{aligned}
\underline{\mathcal{H} o m}(-\underline{\otimes}-,-)=\mathbf{i} \mathcal{H o m}(\mathbf{i}(\mathbf{e}(-) \otimes \mathbf{e}(-)),-) \\
\stackrel{[\sim]}{\longrightarrow} \mathbf{i} \mathcal{H o m}(\mathbf{e}(-) \otimes \mathbf{e}(-),-) \stackrel{[\sim]}{\longleftarrow} \mathbf{i} \mathcal{H o m}(\mathbf{e}(-) \otimes-,-) \\
\stackrel{\sim}{\rightarrow} \mathbf{i} \mathcal{H o m}(\mathbf{e}(-), \mathcal{H o m}(-,-)) \stackrel{[\sim]}{\longrightarrow} \mathbf{i} \mathcal{H} \operatorname{Hom}(\mathbf{e}(-), \mathbf{i} \mathcal{H o m}(-,-)) \\
\stackrel{[\sim]}{\longleftarrow} \mathbf{i} \mathcal{H o m}(-, \mathbf{i} \mathcal{H o m}(-,-))=\underline{\mathcal{H o m}}(-, \underline{\mathcal{H} o m}(-,-))
\end{aligned}
$$

of objectwise homotopy equivalences in $\widetilde{\mathrm{ENH}}_{\mathrm{k}}$ defines a 2-isomorphism

$$
\underline{\mathcal{H o m}}(-\underline{\otimes}-,-) \stackrel{\sim}{\rightarrow} \underline{\mathcal{H} o m}(-, \underline{\mathcal{H} o m}(-,-))
$$

in $\mathrm{ENH}_{\mathrm{k}}$ that enhances $\mathbf{R} \mathcal{H o m}\left(-\otimes^{\mathbf{L}}-,-\right) \stackrel{\sim}{\rightarrow} \mathbf{R} \mathcal{H o m}(-, \mathbf{R} \mathcal{H o m}(-,-))$.

Proof. Let $I, J, L \in \underline{\mathbb{I}}(\mathcal{X})$ and evaluate the zig-zag (6.79) at $(I, J, L)$. The first two arrows are homotopy equivalences by Proposition A.5.(a) and h-flatness of $\mathbf{e} I$, the third arrow is the usual isomorphism, the fourth arrow is a homotopy equivalence by Proposition A.6 and Corollary A.12, and the fifth arrow is a homotopy equivalence by Proposition A.5.(a). We leave the rest of the proof to the reader.

6.5.2. Lifts of commutative diagrams. We indicate some relations between the 2morphisms we have constructed so far.

Proposition 6.27. Let $\alpha:\left(\operatorname{Sh}(\mathcal{Y}), \mathcal{O}_{\mathcal{Y}}\right) \rightarrow\left(\operatorname{Sh}(\mathcal{X}), \mathcal{O}_{\mathcal{X}}\right)$ be a morphism of k-ringed topoi. Then the two 1-morphisms $\underline{\alpha}^{*}$ and $\underline{\alpha}_{*}$ and the two 2-morphisms (6.44) and (6.42) form an adjunction in $\mathrm{ENH}_{\mathrm{k}}$, i. e. the two diagrams in $\mathrm{ENH}_{\mathrm{k}}$ in (1.3) commute.

Proof. Let $\underline{\eta}$ : id $\rightarrow \underline{\alpha}_{*} \underline{\alpha}^{*}$ and $\underline{\theta}: \underline{\alpha}^{*} \underline{\alpha}_{*} \rightarrow$ id denote (6.44) and (6.42), respectively. We claim that the composition

$$
\underline{\alpha}_{*} \stackrel{\eta \underline{\underline{\alpha}}}{\longrightarrow} \underline{\alpha}_{*} \underline{\alpha}^{*} \underline{\alpha}_{*} \stackrel{\underline{\alpha}_{*} \underline{\theta}}{\longrightarrow} \underline{\alpha}_{*}
$$

of 2-morphisms in $\mathrm{ENH}_{\mathrm{k}}$ is the identity 2-morphism. 
Let $J \in \underline{\mathbb{I}}(\mathcal{Y})$ and consider the commutative diagram

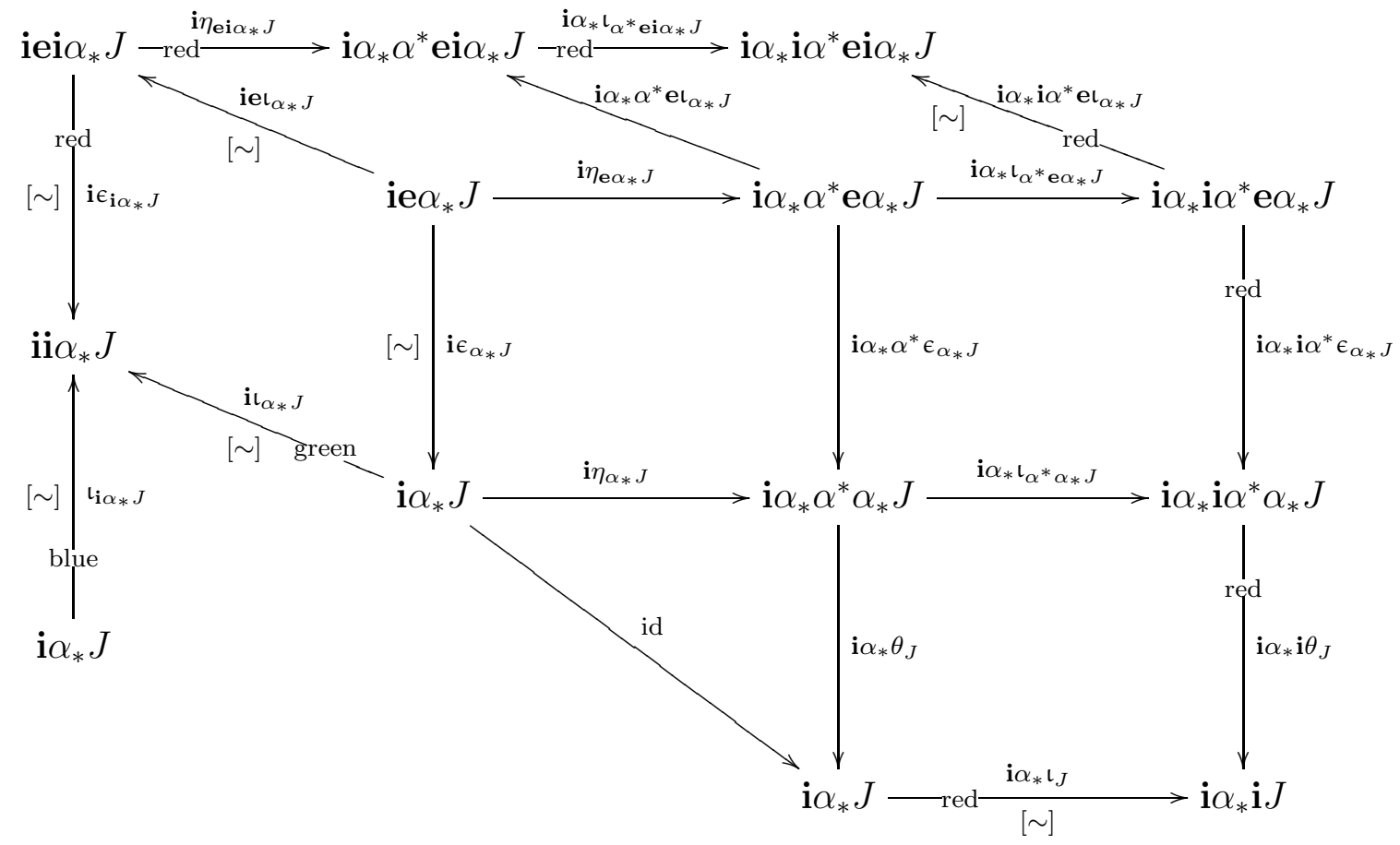

in $\mathbb{I}(\mathcal{X})$; the arrows labeled $[\sim]$ become invertible in $[\mathbb{I}(\mathcal{X})]$. We view $J$ as a variable in the rest of this proof. Then our diagram is a commutative diagram in $\widetilde{\mathrm{ENH}}_{\mathrm{k}}(\underline{\mathbb{I}}(\mathcal{Y}) ; \underline{\mathbb{I}}(\mathcal{X}))$ and all arrows labeled $[\sim]$ are objectwise homotopy equivalences. If we pass to $\mathrm{ENH}_{\mathrm{k}}$, these labeled arrows become invertible, and we define $\beta$ (resp. $\gamma$ ) to be the composition of the path starting with the blue (resp. green) arrow and then following the red arrows and the inverses of the red arrows. Then $\beta$ is the 2morphism (6.81) in $\mathrm{ENH}_{\mathrm{k}}$, cf. (6.47), (6.50). Commutativity of the diagram obviously shows that $\gamma$ is the identity 2-morphism in $\mathrm{ENH}_{\mathrm{k}}$. Proposition 5.10, more precisely (5.24), shows that $\delta\left(\mathbf{i} \iota \alpha_{*}\right)=\delta\left(\mathbf{i} \alpha_{*}\right)$ in $\mathrm{ENH}_{\mathrm{k}}$, i. e. blue and green arrow are equal in $\mathrm{ENH}_{\mathrm{k}}$, and our claim follows.

Similarly, one shows that the composition $\underline{\alpha}^{*} \stackrel{\underline{\alpha}^{*} \eta}{\longrightarrow} \underline{\alpha}^{*} \underline{\alpha}_{*} \underline{\alpha}^{*} \stackrel{\underline{\theta} \underline{\alpha}^{*}}{\longrightarrow} \underline{\alpha}^{*}$ is the identity. This shows that the two diagrams in (1.3) commute.

In the following result we use results and terminology from [ML98, IV.7], [Lip09, 3.3 , generalized to 2-categories in the obvious way (see also Remark 7.24).

Proposition 6.28. Let $\left(\operatorname{Sh}(\mathcal{Z}), \mathcal{O}_{\mathcal{Z}}\right) \stackrel{\beta}{\rightarrow}\left(\operatorname{Sh}(\mathcal{Y}), \mathcal{O}_{\mathcal{Y}}\right) \stackrel{\alpha}{\rightarrow}\left(\operatorname{Sh}(\mathcal{X}), \mathcal{O}_{\mathcal{X}}\right)$ be morphisms of k-ringed topoi. Then the 2-isomorphisms $\underline{\mathrm{id}}_{*} \stackrel{(6.63)}{\sim} \mathrm{id}$ and $\mathrm{id} \stackrel{(6.65)}{\sim} \underline{\mathrm{id}}^{*}$ are conjugate and so are the 2-isomorphisms $\underline{(\alpha \beta)}_{*} \stackrel{(6.64)}{\sim} \underline{\alpha}_{*} \underline{\beta}_{*}$ and $\underline{\beta}^{*} \underline{\alpha}^{*} \stackrel{(6.66)}{\sim} \underline{(\alpha \beta)^{*}}$, i. e. the diagrams (1.8) and (1.7) commute. Here we use the two adjunctions $\left(\underline{\mathrm{id}}^{*}, \underline{\mathrm{id}}_{*}\right)$ 
and (id, id), and the two adjunctions $\left(\underline{(\alpha \beta)^{*}}, \underline{(\alpha \beta)_{*}}\right)$ and $\left(\underline{\beta}^{*} \underline{\alpha}^{*}, \underline{\alpha}_{*} \underline{\beta}_{*}\right)$ obtained from Proposition 6.27.

Proof. We only show the second claim and leave the first to the reader. We need to show that the diagram

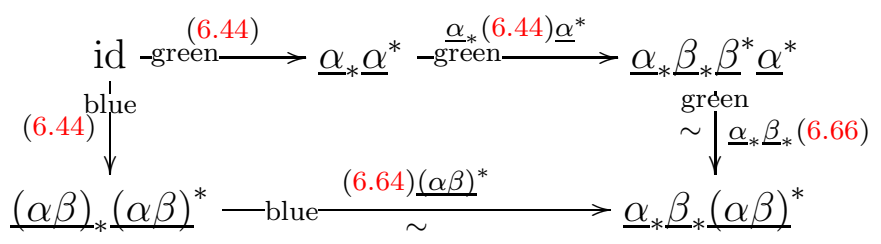

of 2-morphisms in $\mathrm{ENH}_{\mathrm{k}}$ commutes, cf. [Lip09, 3.6.2)]. Replacing the arrows in this diagram by their defining zig-zags of 2-morphisms in $\widetilde{\mathrm{ENH}}_{\mathrm{k}}$ yields the outer arrows in diagram (6.84) on page 63 where $I \in \mathbb{I}(\mathcal{X})$; this diagram lives in $\mathbb{I}(\mathcal{X})$ and is commutative if we remove the bent green arrow; here $\eta, \eta^{\prime}$ and $\eta^{\prime \prime}$ denote units of the tacitly fixed adjunctions $\left(\alpha^{*}, \alpha_{*}\right),\left(\beta^{*}, \beta_{*}\right)$ and $\left((\alpha \beta)^{*},(\alpha \beta)_{*}\right)$, and of course we have tacitly assumed that the isomorphisms $\sigma:(\alpha \beta)_{*} \stackrel{\sim}{\rightarrow} \alpha_{*} \beta_{*}$ and $\tau: \beta^{*} \alpha^{*} \rightarrow(\alpha \beta)^{*}$ used to construct (6.64) and (6.66) are conjugate (this implies that the "pentagon" in diagram (6.84) commutes); the arrows labeled $[\sim]$ become invertible in $[\mathbb{I}(\mathcal{X})]$. In the rest of this proof we view $I$ as a variable. Then diagram (6.84) is a commutative diagram in $\widehat{\mathrm{ENH}}_{\mathrm{k}}(\underline{\mathbb{I}}(\mathcal{X}), \underline{\mathbb{I}}(\mathcal{X}))$ if we forget the bent green arrow, and the arrows labeled $[\sim]$ are objectwise homotopy equivalences. Its red and blue (resp. red and green) arrows give rise to the blue (resp. green) arrows in (6.83). Proposition 5.10, more precisely (5.24), shows that $\delta\left(\mathbf{i} \iota \alpha^{*} \mathbf{e}\right)=\delta\left(\mathbf{l} \mathbf{i} \alpha^{*} \mathbf{e}\right)$ in $\mathrm{ENH}_{\mathrm{k}}$. Hence $\delta\left(\mathbf{i} \alpha_{*} \mathbf{i} \mathbf{L} \alpha^{*} \mathbf{e}\right)=\delta\left(\mathbf{i} \alpha_{*} \mathbf{i} \alpha^{*} \mathbf{e}\right)$, i. e. the bent green arrow and the cyan arrow become equal in $\mathrm{ENH}_{\mathrm{k}}$. This implies our claim. 


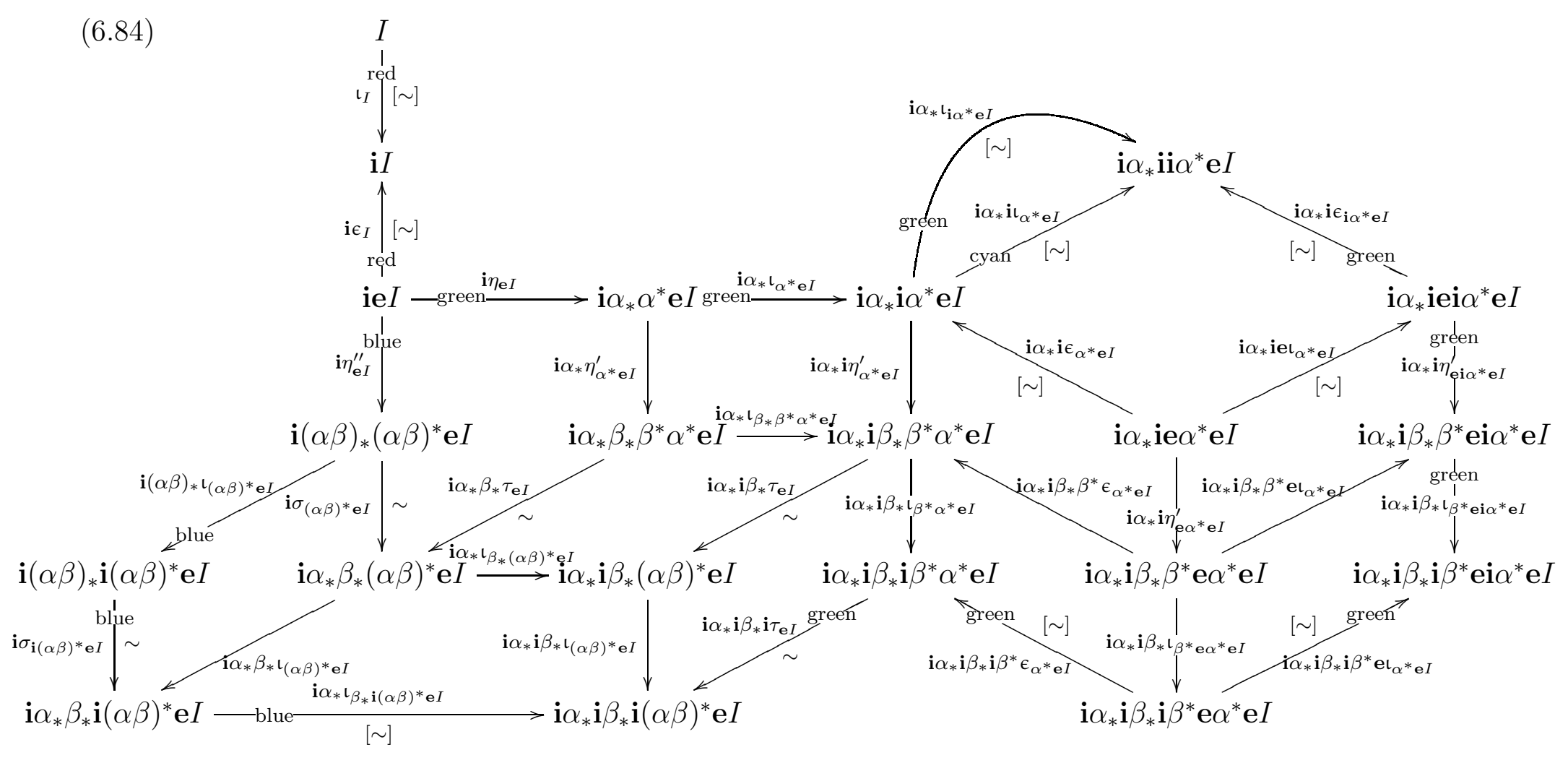


Recall that a (symmetric) monoidal category is a (symmetric) monoidal object in the 2-category of categories. We generalize this definitions in the obvious way to k-linear 2-multicategories.

Lemma 6.29. Let $(\mathcal{X}, \mathcal{O})$ be a $\mathrm{k}$-ringed site, define $l:=(6.70), r:=(6.71)$, $a:=$ (6.72), s $:=(6.73)$, and consider $\underline{\mathcal{O}}$ as a 1-morphism $\underline{\mathcal{O}}: \emptyset \rightarrow \underline{\mathbb{I}}(\mathcal{X})$, cf. Remark 5.8. Then $(\underline{\mathbb{I}}(\mathcal{X}), \underline{\otimes}, \underline{\mathcal{O}}, a, l, r, s)$ is a symmetric monoidal object in $\mathrm{ENH}_{\mathrm{k}}$, i. e. the diagrams (1.4), (1.5), (1.6) in $\mathrm{ENH}_{\mathrm{k}}$ commute.

Proof. Obvious and left to the reader.

Remark 6.30. One may call $(\underline{\mathbb{I}}(\mathcal{X}), \underline{\mathbb{Q}}, \underline{\mathcal{O}}, a, l, r, s)$ a "symmetric monoidal enhancement"; similar terminology in the context of derivators appears in [Gro12, 2.1]). If we apply the functor (5.13), we obtain the symmetric monoidal triangulated k-category $[\underline{I}(\mathcal{X})]$. Lemma 6.22 shows that $(6.3)$ is an equivalence of symmetric monoidal triangulated k-categories.

6.5.3. Some other lifts. Recall that each object $G \in \mathrm{C}(\mathcal{X})$ gives rise to an object $\underline{G}:=\mathrm{i} G$ of $\mathbb{I}(\mathcal{X})$ and to a 1-morphism $\underline{G}$ in $\mathrm{ENH}_{\mathrm{k}}$, cf. (6.20). Similarly, any morphism $g: G \rightarrow G^{\prime}$ in $\mathrm{C}(\mathcal{X})$ gives rise to a morphism

$$
\underline{q}:=\mathrm{i} g: \underline{G} \rightarrow \underline{G}^{\prime}
$$

in $\mathbb{I}(\mathcal{X})$ and to a 2-morphism $\underline{q}: \underline{G} \rightarrow \underline{G^{\prime}}$ in $\mathrm{ENH}_{\mathrm{k}}$.

Lemma 6.31. Let $\alpha:\left(\operatorname{Sh}(\mathcal{Y}), \mathcal{O}_{\mathcal{Y}}\right) \rightarrow\left(\operatorname{Sh}(\mathcal{X}), \mathcal{O}_{\mathcal{X}}\right)$ be a morphism of $\mathrm{k}$-ringed topoi, let $E, F \in \mathrm{C}(\mathcal{X})$, and let $g: G \rightarrow G^{\prime}$ be a morphism in $\mathrm{C}(\mathcal{Y})$. Then the zig-zags

$$
\underline{\mathcal{H} o m}(E, F)=\mathbf{i} \mathcal{H} o m(E, F) \rightarrow \mathbf{i} \mathcal{H} o m(E, \mathbf{i} F) \stackrel{[\sim]}{\longleftarrow} \mathbf{i} \mathcal{H o m}(\mathbf{i} E, \mathbf{i} F)=\underline{\mathcal{H} o m}(\underline{E}, \underline{F}),
$$

$$
\begin{aligned}
& \underline{\mathrm{C}}(E, F)=\underline{\mathrm{C}}(E, F) \rightarrow \underline{\mathrm{C}}(E, \mathbf{i} F) \stackrel{[\sim]}{\longleftarrow} \underline{\mathrm{C}}(\mathbf{i} E, \mathbf{i} F)=\underline{\operatorname{Hom}}(\mathbf{i} E, \mathbf{i} F)=\underline{\operatorname{Hom}}(\underline{E}, \underline{F}), \\
& \underline{G} \stackrel{\underline{q}}{\rightarrow} \underline{G^{\prime}}
\end{aligned}
$$


of obvious 2-morphisms in $\widetilde{\mathrm{ENH}}_{\mathrm{k}}$ define 2-morphisms

$$
\begin{aligned}
\underline{\alpha}^{*} \underline{E} & \rightarrow \underline{\alpha^{*} E} \\
\underline{E} \underline{\otimes} \underline{F} & \rightarrow \underline{E \otimes F} \\
\underline{\alpha_{*} G} & \rightarrow \underline{\alpha} \underline{G}^{G}
\end{aligned}
$$

(2-isomorphism if $E$ is h-flat),

$\underline{\mathcal{H o m}(E, F)} \rightarrow \underline{\mathcal{H} o m}(\underline{E}, \underline{F}) \quad$ (2-isom. if $F$ h-inj., or E h-flat, F weakly h-inj.),

$$
\begin{array}{r}
\underline{\underline{\mathrm{C}}(E, F)} \rightarrow \underline{\operatorname{Hom}}(\underline{E}, \underline{F}) \quad \text { (2-isom. if } F \text { h-inj., or } E \text { h-flat, } F \text { weakly h-inj.), } \\
\underline{G} \stackrel{\underline{g}}{\rightarrow} \underline{G^{\prime}} \\
\text { (2-isom. if } g \text { is a quasi-isomorphism) }
\end{array}
$$

in $\mathrm{ENH}_{\mathrm{k}}$ that are 2-isomorphisms in the indicated cases and that enhance the 2morphisms $\mathbf{L} \alpha^{*}(E) \rightarrow \alpha^{*} E, E \otimes^{\mathbf{L}} F \rightarrow E \otimes F, \alpha_{*} G \rightarrow \mathbf{R} \alpha_{*}(G), \mathcal{H o m}(E, F) \rightarrow$ $\mathbf{R H o m}(E, F), \underline{\mathrm{C}}(E, F) \rightarrow \mathbf{R H o m}(E, F), G \stackrel{g}{\rightarrow} G^{\prime}$. The first five of these 2-morphisms come from the k-natural transformation which is part of the datum of the corresponding derived functor. In particular, (6.92) provides a 2-isomorphism $\underline{\alpha}^{*} \underline{\mathcal{O}}_{\mathcal{X}} \stackrel{\sim}{\rightarrow} \underline{\alpha^{*} \mathcal{O}_{\mathcal{X}}}=$ $\underline{\mathcal{O}}_{\mathcal{Y}}$ in $\mathrm{ENH}_{\mathrm{k}}$ that enhances $\mathbf{L} \alpha^{*} \mathcal{O}_{\mathcal{X}} \stackrel{\sim}{\rightarrow} \mathcal{O}_{\mathcal{Y}}$.

Proof. Left to the reader.

Lemma 6.32. Let $\alpha:\left(\operatorname{Sh}(\mathcal{Y}), \mathcal{O}_{\mathcal{Y}}\right) \rightarrow\left(\operatorname{Sh}(\mathcal{X}), \mathcal{O}_{\mathcal{X}}\right)$ be a morphism of k-ringed topoi, let $e: E \rightarrow E^{\prime}, f: F \rightarrow F^{\prime}$ be morphisms in $\mathrm{C}(\mathcal{X})$, let $g, g^{\prime}: G \rightarrow G^{\prime}$ and $h: G^{\prime} \rightarrow G^{\prime \prime}$ be morphisms in $\mathrm{C}(\mathcal{Y})$, and let $r, r^{\prime} \in \mathrm{k}$. Then the following diagrams in $\mathrm{ENH}_{\mathrm{k}}$ are commutative.
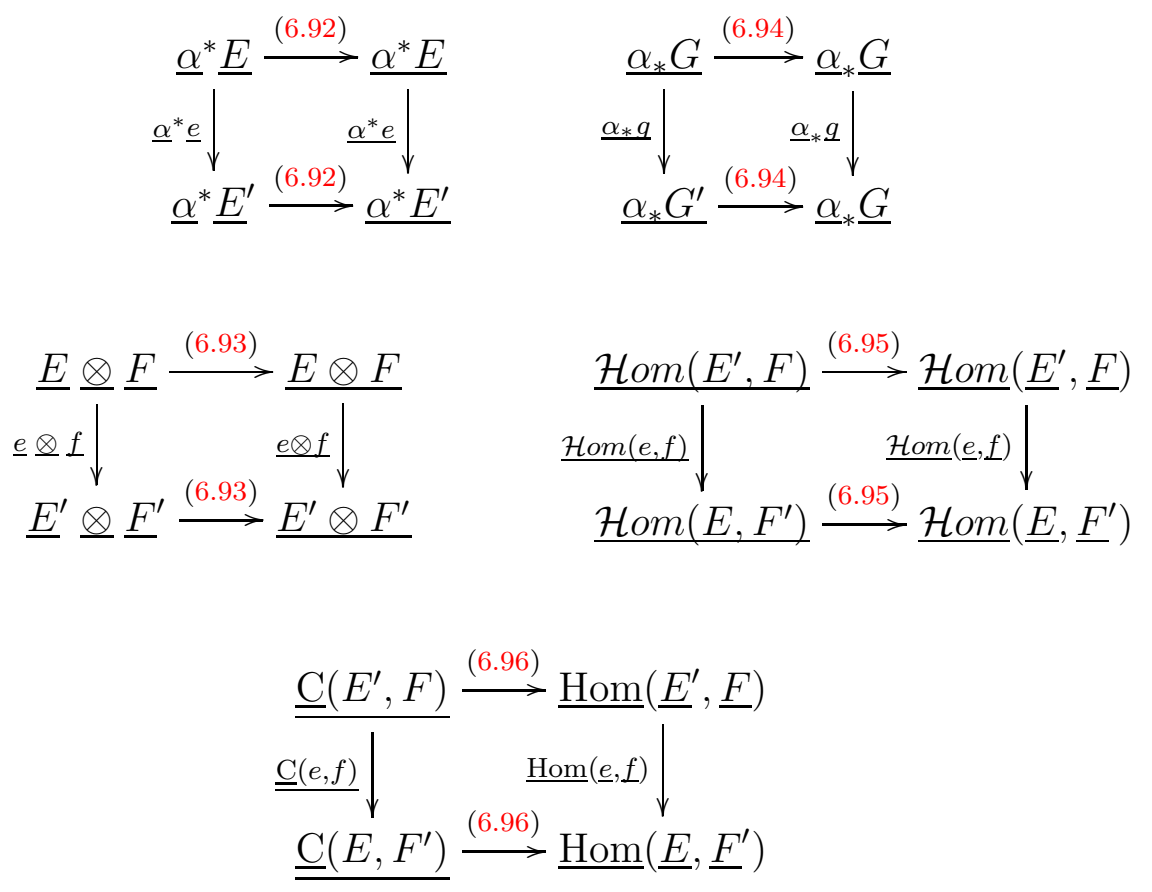


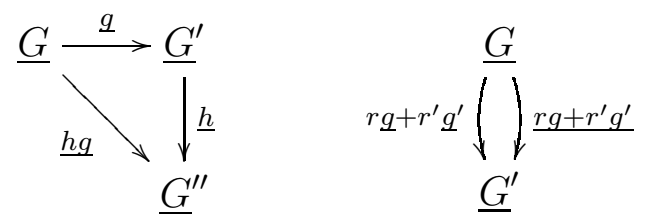

Proof. Left to the reader. The last two diagrams are commutative because the $\mathcal{K}$ functor $\mathbf{i}: \underline{\mathrm{C}}(\mathcal{Y}) \rightarrow \underline{\mathbb{I}}(\mathcal{Y})$ gives rise to a k-functor $\mathrm{C}(\mathcal{Y}) \rightarrow \mathbb{I}(\mathcal{Y}) \rightarrow[\underline{\mathbb{I}}(\mathcal{Y})]$.

6.5.4. Subsequently constructed lifts of 2-(iso)morphisms. We give some examples as to how to produce more lifts from the lifts we have constructed so far.

Lemma 6.33. Let $\alpha:\left(\operatorname{Sh}(\mathcal{Y}), \mathcal{O}_{\mathcal{Y}}\right) \rightarrow\left(\operatorname{Sh}(\mathcal{X}), \mathcal{O}_{\mathcal{X}}\right)$ be a morphism of $\mathrm{k}$-ringed topoi. Then the composition

$$
\begin{aligned}
\underline{\operatorname{Hom}}\left(\underline{\alpha}^{*}-,-\right) \stackrel{(6.75)\left(\underline{\alpha}^{*}, \mathrm{id}\right)}{\sim} & \Gamma \frac{\mathcal{H} \operatorname{Hom}}{\sim}\left(\underline{\alpha}^{*}-,-\right) \frac{(6.64) \underline{\mathcal{H o m}}\left(\underline{\alpha}^{*}-,-\right)}{\sim} \underline{\Gamma} \underline{\alpha}_{*} \underline{\mathcal{H} o m}\left(\underline{\alpha}^{*}-,-\right) \\
\stackrel{\underline{\Gamma}(6.78)}{\sim} & \Gamma \underline{\mathcal{H} o m}\left(-, \underline{\alpha}_{*}-\right) \frac{(6.75)^{-1}\left(\mathrm{id}, \underline{\alpha}_{*}\right)}{\sim} \underline{\operatorname{Hom}}\left(-, \underline{\alpha}_{*}-\right)
\end{aligned}
$$

of 2-isomorphisms in $\mathrm{ENH}_{\mathbf{k}}$ enhances $\mathbf{R} \operatorname{Hom}\left(\mathbf{L} \alpha^{*}-,-\right) \stackrel{\sim}{\rightarrow} \mathbf{R} \operatorname{Hom}\left(-, \mathbf{R} \alpha_{*}-\right)$.

Proof. Use Remark 6.16, Lemmas 6.21, 6.23, and Proposition 6.25.

Remark 6.34. Let us explain how the adjunction isomorphisms $\mathrm{D}_{\mathcal{Y}}\left(\mathbf{L} \alpha^{*} E, F\right) \stackrel{\sim}{\rightarrow}$ $\mathrm{D}_{\mathcal{X}}\left(E, \mathbf{R} \alpha_{*} F\right)$ are encoded in this formalism. Lemma 6.33 implies that the diagram

$$
\begin{aligned}
& \left.[\underline{\operatorname{Hom}}]\left(\left[\underline{\alpha}^{*}\right] \overline{\mathbf{i}]}\right](-), \overline{[\mathbf{i}]}(-)\right) \stackrel{[(6.102)] \overline{\mathbf{i}]}}{\sim}[\underline{\operatorname{Hom}}]\left(\overline{[\mathbf{i}]}(-),\left[\underline{\alpha}_{*}\right] \overline{[\mathbf{i}]}(-)\right) \\
& \omega_{\mathrm{Hom}\left(\alpha^{*}(-),-\right)} \downarrow \sim \quad \omega_{\mathrm{Hom}\left(-, \alpha_{*}(-)\right)} \downarrow \sim \\
& \mathbf{R H o m}\left(\mathbf{L} \alpha^{*}(-),-\right) \longrightarrow \mathbf{R H o m}\left(-, \mathbf{R} \alpha_{*}(-)\right)
\end{aligned}
$$

of 1- and 2-morphisms in TRCAT Th commutes, cf. (6.39) (we use that $\overline{\left[\mathbf{i}_{(\mathrm{pt}, \mathrm{k})}\right]}=\mathrm{id}$ ). If we evaluate this diagram at $E \in \mathrm{D}(\mathcal{X})$ and $F \in \mathrm{D}(\mathcal{Y})$, take $H^{0}:[\mathcal{K}] \rightarrow \operatorname{Mod}(\mathrm{k})$ and use Remark 6.4 we obtain the upper square in the commutative diagram

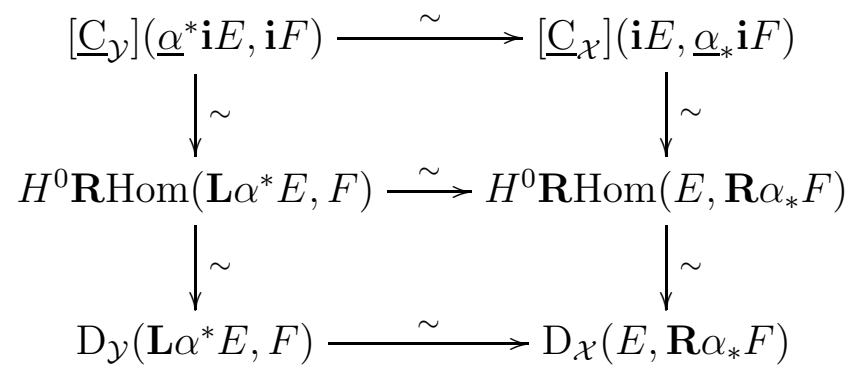

in $\operatorname{Mod}(\mathrm{k})$. The vertical arrows in the lower square are the usual isomorphisms and the lower horizontal isomorphism is the adjunction isomorphism. 
Lemma 6.35. Let $\left(\mathcal{X}, \mathcal{O}_{\mathcal{X}}\right)$ be a $\mathrm{k}$-ringed site. Then the composition

$$
\begin{aligned}
& \underline{\operatorname{Hom}}(-\underline{\otimes}-,-) \stackrel{(6.75)(-\underline{\otimes}-,-)}{\sim} \underline{\Gamma} \underline{\mathcal{H} o m}(-\underline{\otimes}-,-) \\
& \stackrel{\Gamma(6.80)}{\sim} \underline{\Gamma} \underline{\mathcal{H} o m}(-, \underline{\mathcal{H} o m}(-,-)) \frac{(6.75)^{-1}(-, \mathcal{H o m}(-,-))}{\sim} \underline{\operatorname{Hom}}(-, \underline{\mathcal{H o m}}(-,-))
\end{aligned}
$$

of 2-isomorphisms in $\mathrm{ENH}_{\mathbf{k}}$ enhances $\mathbf{R H o m}\left(-\otimes^{\mathbf{L}}-,-\right) \stackrel{\sim}{\rightarrow} \mathbf{R H o m}(-, \mathbf{R H o m}(-,-))$.

The adjunction isomorphisms $\mathrm{D}_{\mathcal{X}}\left(E \otimes{ }^{\mathbf{L}} F, G\right) \cong \mathrm{D}_{\mathcal{Y}}(E, \mathbf{R} \mathcal{H} o m(F, G))$ can be obtained as in Remark 6.34.

Proof. Use Remark 6.16, Lemma 6.23 and Proposition 6.26.

Lemma 6.36. Let $\alpha:\left(\operatorname{Sh}(\mathcal{Y}), \mathcal{O}_{\mathcal{Y}}\right) \rightarrow\left(\operatorname{Sh}(\mathcal{X}), \mathcal{O}_{\mathcal{X}}\right)$ be a morphism of $\mathrm{k}$-ringed topoi. Then the composition

$\underline{\alpha}_{*} \underline{\mathcal{H} o m}(-,-) \stackrel{\underline{\alpha}_{*}}{\stackrel{\mathcal{H} o m}{((6.42),-)} \longrightarrow} \underline{\alpha}_{*} \underline{\mathcal{H} o m}\left(\underline{\alpha}^{*} \underline{\alpha}_{*}(-),-\right) \stackrel{(6.78)\left(\underline{\alpha}_{*}(-),-\right)}{\sim} \underline{\mathcal{H} o m}\left(\underline{\alpha}_{*}(-), \underline{\alpha}_{*}(-)\right)$

in $\mathrm{ENH}_{\mathrm{k}}$ enhances $\mathbf{R} \alpha_{*} \mathbf{R} \mathcal{H}$ om $(-,-) \rightarrow \mathbf{R} \mathcal{H o m}\left(\mathbf{R} \alpha_{*}(-), \mathbf{R} \alpha_{*}(-)\right)$, cf. [KS94, (2.6.24)].

Proof. Obvious and left to the reader.

Remark 6.37. Some of the 2-(iso)morphisms in $\mathrm{ENH}_{\mathrm{k}}$ admit alternative constructions. For example, the compositions in (6.102), (6.105), (6.106) can be constructed directly from zig-zags, cf. Propositions 6.25, 6.26.

\section{TWO OPERATIONS}

We lift the remaining part of Grothendieck-Verdier-Spaltenstein's six functor for-

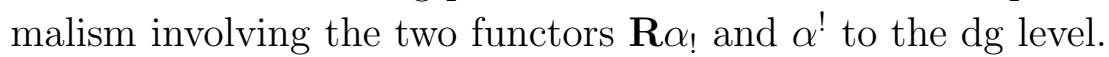

All ringed spaces and algebras in this section are assumed to be $\mathcal{U}$-small; all algebras are assumed to be commutative.

7.1. Proper direct image. We use results on the proper direct image functor and on separated and locally proper morphisms of topological spaces from [SS16]; all results there extend in a straightforward manner from sheaves of abelian groups to sheaves of modules over some fixed ground ring (for the modification of [SS16, Thm. 6.3] cf. [KS94, Prop. 2.6.6]), and we use these extended results tacitly; instead of the notation $\alpha_{(!)}, \alpha_{!}$, etc. there we use the traditional notation $\alpha_{!}, \mathbf{R} \alpha_{\text {! }}$ etc. here.

Let $\mathrm{R}$ be a ring and $A$ a (commutative) R-algebra. If $X$ is a topological space, the constant sheaf $A_{X}$ of R-algebras with stalk $A$ turns $X$ into an R-ringed space $X_{A}:=\left(X, A_{X}\right)$. Any morphism $\alpha: Y \rightarrow X$ of topological spaces gives rise to a morphism $\alpha: Y_{A} \rightarrow X_{A}$ of R-ringed spaces whose comorphism is adjoint to the identity morphism $\alpha^{-1} A_{X} \stackrel{\text { id }}{\rightarrow} A_{Y}$. Then $\alpha^{-1}=\alpha^{*}: \operatorname{Mod}\left(X_{A}\right) \rightarrow \operatorname{Mod}\left(Y_{A}\right)$ is an exact functor 
and $\alpha^{-1}=\alpha^{*}: \underline{\mathrm{C}}\left(X_{A}\right) \rightarrow \underline{\mathrm{C}}\left(Y_{A}\right)$ and $\mathbf{L} \alpha^{-1}=\mathbf{L} \alpha^{*}: \mathrm{D}\left(X_{A}\right) \rightarrow \mathrm{D}\left(Y_{A}\right)$. To emphasize that we work with sheaves of $A$-modules we usually prefer the notation $\alpha^{-1}$ and $\mathbf{L} \alpha^{-1}$.

Let $\alpha_{!}: \operatorname{Mod}\left(Y_{A}\right) \rightarrow \operatorname{Mod}\left(X_{A}\right)$ denote the proper direct image functor. It induces the $\mathcal{R}$-functor $\alpha_{!}: \underline{\mathrm{C}}\left(Y_{A}\right) \rightarrow \underline{\mathrm{C}}\left(X_{A}\right)$ and the derived functor $\mathbf{R} \alpha_{!}: \mathrm{D}\left(Y_{A}\right) \rightarrow \mathrm{D}\left(X_{A}\right)$.

If $\alpha$ is a separated and locally proper morphism of topological spaces such that $\alpha_{!}: \operatorname{Mod}\left(Y_{A}\right) \rightarrow \operatorname{Mod}\left(X_{A}\right)$ has finite cohomological dimension, then $\mathbf{R} \alpha_{\text {! }}$ admits a right adjoint functor $\alpha^{!}: \mathrm{D}\left(X_{A}\right) \rightarrow \mathrm{D}\left(Y_{A}\right)$, by [SS16, Thm. 8.3.(a)]. We fix an adjunction $\left(\mathbf{R} \alpha_{!}, \alpha^{!}\right)$.

7.2. The multicategory of formulas for the six operations. Recall the category $\mathrm{fml}_{\mathrm{R}}^{\prime}$ of formulas from 5.4. For each R-algebra $A$ and each topological space $X$ we have the object $X_{A}:=X_{A}$ of this category.

Let $\mathrm{fml}_{\mathrm{R}}$ be the free multicategory having the same objects as $\mathrm{fml}_{\mathrm{R}}^{\prime}$ and whose generating morphisms consist of the generating morphisms of $\mathrm{fml}_{\mathrm{R}}^{\prime}$ and the following morphisms:

(a) for each R-algebra $A$ and each morphism $\alpha: Y \rightarrow X$ of topological spaces there are morphisms $\alpha^{-1}: X_{A} \rightarrow Y_{A}$ and $\alpha_{!}: Y_{\ldots A} \rightarrow X_{A}$ and their opposites $\left(\alpha^{-1}\right)^{\text {op }}: X_{A}^{\mathrm{op}} \rightarrow Y_{A}^{\mathrm{op}}$ and $\alpha_{!}^{\mathrm{op}}: Y_{A}^{\mathrm{op}} \rightarrow X_{A}^{\mathrm{op}}$

(b) for each R-algebra $A$ and each separated, locally proper morphism $\alpha: Y \rightarrow X$ with $\alpha_{!}: \operatorname{Mod}\left(Y_{A}\right) \rightarrow \operatorname{Mod}\left(X_{A}\right)$ of finite cohomological dimension there are a morphism $\alpha^{!}: X_{A} \rightarrow Y_{A}$ and its opposite $\left(\alpha^{!}\right)^{\text {op }}: X_{A}^{\text {op }} \rightarrow Y_{A}^{\text {op }}$.

Obviously, fml $\mathrm{R}_{\mathrm{R}}$ contains $\mathrm{fml}_{\mathrm{R}}^{\prime}$ as a full subcategory, and the involution $(-)^{\mathrm{op}}$ on $\mathrm{fml}_{\mathrm{R}}^{\prime}$ extends to $\mathrm{fml}_{\mathrm{R}}$. The interpretation functor (5.38) of formulas in triangulated categories extends uniquely to a functor

$$
\mathrm{D}: \mathrm{fml}_{\mathrm{R}} \rightarrow \text { trcat }_{\mathrm{R}}
$$

of multicategories by $\alpha^{-1} \mapsto \mathbf{L} \alpha^{-1}, \alpha_{!} \mapsto \mathbf{R} \alpha_{!}, \alpha^{!} \mapsto \alpha^{!}$.

7.3. More fixed data. We keep the conventions from 6.1. Additionally, for each k-algebra $A$ and each separated, locally proper morphism $\alpha: Y \rightarrow X$ of topological spaces with $\alpha_{!}: \operatorname{Mod}\left(Y_{A}\right) \rightarrow \operatorname{Mod}\left(X_{A}\right)$ of finite cohomological dimension, we fix a bounded complex $\mathcal{L}=\mathcal{L}_{\alpha} \in \mathrm{C}\left(Y_{A}\right)$ with flat and $\alpha$-c-soft components and a quasiisomorphism

$$
A_{Y} \rightarrow \mathcal{L}=\mathcal{L}_{\alpha}
$$

in $\mathrm{C}\left(Y_{A}\right)$; this is possible by the proof of [SS16, Thm. 7.7].

Proposition 7.1. Let $A$ be a k-algebra and $\alpha: Y \rightarrow X$ a separated, locally proper morphism of topological spaces with $\alpha_{!}: \operatorname{Mod}\left(Y_{A}\right) \rightarrow \operatorname{Mod}\left(X_{A}\right)$ of finite cohomological dimension. Let $\mathcal{M} \in \mathrm{C}\left(Y_{A}\right)$ be a bounded complex of flat $A_{Y}$-modules such that one of the following two conditions is satisfied.

(a) All components of $\mathcal{M}$ are $\alpha$-c-soft. 
(b) For all components $\mathcal{M}^{p}$ of $\mathcal{M}$ the functor $\left(-\otimes \mathcal{M}^{p}\right): \operatorname{Mod}\left(Y_{A}\right) \rightarrow \operatorname{Mod}\left(Y_{A}\right)$ lands in the subcategory of $\alpha_{!}$-acyclic objects.

Then the $\mathcal{K}$-functor

$$
\alpha_{!}^{\mathcal{M}}:=\alpha_{!}(\mathcal{M} \otimes-): \underline{\mathrm{C}}\left(Y_{A}\right) \rightarrow \underline{\mathrm{C}}\left(X_{A}\right)
$$

admits a right adjoint $\mathcal{K}$-functor

$$
\alpha_{\mathcal{M}}^{!}: \underline{\mathrm{C}}\left(X_{A}\right) \rightarrow \underline{\mathrm{C}}\left(Y_{A}\right) .
$$

Moreover, $\alpha_{!}^{\mathcal{M}}$ preserves acyclic objects and $\alpha_{\mathcal{M}}^{!}$preserves h-injective objects, componentwise injective objects and, in particular, I-fibrant objects.

Proof. If condition (a) holds, this follows from [SS16, Prop. 7.5 and proofs of Thms 7.7 and 8.3.(a)]. By inspection of the proof of [SS16, Prop. 7.5] we see that condition (b) is also sufficient for our conclusion.

Given any datum as in Proposition 7.1 we fix an adjunction $\left(\alpha_{!}^{\mathcal{M}}, \alpha_{\mathcal{M}}^{!}\right)$in $\operatorname{DGCAT}_{\mathrm{k}}$.

Remark 7.2. Subsequent constructions will depend on the fixed componentwise flat and $\alpha$-c-soft resolution (7.2) of $A_{Y}$. Nevertheless the constructions obtained from different choices are easy to compare, starting from the following observation: if $A_{Y} \rightarrow \mathcal{L}^{\prime}$ is another componentwise flat and $\alpha$-c-soft resolution, its tensor product with $A_{Y} \rightarrow \mathcal{L}=\mathcal{L}_{\alpha}$ provides a resolution $A_{Y} \rightarrow \mathcal{L} \otimes \mathcal{L}^{\prime}$ of the same type which allows the comparison of $\alpha_{!}^{\mathcal{L}}$ and $\alpha_{!}^{\mathcal{L}^{\prime}}$ via $\alpha_{!}^{\mathcal{L} \otimes \mathcal{L}^{\prime}}$, and then also of $\alpha_{\mathcal{L}}^{!}$and $\alpha_{\mathcal{L}^{\prime}}^{!}$, by Remark 7.24 below.

7.4. Lifts of derived functors. Let $A$ be a k-algebra and $\alpha: Y \rightarrow X$ a morphism of topological spaces. Define $\mathcal{K}$-functors

$$
\begin{array}{r}
\underline{\alpha}_{!}:=\mathbf{i} \alpha_{!}: \underline{\mathbb{I}}\left(Y_{A}\right) \stackrel{\alpha_{!}}{\rightarrow} \underline{\mathrm{C}}\left(X_{A}\right) \stackrel{\mathbf{i}}{\rightarrow} \underline{\mathbb{I}}\left(X_{A}\right), \\
\underline{\alpha}^{-1}:=\mathbf{i} \alpha^{-1}: \underline{\mathbb{I}}\left(X_{A}\right) \stackrel{\alpha^{-1}}{\longrightarrow} \underline{\mathrm{C}}\left(Y_{A}\right) \stackrel{\mathbf{i}}{\rightarrow} \underline{\mathbb{I}}\left(Y_{A}\right) .
\end{array}
$$

Similarly to (6.10), (6.9), there are canonical 2-isomorphisms

$$
\begin{gathered}
\omega_{\alpha_{!}}:\left[\underline{\alpha}_{!}\right] \overline{[\mathbf{i}]} \stackrel{\sim}{\rightarrow} \overline{[\mathbf{i}]} \mathbf{R} \alpha_{!}, \\
\omega_{\alpha^{-1}}:\left[\underline{\alpha}^{-1}\right] \overline{\mathbf{i}]} \stackrel{\sim}{\rightarrow} \overline{[\mathbf{i}]} \mathbf{L} \alpha^{-1} .
\end{gathered}
$$

If $\alpha$ is separated and locally proper with $\alpha_{!}: \operatorname{Mod}\left(Y_{A}\right) \rightarrow \operatorname{Mod}\left(X_{A}\right)$ of finite cohomological dimension, Proposition 7.1 allows to define the $\mathcal{K}$-functor

$$
\underline{\alpha}^{!}:=\alpha_{\mathcal{L}}^{!}=\alpha_{\mathcal{L}_{\alpha}}^{!}: \underline{\mathbb{I}}\left(X_{A}\right) \rightarrow \underline{\mathbb{I}}\left(Y_{A}\right) .
$$

It follows from the proof of [SS16, Thm. 8.3.(a)] that the composition

$$
\mathrm{D}\left(X_{A}\right) \stackrel{\overline{\mathbf{i}]}}{\longrightarrow}\left[\underline{\mathbb{I}}\left(X_{A}\right)\right] \stackrel{\left[\underline{\alpha}^{\prime}\right]}{\longrightarrow}\left[\underline{\mathbb{I}}\left(Y_{A}\right)\right] \rightarrow\left[\underline{\mathrm{C}}\left(Y_{A}\right)\right] \stackrel{q_{Y_{A}}}{\longrightarrow} \mathrm{D}\left(Y_{A}\right)
$$


is right adjoint to $\mathbf{R} \alpha_{\text {! }}$ with explicitly given unit and counit morphisms obtained from $\left(\alpha_{!}^{\mathcal{L}}, \alpha_{\mathcal{L}}^{!}\right)$, and therefore canonically isomorphic to $\alpha^{!}$. We obtain a canonical 2-isomorphism

$$
\omega_{\alpha^{!}}:[\underline{\alpha} !] \overline{\mathbf{i}]} \stackrel{\sim}{\rightarrow} \overline{[\mathbf{i}]} \alpha^{!} .
$$

The results of 6.4 generalize in the obvious way. The interpretation functor (6.32) of formulas in enhancements extends uniquely to a functor

$$
\underline{I}: \mathrm{fml}_{\mathrm{k}} \rightarrow \mathrm{enh}_{\mathrm{k}}
$$

of multicategories by $\alpha^{-1} \mapsto \underline{\alpha}^{-1}, \alpha_{!} \mapsto \underline{\alpha}_{!}, \alpha^{!} \mapsto \underline{\alpha}^{!}$. The pseudo-natural transformation (6.33) accordingly extends by mapping $\alpha_{!}, \alpha^{-1}, \alpha^{!}$to $\omega_{\alpha_{!}}, \omega_{\alpha^{-1}}, \omega_{\alpha^{!}}$, respectively. Definition 6.15 and all results using this definition extend to and will be used for arbitrary morphisms $\underline{v}$ and $\underline{w}$ in $\mathrm{fml}_{\mathrm{k}}$.

\subsection{Lifts of relations.}

7.5.1. Lifts of 2-(iso)morphisms.

Lemma 7.3. Let $A$ be a k-algebra and $\alpha: Y \rightarrow X$ a morphism of topological spaces. The obvious objectwise homotopy equivalence $\underline{\alpha}^{*}=\mathbf{i} \alpha^{*} \mathbf{e}=\mathbf{i} \alpha^{-1} \mathbf{e} \stackrel{[\sim]}{\longrightarrow} \mathbf{i} \alpha^{-1}=\underline{\alpha}^{-1}$ in $\widetilde{\mathrm{ENH}}_{\mathrm{k}}$ defines an isomorphism

$$
\underline{\alpha}^{*} \stackrel{\sim}{\rightarrow} \underline{\alpha}^{-1}
$$

in $\mathrm{ENH}_{\mathrm{k}}$ which enhances the identity $\mathbf{L} \alpha^{*}=\mathbf{L} \alpha^{-1}$.

Proof. Obvious.

We deduce the following consequence.

Lemma 7.4. Let $A$ be a k-algebra and $\alpha: Y \rightarrow X$ a morphism of topological spaces. The compositions

$$
\begin{array}{r}
\underline{\alpha}^{-1} \underline{\alpha}_{*} \stackrel{(7.13)^{-1} \underline{\alpha}_{*}}{\sim} \underline{\alpha}^{*} \underline{\alpha}_{*} \stackrel{(6.42)}{\longrightarrow} \text { id, } \\
\quad \text { id } \stackrel{(6.44)}{\longrightarrow} \underline{\alpha}_{*} \underline{\alpha}^{*} \stackrel{\underline{\alpha}_{*}^{(7.13)}}{\sim} \underline{\alpha}_{*} \underline{\alpha}^{-1}
\end{array}
$$

of 2-morphisms in $\mathrm{ENH}_{\mathrm{k}}$ enhance counit $\mathbf{L} \alpha^{-1} \mathbf{R} \alpha_{*} \rightarrow$ id and unit id $\rightarrow \mathbf{R} \alpha_{*} \mathbf{L} \alpha^{-1}$ of the adjunction $\left(\mathbf{L} \alpha^{-1}, \mathbf{R} \alpha_{*}\right)$. Moreover, the two 1-morphisms $\underline{\alpha}^{-1}$ and $\underline{\alpha}_{*}$ and the two 2-morphisms (7.15) and (7.14) form an adjunction in $\mathrm{ENH}_{\mathrm{k}}$.

Proof. The first statement follows from Proposition 6.17 and Lemma 7.3. The second statement follows from Proposition 6.27 
Lemma 7.5. Let $A$ be a k-algebra and $\alpha: Y \rightarrow X$ a morphism of topological spaces. Then the obvious 2-morphism $\alpha_{!} \rightarrow \alpha_{*}$ between 1-morphisms $\underline{\mathrm{C}}\left(Y_{A}\right) \rightarrow \underline{\mathrm{C}}\left(X_{A}\right)$ in DGCAT $_{\mathrm{k}}$ induces a 2-morphism $\underline{\alpha}_{!}=\mathbf{i} \alpha_{!} \rightarrow \mathbf{i} \alpha_{*}=\underline{\alpha}_{*}$ in $\widetilde{\mathrm{ENH}}_{\mathrm{k}}$ and then a 2-morphism

$$
\underline{\alpha}_{!} \rightarrow \underline{\alpha}_{*}
$$

in $\mathrm{ENH}_{\mathrm{k}}$ that enhances $\mathbf{R} \alpha_{!} \rightarrow \mathbf{R} \alpha_{*}$. If $\alpha$ is proper, then (7.16) is a 2-isomorphism.

Proof. Clearly, $\underline{\alpha}_{!} \rightarrow \underline{\alpha}_{*}$ enhances $\mathbf{R} \alpha_{!} \rightarrow \mathbf{R} \alpha_{*}$. If $\alpha$ is proper then $\alpha_{!}=\alpha_{*}$ and hence $\underline{\alpha}_{!} \rightarrow \underline{\alpha}_{*}$ is a 2-isomorphism.

We need some preparations for Proposition 7.9.

Remark 7.6. For $\alpha=\operatorname{id}_{X}$ the morphism id $\stackrel{\sim}{\rightarrow} A_{X} \otimes(-) \stackrel{(7.2) \otimes(-)}{\longrightarrow} \mathcal{L} \otimes(-)=\mathrm{id}_{!}^{\mathcal{L}}$ between left adjoints induces, by Remark 7.24 below, a morphism

$$
\mathrm{id}_{\mathcal{L}}^{!} \rightarrow \mathcal{H o m}\left(A_{X},-\right) \stackrel{\sim}{\rightarrow} \mathrm{id}
$$

between the corresponding right adjoints. We have $\mathrm{id}_{\mathcal{L}}^{!} \cong \mathcal{H} \operatorname{om}(\mathcal{L},-)$. In particular, the evaluation of (7.17) at an h-injective object is a quasi-isomorphism, and even a homotopy equivalence because $\mathrm{id}_{\mathcal{L}}^{!}$preserves h-injectives. Of course, we could also assume that $\mathcal{L}_{\mathrm{id}_{X}}=A_{X}$.

Lemma 7.7. Let $A$ be a k-algebra and let $Z \stackrel{\beta}{\rightarrow} Y \stackrel{\alpha}{\rightarrow} X$ be separated, locally proper morphisms of topological spaces with $\alpha_{!}: \operatorname{Mod}\left(Y_{A}\right) \rightarrow \operatorname{Mod}\left(X_{A}\right)$ and $\beta_{!}: \operatorname{Mod}\left(Z_{A}\right) \rightarrow$ $\operatorname{Mod}\left(Y_{A}\right)$ of finite cohomological dimension. Assume that $\mathcal{A} \in \operatorname{Mod}\left(Y_{A}\right)$ is flat and $\alpha$-c-soft and that $\mathcal{B} \in \operatorname{Mod}\left(Z_{A}\right)$ is flat and $\beta$-c-soft. Then $\beta^{-1}(\mathcal{A}) \otimes \mathcal{B} \otimes \mathcal{T}$ is $(\alpha \beta)_{\text {!- }}$ acyclic, for any $\mathcal{T} \in \operatorname{Mod}\left(Z_{A}\right)$.

Proof. Let

$$
0 \rightarrow \beta^{-1}(\mathcal{A}) \otimes \mathcal{B} \otimes \mathcal{T} \rightarrow I^{0} \rightarrow I^{1} \rightarrow \ldots
$$

be an injective resolution. By [SS16, Lemmas 5.5, 7.4], $\beta^{-1}(\mathcal{A}) \otimes \mathcal{B} \otimes \mathcal{T}$ is $\beta$-c-soft and hence $\beta_{!}$-acyclic. Hence

$$
0 \rightarrow \beta_{!}\left(\beta^{-1}(\mathcal{A}) \otimes \mathcal{B} \otimes \mathcal{T}\right) \rightarrow \beta_{!}\left(I^{0}\right) \rightarrow \beta_{!}\left(I^{1}\right) \rightarrow
$$

is an exact sequence. All objects $\beta_{!}\left(I^{n}\right)$ are $\alpha$-c-soft, by [SS16, 5.3, Lemma 5.7], and hence $\alpha_{!}$-acyclic. We also have an isomorphism

$$
\beta_{!}\left(\beta^{-1}(\mathcal{A}) \otimes \mathcal{B} \otimes \mathcal{T}\right) \stackrel{\sim}{\leftarrow} \otimes \beta_{!}(\mathcal{B} \otimes \mathcal{T})
$$

by [SS16, Thm. 6.2], and the object on the right is $\alpha$-c-soft and hence $\alpha_{!}$-acyclic by [SS16, Lemmas 5.5, 7.4]. Hence (7.19) stays exact when we apply $\alpha_{!}$. Hence, using $\alpha_{!} \beta_{!}=(\alpha \beta)_{!}$, see [SS16, Thm. 3.6], (7.18) stays exact when applying $(\alpha \beta)_{!}$. This implies the lemma. 
Remark 7.8. Let $A$ be a k-algebra and let $Z \stackrel{\beta}{\rightarrow} Y \stackrel{\alpha}{\rightarrow} X$ be separated, locally proper morphisms of topological spaces with $\alpha_{!}: \operatorname{Mod}\left(Y_{A}\right) \rightarrow \operatorname{Mod}\left(X_{A}\right)$ and $\beta_{!}: \operatorname{Mod}\left(Z_{A}\right) \rightarrow$ $\operatorname{Mod}\left(Y_{A}\right)$ of finite cohomological dimension. Then $\alpha \beta$ is also separated and locally proper with $(\alpha \beta) ! \operatorname{Mod}\left(Z_{A}\right) \rightarrow \operatorname{Mod}\left(X_{A}\right)$ of finite cohomological dimension, by [SS16, Thm. 8.3.(b)].

Consider the quasi-isomorphisms $A_{Z}=\beta^{-1} A_{Y} \rightarrow \beta^{-1}\left(\mathcal{L}_{\alpha}\right), A_{Z} \rightarrow \mathcal{L}_{\beta}$ and $A_{Z} \rightarrow$ $\mathcal{L}_{\alpha \beta}$ between bounded complexes with flat components. Suitable tensor products of these quasi-isomorphisms yield quasi-isomorphisms

$$
\mathcal{L}_{\alpha \beta} \rightarrow \mathcal{M}:=\mathcal{L}_{\alpha \beta} \otimes \beta^{-1}\left(\mathcal{L}_{\alpha}\right) \otimes \mathcal{L}_{\beta} \leftarrow \mathcal{N}:=\beta^{-1}\left(\mathcal{L}_{\alpha}\right) \otimes \mathcal{L}_{\beta}
$$

between bounded complexes with flat components. We can apply Proposition 7.1 to the map $\alpha \beta$ and the three complexes $\mathcal{L}_{\alpha \beta}, \mathcal{M}$ and $\mathcal{N}$ : this is obvious for $\mathcal{L}_{\alpha \beta}$ and follows from Lemma 7.7 for $\mathcal{M}$ and $\mathcal{N}$. Hence the adjunctions $\left((\alpha \beta)_{!}^{\mathcal{L}_{\alpha \beta}},(\alpha \beta)_{\mathcal{L}_{\alpha \beta}}\right)$, $\left((\alpha \beta) !^{\mathcal{M}},(\alpha \beta)_{\mathcal{M}}\right)$ and $\left.\left((\alpha \beta)_{!}^{\mathcal{N}},(\alpha \beta)\right)_{\mathcal{N}}\right)$ are available.

If $T \in \mathrm{C}\left(Z_{A}\right)$ is arbitrary, applying (-) $\otimes T$ to (7.21) yields quasi-isomorphisms between componentwise $(\alpha \beta)$ !-acyclic complexes, by [SS16, Lemmas 5.5, 7.4] and Lemma 7.7. Hence $(\alpha \beta)$ ! maps these quasi-isomorphisms to quasi-isomorphisms, by $\left[\mathrm{SS} 16\right.$, Lemma 12.4.(b)] using the fact that $(\alpha \beta) !: \operatorname{Mod}\left(Z_{A}\right) \rightarrow \operatorname{Mod}\left(X_{A}\right)$ has finite cohomological dimension. This shows that the evaluation of the zig-zag

$$
(\alpha \beta) !^{\mathcal{L}_{\alpha \beta}} \rightarrow(\alpha \beta) !^{\mathcal{M}} \leftarrow(\alpha \beta)_{!}^{\mathcal{N}}
$$

of 2-morphisms in DGCAT $_{\mathrm{k}}$ at any $T \in \underline{\mathrm{C}}\left(Z_{A}\right)$ is a zig-zag of quasi-isomorphisms. Remark 7.24 below therefore yields a zig-zag

$$
(\alpha \beta)_{\mathcal{L}_{\alpha \beta}} \leftarrow(\alpha \beta)_{\mathcal{M}}^{!} \rightarrow(\alpha \beta)_{\mathcal{N}}^{!}
$$

of 2-morphisms in DGCAT $_{k}$. We claim that the evaluation of this zig-zag at an hinjective object $I \in \underline{\mathrm{C}}\left(X_{A}\right)$ consists of homotopy equivalences (between h-injectives).

Let $T \in \underline{\mathrm{C}}\left(Z_{A}\right)$ and consider the commutative diagram

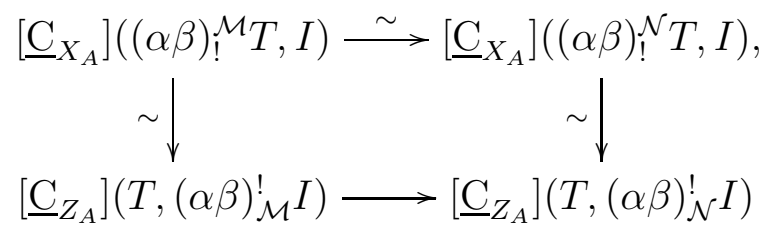

cf. (7.82). Its upper horizontal arrow is an isomorphism because $I$ is h-injective and $(\alpha \beta) !_{!}^{\mathcal{N}} T \rightarrow(\alpha \beta) !^{\mathcal{M}} T$ is a quasi-isomorphism as observed above. The vertical isomorphisms come from the adjunctions. Since $T$ was arbitrary, the Yoneda lemma shows that $(\alpha \beta)_{\mathcal{M}} I \rightarrow(\alpha \beta)_{\mathcal{N}^{\prime}} I$ is an isomorphism in $\left[\underline{\mathrm{C}}\left(Z_{A}\right)\right]$ and hence a homotopy equivalence in $\mathrm{C}\left(Z_{A}\right)$. The same argument with $\mathcal{N}$ replaced by $\mathcal{L}_{\alpha \beta}$ shows the second half of the claim. 
Since $\mathcal{L}_{\alpha}$ has flat components, [SS16, Thms. 3.6, 6.2] provide a 2-isomorphism

$$
\alpha_{!}^{\mathcal{L}_{\alpha}} \beta_{!}^{\mathcal{L}_{\beta}}=\alpha_{!}\left(\mathcal{L}_{\alpha} \otimes \beta_{!}\left(\mathcal{L}_{\beta} \otimes(-)\right)\right) \stackrel{\sim}{\rightarrow} \alpha_{!} \beta_{!}\left(\beta^{-1}\left(\mathcal{L}_{\alpha}\right) \otimes \mathcal{L}_{\beta} \otimes(-)\right)=(\alpha \beta) !
$$

in DGCAT $_{k}$ which corresponds by Remark 7.24 below to a 2-isomorphism

$$
(\alpha \beta)_{\beta^{-1}\left(\mathcal{L}_{\alpha}\right) \otimes \mathcal{L}_{\beta}}^{\stackrel{\sim}{\rightarrow} \beta_{\mathcal{L}_{\beta}}^{!}} \alpha_{\mathcal{L}_{\alpha}}^{!}
$$

Proposition 7.9. Let $A$ be a k-algebra and let $Z \stackrel{\beta}{\rightarrow} Y \stackrel{\alpha}{\rightarrow} X$ be separated, locally proper morphisms of topological spaces with $\alpha_{!}: \operatorname{Mod}\left(Y_{A}\right) \rightarrow \operatorname{Mod}\left(X_{A}\right)$ and $\beta_{!}: \operatorname{Mod}\left(Z_{A}\right) \rightarrow \operatorname{Mod}\left(Y_{A}\right)$ of finite cohomological dimension. Then the zig-zags

$$
\begin{aligned}
& \underline{\mathrm{id}}_{!}=\mathbf{i} \stackrel{[\sim]}{\longleftarrow} \mathrm{id}, \\
& \underline{(\alpha \beta)_{!}}=\mathbf{i}(\alpha \beta)_{!}=\mathbf{i} \alpha_{!} \beta_{!} \stackrel{[\sim]}{\longrightarrow} \mathbf{i} \alpha_{!} \mathbf{i} \beta_{!}=\underline{\alpha}_{!} \underline{\beta}_{!}, \\
& \underline{\mathrm{id}}^{!}=\mathrm{id}_{\mathcal{L}_{\mathrm{id}}} \underset{(7.17)}{\stackrel{[\sim]}{\longrightarrow}} \mathrm{id},
\end{aligned}
$$

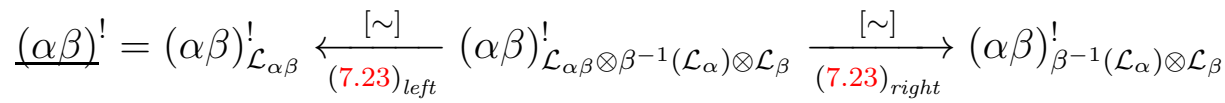

$$
\begin{aligned}
& \underset{(7.26)}{\stackrel{\sim}{\longrightarrow}} \beta_{\mathcal{L}_{\beta}}^{!} \alpha_{\mathcal{L}_{\alpha}}^{!}=\underline{\beta} \underline{\alpha}^{!}
\end{aligned}
$$

of objectwise homotopy equivalences in $\widetilde{\mathrm{ENH}}_{\mathrm{k}}$ define 2-isomorphisms

$$
\begin{aligned}
\underline{\mathrm{id}}_{!} & \sim \mathrm{\sim} \mathrm{id}, \\
(\alpha \beta)_{!} & \stackrel{\sim}{\sim} \underline{\alpha}_{!} \underline{\beta}_{!}, \\
\underline{\mathrm{id}} ! & \stackrel{\sim}{\rightarrow} \mathrm{id}, \\
\underline{(\alpha \beta)^{!}} & \stackrel{\sim}{\rightarrow} \underline{\beta}^{!} \underline{\alpha}^{!}
\end{aligned}
$$

in $\mathrm{ENH}_{\mathrm{k}}$ that enhance the isomorphisms $\mathbf{R i d} ! \cong \mathrm{id}, \mathbf{R}(\alpha \beta)_{!} \cong \mathbf{R} \alpha_{!} \mathbf{R} \beta_{!}$, id! $\cong \mathrm{id}$, $(\alpha \beta)^{!} \cong \beta^{!} \alpha^{!}$from [SS16, Thm. 8.3.(b)]

Proof. The claim for $\underline{\mathrm{id}}_{\text {! }}$ is obvious, and that for $(\alpha \beta)$ ! follows from the proof of [SS16, Thm. 8.3.(b)]. The claims for $\underline{\mathrm{id}}^{!}$and $\underline{(\alpha \beta)^{!}}$follow from Remarks 7.6 and 7.8.

We need some preparations for Proposition 7.11.

Remark 7.10. Let $A$ be a k-algebra and $\alpha: Y \rightarrow X$ a separated, locally proper morphism of topological spaces with $\alpha_{!}: \operatorname{Mod}\left(Y_{A}\right) \rightarrow \operatorname{Mod}\left(X_{A}\right)$ of finite cohomological dimension. We claim that the evaluation of the 2-morphism

$$
\alpha_{!} \stackrel{\sim}{\rightarrow} \alpha_{!}^{A_{Y}}=\alpha_{!}\left(A_{Y} \otimes-\right) \stackrel{\alpha_{!}((7.2) \otimes(-))}{\longrightarrow} \alpha_{!}(\mathcal{L} \otimes-)=\alpha_{!}^{\mathcal{L}} .
$$

in $\mathrm{DGCAT}_{\mathrm{k}}$ at any componentwise injective object $J \in \underline{\mathrm{C}}\left(Y_{A}\right)$, and in particular at any object of $\underline{\mathbb{}}\left(Y_{A}\right)$, is a quasi-isomorphism. 
Let $J \in \underline{\mathrm{C}}\left(Y_{A}\right)$ be componentwise injective. Then the quasi-isomorphism (7.2) between h-flat objects gives rise to the quasi-isomorphism

$$
J \stackrel{\sim}{\rightarrow} A_{Y} \otimes J \stackrel{(7.2) \otimes \mathrm{id}}{\longrightarrow} \mathcal{L} \otimes J
$$

between complexes of $\alpha$ !-acyclic objects, by Proposition A.1 and [SS16, 5.3, Lemmas 5.5, 7.4]. Therefore applying $\alpha_{!}$preserves this quasi-isomorphism, by [SS16, Lemma 12.4.(b)]. This shows the claim.

Proposition 7.11. Let $A$ be a k-algebra and $\alpha: Y \rightarrow X$ a separated, locally proper morphism of topological spaces with $\alpha_{!}: \operatorname{Mod}\left(Y_{A}\right) \rightarrow \operatorname{Mod}\left(X_{A}\right)$ of finite cohomological dimension. Then the zig-zags

$$
\begin{gathered}
\underline{\alpha}_{!} \underline{\alpha}^{!}=\mathbf{i} \alpha_{!} \alpha_{\mathcal{L}}^{!} \frac{[\sim]}{\mathbf{i}(7.35) \alpha_{\mathcal{L}}^{!}} \mathbf{i} \alpha_{!}^{\mathcal{L}} \alpha_{\mathcal{L}}^{!} \rightarrow \mathbf{i} \stackrel{[\sim]}{\longleftarrow} \mathrm{id}, \\
\mathrm{id} \rightarrow \alpha_{\mathcal{L}}^{!} \mathbf{i} \alpha_{!}^{\mathcal{L}} \underset{\alpha_{\mathcal{L}}^{\prime} \mathbf{i}(7.35)}{\stackrel{[\sim]}{\longleftarrow}} \alpha_{\mathcal{L}}^{!} \mathbf{i} \alpha_{!}=\underline{\alpha}^{!} \underline{\alpha}_{!}
\end{gathered}
$$

of 2-morphisms in $\widetilde{\mathrm{ENH}}_{\mathrm{k}}$ where the first arrow in (7.38) is the composition id $\rightarrow$ $\alpha_{\mathcal{L}}^{!} \alpha_{!}^{\mathcal{L}} \rightarrow \alpha_{\mathcal{L}}^{!} \mathbf{i} \alpha_{!}^{\mathcal{L}}$ define ${ }^{2}$-morphisms

$$
\begin{aligned}
\underline{\alpha}_{!} \underline{\alpha}^{!} & \rightarrow \mathrm{id}, \\
\mathrm{id} & \rightarrow \underline{\alpha} \underline{\underline{\alpha}} \underline{ }
\end{aligned}
$$

in $\mathrm{ENH}_{\mathrm{k}}$ that enhance the counit $\left(\mathbf{R} \alpha_{!}\right) \alpha^{!} \rightarrow$ id and the unit id $\rightarrow \alpha^{!}\left(\mathbf{R} \alpha_{!}\right)$of the adjunction $\left(\mathbf{R} \alpha !, \alpha^{!}\right)$.

The first arrow in (7.38) is not expanded as the given composition since the $\mathcal{K}$ functor $\alpha_{\mathcal{L}}^{!} \alpha_{!}^{\mathcal{L}}$ does not land in $\underline{\mathbb{I}}\left(Y_{A}\right)$ in general.

Proof. Note that $\mathbf{i}(7.35): \mathbf{i} \alpha_{!} \rightarrow \mathbf{i} \alpha_{!}^{\mathcal{L}}$ considered as a 2-morphism between 1-morphisms $\underline{\mathbb{I}}\left(Y_{A}\right) \rightarrow \underline{\mathbb{I}}\left(X_{A}\right)$ in $\widetilde{\mathrm{ENH}}_{\mathrm{k}}$ is an objectwise homotopy equivalence, by Remark 7.10. We leave the rest of the proof to the reader.

Proposition 7.12. Let $A$ be a k-algebra and

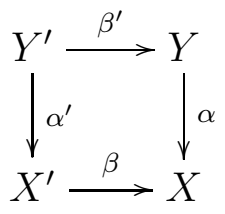

a cartesian diagram of topological spaces with a separated, locally proper, and with $\alpha_{!}: \operatorname{Mod}\left(Y_{A}\right) \rightarrow \operatorname{Mod}\left(X_{A}\right)$ of finite cohomological dimension. Let $t: \beta^{-1} \alpha_{!} \stackrel{\sim}{\rightarrow} \alpha_{!}^{\prime} \beta^{\prime-1}$ be the isomorphism obtained from [SS16, Thm. 4.4]. Then the zig-zag

$$
\underline{\beta}^{-1} \underline{\alpha}_{!}=\mathbf{i} \beta^{-1} \mathbf{i} \alpha_{!} \stackrel{[\sim]}{\longleftarrow} \mathbf{i} \beta^{-1} \alpha_{!} \stackrel{\mathbf{i} t}{\sim} \mathbf{i} \alpha_{!}^{\prime} \beta^{\prime-1} \stackrel{[\sim]}{\longrightarrow} \mathbf{i} \alpha_{!}^{\prime} \mathbf{i} \beta^{\prime-1}=\underline{\alpha}_{!}^{\prime} \underline{\beta}^{\prime-1}
$$


of objectwise homotopy equivalences in $\widetilde{\mathrm{ENH}}_{\mathrm{k}}$ defines a 2-isomorphism

$$
\underline{\beta}^{-1} \underline{\alpha}_{!} \stackrel{\sim}{\rightarrow} \underline{\alpha}_{!}^{\prime} \underline{\beta}^{\prime-1}
$$

in $\mathrm{ENH}_{\mathrm{k}}$ that enhances the derived proper base change isomorphism

$$
\mathbf{L} \beta^{-1} \mathbf{R} \alpha_{!} \stackrel{\sim}{\rightarrow} \mathbf{R} \alpha_{!}^{\prime} \mathbf{L} \beta^{\prime-1}
$$

from [SS16, Thm. 8.3.(c)].

Proof. We can assume that $\mathbf{L} \beta^{-1} E=\beta^{-1} E$ and $\mathbf{R} \alpha_{!} F=\alpha_{!} \mathbf{i} F$, for $E \in \mathrm{D}\left(X_{A}\right)$ and $F \in \mathrm{D}\left(Y_{A}\right)$, and similarly for $\mathbf{L} \beta^{\prime-1}$ and $\mathbf{R} \alpha_{!}^{\prime}$. Then $\mathbf{L} \beta^{-1} \mathbf{R} \alpha_{!} F \stackrel{\sim}{\rightarrow} \mathbf{R} \alpha_{!}^{\prime} \mathbf{L} \beta^{\prime-1} F$ is obtained from the zig-zag

$$
\beta^{-1} \alpha_{!} \mathbf{i} F \stackrel{t \mathbf{i}}{\longrightarrow} \alpha_{!}^{\prime} \beta^{\prime-1} \mathbf{i} F \stackrel{\alpha_{!}^{\prime} \iota_{\beta^{\prime}-1} \mathbf{i} F}{\longrightarrow} \alpha_{!}^{\prime} \mathbf{i} \beta^{\prime-1} \mathbf{i} F \stackrel{\alpha_{!}^{\prime} \mathbf{i} \beta^{\prime-1} \iota_{F}}{\stackrel{1}{\sim}} \alpha_{!}^{\prime} \mathbf{i} \beta^{\prime-1} F
$$

of quasi-isomorphisms in $\mathrm{C}\left(X_{A}^{\prime}\right)$; this follows from [SS16, 8.3] which also shows that the third arrow in (7.42) is an objectwise homotopy equivalence. The rest of the proof is obvious and left to the reader.

Proposition 7.13. Let $A$ be a k-algebra and $\alpha: Y \rightarrow X$ a separated, locally proper morphism of topological spaces with $\alpha_{!}: \operatorname{Mod}\left(Y_{A}\right) \rightarrow \operatorname{Mod}\left(X_{A}\right)$ of finite cohomological dimension. Consider the composition

$$
\alpha_{!}(-) \otimes(-) \rightarrow \alpha_{!}\left((-) \otimes \alpha^{-1}(-)\right) \stackrel{\alpha_{!} \iota\left((-) \otimes \alpha^{-1}(-)\right)}{\longrightarrow} \alpha_{!} \mathbf{i}\left((-) \otimes \alpha^{-1}(-)\right)
$$

where the first arrow is obtained from [SS16, 6.1]. This composition gives rise to the third arrow in the zig-zag

$$
\begin{aligned}
& \underline{\alpha}_{!}(-) \underline{\otimes}(-)=\mathbf{i}\left(\mathbf{e i} \alpha_{!}(-) \otimes \mathbf{e}(-)\right) \stackrel{[\sim]}{\longrightarrow} \mathbf{i}\left(\mathbf{i} \alpha_{!}(-) \otimes \mathbf{e}(-)\right) \stackrel{[\sim]}{\longleftarrow} \mathbf{i}\left(\alpha_{!}(-) \otimes \mathbf{e}(-)\right) \\
& \stackrel{[\sim]}{\longrightarrow} \mathbf{i} \alpha_{!} \mathbf{i}\left((-) \otimes \alpha^{-1} \mathbf{e}(-)\right) \stackrel{[\sim]}{\longleftarrow} \mathbf{i} \alpha_{!} \mathbf{i}\left(\mathbf{e}(-) \otimes \alpha^{-1} \mathbf{e}(-)\right) \stackrel{[\sim]}{\longrightarrow} \mathbf{i} \alpha_{!} \mathbf{i}\left(\mathbf{e}(-) \otimes \alpha^{-1}(-)\right) \\
& \stackrel{[\sim]}{\longrightarrow} \mathbf{i} \alpha_{!} \mathbf{i}\left(\mathbf{e}(-) \otimes \mathbf{i} \alpha^{-1}(-)\right) \stackrel{[\sim]}{\longleftarrow} \mathbf{i} \alpha_{!} \mathbf{i}\left(\mathbf{e}(-) \otimes \mathbf{e i} \alpha^{-1}(-)\right)=\underline{\alpha}_{!}\left((-) \otimes \underline{\alpha}^{-1}(-)\right)
\end{aligned}
$$

of objectwise homotopy equivalences in $\widetilde{\mathrm{ENH}}_{\mathrm{k}}$ and defines a 2-isomorphism

$$
\underline{\alpha}_{!}(-) \underline{\otimes}(-) \stackrel{\sim}{\rightarrow} \underline{\alpha}_{!}\left((-) \underline{\otimes} \underline{\alpha}^{-1}(-)\right)
$$

in $\mathrm{ENH}_{\mathrm{k}}$ that enhances the isomorphism (the derived projection formula)

$$
\mathbf{R} \alpha_{!}(-) \otimes^{\mathbf{L}}(-) \stackrel{\sim}{\rightarrow} \mathbf{R} \alpha_{!}\left((-) \otimes^{\mathbf{L}} \mathbf{L} \alpha^{-1}(-)\right)
$$

from [SS16, Thm. 8.3.(d)].

Proof. Let $S \in \mathrm{C}\left(Y_{A}\right)$ have $\alpha$-c-soft components and let $F \in \mathrm{C}\left(X_{A}\right)$ be h-flat. We claim that the evaluation of the composition of $(7.46)$ at $(S, F)$ is a quasi-isomorphism. 
By [Spa88, Prop. 5.6] and with the notation from there, there is a quasi-isomorphism $P \rightarrow F$ with $P \in \underset{\mathfrak{P}}{\rightarrow}\left(X_{A}\right)$. Consider the commutative diagram

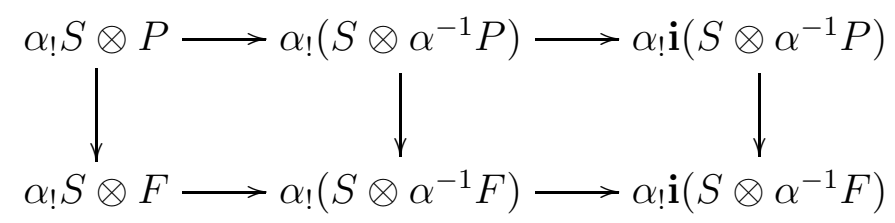

whose rows come from (7.46). The proof of [SS16, Thm. 8.3.(d)] shows that the upper left horizontal arrow is an isomorphism and that the upper right horizontal arrow is a quasi-isomorphism. The left and the right vertical arrows are quasi-isomorphisms because $F, P, \alpha^{-1} F, \alpha^{-1} P$ are h-flat. Hence the composition of the two lower horizontal arrows is a quasi-isomorphism. This proves our claim.

This claim and the fact that $\mathbb{I}$-fibrant objects have injective and hence $\alpha$-c-soft components imply that the third arrow in (7.47) is an objectwise homotopy equivalence.

We can assume that $\mathbf{L} \alpha^{-1} E=\alpha^{-1} E$ and $\mathbf{R} \alpha_{!} G=\alpha_{!} \mathbf{i} G$, for $E \in \mathrm{D}\left(X_{A}\right)$ and $G \in \mathrm{D}\left(Y_{A}\right)$, and that $\left(-\otimes^{\mathbf{L}}-\right)=(-\otimes \mathbf{e}(-))$. Then $(7.49)$ evaluated at $(G, E)$ is obtained from the zig-zag

$$
\begin{aligned}
\alpha_{!} \mathbf{i} G \otimes \mathbf{e} E \stackrel{(7.46)_{(\mathbf{i} G, \mathbf{e} E)}}{\longrightarrow} \alpha_{!} \mathbf{i}\left(\mathbf{i} G \otimes \alpha^{-1} \mathbf{e} E\right) & \leftarrow \alpha_{!} \mathbf{i}\left(\mathbf{i} G \otimes \mathbf{e} \alpha^{-1} \mathbf{e} E\right) \\
& \leftarrow \alpha_{!} \mathbf{i}\left(G \otimes \mathbf{e} \alpha^{-1} \mathbf{e} E\right) \rightarrow \alpha_{!} \mathbf{i}\left(G \otimes \mathbf{e} \alpha^{-1} E\right)
\end{aligned}
$$

of quasi-isomorphisms in $\mathrm{C}\left(X_{A}\right)$ (the first arrow is a quasi-isomorphism by the above claim, and the second one because $\alpha^{-1} \mathbf{e} E$ is h-flat), as follows from [SS16, 8.3]. The rest of the proof is obvious and left to the reader.

Our next aim is Proposition 7.16.

Let $A$ be a k-algebra and $\alpha: Y \rightarrow X$ a separated, locally proper morphism of topological spaces. The map constructed in [SS16, 6.1] gives rise to a 2-morphism

$$
\alpha_{!}(-) \otimes(-) \rightarrow \alpha_{!}\left((-) \otimes \alpha^{-1}(-)\right)
$$

between 1-morphisms $\left(\underline{\mathrm{C}}\left(Y_{A}\right), \underline{\mathrm{C}}\left(X_{A}\right)\right) \rightarrow \underline{\mathrm{C}}\left(X_{A}\right)$ in $\mathrm{DGCAT}_{\mathrm{k}}$.

Lemma 7.14. Let $A$ be a k-algebra and $\alpha: Y \rightarrow X$ a separated, locally proper morphism of topological spaces. Let $F \in \mathrm{C}\left(X_{A}\right)$ be componentwise flat and $T \in \mathrm{C}\left(Y_{A}\right)$. Then the evaluation of $(7.52)$ at $(T, F)$ is an isomorphism

$$
(\alpha ! T) \otimes F \stackrel{\sim}{\rightarrow} \alpha_{!}\left(T \otimes \alpha^{-1} F\right)
$$

Proof. From [SS16, Thm. 6.2, Lemma 4.6] we obtain the following two statements. For $p, q \in \mathbb{Z}$ the morphism $\left(\alpha_{!} T^{p}\right) \otimes F^{q} \stackrel{\sim}{\rightarrow} \alpha_{!}\left(T^{p} \otimes \alpha^{-1} F^{q}\right)$ is an isomorphism. For fixed $n \in \mathbb{N}$, the morphism $\bigoplus_{p+q=n} \alpha_{!}\left(T^{p} \otimes \alpha^{-1} F^{q}\right) \stackrel{\sim}{\rightarrow} \alpha_{!}\left(\bigoplus_{p+q=n} T^{p} \otimes \alpha^{-1} F^{q}\right)$ is an isomorphism. The lemma follows. 
Assume in addition that $\alpha_{!}: \operatorname{Mod}\left(Y_{A}\right) \rightarrow \operatorname{Mod}\left(X_{A}\right)$ has finite cohomological dimension. From (7.52) and symmetry we obtain 2-morphisms

$$
\begin{gathered}
\alpha_{!}^{\mathcal{L}}(-) \otimes(-) \rightarrow \alpha_{!}^{\mathcal{L}}\left((-) \otimes \alpha^{-1}(-)\right), \\
(-) \otimes \alpha_{!}^{\mathcal{L}}(-) \rightarrow \alpha_{!}^{\mathcal{L}}\left(\alpha^{-1}(-) \otimes(-)\right)
\end{gathered}
$$

between 1-morphisms $\left(\underline{\mathrm{C}}\left(Y_{A}\right), \underline{\mathrm{C}}\left(X_{A}\right)\right) \rightarrow \underline{\mathrm{C}}\left(X_{A}\right)$ and $\left(\underline{\mathrm{C}}\left(X_{A}\right), \underline{\mathrm{C}}\left(Y_{A}\right)\right) \rightarrow \underline{\mathrm{C}}\left(X_{A}\right)$ in DGCAT $_{\mathrm{k}}$, respectively.

Let $F, I \in \mathrm{C}\left(X_{A}\right)$ and $J \in \mathrm{C}\left(Y_{A}\right)$. The evaluation of the 2-morphism (7.55) at $(F, J)$, the adjunctions $\left(\alpha^{-1}, \alpha_{*}\right)$ and $\left(\alpha_{!}^{\mathcal{L}}, \alpha_{\mathcal{L}}^{!}\right)$and the $\otimes$-Hom-adjunction yield morphisms

$$
\begin{aligned}
\underline{\mathrm{C}}_{X_{A}}\left(F, \alpha_{*} \mathcal{H o m}\left(J, \alpha_{\mathcal{L}}^{!} I\right)\right) & \sim \underline{\mathrm{C}}_{Y_{A}}\left(\alpha^{-1} F, \mathcal{H o m}\left(J, \alpha_{\mathcal{L}}^{!} I\right)\right) \\
& \sim \underline{\mathrm{C}}_{Y_{A}}\left(\alpha^{-1}(F) \otimes J, \alpha_{\mathcal{L}}^{!} I\right) \stackrel{\sim}{\leftarrow} \underline{\mathrm{C}}_{X_{A}}\left(\alpha_{!}^{\mathcal{L}}\left(\alpha^{-1}(F) \otimes J\right), I\right) \\
& \stackrel{(7.55)_{(F, J)}^{*}}{\longrightarrow} \underline{\mathrm{C}}_{X_{A}}\left(F \otimes \alpha_{!}^{\mathcal{L}}(J), I\right) \stackrel{\sim}{\rightarrow} \underline{\mathrm{C}}_{X_{A}}\left(F, \mathcal{H o m}\left(\alpha_{!}^{\mathcal{L}}(J), I\right)\right)
\end{aligned}
$$

in $\mathcal{K}$. Hence the Yoneda lemma (or, more concretely, evaluating at $F=v_{!}\left(A_{V}\right)$, for $v: V \rightarrow Y$ the embedding of an open subset $V$ of $Y$, for varying $V$ ) and naturality in $J$ and $I$ yield a 2-morphism

$$
\alpha_{*} \mathcal{H o m}\left(-, \alpha_{\mathcal{L}}^{!}(-)\right) \rightarrow \mathcal{H o m}\left(\alpha_{!}^{\mathcal{L}}(-),-\right)
$$

between 1-morphisms $\left(\underline{\mathrm{C}}\left(Y_{A}\right)^{\mathrm{op}}, \underline{\mathrm{C}}\left(X_{A}\right)\right) \rightarrow \underline{\mathrm{C}}\left(X_{A}\right)$ in $\mathrm{DGCAT}_{\mathrm{k}}$.

Lemma 7.15. Let $A$ be a k-algebra and $\alpha: Y \rightarrow X$ a separated, locally proper morphism of topological spaces with $\alpha_{!}: \operatorname{Mod}\left(Y_{A}\right) \rightarrow \operatorname{Mod}\left(X_{A}\right)$ of finite cohomological dimension. Then the 2-morphisms (7.54), (7.55) and (7.57) are 2-isomorphisms.

Proof. In order to show that $(7.57)$ is a 2-isomorphism we need to show that the evaluation

$$
\alpha_{*} \mathcal{H o m}\left(J, \alpha_{\mathcal{L}}^{!} I\right) \rightarrow \mathcal{H o m}\left(\alpha_{!}^{\mathcal{L}} J, I\right) .
$$

of (7.57) at $J \in \underline{\mathrm{C}}\left(Y_{A}\right)$ and $I \in \underline{\mathrm{C}}\left(X_{A}\right)$ is an isomorphism. Let $F \in \underline{\mathrm{C}}\left(X_{A}\right)$ be componentwise flat. Then $\underline{\mathrm{C}}(F,-)$ applied to $(7.58)$ is an isomorphism because all arrows in (7.56) are isomorphisms, by Lemma 7.14. Let $v: V \subset Y$ be the embedding of an arbitrary open subset $V$ of $Y$ and take $F=v_{!}\left(A_{V}\right)$. This shows that (7.58) evaluated at $V$ is an isomorphism. Hence (7.57) is a 2-isomorphism.

If $F \in \underline{\mathrm{C}}\left(X_{A}\right)$ is now an arbitrary object, we deduce that all morphisms in (7.56) are in fact isomorphisms. Hence the Yoneda lemma implies that (7.55) is a 2-isomorphism. By symmetry, (7.54) is also a 2-isomorphism. (The statement that (7.55) is a 2isomorphism can also be proved directly: its evaluation at a componentwise flat object $F$ and an arbitrary object $J$ is an isomorphism, and an arbitrary object $G \in \mathrm{C}\left(X_{A}\right)$ admits a short exact sequence $F^{\prime} \rightarrow F \rightarrow G \rightarrow 0$ in $\mathrm{C}\left(X_{A}\right)$ where $F$ and $F^{\prime}$ have flat components.) 
Proposition 7.16. Let $A$ be a k-algebra and $\alpha: Y \rightarrow X$ a separated, locally proper morphism of topological spaces with $\alpha_{!}: \operatorname{Mod}\left(Y_{A}\right) \rightarrow \operatorname{Mod}\left(X_{A}\right)$ of finite cohomological dimension. Then the zig-zag

$$
\begin{aligned}
& \underline{\alpha}_{*} \underline{\mathcal{H} o m}\left(-, \underline{\alpha}^{!}(-)\right)=\mathbf{i} \alpha_{*} \mathbf{i} \mathcal{H} \operatorname{Hom}\left(-, \alpha_{\mathcal{L}}^{!}(-)\right) \stackrel{[\sim]}{\longleftarrow} \mathbf{i} \alpha_{*} \mathcal{H} \operatorname{Hom}\left(-, \alpha_{\mathcal{L}}^{!}(-)\right) \\
& \stackrel{\mathbf{i}(7.57)}{\sim} \mathbf{i} \mathcal{H} o m\left(\alpha_{!}^{\mathcal{L}}(-),-\right) \stackrel{[\sim]}{\longleftarrow} \mathbf{i} \mathcal{H} o m\left(\mathbf{i} \alpha_{!}^{\mathcal{L}}(-),-\right)=\mathbf{i} \mathcal{H} o m(\mathbf{i} \alpha !(\mathcal{L} \otimes-),-) \\
& \stackrel{[\sim]}{\longrightarrow} \mathbf{i} \mathcal{H} o m\left(\mathbf{i} \alpha_{!}\left(A_{Y} \otimes-\right),-\right) \stackrel{\sim}{\leftarrow} \mathcal{H} \operatorname{Hom}\left(\mathbf{i} \alpha_{!}(-),-\right)=\underline{\mathcal{H} o m}\left(\underline{\alpha_{!}}(-),-\right)
\end{aligned}
$$

of objectwise homotopy equivalences in $\widetilde{\mathrm{ENH}}_{\mathrm{k}}$ defines a 2-isomorphism

$$
\underline{\alpha}_{*} \underline{\mathcal{H} o m}\left(-, \underline{\alpha}^{!}(-)\right) \stackrel{\sim}{\rightarrow} \underline{\mathcal{H} o m}\left(\underline{\alpha}_{!}(-),-\right)
$$

in $\mathrm{ENH}_{\mathrm{k}}$ that enhances the 2-isomorphism

$$
\mathbf{R} \alpha_{*} \mathbf{R} \mathcal{H o m}\left(-, \alpha^{!}(-)\right) \stackrel{\sim}{\rightarrow} \mathbf{R} \mathcal{H o m}\left(\mathbf{R} \alpha_{!}(-),-\right)
$$

from [SS16, Thm. 8.3.(d)].

Proof. If we evaluate (7.59) at $J \in \mathbb{I}\left(Y_{A}\right)$ and $I \in \mathbb{I}\left(X_{A}\right)$ the first arrow is a homotopy equivalence by Propositions A.6, A.9 and h-injectivity of $\alpha_{\mathcal{L}}^{!} I$. The second arrow is an isomorphism by Lemma 7.15, the third arrow is obviously a homotopy equivalence. The fourth arrow is a homotopy equivalence because $J \cong A_{Y} \otimes J \rightarrow \mathcal{L} \otimes J$ is a quasiisomorphism between componentwise $\alpha$-c-soft complexes, by Proposition A.1 and [SS16, 5.3, Lemma 7.4], and hence $\alpha_{!}\left(A_{Y} \otimes J\right) \rightarrow \alpha_{!}(\mathcal{L} \otimes J)$ is a quasi-isomorphism by [SS16, Lemma 12.4.(b)]. The fifth arrow clearly is an isomorphism.

For $F, I \in \mathrm{C}\left(X_{A}\right)$ and $J \in \mathrm{C}\left(Y_{A}\right)$, all arrows in (7.56) are isomorphisms, by Lemma 7.15. Taking the 0 -th cohomology of this sequence of isomorphisms gives isomorphisms

$$
\begin{aligned}
{\left[\underline{\mathrm{C}}_{X_{A}}\right]\left(F, \alpha_{*} \mathcal{H o m}\left(J, \alpha_{\mathcal{L}}^{!} I\right)\right) } & \stackrel{\sim}{\leftarrow}\left[\underline{\mathrm{C}}_{Y_{A}}\right]\left(\alpha^{-1} F, \mathcal{H o m}\left(J, \alpha_{\mathcal{L}}^{!} I\right)\right) \\
\stackrel{\sim}{\sim}\left[\underline{\mathrm{C}}_{Y_{A}}\right]\left(\alpha^{-1}(F) \otimes J, \alpha_{\mathcal{L}} I\right) & \sim\left[\underline{\mathrm{C}}_{X_{A}}\right]\left(\alpha_{!}^{\mathcal{L}}\left(\alpha^{-1}(F) \otimes J\right), I\right) \\
& \stackrel{(7.55)_{(F, J)}^{*}}{\sim}\left[\underline{\mathrm{C}}_{X_{A}}\right]\left(F \otimes \alpha_{!}^{\mathcal{L}}(J), I\right) \stackrel{\sim}{\rightarrow}\left[\underline{\mathrm{C}}_{X_{A}}\right]\left(F, \mathcal{H o m}\left(\alpha_{!}^{\mathcal{L}}(J), I\right)\right) .
\end{aligned}
$$

Now assume that $F$ is h-flat and that $I$ is h-injective. Then all objects in this sequence are spaces of morphisms in either $\left[\underline{\mathrm{C}}\left(Y_{A}\right)\right]$ or $\left[\underline{\mathrm{C}}\left(X_{A}\right)\right]$ which have either h-injective target or h-flat source and weakly h-injective target: this follows from Propositions A.2, A.6, A.9 and the fact that $\alpha_{\mathcal{L}}^{!}$preserves h-injectives. Therefore, by Corollary A.11, all these morphism spaces map isomorphically to the corresponding morphism spaces in the derived categories $\mathrm{D}\left(Y_{A}\right)$ and $\mathrm{D}\left(X_{A}\right)$, respectively. We can moreover assume that $\mathbf{R} \mathcal{H}$ om (resp. $\otimes^{\mathbf{L}}$ ) is computed naively if its second argument is h-injective (resp. if one of its arguments is h-flat), that $\mathbf{L} \alpha^{-1}$ is computed naively, that $\mathbf{R} \alpha_{*}$ is computed naively if its argument is weakly h-injective, that $\alpha^{!}=\mathbf{R} \alpha_{\mathcal{L}}^{!}$is 
computed naively if its argument is h-injective, and that $\mathbf{R} \alpha_{!}=\alpha_{!}^{\mathcal{L}}$ (by the proof of [SS16, Thm. 8.3.(a)]). Hence we get a sequence of isomorphisms

$$
\begin{aligned}
& \mathrm{D}_{X_{A}}\left(F, \mathbf{R} \alpha_{*} \mathbf{R H o m}(J, \alpha ! I)\right) \sim \mathrm{D}_{Y_{A}}\left(\mathbf{L} \alpha^{-1} F, \mathbf{R H o m}(J, \alpha ! I)\right) \\
& \stackrel{\sim}{\leftarrow} \mathrm{D}_{Y_{A}}\left(\mathbf{L} \alpha^{-1}(F) \otimes \mathbf{L} J, \alpha ! I\right) \sim \mathrm{D}_{X_{A}}\left(\mathbf{R} \alpha_{!}\left(\mathbf{L} \alpha^{-1}(F) \otimes \mathbf{L} J\right), I\right) \\
& \stackrel{\sim}{\rightarrow} \mathrm{D}_{X_{A}}\left(F \otimes^{\mathbf{L}} \mathbf{R} \alpha_{!}(J), I\right) \stackrel{\sim}{\rightarrow} \mathrm{D}_{X_{A}}\left(F, \mathbf{R H o m}\left(\mathbf{R} \alpha_{!}(J), I\right)\right)
\end{aligned}
$$

which combines with (7.62) to a commutative diagram in the obvious way. Moreover, the isomorphisms in (7.63) are the isomorphisms obtained from the adjunctions $\left(\mathbf{L} \alpha^{-1}, \mathbf{R} \alpha_{*}\right)$ and $\left(\mathbf{R} \alpha_{!}, \alpha^{!}\right)$, the $\otimes$-Hom-adjunction, and the derived projection formula (7.49). Now recall that (7.61) is by definition the isomorphism giving rise to the composition in (7.63). The rest of the proof is left to the reader.

Let $A$ be a k-algebra and $\alpha: Y \rightarrow X$ a separated, locally proper morphism of topological spaces with $\alpha_{!}: \operatorname{Mod}\left(Y_{A}\right) \rightarrow \operatorname{Mod}\left(X_{A}\right)$ of finite cohomological dimension. Let $T \in \mathrm{C}\left(Y_{A}\right)$ and $F, J \in \mathrm{C}\left(X_{A}\right)$. The evaluation of the 2-isomorphism (7.54) (see Lemma 7.15) at $(T, F)$, the adjunctions $\left(\alpha_{!}^{\mathcal{L}}, \alpha_{\mathcal{L}}^{!}\right)$and the $\otimes$-Hom-adjunction yield isomorphisms

$$
\begin{aligned}
& \underline{\mathrm{C}}_{Y_{A}}\left(T, \mathcal{H} o m\left(\alpha^{-1} F, \alpha_{\mathcal{L}}^{!} J\right)\right) \stackrel{\sim}{\leftarrow} \underline{\mathrm{C}}_{Y_{A}}\left(T \otimes \alpha^{-1} F, \alpha_{\mathcal{L}} J\right) \\
& \stackrel{\sim}{\leftarrow} \underline{\mathrm{C}}_{X_{A}}\left(\alpha_{!}^{\mathcal{L}}\left(T \otimes \alpha^{-1} F\right), J\right) \stackrel{(7.54)_{(T, F)}^{*}}{\sim} \underline{\mathrm{C}}_{X_{A}}\left(\alpha_{!}^{\mathcal{L}}(T) \otimes F, J\right) \\
& \stackrel{\sim}{\rightarrow} \underline{\mathrm{C}}_{X_{A}}\left(\alpha_{!}^{\mathcal{L}}(T), \mathcal{H o m}(F, J)\right) \stackrel{\sim}{\rightarrow} \underline{\mathrm{C}}_{Y_{A}}\left(T, \alpha_{\mathcal{L}}^{!} \mathcal{H o m}(F, J)\right)
\end{aligned}
$$

in $\mathcal{K}$. Hence the Yoneda lemma (or, more concretely, evaluating at $T=v_{!}\left(A_{V}\right)$ for $v: V \rightarrow Y$ the embedding of an open subset $V$ of $Y$, for varying $V$ ) and naturality in $F$ and $J$ yield a 2-isomorphism

$$
\mathcal{H o m}\left(\alpha^{-1}(-), \alpha_{\mathcal{L}}^{!}(-)\right) \stackrel{\sim}{\rightarrow} \alpha_{\mathcal{L}}^{!} \mathcal{H o m}(-,-)
$$

between 1-morphisms $\left(\underline{\mathrm{C}}\left(X_{A}\right), \underline{\mathrm{C}}\left(X_{A}\right)\right) \rightarrow \underline{\mathrm{C}}\left(Y_{A}\right)$ in $\mathrm{DGCAT}_{\mathrm{k}}$.

Proposition 7.17. Let $A$ be a k-algebra and $\alpha: Y \rightarrow X$ a separated, locally proper morphism of topological spaces with $\alpha_{!}: \operatorname{Mod}\left(Y_{A}\right) \rightarrow \operatorname{Mod}\left(X_{A}\right)$ of finite cohomological dimension. Then the zig-zag

$$
\begin{aligned}
& \underline{\mathcal{H} o m}\left(\underline{\alpha}^{-1}(-), \underline{\alpha}^{!}(-)\right)=\mathbf{i} \mathcal{H} o m\left(\mathbf{i} \alpha^{-1}(-), \alpha_{\mathcal{L}}^{!}(-)\right) \stackrel{[\sim]}{\longrightarrow} \mathbf{i} \mathcal{H} o m\left(\alpha^{-1}(-), \alpha_{\mathcal{L}}^{!}(-)\right) \\
& \stackrel{[\sim]}{\longrightarrow} \mathbf{i} \mathcal{H o m}\left(\alpha^{-1} \mathbf{e}(-), \alpha_{\mathcal{L}}^{!}(-)\right) \stackrel{\mathbf{i}(7.65)(\mathbf{e}(-),-)}{\sim} \mathbf{i} \alpha_{\mathcal{L}}^{!} \mathcal{H} o m(\mathbf{e}(-),-) \\
& \stackrel{[\sim]}{\longrightarrow} \mathbf{i} \alpha_{\mathcal{L}}^{!} \mathbf{i} \mathcal{H} \operatorname{Hom}(\mathbf{e}(-),-) \stackrel{[\sim]}{\longleftarrow} \alpha_{\mathcal{L}}^{!} \mathbf{i} \mathcal{H o m}(\mathbf{e}(-),-) \\
& \stackrel{[\sim]}{\longleftarrow} \alpha_{\mathcal{L}}^{!} \mathbf{i} \mathcal{H} \operatorname{lom}(-,-)=\underline{\alpha} \underline{\underline{\mathcal{H} o m}}(-,-)
\end{aligned}
$$


of objectwise homotopy equivalences in $\widetilde{\mathrm{ENH}}_{\mathrm{k}}$ defines a 2-isomorphism

$$
\underline{\mathcal{H} o m}\left(\underline{\alpha}^{-1}(-), \underline{\alpha}^{!}(-)\right) \stackrel{\sim}{\rightarrow} \underline{\alpha^{!}} \underline{\mathcal{H} o m}(-,-)
$$

in $\mathrm{ENH}_{\mathrm{k}}$ that enhances the 2-isomorphism

$$
\mathbf{R} \mathcal{H o m}\left(\mathbf{L} \alpha^{-1}(-), \alpha^{!}(-)\right) \stackrel{\sim}{\rightarrow} \alpha ! \mathbf{R} \mathcal{H} o m(-,-)
$$

from [SS16, Thm. 8.3.(d)].

Proof. If we evaluate (7.66) at $I, J \in \mathbb{I}\left(X_{A}\right)$ the first two arrows are homotopy equivalences because $\alpha_{\mathcal{L}}^{!}$preserves h-injectives (and I-fibrant objects), the third arrow is an isomorphism because we have seen above that $(7.65)$ is an isomorphism, the fourth arrow is a homotopy equivalence because $\mathcal{H o m}(\mathbf{e} I, J)$ is h-injective by Proposition A.6, the fifth arrow is a homotopy equivalence because $\alpha_{\mathcal{L}}^{!}$preserves h-injectives and the sixth arrow is obviously a homotopy equivalence. Hence we get the 2 -isomorphism (7.67).

For $T \in \mathrm{C}\left(Y_{A}\right)$ and $F, J \in \mathrm{C}\left(X_{A}\right)$, consider the sequence (7.64) of isomorphisms. Taking the 0 -th cohomology of this sequence gives isomorphisms

$$
\begin{aligned}
{\left[\underline{\mathrm{C}}_{Y_{A}}\right] } & \left(T, \mathcal{H o m}\left(\alpha^{-1} F, \alpha_{\mathcal{L}}^{!} J\right)\right) \stackrel{\sim}{\leftarrow}\left[\underline{\mathrm{C}}_{Y_{A}}\right]\left(T \otimes \alpha^{-1} F, \alpha_{\mathcal{L}}^{!} J\right) \\
& \sim\left[\underline{\mathrm{C}}_{X_{A}}\right]\left(\alpha_{!}^{\mathcal{L}}\left(T \otimes \alpha^{-1} F\right), J\right) \stackrel{(7.54)_{(T, F)}^{*}}{\sim}\left[\underline{\mathrm{C}}_{X_{A}}\right]\left(\alpha_{!}^{\mathcal{L}}(T) \otimes F, J\right) \\
& \stackrel{\sim}{\rightarrow}\left[\underline{\mathrm{C}}_{X_{A}}\right]\left(\alpha_{!}^{\mathcal{L}}(T), \mathcal{H o m}(F, J)\right) \stackrel{\sim}{\rightarrow}\left[\underline{\mathrm{C}}_{Y_{A}}\right]\left(T, \alpha_{\mathcal{L}}^{!} \mathcal{H o m}(F, J)\right) .
\end{aligned}
$$

Now assume that $F$ is h-flat and that $J$ is h-injective. Then all objects in this sequence are spaces of morphisms in either $\left[\underline{\mathrm{C}}\left(Y_{A}\right)\right]$ or $\left[\underline{\mathrm{C}}\left(X_{A}\right)\right]$ whose target objects are h-injective: this follows from Propositions A.2, A.6 and the fact that $\alpha_{\mathcal{L}}^{!}$preserves h-injectives. Therefore, all these morphism spaces map isomorphically to the corresponding morphism spaces in the derived categories $\mathrm{D}\left(Y_{A}\right)$ and $\mathrm{D}\left(X_{A}\right)$, respectively. We can moreover assume that RHom (resp. $\otimes^{\mathbf{L}}$ ) is computed naively if its second argument is h-injective (resp. if one of its arguments is h-flat), that $\mathbf{L} \alpha^{-1}$ is computed naively, that $\alpha^{!}=\mathbf{R} \alpha_{\mathcal{L}}^{!}$is computed naively if its argument is h-injective, and that $\mathbf{R} \alpha_{!}=\alpha_{!}^{\mathcal{L}}$. Hence we get a sequence of isomorphisms

$$
\begin{aligned}
& \mathrm{D}_{Y_{A}}(T,\left.\mathbf{R H o m}\left(\mathbf{L} \alpha^{-1} F, \alpha^{!} J\right)\right) \stackrel{\sim}{\sim} \mathrm{D}_{Y_{A}}\left(T \otimes^{\mathbf{L}} \mathbf{L} \alpha^{-1} F, \alpha^{!} J\right) \\
& \stackrel{\sim}{\leftarrow} \mathrm{D}_{X_{A}}\left(\mathbf{R} \alpha_{!}\left(T \otimes^{\mathbf{L}} \mathbf{L} \alpha^{-1} F\right), J\right) \stackrel{\sim}{\rightarrow} \mathrm{D}_{X_{A}}\left(\left(\mathbf{R} \alpha_{!} T\right) \otimes \mathbf{L} F, J\right) \\
& \stackrel{\sim}{\rightarrow} \mathrm{D}_{X_{A}}(\mathbf{R} \alpha ! T, \mathbf{R} \mathcal{H o m}(F, J)) \stackrel{\sim}{\rightarrow} \mathrm{D}_{Y_{A}}(T, \alpha \mathbf{R} \mathcal{H} \operatorname{om}(F, J))
\end{aligned}
$$

which combines with (7.69) to a commutative diagram in the obvious way. Moreover, the isomorphisms in (7.70) are the isomorphisms obtained from the $\otimes$ - $\mathcal{H}$ omadjunction, the adjunction $\left(\mathbf{R} \alpha_{!}, \alpha^{!}\right)$, and the derived projection formula (7.49). Now recall that (7.68) is by definition the isomorphism giving rise to the composition in (7.70). The rest of the proof is left to the reader. 
7.5.2. Lifts of commutative diagrams.

Proposition 7.18. Let $A$ be a k-algebra and $\alpha: Y \rightarrow X$ a separated, locally proper morphism of topological spaces with $\alpha_{!}: \operatorname{Mod}\left(Y_{A}\right) \rightarrow \operatorname{Mod}\left(X_{A}\right)$ of finite cohomological dimension. Then the two 1-morphisms $\underline{\alpha}_{\text {! }}$ and $\underline{\alpha}^{!}$and the two 2-morphisms (7.40) and (7.39) form an adjunction in $\mathrm{ENH}_{\mathrm{k}}$, i. e. the two diagrams in $\mathrm{ENH}_{\mathrm{k}}$ in (1.9) commute.

Proof. The proof is similar to that of Proposition 6.27 and left to the reader.

7.5.3. Some other lifts.

Lemma 7.19. Let $A$ be a k-algebra and $\alpha: Y \rightarrow X$ a morphism of topological spaces. Let $E \in \mathrm{C}\left(X_{A}\right)$ and $F \in \mathrm{C}\left(Y_{A}\right)$. The 2-morphisms

$$
\begin{aligned}
\underline{\alpha}^{-1} \underline{E} & =\mathbf{i} \alpha^{-1} \mathbf{i} E \stackrel{[\sim]}{\longleftarrow} \mathbf{i} \alpha^{-1} E=\underline{\alpha^{-1} E}, \\
\underline{\alpha_{!} F} & =\mathbf{i} \alpha_{!} F \rightarrow \mathbf{i} \alpha ! \mathbf{i} F=\underline{\alpha} ! \underline{F}
\end{aligned}
$$

in $\widetilde{\mathrm{ENH}}_{\mathrm{k}}$ define 2-morphisms

$$
\begin{array}{rlr}
\underline{\alpha}^{-1} \underline{E} & \stackrel{\sim}{\rightarrow} \underline{\alpha}^{-1} E \\
\underline{\alpha_{1} F} & \rightarrow \underline{\alpha_{!}} \underline{F} & \text { (always a } 2 \text {-isomorphism), } \\
& \text { (2-isomorphism if } F \text { is } h \text {-inj.) }
\end{array}
$$

in $\mathrm{ENH}_{\mathrm{k}}$ that enhance the obvious 2-morphisms $\mathbf{L} \alpha^{-1}(E) \stackrel{\sim}{\rightarrow} \alpha^{-1} E$ and $\alpha_{!} F \rightarrow$ $\mathbf{R} \alpha_{!}(F)$.

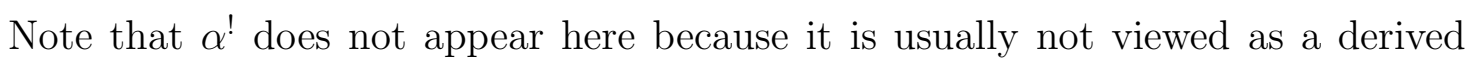
functor.

Proof. Left to the reader.

Lemma 7.20. Let $A$ be a k-algebra and $\alpha: Y \rightarrow X$ a morphism of topological spaces. Let $e: E \rightarrow E^{\prime}$ in $\mathrm{C}\left(X_{A}\right)$ and $f: F \rightarrow F^{\prime}$ in $\mathrm{C}\left(Y_{A}\right)$ be morphisms. Then the following diagrams in $\mathrm{ENH}_{\mathrm{k}}$ are commutative.

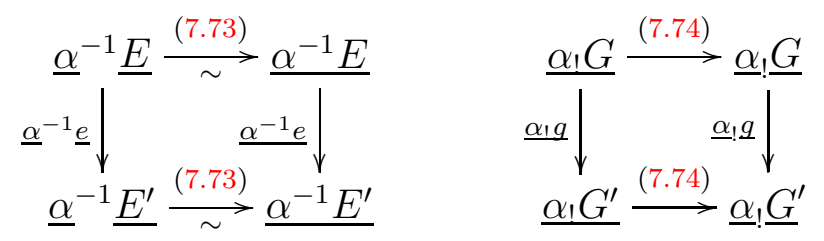

Proof. Left to the reader.

7.5.4. Subsequently constructed lifts of 2-(iso)morphisms. As in 6.5.4 we deduce some consequences. 
Lemma 7.21. Under the assumptions of Proposition 7.12 the composition

$$
\underline{\alpha}_{!} \underline{\beta}_{*}^{\prime} \stackrel{(7.15) \underline{\alpha}_{!} \underline{\beta}_{*}^{\prime}}{\longrightarrow} \underline{\beta}_{*} \underline{\beta}^{-1} \underline{\alpha}_{!} \underline{\beta}_{*}^{\prime} \stackrel{\underline{\beta}_{*}(7.43) \underline{\beta}_{*}^{\prime}}{\sim} \underline{\beta}_{*} \underline{\alpha}_{!}^{\prime} \underline{\beta}^{\prime-1} \underline{\beta}_{*}^{\prime} \stackrel{\underline{\beta}_{*} \underline{\alpha}_{!}^{\prime}(7.14)}{\longrightarrow} \underline{\beta}_{*} \underline{\alpha}_{!}^{\prime}
$$

in $\mathrm{ENH}_{\mathrm{k}}$ enhances the 2-morphism

$$
\mathbf{R} \alpha ! \mathbf{R} \beta_{*}^{\prime} \rightarrow \mathbf{R} \beta_{*} \mathbf{R} \alpha_{!}^{\prime}
$$

which is adjoint to the composition $\mathbf{L} \beta^{-1} \mathbf{R} \alpha_{!} \mathbf{R} \beta_{*}^{\prime} \stackrel{(7.44) \mathbf{R} \beta_{*}^{\prime}}{\sim} \mathbf{R} \alpha_{!}^{\prime} \mathbf{L} \beta^{\prime-1} \mathbf{R} \beta_{*}^{\prime} \rightarrow \mathbf{R} \alpha_{!}^{\prime}$.

Proof. The composition $\underline{\beta}^{-1} \underline{\alpha}_{!} \underline{\beta}_{*}^{\prime} \stackrel{(7.43) \underline{\beta}_{*}^{\prime}}{\longrightarrow} \underline{\alpha}_{!}^{\prime} \underline{\beta}^{\prime-1} \underline{\beta}_{*}^{\prime} \stackrel{\underline{\alpha}_{!}^{\prime}(7.14)}{\longrightarrow} \underline{\alpha}_{!}^{\prime}$ enhances the last composition in the formulation of the lemma, by Lemma 7.4, Proposition 7.12 and Remark 6.16. This implies the lemma.

Proposition 7.22. Under the assumptions of Proposition 7.12 the composition

$$
\begin{aligned}
\underline{\beta}_{*}^{\prime} \underline{\alpha}^{\prime !} \stackrel{(7.40) \underline{\beta}_{*}^{\prime} \underline{\alpha}^{\prime !}}{\longrightarrow} \underline{\alpha}^{!} \underline{\alpha}_{!} \underline{\beta}_{*}^{\prime} \underline{\alpha}^{\prime !} \stackrel{\underline{\alpha}^{!}(7.15) \underline{\alpha}_{!} \underline{\beta}_{*}^{\prime} \underline{\alpha}^{\prime}}{\longrightarrow} \underline{\alpha}^{!} \underline{\beta}_{*} \underline{\beta}^{-1} \underline{\alpha}_{!} \underline{\beta}_{*}^{\prime} \underline{\alpha}^{\prime !} \\
\stackrel{\underline{\alpha}^{\prime} \underline{\beta}_{*}(7.43) \underline{\beta}_{*}^{\prime} \underline{\alpha}^{\prime}}{\longrightarrow} \underline{\alpha}^{!} \underline{\beta}_{*} \underline{\alpha}_{!}^{\prime} \underline{\beta}^{\prime-1} \underline{\beta}_{*}^{\prime} \underline{\alpha}^{\prime !} \stackrel{\underline{\alpha}^{\prime} \underline{\beta}_{*} \underline{\alpha}_{!}^{\prime}(7.14) \underline{\alpha}^{\prime}}{\longrightarrow} \underline{\alpha}^{!} \underline{\beta}_{*} \underline{\alpha}_{!}^{\prime} \underline{\alpha}^{\prime !} \stackrel{\underline{\alpha}^{\prime} \underline{\beta}_{*}(7.39)}{\longrightarrow} \underline{\alpha}^{!} \underline{\beta}_{*}
\end{aligned}
$$

in $\mathrm{ENH}_{\mathrm{k}}$ is a 2-isomorphism

$$
\underline{\beta}_{*}^{\prime} \underline{\alpha}^{\prime !} \stackrel{\sim}{\rightarrow} \underline{\alpha}^{!} \underline{\beta}_{*}
$$

that enhances the 2-isomorphism

$$
\left(\mathbf{R} \beta_{*}^{\prime}\right) \alpha^{\prime !} \stackrel{\sim}{\rightarrow} \alpha ! \mathbf{R} \beta_{*}
$$

from [SS16, Thm. 8.3.(c)].

Remark 7.23. Note that (7.79) is a 2-isomorphism even though, in general, not all 2-morphisms in (7.78) are objectwise homotopy equivalences.

Proof. We claim that the 2-isomorphism $\underline{\beta}^{-1} \underline{\alpha}_{!} \stackrel{\sim}{\rightarrow} \underline{\alpha}_{!}^{\prime} \underline{\beta}^{\prime-1}$ in (7.43) has (7.79) as its conjugate 2 -morphism, and hence (7.79) is a 2 -isomorphism. To see the claim, specialize Remarks 7.25 and 7.26 below to the case $L=L_{1} L_{2}=\underline{\beta}^{-1} \underline{\alpha}_{!}$and $L^{\prime}=$ $L_{1}^{\prime} L_{2}^{\prime}=\underline{\alpha}_{!}^{\prime} \underline{\beta}^{\prime-1}$ and use the adjunctions $\left(\underline{\alpha}_{!}, \underline{\alpha}^{!}\right),\left(\underline{\alpha}_{!}^{\prime}, \underline{\alpha}^{\prime}\right),\left(\underline{\beta}^{-1}, \underline{\beta}_{*}\right)$ and $\left(\underline{\beta}^{\prime-1}, \underline{\beta}_{*}^{\prime}\right)$ in $\mathrm{ENH}_{\mathrm{k}}$ (see Proposition 7.18 and Lemma 7.4).

The 2-isomorphism (7.80) is by definition the 2-morphism that is conjugate to the 2isomorphism $\mathbf{L} \beta^{-1} \mathbf{R} \alpha_{!} \stackrel{\sim}{\rightarrow} \mathbf{R} \alpha_{!}^{\prime} \mathbf{L} \beta^{\prime-1}$ from (7.44). By the above argument, now using $L=L_{1} L_{2}=\mathbf{L} \beta^{-1} \mathbf{R} \alpha_{!}$and $L^{\prime}=L_{1}^{\prime} L_{2}^{\prime}=\mathbf{R} \alpha_{!}^{\prime} \mathbf{L} \beta^{\prime-1}$, it is equal to the composition

$$
\begin{aligned}
&\left(\mathbf{R} \beta_{*}^{\prime}\right) \alpha^{\prime !} \stackrel{\eta_{2}\left(\mathbf{R} \beta_{*}^{\prime}\right) \alpha^{\prime !}}{\longrightarrow} \alpha^{!} \mathbf{R} \alpha_{!}\left(\mathbf{R} \beta_{*}^{\prime}\right) \alpha^{\prime !} \stackrel{\alpha^{\prime} \eta_{1} \mathbf{R} \alpha_{!}\left(\mathbf{R} \beta_{*}^{\prime}\right) \alpha^{\prime !}}{\longrightarrow} \alpha^{!} \mathbf{R} \beta_{*} \mathbf{L} \beta^{-1} \mathbf{R} \alpha_{!}\left(\mathbf{R} \beta_{*}^{\prime}\right) \alpha^{\prime !} \\
& \stackrel{\alpha^{\prime} \mathbf{R} \beta_{*}(7.44)\left(\mathbf{R} \beta_{*}^{\prime}\right) \alpha^{\prime !}}{\longrightarrow} \alpha ! \mathbf{R} \beta_{*} \mathbf{R} \alpha_{!}^{\prime} \mathbf{L} \beta^{\prime-1}\left(\mathbf{R} \beta_{*}^{\prime}\right) \alpha^{\prime !} \\
& \stackrel{\alpha^{\prime} \mathbf{R} \beta_{*} \mathbf{R} \alpha_{!}^{\prime} \theta_{2}^{\prime} \alpha^{\prime !}}{\longrightarrow} \alpha^{!} \mathbf{R} \beta_{*}\left(\mathbf{R} \alpha_{!}^{\prime}\right) \alpha^{\prime !} \stackrel{\alpha^{\prime} \mathbf{R} \beta_{*} \theta_{1}^{\prime}}{\longrightarrow} \alpha^{!} \mathbf{R} \beta_{*}
\end{aligned}
$$

where $\eta_{1}, \eta_{2}$ and $\theta_{1}^{\prime}, \theta_{2}^{\prime}$ denote units and counits of the obvious adjunctions. 
Therefore, Lemma 7.4, Propositions 7.11, 7.12, and Remark 6.16 imply that the composition in (7.78) enhances the 2-isomorphism (7.80).

Remark 7.24. Assume that $(L, R, \eta, \theta)$ and $\left(L^{\prime}, R^{\prime}, \eta^{\prime}, \theta^{\prime}\right)$ are adjunctions between functors $L, L^{\prime}: \mathcal{A} \rightarrow \mathcal{C}$ and $R, R^{\prime}: \mathcal{C} \rightarrow \mathcal{A}$, respectively. Then any transformation $\lambda: L \rightarrow L^{\prime}$ gives rise to a unique transformation $\rho: R^{\prime} \rightarrow R$ such that

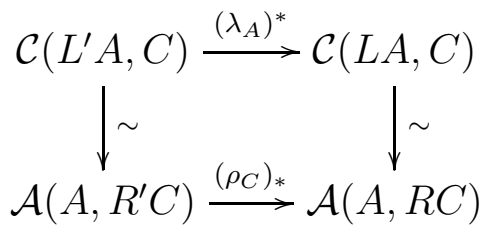

commutes for all $A \in \mathcal{A}$ and $C \in \mathcal{C}$, and conversely. Then $\rho$ and $\lambda$ are conjugate in the terminology of [ML98, IV.7], [Lip09, 3.3]. Moreover, $\lambda$ is an isotransformation if and only if $\rho$ is an isotransformation (cf. Remark 7.25 below). Similar statements hold for adjunctions between $\mathcal{K}$-functors. In fact, there is an abstract 2 -categorical statement explained in the following Remark 7.25.

Remark 7.25. This remark is a variant of Remark 7.24. Assume that $(L, R, \eta, \theta)$ and $\left(L^{\prime}, R^{\prime}, \eta^{\prime}, \theta^{\prime}\right)$ are adjunctions in a 2-category where $L$ and $L^{\prime}$ are 1-morphisms $\mathcal{A} \rightarrow \mathcal{C}$. Then any 2-morphism $\lambda: L \rightarrow L^{\prime}$ gives rise to a conjugate 2-morphism $\rho: R^{\prime} \rightarrow R$ defined as the composition

$$
R^{\prime} \stackrel{\eta R^{\prime}}{\longrightarrow} R L R^{\prime} \stackrel{R \lambda R^{\prime}}{\longrightarrow} R L^{\prime} R^{\prime} \stackrel{R \theta^{\prime}}{\longrightarrow} R
$$

which is illustrated by the following diagram.

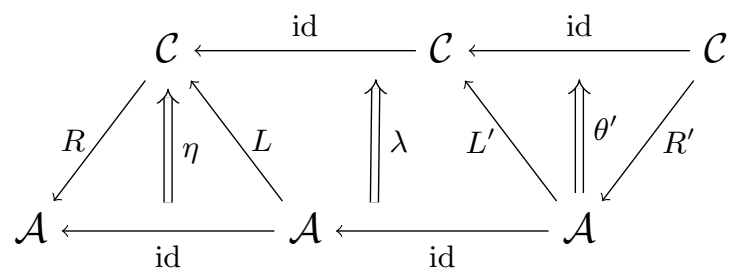

This construction defines a bijection between the set of 2-morphisms $L \rightarrow L^{\prime}$ and the set of 2-morphisms $R^{\prime} \rightarrow R$.

Let $\left(L^{\prime \prime}, R^{\prime \prime}, \eta^{\prime \prime}, \theta^{\prime \prime}\right)$ be another adjunction where $L^{\prime \prime}: \mathcal{A} \rightarrow \mathcal{C}$ is a 1 -morphism. Then any 2-morphism $\lambda^{\prime}: L^{\prime} \rightarrow L^{\prime \prime}$ similarly has a conjugate 2-morphism $\rho^{\prime}: R^{\prime \prime} \rightarrow R^{\prime}$. Pasting together the diagram (7.84) and the corresponding diagram defining $\rho^{\prime}$, the triangle identities easily imply that the 2-morphism conjugate to $\lambda^{\prime} \circ \lambda: L \rightarrow L^{\prime \prime}$ is $\rho \circ \rho^{\prime}$. In particular, $\lambda$ is a 2-isomorphism if and only if $\rho$ is a 2-isomorphism.

Remark 7.26. Assume that $\left(L_{1}, R_{1}, \eta_{1}, \theta_{1}\right)$ and $\left(L_{2}, R_{2}, \eta_{2}, \theta_{2}\right)$ are adjunctions between 1-morphisms $\mathcal{A} \underset{R_{2}}{\stackrel{L_{2}}{\rightleftarrows}} \mathcal{B} \underset{R_{1}}{\stackrel{L_{1}}{\rightleftarrows}} \mathcal{C}$ in a 2-category. Then $\left(L=L_{1} L_{2}, R=R_{2} R_{1}\right)$ 
together with

$$
\begin{aligned}
& \eta: \mathrm{id} \stackrel{\eta_{2}}{\longrightarrow} R_{2} L_{2} \stackrel{R_{2} \eta_{1} L_{2}}{\longrightarrow} R_{2} R_{1} L_{1} L_{2}, \\
& \theta: L_{1} L_{2} R_{2} R_{1} \stackrel{L_{1} \theta_{2} R_{1}}{\longrightarrow} L_{1} R_{1} \stackrel{\theta_{1}}{\longrightarrow} \text { id }
\end{aligned}
$$

as unit and counit form an adjunction.

Lemma 7.27. Let $A$ be a k-algebra and $\alpha: Y \rightarrow X$ a separated, locally proper morphism of topological spaces with $\alpha_{!}: \operatorname{Mod}\left(Y_{A}\right) \rightarrow \operatorname{Mod}\left(X_{A}\right)$ of finite cohomological dimension. Then the composition

$$
\begin{aligned}
& \underline{\operatorname{Hom}}\left(-, \underline{\alpha}^{!}(-)\right) \stackrel{(6.75)\left(\mathrm{id}, \underline{\alpha}^{!}\right)}{\sim} \Gamma \underline{\mathcal{H} o m}\left(-, \underline{\alpha}^{!}(-)\right) \\
& \stackrel{(6.64) \underline{\mathcal{H o m}}\left(-, \underline{\alpha}^{!}(-)\right)}{\sim} \underline{\Gamma} \underline{\alpha}_{*} \underline{\mathcal{H} o m}\left(-, \underline{\alpha}^{!}(-)\right) \stackrel{\Gamma(7.60)}{\sim} \underline{\Gamma} \underline{\mathcal{H} o m}\left(\underline{\alpha}_{!}(-),-\right) \\
& \sim \frac{(6.75)^{-1}\left(\underline{\alpha}_{!}(-), \mathrm{id}\right)}{\sim} \underline{\operatorname{Hom}}\left(\underline{\alpha}_{!}-,-\right)
\end{aligned}
$$

of 2-isomorphisms in $\mathrm{ENH}_{\mathbf{k}}$ enhances $\mathbf{R H o m}\left(-, \alpha^{!}(-)\right) \stackrel{\sim}{\rightarrow} \mathbf{R H o m}\left(\mathbf{R} \alpha_{!}(-),-\right)$.

The adjunction isomorphisms $\mathrm{D}_{Y_{A}}\left(G, \alpha^{!} E\right) \cong \mathrm{D}_{X_{A}}\left(\mathbf{R} \alpha_{!}(G), E\right)$ can be obtained as in Remark 6.34.

Proof. Use Remark 6.16, Lemmas 6.21, 6.23, and Proposition 7.16.

\section{The 2-Multicategory of FORMulas}

We provide a language which allows a compact formulation of almost all main results of this article, see Theorems 8.3 and 8.5 and Remark 8.4. All ringed sites, ringed spaces and algebras in this section are assumed to be $\mathcal{U}$-small; all algebras are commutative.

Let $\mathrm{k}$ be a field. Recall the category $\mathrm{fml}_{\mathrm{k}}$ from 7.2. Let $\widetilde{\mathrm{FML}}_{\mathrm{k}}$ be the free $\mathrm{k}$-linear 2-multicategory which has the same objects as $\mathrm{fml}_{\mathrm{k}}$, whose generating 1-morphisms are the generating morphisms of $\mathrm{fml}_{\mathrm{k}}$, and whose generating 2-morphisms are the 27 morphisms in the left column of table 1 on page 6 and the following generating 2-morphisms, and their opposites (cf. Remark 8.1 below).

(a) for each k-ringed site $(\mathcal{X}, \mathcal{O})$ and each morphism $g: G \rightarrow G^{\prime}$ in $\mathrm{C}(\mathcal{X})$ there is a 2-morphism $g: G \rightarrow G^{\prime}$;

(b) for each k-ringed site $(\mathcal{X}, \mathcal{O})$ and each pair $E, F \in \mathrm{C}(\mathcal{X})$ of objects there are

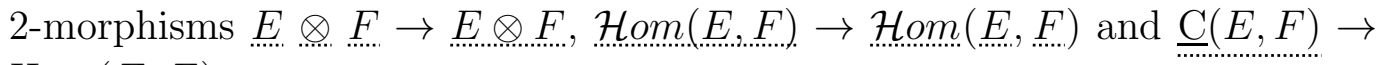
$\operatorname{Hom}(E, F)$

(c) for each morphism $\alpha:\left(\operatorname{Sh}(\mathcal{Y}), \mathcal{O}_{\mathcal{Y}}\right) \rightarrow\left(\operatorname{Sh}(\mathcal{X}), \mathcal{O}_{\mathcal{X}}\right)$ of k-ringed topoi and each object $E \in \mathrm{C}(\mathcal{X})$ (resp. $F \in \mathrm{C}(\mathcal{Y})$ ) there is a 2-morphism $\alpha^{*} E \rightarrow\left(\alpha^{*} E\right)$. $\left(\right.$ resp. $\left.\left(\alpha_{*} F\right) \rightarrow \alpha_{*} F\right)$ 
(d) for each k-algebra $A$ and each morphism $\alpha: Y \rightarrow X$ of topological spaces and each object $E \in \mathrm{C}\left(X_{A}\right)$ (resp. $F \in \mathrm{C}\left(Y_{A}\right)$ ) there is a 2-morphism $\alpha^{-1} E \rightarrow$ $\left(\alpha^{-1} E\right)$ (resp. $\left.\left(\alpha_{1} F\right) \rightarrow \alpha_{!} F\right)$

The k-linear 2-multicategory $\mathrm{FML}_{\mathrm{k}}$ is obtained from $\widetilde{\mathrm{FML}}_{\mathrm{k}}$ as follows. We require the following generating 2-morphisms and their opposites to be invertible: all 2morphisms labeled $\sim$ in the left column of table $1 ; g: G \rightarrow G^{\prime}$ if $g$ is a quasiisomorphism; $E \otimes F \rightarrow E \otimes F$ if $E$ or $F$ is h-flat; $\mathcal{H o m}(E, F) \rightarrow \mathcal{H o m}(E, F)$ and $\underline{\mathrm{C}}(E, F) \rightarrow \operatorname{Hom}(E, F)$ if $F$ is h-injective or if $E$ is h-flat and $F$ is weakly h-injective; $\alpha^{*} E \rightarrow\left(\alpha^{*} E\right)$ if $E$ is h-flat; $\left(\alpha_{*} F\right) \rightarrow \alpha_{*} F$ if $F$ is weakly h-injective; $\alpha^{-1} E \rightarrow\left(\alpha^{-1} E\right) ;\left(\alpha_{1} F\right) \rightarrow \alpha_{1} F$ if $F$ is h-injective. Moreover, we impose the relations $f . g=(f g)$. for all composable morphisms $f$ and $g$ in $\mathrm{C}(\mathcal{X})$, and $r g+r^{\prime} g^{\prime}=r g+r^{\prime} g^{\prime}$ for all morphisms $g, g^{\prime}: G \rightarrow G^{\prime}$ in $\mathrm{C}(\mathcal{X})$ and all scalars $r, r^{\prime} \in \mathrm{k}$.

These conditions imply that $\mathrm{id}_{G}=\mathrm{id}_{G}$ in $\mathrm{FML}_{\mathrm{k}}$, for $G \in \mathrm{C}(\mathcal{X})$, and that two morphisms $g$ and $g^{\prime}$ in $\mathrm{C}(\mathcal{X})$ which become equal in $\mathrm{D}(\mathcal{X})$ give rise to the same 2-morphism $g=g^{\prime}$ in $\mathrm{FML}_{\mathrm{k}}$.

Remark 8.1. The involution (-) op on $\mathrm{fml}_{\mathrm{k}}$ from 7.2 extends to $\widetilde{\mathrm{FML}}_{\mathrm{k}}$ and $\mathrm{FML}_{\mathrm{k}}$ in the obvious way by changing the direction of the 2-morphisms. For example, the 2-morphism $\tau: \alpha^{*} \alpha_{*} \rightarrow$ id: $\mathcal{Y}_{3} \rightarrow \mathcal{Y}$ is mapped to the 2-morphism $\tau^{\text {op }}:$ id $\rightarrow$ $\left(\alpha^{*}\right)^{\mathrm{op}}\left(\alpha_{*}\right)^{\mathrm{op}}: \mathcal{Y}^{\mathrm{op}} \rightarrow \mathcal{Y}^{\mathrm{op}}$. More formally, the involution is a functor $(-)^{\mathrm{op}}: \mathrm{FML}_{\mathrm{k}} \rightarrow$ $\mathrm{FML}_{\mathrm{k}}^{\mathrm{co}}$ where $\mathrm{FML}_{\mathrm{k}}^{\mathrm{co}}$ is obtained from $\mathrm{FML}_{\mathrm{k}}$ by reversing the directions of the 2morphisms.

There is a canonical functor $\widetilde{F M L}_{k} \rightarrow \mathrm{FML}_{\mathrm{k}}$ between k-linear 2-multicategories. The underlying multicategories of $\widehat{F M L}_{k}$ and $\mathrm{FML}_{\mathrm{k}}$ are both fml $\mathrm{f}_{\mathrm{k}}$. We extend our convention from Remark 5.14 to functors with source $\widetilde{F M L}_{k}$ or $\mathrm{FML}_{\mathrm{k}}$ in the obvious way.

Lemma 8.2. There is a unique functor

$$
\mathrm{D}: \mathrm{FML}_{\mathrm{k}} \rightarrow \mathrm{TRCAT}_{\mathrm{k}}
$$

of $\mathrm{k}$-linear 2-multicategories whose composition with $\widetilde{\mathrm{FML}}_{\mathrm{k}} \rightarrow \mathrm{FML}_{\mathrm{k}}$ extends the interpretation functor $\mathrm{D}: \mathrm{fml}_{\mathrm{k}} \rightarrow$ trcat $_{\mathrm{k}}$ of multicategories from (7.1), sends the 27 generating 2-morphisms in the left column of table 1 to the corresponding entries in the right column, and sends $g: G \rightarrow G^{\prime}$ to $g: G \rightarrow G^{\prime}, E \otimes F \rightarrow E \otimes F$ to $E \otimes \mathbf{L} F \rightarrow E \otimes F, \mathcal{H o m}(E, F) \rightarrow \mathcal{H o m}(E, F)$ to $\mathcal{H o m}(E, F) \rightarrow \mathbf{R} \mathcal{H o m}(E, F)$, $\underline{\mathrm{C}}(E, F) \rightarrow \underline{\operatorname{Hom}}(E, F)$ to $\underline{\mathrm{C}}(E, F) \rightarrow \mathbf{R H o m}(E, F), \alpha^{*} E \rightarrow\left(\alpha^{*} E\right)$ to $\mathbf{L} \alpha^{*}(E) \rightarrow$ $\alpha^{*} E,\left(\alpha_{*} F\right) \rightarrow \alpha_{*} F$ to $\alpha_{*} F \rightarrow \mathbf{R} \alpha_{*} F, \alpha^{-1} E \rightarrow\left(\alpha^{-1} E\right)$. to $\mathbf{L} \alpha^{-1}(E) \rightarrow \alpha^{-1} E$, and $\left(\alpha_{!} F\right) \rightarrow \alpha_{!} F$ to $\alpha_{!} F \rightarrow \mathbf{R} \alpha_{!} F$.

Proof. This is obvious. 
The main results of sections 6 and 7 can now be summarized in the following two theorems.

Theorem 8.3. Let $\mathrm{k}$ be a field. There is a unique functor

$$
\underline{I}: \mathrm{FML}_{\mathrm{k}} \rightarrow \mathrm{ENH}_{\mathrm{k}}
$$

of k-linear 2-multicategories whose composition with $\widetilde{\mathrm{FML}}_{\mathrm{k}} \rightarrow \mathrm{FML}_{\mathrm{k}}$ extends the interpretation functor $\mathbb{I}: \mathrm{fml}_{\mathrm{k}} \rightarrow \mathrm{enh}_{\mathrm{k}}$ of multicategories from (7.12), sends the 27 generating 2-morphisms in the left column of table 1 to the corresponding entries in the middle column, and sends $g: G \rightarrow G^{\prime}$ to $q: \underline{G} \rightarrow \underline{G}^{\prime}, \underline{E} \otimes \underline{F} \rightarrow \underline{E} \otimes F$ to $\underline{E} \underline{\otimes} \underline{F} \rightarrow \underline{E \otimes F}, \underline{\mathcal{H} o m}(E, F) \rightarrow \mathcal{H o m}(E, F)$ to $\underline{\mathcal{H} o m}(E, F) \rightarrow \underline{\mathcal{H} o m}(\underline{E}, \underline{F})$, $\underline{\mathrm{C}}(E, F) \rightarrow \underline{\operatorname{Hom}}(\underline{E}, \underline{F})$ to $\underline{\mathrm{C}}(E, F) \rightarrow \underline{\operatorname{Hom}}(\underline{E}, \underline{F}), \alpha^{*} \underline{E} \rightarrow\left(\alpha^{*} \underline{E}\right)$ to $\underline{\alpha}^{*} \underline{E} \rightarrow \underline{\left(\alpha^{*} E\right)}$, $\left(\alpha_{*} F\right) \rightarrow \alpha_{*} F$ to $\left(\alpha_{*} F\right) \rightarrow \underline{\alpha_{*}} \underline{F}, \alpha^{-1} \underline{E} \rightarrow\left(\alpha^{-1} E\right)$ to $\underline{\alpha}^{-1} \underline{E} \rightarrow \underline{\left(\alpha^{-1} E\right)}$, and $\left(\alpha_{!} F\right) \rightarrow \alpha_{!} F$ to $\underline{\left(\alpha_{!} F\right)} \rightarrow \underline{\alpha_{!}} \underline{F}$.

Proof. The results in sections 6 and 7 provide the relevant images in $\mathrm{ENH}_{\mathrm{k}}$ for the generating 2-morphisms of $\widetilde{\mathrm{FML}}_{\mathrm{k}}$ and show that they satisfy the defining relations of $\mathrm{FML}_{\mathrm{k}}$.

Remark 8.4. If we view all diagrams in 1.7 (except diagram (1.2)) as diagrams in $\mathrm{FML}_{\mathrm{k}}$ by replacing underlines by underdots, the interpretation functor (8.2) maps these diagrams to commutative diagrams (namely to the commutative diagrams in 1.7), and similarly for the commutative diagrams in Lemmas 6.32, 7.20. This follows from the results in 6.5.2, 7.5.2 and Lemmas 6.32, 7.20. Hence, when defining $\mathrm{FML}_{\mathrm{k}}$, we could additionally impose the condition that all these diagrams are commutative. If doing so, we would in particular have adjunctions $\left(\alpha^{*}, \alpha_{*}\right)$ and $\left(\alpha_{1}, \alpha^{!}\right)$in $\mathrm{FML}_{\mathrm{k}}$. This would imply that the 2-morphism $\beta_{*}^{\prime} \alpha^{\prime !} \rightarrow \alpha^{!} \beta_{*}$ in the left column of row (T2.8) of table 2 would be invertible (as the other 2-morphisms in this row): in analogy to (7.78), it is defined as the composition

$$
\begin{aligned}
& \beta_{* *}^{\prime} \alpha^{! !} \rightarrow \alpha^{!} \alpha_{!} \beta_{*}^{\prime} \alpha^{\prime !} \rightarrow \alpha^{!} \beta_{*} \beta^{-1} \alpha_{!} \beta_{* *}^{\prime} \alpha^{\prime !} \\
& \stackrel{\alpha^{!} \beta_{*}(\mathrm{~T} 1.27) \underline{\beta}_{*}^{\prime} \alpha^{\prime !}}{\rightarrow} \alpha^{!} \beta_{*} \alpha_{!}^{\prime} \beta^{\prime}{ }^{\prime-1} \beta_{* *}^{\prime} \alpha^{\prime !} \rightarrow \alpha^{!} \beta_{*} \alpha_{!}^{\prime} \alpha^{\prime !} \rightarrow \alpha^{!} \beta_{*},
\end{aligned}
$$

hence it is conjugate to the 2-isomorphism $\beta^{-1} \alpha_{!} \stackrel{(\mathrm{T} 1.27)}{\sim} \alpha_{!}^{\prime} \beta^{\prime-1}$ in $\mathrm{FML}_{\mathrm{k}}$ and invertible, by Remarks 7.25 and 7.26.

Theorem 8.5. Let $\mathrm{k}$ be a field. The pseudo-natural transformation (6.33) defines in fact a pseudo-natural transformation

$$
(\overline{[\mathbf{i}]}, \omega): \mathrm{D} \rightarrow[\underline{\mathbb{I}}]=[-] \circ \underline{\mathbb{I}}
$$

between functors $\mathrm{FML}_{\mathrm{k}} \rightarrow \mathrm{TRCAT}_{\mathrm{k}}$ of $\mathrm{k}$-linear 2-multicategories where $\mathrm{D}$ is (8.1) and [I] is the composition $\mathrm{FML}_{\mathrm{k}} \underset{\mathbb{I}}{\stackrel{(8.2)}{\longrightarrow}} \mathrm{ENH}_{\mathrm{k}} \underset{[-]}{\stackrel{(5.13)}{\longrightarrow}} \operatorname{TRCAT}_{\mathrm{k}}$ (see (2.7) for a diagram). 
Proof. The data required to define a pseudo-natural transformation $\mathrm{D} \rightarrow[\mathbb{I}]$ where $\mathrm{D}$ and $[\mathrm{I}]$ are considered as functors $\mathrm{fml}_{\mathrm{k}} \rightarrow$ trcat $_{\mathrm{k}}$ of multicategories or as functors $\mathrm{FML}_{\mathrm{k}} \rightarrow$ TRCAT $_{\mathrm{k}}$ of k-linear 2-multicategories coincide, by [Bor94, Def. 7.5.2]: the required natural transformation consists of a collection of morphisms indexed by the set $\mathrm{fml}_{\mathrm{k}}\left(S_{1}, \ldots, S_{n} ; T\right)=\operatorname{Obj}\left(\mathrm{FML}_{\mathrm{k}}\left(S_{1}, \ldots, S_{n} ; T\right)\right)$, for each given collection $S_{1}, \ldots, S_{n}, T \in \operatorname{Obj}\left(\mathrm{fml}_{\mathrm{k}}\right)=\operatorname{Obj}\left(\mathrm{FML}_{\mathrm{k}}\right)$ of objects. However, in the case of functors of k-linear 2-multicategories more conditions are imposed: the collection of morphisms is required to be compatible with morphisms in $\mathrm{FML}_{\mathrm{k}}\left(S_{1}, \ldots, S_{n} ; T\right)$. For the generating 2-morphisms of $\widetilde{F M L}_{\mathrm{k}}$ this compatibility boils down to our main results of sections 6 and 7, using Definition 6.15, for example to the fact that our 2-morphism $\underline{\alpha}^{*} \underline{\alpha}_{*} \rightarrow$ id $\left(\alpha^{*} \alpha_{*}\right.$, id)-enhances the 2-morphism $\mathbf{L} \alpha^{*} \mathbf{R} \alpha_{*} \rightarrow$ id. The theorem follows.

\section{SOME REMARKS ON 2-MORPHISMS IN ENH}

Recall the functor $\delta: \widetilde{\mathrm{ENH}}_{\mathrm{k}} \rightarrow \mathrm{ENH}_{\mathrm{k}}$ from (5.12). There are three statements about 2-morphisms in $\mathrm{ENH}_{\mathrm{k}}$ we would like to be true but do not know how to prove in general:

(I) A 2-morphism $\tau$ in $\widetilde{\mathrm{ENH}}_{\mathrm{k}}$ is an objectwise homotopy equivalence if and only if $\delta(\tau)$ is a 2-isomorphism in $\mathrm{ENH}_{\mathrm{k}}$.

(II) Any 2-morphism $\tau^{\prime}$ in $\mathrm{ENH}_{\mathrm{k}}$ can be represented by a roof, i. e. it has the form $\tau^{\prime}=\delta(v) \circ \delta(u)^{-1}$ where $u$ and $v$ are 2-morphisms in $\widetilde{\mathrm{ENH}}_{\mathrm{k}}$ and $u$ is an objectwise homotopy equivalence; moreover, for any such representation, $\tau^{\prime}$ is a 2-isomorphism if and only if $v$ is an objectwise homotopy equivalence.

(III) The functor [-]: $\mathrm{ENH}_{\mathrm{k}} \rightarrow \mathrm{TRCAT}_{\mathrm{k}}$ reflects 2-isomorphisms (i. e. it is conservative).

The aim of this section is to prove these statements under suitable assumptions on the sources and target of the involved 1-morphisms. The target must be a suitable category of sheaves or modules that we can control with model categorical techniques. Then either the sources must be sufficiently small (see Proposition 9.5) or the target must be made sufficiently large (see Proposition 9.9). We expect these results to be useful in applications. Our approach is based on the existence of certain model structures on functor categories which only seem to be available if the source category is small compared to the target category.

\subsection{Localizations of functor categories.}

Proposition 9.1. Let $\underline{\mathcal{N}}$ be a combinatorial $\mathcal{K}$-enriched model category (with respect to the universe $\mathcal{U})$. Assume that the localization functor $\lambda: \mathcal{M} \rightarrow \operatorname{Ho}(\mathcal{M})$ factors through the canonical functor $\mathcal{M} \rightarrow[\underline{\mathcal{N}}]$ as

$$
\mathcal{M} \rightarrow[\underline{\mathcal{N}}] \stackrel{\bar{\lambda}}{\rightarrow} \operatorname{Ho}(\mathcal{M})
$$


and that the restriction

$$
\left[\underline{\mathcal{M}}_{\mathrm{bifib}}\right] \rightarrow \mathrm{Ho}(\mathcal{M})
$$

of $\bar{\lambda}$ is an equivalence, where $\underline{\mathcal{M}}_{\mathrm{bifib}}$ is the full $\mathcal{K}$-category of $\underline{\mathcal{M}}$ of bifibrant (= fibrant and cofibrant) objects. Let $\underline{\mathcal{B}}$ be a full $\mathcal{K}$-subcategory of $\underline{\mathcal{M}}_{\text {bifib }}$ such that $[\underline{\mathcal{B}}] \subset\left[\underline{\mathcal{M}}_{\text {bifib }}\right]$ is a strictly full subcategory. Let $\underline{\mathcal{A}}$ be any $\mathcal{U}$-small $\mathcal{K}$-category. Let

$$
\delta: \operatorname{DGCAT}_{\mathrm{k}}(\underline{\mathcal{A}}, \underline{\mathcal{B}}) \rightarrow \mathrm{L}_{\mathrm{ohe}} \operatorname{DGCAT}_{\mathrm{k}}(\underline{\mathcal{A}}, \underline{\mathcal{B}})
$$

be the localization of $\operatorname{DGCAT}_{\mathrm{k}}(\underline{\mathcal{A}}, \underline{\mathcal{B}})$ with respect to the set of objectwise homotopy equivalences. Then

(a) we may and will assume that the category $\mathrm{L}_{\text {ohe }} \operatorname{DGCAT}_{\mathrm{k}}(\underline{\mathcal{A}}, \underline{\mathcal{B}})$ has $\mathcal{U}$-small Hom-sets, that it has the same set of objects as $\operatorname{DGCAT}_{\mathrm{k}}(\underline{\mathcal{A}}, \underline{\mathcal{B}})$ and that $\delta$ is the identity on objects;

(b) a morphism $\tau$ in $\operatorname{DGCAT}_{\mathrm{k}}(\underline{\mathcal{A}}, \underline{\mathcal{B}})$ is an objectwise homotopy equivalence if and only if $\delta(\tau)$ is an isomorphism;

(c) any morphism $\tau^{\prime}$ in $\mathrm{L}_{\text {ohe }} \operatorname{DGCAT}_{\mathrm{k}}(\underline{\mathcal{A}}, \underline{\mathcal{B}})$ has the form $\tau^{\prime}=\delta(v) \circ \delta(u)^{-1}$ where $u$ is an objectwise homotopy equivalence in $\operatorname{DGCAT}_{\mathrm{k}}(\underline{\mathcal{A}}, \underline{\mathcal{B}})$ and $v$ is a morphism in $\operatorname{DGCAT}_{\mathrm{k}}(\underline{\mathcal{A}}, \underline{\mathcal{B}})$; moreover, in any such representation, $\tau^{\prime}$ is an isomorphism if and only if $v$ is an objectwise homotopy equivalence;

(d) the unique functor $\overline{[-]}: \mathrm{L}_{\text {ohe }} \operatorname{DGCAT}_{\mathrm{k}}(\underline{\mathcal{A}}, \underline{\mathcal{B}}) \rightarrow \operatorname{CAT}_{\mathrm{k}}([\underline{\mathcal{A}}],[\underline{\mathcal{B}}])$ such that $\overline{[-]} \circ$ $\delta=[-]$ reflects isomorphisms.

Proof. Recall that $\mathcal{K}$ is an excellent model category, by Proposition 4.29.(a). Since $\underline{\mathcal{A}}$ is $\mathcal{U}$-small, we may consider $\operatorname{DGCAT}_{\mathrm{k}}(\underline{\mathcal{A}}, \underline{\mathcal{N}})$ as a model category equipped with the projective model structure given by [Lur09, Prop. A.3.3.2]. By definition, a morphism $\tau: F \rightarrow G$ in $\operatorname{DGCAT}_{\mathrm{k}}(\underline{\mathcal{A}}, \underline{\mathcal{M}})$ is a weak equivalence (resp. a fibration) if and only if $\tau_{A}: F(A) \rightarrow G(A)$ is a weak equivalence (resp. a fibration) for all $A \in \underline{\mathcal{A}}$.

We view $\operatorname{DGCAT}_{\mathrm{k}}(\underline{\mathcal{A}}, \underline{\mathcal{B}})$ as the full subcategory of $\operatorname{DGCAT}_{\mathrm{k}}(\underline{\mathcal{A}}, \underline{\mathcal{M}})$ consisting of $\mathcal{K}$-functors $\underline{\mathcal{A}} \rightarrow \underline{\mathcal{N}}$ that factor as $\underline{\mathcal{A}} \rightarrow \underline{\mathcal{B}} \rightarrow \underline{\mathcal{N}}$. We want to apply Theorem C.3 to

$$
\mathcal{S}:=\operatorname{DGCAT}_{\mathrm{k}}(\underline{\mathcal{A}}, \underline{\mathcal{B}}) \subset \mathcal{N}:=\operatorname{DGCAT}_{\mathrm{k}}(\underline{\mathcal{A}}, \underline{\mathcal{M}})
$$

and $h$ the composition

$h: \mathcal{N}=\operatorname{DGCAT}_{\mathrm{k}}(\underline{\mathcal{A}}, \underline{\mathcal{M}}) \stackrel{[-]}{\longrightarrow} \operatorname{CAT}_{\mathrm{k}}([\underline{\mathcal{A}}],[\underline{\mathcal{N}}]) \rightarrow \operatorname{CAT}([\underline{\mathcal{A}}],[\underline{\mathcal{M}}]) \stackrel{\bar{\lambda}_{*}}{\rightarrow} \operatorname{CAT}([\underline{\mathcal{A}}], \operatorname{Ho}(\mathcal{M}))$

where CAT is the 2-category of categories. Note that $\tau$ as above is a weak equivalence if and only if $h(\tau)$ is an isomorphism, by [Hir03, Thm. 8.3.10].

We need to show that all objects of $\mathcal{S}$ admit fibrant cofibrant approximations and cofibrant fibrant approximations in $\mathcal{S}$. Note that the initial (resp. terminal) object of $\mathcal{N}$ is the $\mathcal{K}$-functor $\underline{\mathcal{A}} \rightarrow \underline{\mathcal{N}}$ mapping each object of $\underline{\mathcal{A}}$ to the initial (resp. terminal) object of $\mathcal{M}$. Therefore, all objects of $\mathcal{S}$ are fibrant by the definition of the projective 
model structure and our assumption $\underline{\mathcal{B}} \subset \underline{\mathcal{M}}_{\text {bifib }} \subset \underline{\mathcal{N}}_{\text {fib }}$; hence cofibrant fibrant approximations in $\mathcal{S}$ exist trivially for all objects of $\mathcal{S}$.

Let $E$ be an object of $\mathcal{S}$ and $\rho: G \rightarrow E$ a fibrant cofibrant approximation in $\mathcal{N}$. For $A \in \underline{\mathcal{A}}$ the morphism $\rho_{A}: G(A) \rightarrow E(A)$ is a trivial fibration by the definition of the projective model structure. Since $E(A)$ is fibrant so is $G(A)$. By [Toë07, Prop. 3.3], $G(A)$ is cofibrant because $G$ is cofibrant. The object $E(A)$ is bifibrant by the assumption $\underline{\mathcal{B}} \subset \underline{\mathcal{M}}_{\text {bifib }}$. So $\rho_{A}$ is a weak equivalence between bifibrant objects, and the equivalence (9.2) shows that $\rho_{A}$ is an isomorphism in $\left[\underline{\mathcal{M}}_{\text {bifib }}\right]$. Since $E(A) \in \underline{\mathcal{B}}$ and $[\underline{\mathcal{B}}] \subset\left[\underline{\mathcal{M}}_{\text {bifib }}\right]$ is strictly full we obtain $G(A) \in \underline{\mathcal{B}}$. Hence $G \in \mathcal{S}$. This shows that fibrant cofibrant approximations in $\mathcal{S}$ exist for all objects of $\mathcal{S}$.

Now Theorem C.3.(a) applies to the localization of $\mathcal{S}=\operatorname{DGCAT}_{\mathrm{k}}(\underline{\mathcal{A}}, \underline{\mathcal{B}})$ with respect to the set of weak equivalences.

We claim that a morphism in $\mathcal{S}$ is a weak equivalence if and only if it is an objectwise homotopy equivalence. The restriction of $h$ to $\mathcal{S}$ is equal to the composition

$$
\mathcal{S}=\operatorname{DGCAT}_{\mathrm{k}}(\underline{\mathcal{A}}, \underline{\mathcal{B}}) \stackrel{[-]}{\longrightarrow} \operatorname{CAT}_{\mathrm{k}}([\underline{\mathcal{A}}],[\underline{\mathcal{B}}]) \rightarrow \operatorname{CAT}([\underline{\mathcal{A}}],[\underline{\mathcal{B}}]) \rightarrow \operatorname{CAT}([\underline{\mathcal{A}}], \operatorname{Ho}(\mathcal{M}))
$$

whose third arrow is induced by the full and faithful composition $[\underline{\mathcal{B}}] \subset\left[\underline{\mathcal{M}}_{\text {bifib }}\right] \stackrel{\sim}{\rightarrow}$ $\operatorname{Ho}(\mathcal{M})$. Therefore this third arrow is full and faithful and in particular reflects isomorphisms. The second arrow obviously reflects isomorphisms. These statements imply the claim, proving (a).

Moreover, (b), (c), and (d) follow from the above facts and parts (c), (e), (f), and (g) of Theorem C.3.

Proposition 9.2. Let $\underline{\mathcal{N}}$ be either

(a) $\underline{\mathrm{C}}(\mathcal{X})$ with $\mathrm{C}(\mathcal{X})$ carrying the $\mathbb{I}$-model structure, for a $\mathcal{U}$-small $\mathrm{k}$-ringed site $(\mathcal{X}, \mathcal{O})$, or

(b) $\underline{\operatorname{Mod}}(\underline{\mathrm{e}})$ with $\operatorname{Mod}(\underline{\mathrm{e}})$ carrying the $\mathbb{I}$ - or $\mathbb{P}$-model structure, for a $\mathcal{U}$-small $\mathcal{K}$ category $\underline{\mathrm{e}}$.

Then $\underline{\mathcal{M}}$ satisfies the assumptions imposed on $\underline{\mathcal{M}}$ in Proposition 9.1.

Proof. By Theorem 4.30, $\underline{\mathcal{N}}$ is a combinatorial $\mathcal{K}$-enriched model category. Two morphisms in $\mathcal{M}$ that are homotopic in the sense that they are equal in $[\underline{\mathcal{N}}]$ are left (resp. right) homotopic in the model categorical sense (use the obvious cylinder (resp. path) object if $\mathcal{M}$ carries the $\mathbb{I}$ - (resp. $\mathbb{P}$-)model structure). Therefore, using [Hir03, Lemma 8.3.4], the localization functor $\lambda: \mathcal{M} \rightarrow \operatorname{Ho}(\mathcal{M})$ factors through the canonical functor $\mathcal{M} \rightarrow[\underline{\mathcal{M}}]$ as $\mathcal{M} \rightarrow[\underline{\mathcal{N}}] \stackrel{\bar{\lambda}}{\rightarrow} \operatorname{Ho}(\mathcal{M})$. Moreover, the induced functor $\left[\underline{\mathcal{M}}_{\text {bifib }}\right] \rightarrow \operatorname{Ho}(\mathcal{M})$ is an equivalence, by [Hir03, Thm. 8.3.6]. Here we use the fact that maps in $\mathcal{M}$ between bifibrant objects are homotopic in the model categorical sense if

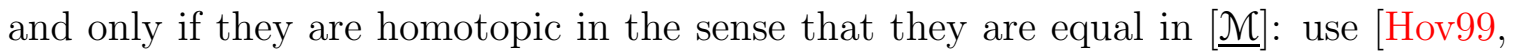
Cor. 1.2.6] and cylinder (resp. path) objects as above. 
Remark 9.3. We remind the reader that all objects of $\underline{\mathrm{C}}(\mathcal{X})$ are $\mathbb{I}$-cofibrant and that $\underline{\mathbb{I}}(\mathcal{X})=\underline{\mathrm{C}}(\mathcal{X})_{\mathbb{I} \text {-fib }}=\underline{\mathrm{C}}(\mathcal{X})_{\mathbb{I} \text {-bifib }}$, see 4.1.1 and 4.2.1. Similarly, $\underline{\mathbb{M} \text { od }}(\underline{\mathcal{C}})=$ $\underline{\operatorname{Mod}}(\underline{\mathrm{C}})_{\mathbb{I} \text {-cof }}$ and $\underline{\mathbb{M} \operatorname{Mod}}(\underline{\mathrm{C}})=\underline{\operatorname{Mod}}(\underline{\mathrm{C}})_{\mathbb{I} \text {-fib }}=\underline{\mathbb{M o d}}(\underline{\mathrm{C}})_{\mathbb{I} \text {-bifib }}$, and $\underline{\mathbb{M o d}}(\underline{\mathrm{C}})=\underline{\mathbb{M o d}}(\underline{\mathrm{e}})_{\mathbb{P} \text {-fib }}$ and $\underline{\mathbb{P M o d}}(\underline{\mathcal{C}})=\underline{\operatorname{Mod}}(\underline{\mathcal{C}})_{\mathbb{P} \text {-cof }}=\underline{\mathbb{M o d}}(\underline{\mathcal{C}})_{\mathbb{P} \text {-bifib }}$, see 4.3.1.

Remark 9.4. Consider $\underline{\mathrm{C}}(\mathcal{X})$ with $\mathrm{C}(\mathcal{X})$ carrying the $\mathbb{I}$-model structure, for a k-ringed site $(\mathcal{X}, \mathcal{O})$. The composition $\mathrm{C}(\mathcal{X}) \rightarrow[\underline{\mathrm{C}}(\mathcal{X})] \rightarrow \mathrm{D}(\mathcal{X})$ maps weak equivalences to isomorphisms and hence factors through the homotopy category $\mathrm{C}(\mathcal{X}) \rightarrow \operatorname{Ho}(\mathrm{C}(\mathcal{X}))$ to a functor $\operatorname{Ho}(\mathrm{C}(\mathcal{X})) \rightarrow \mathrm{D}(\mathcal{X})$. This functor is an isomorphism of categories. The subcategory of bifibrant objects of $\mathrm{C}(\mathcal{X})$ is $\mathbb{I}(\mathcal{X})$, and it is well-known that $[\mathbb{I}(\mathcal{X})] \rightarrow$ $\mathrm{D}(\mathcal{X})$ is an equivalence, cf. 4.1.2. This gives another point of view on the proof of part (a) of Proposition 9.2, and similarly for part (b) where $\operatorname{Ho}(\mathbb{M o d}(\underline{\mathcal{C}})) \stackrel{\sim}{\rightarrow} \mathrm{D}(\underline{\mathcal{C}})$ is an isomorphism.

9.2. Small sources. Let $\mathcal{U} \in \mathcal{V}$ be universes as in 5 .

Let $\underline{\mathcal{A}}_{1}, \ldots, \underline{\mathcal{A}}_{n}, \underline{\mathcal{B}}$ be objects of $\mathrm{ENH}_{\mathrm{k}}$. Recall that

$$
\delta: \widetilde{\mathrm{ENH}}_{\mathrm{k}}\left(\underline{\mathcal{A}}_{1}, \ldots, \underline{\mathcal{A}}_{n} ; \underline{\mathcal{B}}\right) \rightarrow \mathrm{ENH}_{\mathrm{k}}\left(\underline{\mathcal{A}}_{1}, \ldots, \underline{\mathcal{A}}_{n} ; \underline{\mathcal{B}}\right)
$$

denotes the additive k-localization with respect to the set of objectwise homotopy equivalences. We can now prove the statements (I), (II), (III) if the sources $\underline{\mathcal{A}}_{1}, \ldots, \underline{\mathcal{A}}_{n}$ are $\mathcal{U}$-small and the target $\underline{\mathcal{B}}$ is a suitable category of sheaves or modules.

Proposition 9.5. Let $\underline{\mathcal{A}}_{1}, \ldots, \underline{\mathcal{A}}_{n}$ be additive pretriangulated $\mathcal{U}$-small $\mathcal{K}$-categories. Let $\underline{\mathcal{N}}$ be either

- $\underline{I}(\mathcal{X})$, for a $\mathcal{U}$-small $\mathrm{k}$-ringed site $(\mathcal{X}, \mathcal{O})$, or

- $\underline{\mathbb{M M o d}}(\underline{\mathrm{C}})$ or $\underline{\mathbb{P M o d}}(\underline{\mathrm{C}})$, for a $\mathcal{U}$-small $\mathcal{K}$-category $\underline{\mathrm{C}}$.

Assume that $\underline{\mathcal{B}}$ is an additive pretriangulated full $\mathcal{K}$-subcategory of $\underline{\mathcal{N}}$ such that $[\underline{\mathcal{B}}]$ is a strictly full subcategory of [ㅅN]. Then the following statements hold true.

(a) A morphism $\tau$ in $\widetilde{\mathrm{ENH}}_{\mathrm{k}}\left(\underline{\mathcal{A}}_{1}, \ldots, \underline{\mathcal{A}}_{n} ; \underline{\mathcal{B}}\right)$ is an objectwise homotopy equivalence if and only if $\delta(\tau)$ is an isomorphism.

(b) Any morphism $\tau^{\prime}$ in $\operatorname{ENH}_{\mathrm{k}}\left(\underline{\mathcal{A}}_{1}, \ldots, \underline{\mathcal{A}}_{n} ; \underline{\mathcal{B}}\right)$ has the form $\tau^{\prime}=\delta(v) \circ \delta(u)^{-1}$ where $u$ and $v$ are morphisms in $\widetilde{\operatorname{ENH}}_{\mathrm{k}}\left(\underline{\mathcal{A}}_{1}, \ldots, \underline{\mathcal{A}}_{n} ; \underline{\mathcal{B}}\right)$ and $u$ is an objectwise homotopy equivalence; moreover, for any such representation, $\tau^{\prime}$ is a 2-isomorphism if and only if $v$ is an objectwise homotopy equivalence.

(c) The functor $[-]: \operatorname{ENH}_{\mathrm{k}}\left(\underline{\mathcal{A}}_{1}, \ldots, \underline{\mathcal{A}}_{n} ; \underline{\mathcal{B}}\right) \rightarrow \operatorname{TRCAT}_{\mathrm{k}}\left(\left[\underline{\mathcal{A}}_{1}\right], \ldots,\left[\underline{\mathcal{A}}_{n}\right] ;[\underline{\mathcal{B}}]\right)$ reflects isomorphisms.

Remark 9.6. This remark provides examples of possible source categories $\underline{\mathcal{A}}_{1}, \ldots, \underline{\mathcal{A}}_{n}$ in Proposition 9.5. Let $(\mathcal{X}, \mathcal{O})$ be a $\mathcal{U}$-small k-ringed site. Then $\mathbb{I}(\mathcal{X})$ is an object of $\mathrm{ENH}_{\mathrm{k}}$ which has $\mathcal{U}$-small Hom-sets and objects in $\mathcal{U}$, as explained in Remark 5.7. In general, however, $\mathbb{I}(\mathcal{X})$ is not $\mathcal{U}$-small. But there are many additive pretriangulated 
$\mathcal{U}$-small full $\mathcal{K}$-subcategories $\underline{\mathcal{A}}$ of $\underline{\mathbb{I}}(\mathcal{X})$ (that may enhance some interesting subcategories of $\mathrm{D}(\mathcal{X})$ defined by finiteness conditions). For example, take any strongly pretriangulated full $\mathcal{K}$-subcategory of $\mathbb{I}(\mathcal{X})$ whose set of objects is $\mathcal{U}$-small. One may produce such a subcategory iteratively by starting with any $\mathcal{U}$-small set of objects of $\underline{\mathbb{I}}(\mathcal{X})$.

Proof. If $\underline{\mathcal{N}}$ is $\underline{\mathbb{I}}(\mathcal{X})$, let $\underline{\mathcal{N}}=\underline{\mathrm{C}}(\mathcal{X})$ with $\mathcal{M}$ carrying the $\mathbb{I}$-model structure. If $\underline{\mathcal{N}}$ is $\underline{\mathbb{M} \operatorname{Mod}}(\underline{\mathcal{C}})$ (resp. $\underline{\mathbb{P M o d}}(\underline{\mathcal{C}})$ ), let $\underline{\mathcal{M}}=\underline{\mathbb{M o d}}(\underline{\mathcal{C}})$ with $\mathcal{M}$ carrying the $\mathbb{I}$-model structure (resp. the $\mathbb{P}$-model structure). By Proposition 9.2 and the fact that $\underline{\mathcal{B}} \subset \underline{\mathcal{N}}=\underline{\mathcal{M}}_{\text {bifib }}$ (see Remark 9.3) we can apply Proposition 9.1 (using the universe $\mathcal{U}$ and the $\mathcal{U}$-small $\mathcal{K}$-category $\left.\underline{\mathcal{A}}_{1} \otimes \cdots \otimes \underline{\mathcal{A}}_{n}\right)$. All claims follow if we remember that the underlying functor of the additive k-localization is the ordinary localization, see Proposition 5.5.

Remark 9.7. The assumption in Proposition 9.5 that the $\mathcal{K}$-categories $\underline{\mathcal{A}}_{1}, \ldots, \underline{\mathcal{A}}_{n}$ are $\mathcal{U}$-small is important for the proof. The assumption that they are additive pretriangulated was just imposed to ensure that they are objects of $\mathrm{ENH}_{\mathrm{k}}$. If we omit it, Proposition 9.5 remains true if we replace $\widetilde{\operatorname{ENH}}_{\mathrm{k}}\left(\underline{\mathcal{A}}_{1}, \ldots, \underline{\mathcal{A}}_{n} ; \underline{\mathcal{B}}\right) \rightarrow \operatorname{ENH}_{\mathrm{k}}\left(\underline{\mathcal{A}}_{1}, \ldots, \underline{\mathcal{A}}_{n} ; \underline{\mathcal{B}}\right)$ by $\operatorname{DGCAT}_{\mathrm{k}}\left(\underline{\mathcal{A}}_{1}, \ldots, \underline{\mathcal{A}}_{n} ; \underline{\mathcal{B}}\right) \rightarrow \mathrm{L}_{\mathrm{ohe}} \operatorname{DGCAT}_{\mathrm{k}}\left(\underline{\mathcal{A}}_{1}, \ldots, \underline{\mathcal{A}}_{n} ; \underline{\mathcal{B}}\right)$ and $\mathrm{TRCAT}_{\mathrm{k}}$ by $\mathrm{CAT}_{\mathrm{k}}$.

Example 9.8. Let

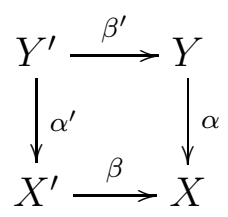

be a cartesian diagram of $\mathcal{U}$-small k-schemes. Then there is a base change 2 -morphism

$$
\mathbf{L} \beta^{*} \mathbf{R} \alpha_{*} \rightarrow \mathbf{R} \alpha_{*}^{\prime} \mathbf{L} \beta^{*}: \mathrm{D}(Y) \rightarrow \mathrm{D}\left(X^{\prime}\right)
$$

in TRCAT $_{k}$ constructed in the usual way using adjunction units and counits, see e. g. [Lip09, Prop. 3.7.2]. This construction has an obvious analog in $\mathrm{ENH}_{\mathrm{k}}$ providing a 2-morphism

$$
\underline{\beta}^{*} \underline{\alpha}_{*} \rightarrow \underline{\alpha}_{*}^{\prime} \underline{\beta}^{\prime *}: \underline{\mathbb{I}}(Y) \rightarrow \underline{\mathbb{I}}\left(X^{\prime}\right)
$$

in $\mathrm{ENH}_{\mathrm{k}}$ that enhances (9.9). Let $\mathrm{D}_{\mathrm{qc}}(Y)$ (resp. $\mathbb{I}_{\mathrm{qc}}(Y)$ ) be the subcategory of $\mathrm{D}(Y)$ (resp. $\mathbb{I}(Y))$ of objects with quasi-coherent cohomology. We assume that the 2morphism (9.9) restricts to a 2-isomorphism

$$
\mathbf{L} \beta^{*} \mathbf{R} \alpha_{*} \stackrel{\sim}{\rightarrow} \mathbf{R} \alpha_{*}^{\prime} \mathbf{L} \beta^{*}: \mathrm{D}_{\mathrm{qc}}(Y) \rightarrow \mathrm{D}\left(X^{\prime}\right)
$$

(see [Lip09, Prop. 3.9.5 or Thm. 3.10.3] for sufficient conditions). Let

$$
\tau^{\prime}: \underline{\beta}^{*} \underline{\alpha}_{*} \rightarrow \underline{\alpha}_{*}^{\prime} \underline{\beta}^{*}: \underline{\mathbb{I}}_{\mathrm{qc}}(Y) \rightarrow \underline{\mathbb{I}}\left(X^{\prime}\right)
$$


be the corresponding restriction of (9.10). It is a 2-morphism in $\mathrm{ENH}_{\mathrm{k}}$ but we do not know whether it is invertible. The fact that (9.11) is a 2-isomorphism implies (use Definition (6.15)) that

$$
\left[\tau^{\prime}\right]:\left[\underline{\beta}^{*}\right]\left[\underline{\alpha}_{*}\right] \rightarrow\left[\underline{\alpha}_{*}^{\prime}\right]\left[\underline{\beta}^{\prime *}\right]:\left[\underline{\mathbb{I}}_{\mathrm{qc}}(Y)\right] \rightarrow\left[\underline{\mathbb{I}}\left(X^{\prime}\right)\right]
$$

is a 2 -isomorphism. Let $\underline{\mathcal{A}}$ be a strongly pretriangulated $\mathcal{U}$-small full $\mathcal{K}$-subcategory of $\underline{\mathbb{I}}_{\mathrm{qc}}(Y)$, cf. Remark 9.6. Then the restriction

$$
\tau^{\prime} \underline{\mathcal{A}}_{\underline{\mathcal{A}}}: \underline{\beta}^{*} \underline{\alpha}_{*} \rightarrow \underline{\alpha}_{*}^{\prime} \underline{\beta}^{* *}: \underline{\mathcal{A}} \rightarrow \underline{\mathbb{I}}\left(X^{\prime}\right)
$$

of (9.12) to $\underline{\mathcal{A}}$ is an isomorphism in $\operatorname{ENH}_{\mathrm{k}}\left(\underline{\mathcal{A}}, \underline{\mathbb{I}}\left(X^{\prime}\right)\right)$, by Proposition 9.5.(c). Moreover, by part (b), this restriction is represented by a roof

$$
\underline{\beta}^{*} \underline{\alpha}_{*} \stackrel{u}{\leftarrow} F \stackrel{v}{\rightarrow} \underline{\alpha}_{*}^{\prime} \underline{\beta}^{*}
$$

of objectwise homotopy equivalences in $\operatorname{DGCAT}_{\mathrm{k}}\left(\underline{\mathcal{A}}, \underline{\mathbb{I}}\left(X^{\prime}\right)\right)$ where $F: \underline{\mathcal{A}} \rightarrow \underline{\mathbb{I}}\left(X^{\prime}\right)$ is some $\mathcal{K}$-functor.

9.3. Large targets. Let $\mathcal{U} \in \mathcal{V}$ be universes as in 5 . In this subsection we need two additional universes $\mathcal{U}^{\prime} \in \mathcal{V}^{\prime}$ with $\mathcal{V} \subset \mathcal{U}^{\prime}$.

Assume that $\underline{\mathcal{B}}$ is an additive pretriangulated full $\mathcal{K}$-subcategory of $\underline{\mathbb{I}}(\mathcal{X})=\underline{\mathbb{I}}(\mathcal{X} ; \mathcal{U})$ where $(\mathcal{X}, \mathcal{O})$ is a $\mathcal{U}$-small k-ringed site; we have included $\mathcal{U}$ in the notation to emphasize that we consider sheaves of $\mathcal{U}$-small modules.

By Proposition B.2 we know that we can view $\underline{\mathbb{I}}(\mathcal{X} ; \mathcal{U})$ as a full $\mathcal{K}_{\mathcal{U}^{\prime} \text {-subcategory of }}$

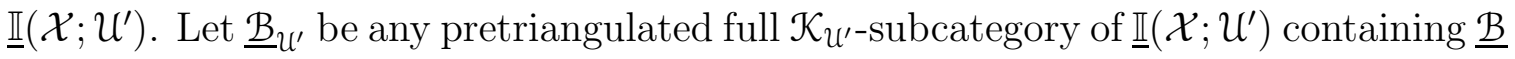
whose homotopy category is closed under isomorphisms in $\left[\mathbb{I}\left(\mathcal{X} ; \mathcal{U}^{\prime}\right)\right]$ (possible choices are $\mathbb{I}\left(\mathcal{X} ; \mathcal{U}^{\prime}\right)$ itself, or the smallest such subcategory: its set of objects is the set of objects of the essential image of $[\underline{\mathcal{B}}]$ in $\left.\left[\underline{\mathbb{I}}\left(\mathcal{X} ; \mathcal{U}^{\prime}\right)\right]\right)$. It is automatically strongly pretriangulated and in particular additive.

Remember that all objects of $\widetilde{\mathrm{ENH}}_{\mathrm{k}}$ and $\mathrm{ENH}_{\mathrm{k}}$ are $\mathcal{V}$-small $\mathcal{K}$-categories. Define $\widetilde{\mathrm{ENH}_{\mathrm{k}}} \mathcal{V}^{\prime}$ and $\mathrm{ENH}_{\mathrm{k}}^{\mathcal{V}^{\prime}}$ in the obvious way by taking as objects all additive pretriangulated $\mathcal{V}^{\prime}$-small $\mathcal{K}$-categories. There are obvious change of universe functors $\widetilde{\mathrm{ENH}}_{\mathrm{k}} \rightarrow \widetilde{\mathrm{ENH}}_{\mathrm{k}}^{\mathcal{V}^{\prime}}$ and $\mathrm{ENH}_{\mathrm{k}} \rightarrow \mathrm{ENH}_{\mathrm{k}}^{\mathcal{V}^{\prime}}$. Define $\mathrm{DGCAT}_{\mathrm{k}}^{\mathcal{V}^{\prime}}$ and $\mathrm{TRCAT}_{\mathrm{k}}^{\mathcal{V}^{\prime}}$ similarly by allowing $\mathcal{V}^{\prime}$-small categories as objects.

Let $\underline{\mathcal{A}}_{1}, \ldots, \underline{\mathcal{A}}_{n}$ be objects of $\mathrm{ENH}_{\mathrm{k}}$. Composition with $\underline{\mathcal{B}} \rightarrow \underline{\mathcal{B}}_{u^{\prime}}$ yields the horizontal functors in the following commutative diagram of $\mathrm{k}$-linear categories.

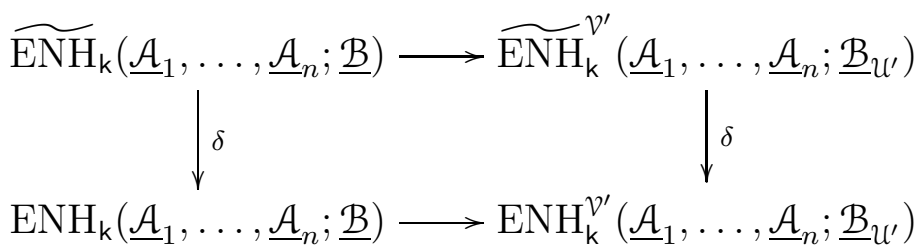

The advantage of the right column in this diagram is that the $\mathcal{V}$-small categories $\underline{\mathcal{A}}_{i}$ are in particular $\mathcal{U}^{\prime}$-small and that $\mathcal{B}_{\mathcal{U}^{\prime}}$ lives in the model category $\mathrm{C}\left(\mathcal{X} ; \mathcal{U}^{\prime}\right)$ which is 
a model category with respect to the universe $\mathcal{U}^{\prime}$. In this situation we can prove the following version of statements (I), (II), (III).

Proposition 9.9. Under the above assumptions, the following statements hold true.

(a) A morphism $\tau$ in $\widetilde{\operatorname{ENH}}_{\mathrm{k}}^{\nu^{\prime}}\left(\underline{\mathcal{A}}_{1}, \ldots, \underline{\mathcal{A}}_{n} ; \underline{\mathcal{B}}_{u^{\prime}}\right)$ is an objectwise homotopy equivalence if and only if $\delta(\tau)$ is an isomorphism.

(b) Any morphism $\tau^{\prime}$ in $\mathrm{ENH}_{\mathrm{k}}^{\mathcal{V}^{\prime}}\left(\underline{\mathcal{A}}_{1}, \ldots, \underline{\mathcal{A}}_{n} ; \underline{\mathcal{B}}_{u^{\prime}}\right)$ has the form $\tau^{\prime}=\delta(v) \circ \delta(u)^{-1}$ where $u$ and $v$ are morphisms in $\widetilde{\mathrm{ENH}_{\mathrm{k}}^{\nu^{\prime}}}\left(\underline{\mathcal{A}}_{1}, \ldots, \underline{\mathcal{A}}_{n} ; \underline{\mathcal{B}}_{u^{\prime}}\right)$ and $u$ is an objectwise homotopy equivalence; moreover, for any such representation, $\tau^{\prime}$ is a 2-isomorphism if and only if $v$ is an objectwise homotopy equivalence.

(c) The functor $[-]: \operatorname{ENH}_{\mathrm{k}}^{\mathcal{V}^{\prime}}\left(\underline{\mathcal{A}}_{1}, \ldots, \underline{\mathcal{A}}_{n} ; \underline{\mathcal{B}}_{u^{\prime}}\right) \rightarrow \operatorname{TRCAT}_{\mathrm{k}}^{\mathcal{V}^{\prime}}\left(\left[\underline{\mathcal{A}}_{1}\right], \ldots,\left[\underline{\mathcal{A}}_{n}\right] ;\left[\underline{\mathcal{B}}_{u^{\prime}}\right]\right)$ reflects isomorphisms.

Proof. This is a direct application of Proposition 9.5 if we work with the universes $\mathcal{U}^{\prime} \in \mathcal{V}^{\prime}$ instead of the universes $\mathcal{U} \in \mathcal{V}$.

Remark 9.10. If we assume that $\underline{B}$ is an additive pretriangulated full $\mathcal{K}$-subcategory of $\underline{\mathbb{M M o d}}(\underline{\mathcal{C}})$ or $\underline{\mathbb{P M o d}}(\underline{\mathcal{C}})$, for a $\mathcal{U}$-small $\mathcal{K}$-category $\underline{\mathcal{C}}$, and define $\underline{\mathcal{B}}_{u^{\prime}}$ accordingly using Proposition B.3, Proposition 9.9 literally remains true.

Example 9.11. Consider the situation of Example 9.8 and assume in particular that (9.11) is a 2-isomorphism. We do not know whether the 2-morphism (9.12) in $\mathrm{ENH}_{\mathbf{k}}$ is invertible. But if we postcompose it with $\underline{\mathbb{I}}\left(X^{\prime}\right)=\underline{\mathbb{I}}\left(X^{\prime} ; \mathcal{U}\right) \subset \underline{\mathbb{I}}\left(X^{\prime} ; \mathcal{U}^{\prime}\right)$ (which is well-defined by Proposition B.2), the 2-isomorphism (9.13) and Proposition 9.9.(c) show that this postcomposition is a 2-isomorphism

$$
\underline{\beta}^{*} \underline{\alpha}_{*} \stackrel{\sim}{\rightarrow}_{\underline{\alpha}_{*}^{\prime}}^{\underline{\beta}^{*}}: \underline{\mathbb{I}}_{\mathrm{qc}}(Y)=\underline{\mathbb{I}}_{\mathrm{qc}}(Y ; \mathcal{U}) \rightarrow \underline{\mathbb{I}}\left(X^{\prime} ; \mathcal{U}^{\prime}\right)
$$

in $\mathrm{ENH}_{\mathrm{k}}^{\mathcal{V}^{\prime}}$. It is clear that this 2-isomorphism lifts the 2-isomorphism

$$
\mathbf{L} \beta^{*} \mathbf{R} \alpha_{*} \stackrel{\sim}{\rightarrow} \mathbf{R} \alpha_{*}^{\prime} \mathbf{L} \beta^{\prime *}: \mathrm{D}_{\mathrm{qc}}(Y)=\mathrm{D}_{\mathrm{qc}}(Y, \mathcal{U}) \rightarrow \mathrm{D}\left(X^{\prime} ; \mathcal{U}^{\prime}\right)
$$

in $\operatorname{TRCAT}_{\mathbf{k}}^{\mathcal{V}^{\prime}}$. Moreover, by part (b) of Proposition 9.9, the 2-isomorphism (9.17) is represented by a roof

$$
\underline{\beta}^{*} \underline{\alpha}_{*} \stackrel{u}{\leftarrow} F \stackrel{v}{\rightarrow} \underline{\alpha}_{*}^{\prime} \underline{\beta}^{*}
$$

of objectwise homotopy equivalences in $\operatorname{DGCAT}_{\mathrm{k}}^{\mathcal{V}^{\prime}}\left(\underline{\mathbb{I}}_{\mathrm{qc}}(Y ; \mathcal{U}), \underline{\mathbb{I}}\left(X^{\prime} ; \mathcal{U}^{\prime}\right)\right)$ where $F$ is some $\mathcal{K}_{\mathfrak{U}^{\prime}}$ functor $\underline{\mathbb{I}}_{\mathrm{qc}}(Y ; \mathcal{U}) \rightarrow \underline{\mathbb{I}}\left(X^{\prime} ; \mathcal{U}^{\prime}\right)$. The induced functor $[F]:\left[\mathbb{I}_{\mathrm{qc}}(Y ; \mathcal{U})\right] \rightarrow$ $\left[\underline{\mathbb{I}}\left(X^{\prime} ; \mathcal{U}^{\prime}\right)\right]$ lands in the essential image of $\left[\mathbb{I}\left(X^{\prime} ; \mathcal{U}\right)\right] \subset\left[\mathbb{I}\left(X^{\prime} ; \mathcal{U}^{\prime}\right)\right]$.

\section{ApPendix A. Spaltenstein's RESUlts GenERAlized TO RINGED TOPOI}

We generalize some results from [Spa88] to ringed sites and ringed topoi. We use the prefix "h" (for "homotopically") instead of Spaltenstein's prefix "K", for example we say h-limp instead of K-limp. We use the notation and a few results from 4.2. 
Let $\left(\mathcal{X}, \mathcal{O}=\mathcal{O}_{\mathcal{X}}\right)$ be a ringed site. The definition of an h-injective (resp. h-flat) complex of $\mathcal{O}$-modules is well-known.

Proposition A.1 (cf. [Spa88, Prop. 5.7]). If $F \in \mathrm{C}(\mathcal{X})$ is h-flat acyclic, then $F \otimes A$ is acyclic for every $A \in \mathrm{C}(\mathcal{X})$.

Proof. Let $G \rightarrow A$ be an h-flat resolution [SP16, 06YS]. Then $F \otimes G \rightarrow F \otimes A$ is a quasi-isomorphism, and $F \otimes G$ is acyclic.

Proposition A.2 (cf. [Spa88, Prop. 5.4.(b)]). Let $\alpha:\left(\operatorname{Sh}(\mathcal{Y}), \mathcal{O}_{\mathcal{Y}}\right) \rightarrow\left(\operatorname{Sh}(\mathcal{X}), \mathcal{O}_{\mathcal{X}}\right)$ be a morphism of ringed topoi. Let $F \in \mathrm{C}(\mathcal{X})$ be h-flat. Then $\alpha^{*} F \in \mathrm{C}(\mathcal{Y})$ is h-flat. It is acyclic if $F$ is in addition acyclic.

Proof. See [SP16, 06YX] for the first claim (the assumption there that $\mathcal{Y}$ has enough points is not necessary by the comments at the beginning of [SP16, 06YV]). Let $F$ be h-flat acyclic, then $\alpha^{-1} F \in \mathrm{C}\left(\alpha^{-1} \mathcal{O}_{\mathcal{X}}\right)$ is acyclic and h-flat, by the first claim applied to the morphism $\left(\operatorname{Sh}(\mathcal{Y}), \alpha^{-1} \mathcal{O}_{\mathcal{X}}\right) \rightarrow\left(\operatorname{Sh}(\mathcal{X}), \mathcal{O}_{\mathcal{X}}\right)$ of ringed topoi. Then $\alpha^{*} F=\mathcal{O}_{\mathcal{Y}} \otimes_{\alpha^{-1} \mathcal{O}_{\mathcal{X}}} \alpha^{-1} F$ is acyclic by Proposition A.1 (and h-flat).

Write $\mathcal{P}(\mathcal{O})=\mathcal{P}\left(\mathcal{O}_{\mathcal{X}}\right)$ for the set of all bounded above complexes $E \in \mathrm{C}(\mathcal{X})$ all of whose components $E^{n}$ are direct sums of $\mathcal{O}$-modules of the form $j_{U} \mathcal{O}_{U}$, for $U \in \mathcal{X}$. Here we use the notation from 4.2.2. All objects of $\mathcal{P}(\mathcal{O})$ are h-flat [SP16, 06YQ].

Definition A.3 (cf. [Spa88, 5.11]). A complex $W$ of $\mathcal{O}$-modules is weakly hinjective if $\underline{\mathrm{C}}_{\mathcal{X}}(F, W)$ is acyclic for all h-flat acyclic objects $F$. A complex $L$ of $\mathcal{O}$-modules is h-limp if $\underline{\mathrm{C}}_{\mathcal{X}}(P, L)$ is acyclic for all acyclic $P \in \mathcal{P}(\mathcal{O})$.

We have implications h-injective $\Rightarrow$ weakly h-injective $\Rightarrow$ h-limp.

Lemma A.4. Let $U \in \mathcal{X}$. If $A \in \mathrm{C}(\mathcal{X})$ is h-limp (resp. weakly h-injective, $h$ injective), so is $j_{U}^{*} A$.

Proof. We use results from [SP16, 03DH]. Recall that there is an adjunction $\left(j_{U !}, j_{U}^{*}\right)$ of functors between $\operatorname{Mod}(\mathcal{X})$ and $\operatorname{Mod}(\mathcal{X} / U)$, and $j_{U}$ is exact. Therefore we have

$$
\underline{\mathrm{C}}_{\mathcal{X} / U}\left(T, j_{U}^{*} A\right) \cong \underline{\mathrm{C}}_{\mathcal{X}}\left(j_{U !} T, A\right)
$$

for $T \in \mathrm{C}(\mathcal{X} / U)$, and $j_{U !} T$ is acyclic if $T$ is acyclic. Hence $j_{U}^{*}$ preserves h-injectivity.

If $(V / U)$ is an object of $\mathcal{X} / U$, then $j_{U !}\left(j_{V / U !} \mathcal{O}_{V / U}\right) \cong j_{V !} \mathcal{O}_{V}$. Therefore $j_{U !}\left(\mathcal{P}\left(\mathcal{O}_{U}\right)\right) \subset$ $\mathcal{P}(\mathcal{O})$. Hence $j_{U}^{*}$ preserves h-limpness.

In order to see that $j_{U}^{*}$ preserves weak h-injectivity we need to show that $j_{U}$ ! preserves h-flatness. Recall the projection formula

$$
j_{U !}\left(F \otimes j_{U}^{*} G\right) \cong\left(j_{U !} F\right) \otimes G
$$

for all $F \in \mathrm{C}(\mathcal{X} / U)$ and $G \in \mathrm{C}(\mathcal{X})$, see [SGA4i, Exp. IV, Prop. 12.11.(b)]. Since $j_{U}^{*}$ and $j_{U}$ are exact this proves that $j_{U}$ ! preserves h-flatness. 
Proposition A.5. (a) If $T \in \mathrm{C}(\mathcal{X})$ is acyclic and $I \in \mathrm{C}(\mathcal{X})$ is h-injective, then $\mathcal{H o m}(T, I)$ is acyclic.

(b) If $F \in \mathrm{C}(\mathcal{X})$ is h-flat acyclic and $W \in \mathrm{C}(\mathcal{X})$ is weakly h-injective, then $\mathcal{H o m}(F, W)$ is acyclic.

Proof. Let $U \in \mathcal{X}$.

(a) Let $I$ be h-injective and $T$ acyclic. Then $j_{U}^{*} I$ is h-injective by Lemma A.4, and $j_{U}^{*} T$ is acyclic. Therefore $\mathcal{H o m}(T, I)(U)=\underline{\mathrm{C}}_{\mathcal{X} / U}\left(j_{U}^{*} T, j_{U}^{*} I\right)$ is acyclic. Since $U$ is arbitrary, $\mathcal{H o m}(T, I)$ is acyclic as a complex of presheaves, and a fortiori as a complex of sheaves [SP16, 03EI].

(b) Let $W$ be weakly h-injective and $F$ h-flat acyclic. Then $j_{U}^{*} W$ is weakly hinjective by Lemma A.4, and $j_{U}^{*} F$ is acyclic, and h-flat, by Proposition A.2. This implies that $\mathcal{H o m}(F, W)(U)=\underline{\mathrm{C}}_{\mathcal{X} / U}\left(j_{U}^{*} F, j_{U}^{*} W\right)$ is acyclic, and hence $\mathcal{H o m}(F, W)$ is acyclic.

Proposition A.6 (cf. [Spa88, Prop. 5.14]). If $T \in \mathrm{C}(\mathcal{X})$ is arbitrary (resp. h-flat) and $I \in \mathrm{C}(\mathcal{X})$ is h-injective, then $\mathcal{H o m}(T, I)$ is weakly h-injective (resp. is h-injective).

Proof. Let $F \in \mathrm{C}(\mathcal{X})$ be h-flat acyclic (resp. be acyclic). Then $F \otimes T$ is acyclic by Proposition A.1 (resp. because $T$ is h-flat), and hence $\underline{\mathrm{C}}_{\mathcal{X}}(F, \mathcal{H o m}(T, I)) \cong \underline{\mathrm{C}}_{\mathcal{X}}(F \otimes$ $T, I)$ is acyclic because $I$ is h-injective.

Proposition A.7 (cf. [Spa88, Prop. 5.16]). Let $A \in \mathrm{C}(\mathcal{X})$ be an h-limp acyclic complex and $U \in \mathcal{X}$. Then $\Gamma(U, A)=\Gamma\left(U, j_{U}^{*} A\right)$ is acyclic.

Proof. The proof of [Spa88, Prop. 5.16] generalizes.

Proposition A.8 (cf. [Spa88, Prop. 5.15]). Let $\alpha:\left(\mathcal{Y}, \mathcal{O}_{\mathcal{Y}}\right) \rightarrow\left(\mathcal{X}, \mathcal{O}_{\mathcal{X}}\right)$ be a morphism of ringed sites. Let $W \in \mathrm{C}(\mathcal{Y})$ be h-limp. Then $\alpha_{*} W$ is h-limp. It is acyclic if $W$ is in addition acyclic.

Proof. We claim that $\alpha^{*}\left(\mathcal{P}\left(\mathcal{O}_{\mathcal{X}}\right) \subset \mathcal{P}\left(\mathcal{O}_{\mathcal{Y}}\right)\right.$. Let $\alpha$ be given by the continuous functor $u: \mathcal{X} \rightarrow \mathcal{Y}$. Let $U \in \mathcal{X}$ and denote the induced morphism $\left(\mathcal{Y} / u(U), \mathcal{O}_{u(U)}\right) \rightarrow$ $\left(\mathcal{X} / U, \mathcal{O}_{U}\right)$ of ringed sites also by $\alpha$. Then $j_{U}^{*} \alpha_{*}=\alpha_{*} j_{u(U)}^{*}$ (see [SP16, 04IZ]) implies $\alpha^{*} j_{U !} \cong j_{u(U) !} \alpha^{*}$ using the Yoneda lemma and the obvious adjunctions. Therefore the claim follows from $\alpha^{*} \mathcal{O}_{U}=\mathcal{O}_{u(U)}$.

Let $W \in \mathrm{C}(\mathcal{Y})$ be h-limp. For $F \in \mathcal{P}\left(\mathcal{O}_{\mathcal{X}}\right)$ we have just seen that $\alpha^{*} F \in \mathcal{P}\left(\mathcal{O}_{\mathcal{Y}}\right)$. If $F$ is in addition acyclic, so is $\alpha^{*} F$ by Proposition A.2. Then $\underline{\mathrm{C}}_{\mathcal{X}}\left(F, \alpha_{*} W\right) \cong$ $\underline{\mathrm{C}}_{\mathcal{Y}}\left(\alpha^{*} F, W\right)$ is acyclic, so $\alpha_{*} W$ is h-limp.

For the second claim note that $\alpha_{*}: \mathrm{C}(\mathcal{Y}) \rightarrow \mathrm{C}(\mathcal{X})$ is given by $\left(\alpha_{*} A\right)(U)=A(u(U))=$ $\Gamma(u(U), A)$ for $A \in \mathrm{C}(\mathcal{Y})$ and $U \in \mathcal{X}$. Therefore Proposition A.7 shows that $\alpha_{*}$ maps h-limp acyclic complexes to acyclic complexes.

Proposition A.9 (cf. [Spa88, Prop. 5.15]). Let $\alpha:\left(\operatorname{Sh}(\mathcal{Y}), \mathcal{O}_{\mathcal{Y}}\right) \rightarrow\left(\operatorname{Sh}(\mathcal{X}), \mathcal{O}_{\mathcal{X}}\right)$ be a morphism of ringed topoi. Let $W \in \mathrm{C}(\mathcal{Y})$ be weakly h-injective. Then $\alpha_{*} W$ is weakly $h$-injective. It is acyclic if $W$ is in addition acyclic. 
Proof. Let $W \in \mathrm{C}(\mathcal{Y})$ be weakly h-injective. Let $F \in \mathrm{C}(\mathcal{X})$ be h-flat acyclic. Then $\alpha^{*} F$ is h-flat acyclic by Proposition A.2. Therefore $\underline{\mathrm{C}}_{\mathcal{X}}\left(F, \alpha_{*} W\right) \cong \underline{\mathrm{C}}_{\mathcal{Y}}\left(\alpha^{*} F, W\right)$ is acyclic, so $\alpha_{*} W$ is weakly h-injective.

We can factor $\alpha$ as a composition

$$
\left(\operatorname{Sh}(\mathcal{Y}), \mathcal{O}_{\mathcal{Y}}\right) \stackrel{f}{\rightarrow}\left(\operatorname{Sh}\left(\mathcal{Y}^{\prime}\right), \mathcal{O}_{\mathcal{Y}^{\prime}}\right) \stackrel{g}{\rightarrow}\left(\operatorname{Sh}(\mathcal{X}), \mathcal{O}_{\mathcal{X}}\right)
$$

of an equivalence $f$ of ringed topoi followed by a morphism of ringed topoi $g$ induced by a morphism of ringed sites, cf. [SP16, 03A2, 03CR]. If $A \in \mathrm{C}(\mathcal{Y})$ is weakly hinjective acyclic, then $f_{*} A$ is weakly h-injective acyclic, in particular h-limp acyclic, and hence $g_{*} f_{*} A=\alpha_{*} A$ is acyclic by Proposition A.8.

Proposition A.10 (cf. [Spa88, Prop. 5.20.(a)]). If $F \in \mathrm{C}(\mathcal{X})$ is $h$-flat and $W \in \mathrm{C}(\mathcal{X})$ is weakly h-injective acyclic, then $\underline{\mathrm{C}}_{\mathcal{X}}(F, W)$ is acyclic.

Proof. Let $L \in \mathrm{C}(\mathcal{X})$ be h-limp acyclic and $U \in \mathcal{X}$. Proposition A.7 shows that $\underline{\mathrm{C}}_{\mathcal{X}}\left(j_{U !} \mathcal{O}_{U}, L\right)=\underline{\mathrm{C}}_{\mathcal{X} / U}\left(\mathcal{O}_{U}, j_{U}^{*} L\right)=\Gamma(U, L)$ is acyclic. The proof of [Spa88, Prop. 5.20.(c)] then shows that $\underline{\mathrm{C}}_{\mathcal{X}}(T, L)$ is acyclic for all objects $T \in \underline{\mathcal{P}}_{\rightarrow}(\mathcal{O})$ (see [Spa88, 2.9] for the definition of $\underset{\mathcal{P}}{\rightarrow}(\mathcal{O}))$.

Let $p: P \rightarrow F$ be a quasi-isomorphism with $P \in \underline{\mathcal{P}}_{(}(\mathcal{O})$ (which exists by the proof of [Spa88, Prop. 5.6], or by [SP16, 077J]). Then $P$ and Cone $(p)$ are h-flat, and Cone $(p)$ is of course acyclic. Since $W$ is weakly h-injective, $\underline{\mathrm{C}}_{\mathcal{X}}(\mathrm{Cone}(p), W)$ is acyclic by definition, so $\underline{\mathrm{C}}_{\mathcal{X}}(F, W) \rightarrow \underline{\mathrm{C}}_{\mathcal{X}}(P, W)$ is a quasi-isomorphism whose target is acyclic by the above applied to $L=W$.

Corollary A.11. If $F \in \mathrm{C}(\mathcal{X})$ is h-flat and $W \in \mathrm{C}(\mathcal{X})$ is weakly h-injective, then $\left[\underline{\mathrm{C}}_{\mathcal{X}}\right](F, W) \rightarrow \mathrm{D}_{\mathcal{X}}(F, W)$ is an isomorphism.

Proof. Let $W \rightarrow I$ be a quasi-isomorphism with $I$ h-injective. Consider the commutative diagram

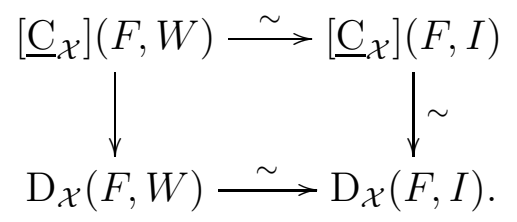

Its upper horizontal arrow is an isomorphism by Proposition A.10. The lower horizontal and the right vertical arrow are certainly isomorphisms. The claim follows.

Corollary A.12. If $F \in \mathrm{C}(\mathcal{X})$ is $h$-flat and $W \in \mathrm{C}(\mathcal{X})$ is weakly h-injective acyclic, then $\mathcal{H o m}(F, W)$ is acyclic.

Proof. Let $U \in \mathcal{X}$. Then $j_{U}^{*} W$ is weakly h-injective acyclic by Lemma A.4, and $j_{U}^{*} F$ is h-flat, by Proposition A.2. Therefore $\mathcal{H o m}(F, W)(U)=\underline{\mathrm{C}}_{\mathcal{X} / U}\left(j_{U}^{*} F, j_{U}^{*} W\right)$ is acyclic by Proposition A.10. Since $U \in \mathcal{X}$ was arbitrary, $\mathcal{H o m}(F, W)$ is acyclic. 
Proposition A.13 (cf. [Spa88, Prop. 6.1, 6.5]). Let $(\mathcal{X}, \mathcal{O})$ be a ringed site. Let $A, B \in \mathrm{D}(\mathcal{X})$.

(a) Then $\mathbf{R} \operatorname{Hom}(A, B)$ and $\mathbf{R} \mathcal{H o m}(A, B)$ are defined and can be computed by any of the following methods:

(i) using an h-injective resolution of $B$;

(ii) using an h-flat resolution of $A$ and a weakly h-injective resolution of $B$.

(b) Then $A \otimes^{\mathbf{L}} B$ is defined and can be computed using an $h$-flat resolution of $A$ or $B$.

Proof. (a).(i) Use the definition for RHom, and Proposition A.5.(a) for $\mathbf{R} \mathcal{H} o m$.

(a).(ii) Use the definition and Proposition A.10 for RHom. Use Proposition A.5.(b) and Corollary A.12 for RHom.

(b) Clear from Proposition A.1.

Proposition A.14. If $\alpha:\left(\operatorname{Sh}(\mathcal{Y}), \mathcal{O}_{\mathcal{Y}}\right) \rightarrow\left(\operatorname{Sh}(\mathcal{X}), \mathcal{O}_{\mathcal{X}}\right)$ is a morphism of ringed topoi, $\mathbf{R} \alpha_{*}$ is defined and may be computed using weakly h-injective resolutions, and $\mathbf{L} \alpha^{*}$ exists and may be computed using $h$-flat resolutions.

If $\alpha$ comes from a morphism $\left(\mathcal{Y}, \mathcal{O}_{\mathcal{Y}}\right) \rightarrow\left(\mathcal{X}, \mathcal{O}_{\mathcal{X}}\right)$ of ringed sites, $\mathbf{R} \alpha_{*}$ may even be computed using h-limp resolutions.

Proof. Certainly, $\mathbf{R} \alpha_{*}$ exists and can be computed using h-injective resolutions. Proposition A.9 shows that it can even be computed using weakly h-injective resolutions.

Certainly L $\alpha^{*}$ exists [SP16, 06YY], and Proposition A.2 shows that it can be computed using h-flat resolutions.

The last claim follows from Proposition A.8.

The usual formulas $\mathbf{R}(\alpha \beta)_{*} \cong \mathbf{R} \alpha_{*} \mathbf{R} \beta_{*}, \mathbf{L}(\alpha \beta)^{*} \cong \mathbf{L} \beta^{*} \mathbf{L} \alpha^{*}$,

$$
\begin{aligned}
\mathbf{R} \alpha_{*} \mathbf{R} \mathcal{H o m}\left(\mathbf{L} \alpha^{*}-,-\right) & \cong \mathbf{R} \mathcal{H o m}\left(-, \mathbf{R} \alpha_{*}-\right), \\
\mathbf{R H o m}\left(-\otimes^{\mathbf{L}}-,-\right) & \cong \mathbf{R} \mathcal{H o m}(-, \mathbf{R} \mathcal{H o m}(-,-)),
\end{aligned}
$$

etc. follow readily.

\section{Appendix B. Change of universe}

Let $\mathcal{U} \subset \mathcal{V}$ be universes. If $(\mathcal{X}, \mathcal{O})$ is a $\mathcal{U}$-small $\mathrm{R}$-ringed site, it is certainly expected that the derived category $\mathrm{D}(\mathcal{X} ; \mathcal{U})$ of sheaves of $\mathcal{U}$-small modules embeds fully faithfully into the corresponding derived category $\mathrm{D}(\mathcal{X} ; \mathcal{V})$ of $\mathcal{V}$-small modules. We could not find this statement in the literature (in the setting of unbounded derived categories). In the first part of this appendix we prove this and similar statements concerning h-injective and I-fibrant complexes. In the second part we provide analog statements for dg modules. 
B.1. Sheaves of modules on ringed sites. Let $\left(\mathcal{X}, \mathcal{O}_{\mathcal{X}}\right)$ be a $\mathcal{U}$-small R-ringed site. Let $\operatorname{Mod}(\mathcal{X} ; \mathcal{U})$ be the R-category of $\mathcal{U}$-small $\mathcal{O}_{\mathcal{X}}$-modules and $\mathrm{C}(\mathcal{X} ; \mathcal{U})$ the R-category of complexes of such sheaves. Previously these categories were denoted $\operatorname{Mod}(\mathcal{X})$ and $\mathrm{C}(\mathcal{X})$.

Now, using the universe $\mathcal{V}$, there is an obvious "change of universe" R-functor

$$
\operatorname{Mod}(\mathcal{X} ; \mathcal{U}) \rightarrow \operatorname{Mod}(\mathcal{X} ; \mathcal{V})
$$

Remark B.1. The change of universe functor (B.1) is fully faithful, reflects isomorphisms, preserves all $\mathcal{U}$-small limits and colimits (hence is exact), preserves injectives, and is compatible with tensor products and sheaf homomorphisms (see [SGA4ii,

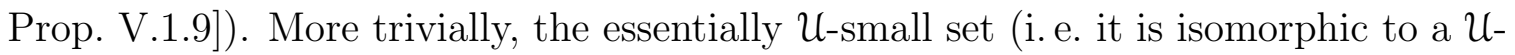
small set) of subobjects (resp. quotients) of an object of $\operatorname{Mod}(\mathcal{X} ; \mathcal{U})$ is in the obvious way identified with the set of subobjects (resp. quotients) of the corresponding object of $\operatorname{Mod}(\mathcal{X} ; \mathcal{V})$.

Proposition B.2. Let $\mathcal{U} \subset \mathcal{V}$ be universes. Let $\left(\mathcal{X}, \mathcal{O}_{\mathcal{X}}\right)$ be a $\mathcal{U}$-small $\mathrm{R}$-ringed site. Then the change of universe functor $\mathrm{C}(\mathcal{X} ; \mathcal{U}) \rightarrow \mathrm{C}(\mathcal{X} ; \mathcal{V})$ preserves h-injective complexes and $\mathbb{I}$-fibrant objects. In particular, the induced change of universe functor

$$
\mathrm{D}(\mathcal{X} ; \mathcal{U}) \rightarrow \mathrm{D}(\mathcal{X} ; \mathcal{V})
$$

on the level of derived categories is full and faithful.

Proof. We first prove the statement concerning $\mathbb{I}$-fibrant objects. Our proof is heavily based on results in $[\mathrm{KS} 06,9,14.1]$. Note that an object of $\mathrm{C}(\mathcal{X} ; \mathcal{U})$ is $\mathbb{I}$-fibrant if and only if it is QM-injective in the terminology of [KS06, Def. 9.5.1, (14.1.2)] where QM is the set of morphisms that are both quasi-isomorphisms and monomorphisms.

Let us look at the proof of [KS06, Thm. 14.1.7] in the case of the Grothendieck abelian category $\operatorname{Mod}(\mathcal{X} ; \mathcal{U})^{\mathbb{Z}}$ of graded $\mathcal{U}$-small $\mathcal{O}_{\mathcal{X}}$-modules with obvious translation. Kashiwara and Schapira show that there is an essentially $\mathcal{U}$-small full subcategory $\mathcal{S}$ of $\mathrm{C}(\mathcal{X} ; \mathcal{U})$ having the properties (i)-(iv) in [KS06, (14.1.4)] (and which can and should also be assumed to be closed under translation; a category is essentially $\mathcal{U}$-small if it is equivalent to a $\mathcal{U}$-small category). Then they show that there is a $\mathcal{U}$-small set $\mathcal{F}$ of monomorphic quasi-isomorphisms $X \rightarrow Y$ between objects $X, Y \in \mathcal{S}$ such that an object of $\mathrm{C}(\mathcal{X} ; \mathcal{U})$ is QM-injective (i. e. $\mathbb{I}$-fibrant) if and only if it is $\mathcal{F}$-injective (this statement relies on [KS06, Thm. 9.5.5]).

We need to look more closely at the construction of $\mathcal{S}$ using [KS06, 9.3, in particular Corollary 9.3.8]. Consider the generator

$$
G:=\bigoplus_{n \in \mathbb{Z}} \bigoplus_{U \in \mathcal{X}}[n] \mathrm{iCone}\left(j_{U !} \mathcal{O}_{U}\right)
$$

of $\mathrm{C}(\mathcal{X} ; \mathcal{U})$. Let $\pi_{0}$ be an infinite regular cardinal (in the universe $\mathcal{U}$ ) such that

$$
\operatorname{card}(G(G))<\pi_{0} \quad \text { and } \quad \operatorname{card}\left(G^{\oplus G(G)}(G)\right)<\pi_{0}
$$


where we abbreviate $A(B):=\mathrm{C}_{\mathcal{X}}(A, B)$; choose another infinite regular cardinal $\pi \in \mathcal{U}$ such that the conditions in $[\mathrm{KS} 06,(9.3 .4)]$ are satisfied for the generator $G$ and the category $\mathrm{C}(\mathcal{X} ; \mathcal{U})$. Then, by the proof of [KS06, Cor. 9.3.8] we can and will assume that $\mathcal{S}$ is the full subcategory $\mathrm{C}(\mathcal{X} ; \mathcal{U})_{\pi}$ of $\pi$-accessible objects in $\mathrm{C}(\mathcal{X} ; \mathcal{U})$.

Now consider the change of universe functor

$$
\varepsilon: \mathrm{C}(\mathcal{X} ; \mathcal{U}) \rightarrow \mathrm{C}(\mathcal{X} ; \mathcal{V})
$$

Let $\mathcal{S}^{\prime}:=\varepsilon(\mathcal{S})$ be the essential image of $\mathcal{S}$ under this functor. We claim that every object of $\mathcal{S}^{\prime}$ is $\pi$-accessible, i. e. $\mathcal{S}^{\prime} \subset \mathrm{C}(\mathcal{X} ; \mathcal{V})_{\pi}$.

To see this let $S \in \mathcal{S}=\mathrm{C}(\mathcal{X} ; \mathcal{U})_{\pi}$. By [KS06, Thm. 9.3.4] this means that $\operatorname{card}(S(G))<\pi$. Note that $\varepsilon(G), \pi$ and $\pi_{0}$ satisfy the conditions in [KS06, (9.3.4)] for the category $\mathrm{C}(\mathcal{X} ; \mathcal{V})$ : (a) and $(\mathrm{c})$ are obvious, (b) is satisfied by [KS06, Prop. 9.3.2] and the fact that (B.4) also holds for $G$ replaced by $\varepsilon(G)$ (by Remark B.1), and (d) is satisfied since any $\mathcal{V}$-small set $A$ with $\operatorname{card}(A)<\pi_{0}$ is isomorphic to a $\mathcal{U}$-small set $B$ since $\pi_{0} \in \mathcal{U}$. Since $\operatorname{card}(\varepsilon(S)(\varepsilon(G)))=\operatorname{card}(S(G))<\pi$ we obtain from [KS06, Thm. 9.3.4] that $\varepsilon(S)$ is $\pi$-accessible. This proves our claim that any object of $\mathcal{S}^{\prime}$ is $\pi$-accessible.

We now claim that $\mathcal{S}^{\prime}$ (which is obviously closed under translation) also has the properties (i)-(iv) in $[\mathrm{KS} 06,(14.1 .4)]$, now with respect to $\mathrm{C}(\mathcal{X} ; \mathcal{V})$ : property (i) is clear since $\varepsilon(G)$ is a generator, and properties (ii) and (iv) are obvious (cf. Remark B.1). To establish (iii) it is sufficient to check the following condition: Given any epimorphism $f: Z \rightarrow Y$ with $Y \in \mathcal{S}^{\prime}$, there is an object $S \in \mathcal{S}^{\prime}$ and a morphism $g: S \rightarrow Z$ such that the composition $f \circ g$ is an epimorphism.

To see this, we copy the argument from the proof of [KS06, Cor. 9.3.8]. Consider the epimorphisms

$$
\varepsilon(G)^{\oplus Z(\varepsilon(G))} \rightarrow Z \rightarrow Y
$$

and the $\mathcal{V}$-small $\pi$-filtered ordered set

$$
I:=\{A \subset Z(\varepsilon(G)) \mid \operatorname{card}(A)<\pi\} .
$$

Note that $\operatorname{colim}_{A \in I} \varepsilon(G)^{\oplus A} \stackrel{\sim}{\rightarrow} \varepsilon(G)^{\oplus Z(\varepsilon(G))}$. Since $Y \in \mathcal{S}^{\prime} \subset \mathrm{C}(\mathcal{X} ; \mathcal{V})_{\pi}$ there is, by [KS06, Lemma 9.3.1], an element $A \in I$ such that the composition $\varepsilon(G)^{\oplus A} \rightarrow$ $Z \rightarrow Y$ is already an epimorphism. It remains to show that $\varepsilon(G)^{\oplus A} \in \mathcal{S}^{\prime}$. Since $\operatorname{card}(A)<\pi \in \mathcal{U}$ there is a $\mathcal{U}$-small set $B$ such that $B \cong A$, and it is enough to show that $G^{\oplus B} \in \mathcal{S}=\mathrm{C}(\mathcal{X} ; \mathcal{U})_{\pi}$. By [KS06, Thm. 9.3.4] it is enough to show that $\operatorname{card}\left(G^{\oplus B}(G)\right)<\pi$; but this is true by [KS06, Lemma 9.3.3]. Now we have proven the claim that $\mathcal{S}^{\prime}$ has the properties (i)-(iv) in [KS06, (14.1.4)].

Looking at the proof of [KS06, Thm. 14.1.7] again, we see that an object of $\mathrm{C}(\mathcal{X} ; \mathcal{V})$ is QM-injective (i. e. I-fibrant) if and only if it is $\varepsilon(\mathcal{F})$-injective. Since $\varepsilon$ obviously sends $\mathcal{F}$-injective objects to $\varepsilon(\mathcal{F})$-injective objects, we deduce that $\varepsilon$ preserves $\mathbb{I}$ fibrant objects. 
Now it is easy to see all other claims. Let $E \in \mathrm{C}(\mathcal{X} ; \mathcal{U})$ be h-injective. Let $r: E \rightarrow I_{E}$ be a quasi-isomorphism with $\mathbb{I}$-fibrant $I_{E}$. Recall that the $\mathbb{I}$-fibrant objects are precisely the h-injective objects with injective components. Since $r$ is a quasiisomorphism between h-injective objects, it is invertible in the homotopy category $[\mathrm{C}(\mathcal{X} ; \mathcal{U})]$, and $\varepsilon(r)$ is invertible in $[\mathrm{C}(\mathcal{X} ; \mathcal{V})]$. By the above we know that $\varepsilon\left(I_{E}\right)$ is $\mathbb{I}$-fibrant and hence h-injective. Since h-injectivity is invariant under isomorphisms in the homotopy category, $\varepsilon(E)$ is h-injective as well.

The claim concerning derived categories follows since (B.1) is exact and fully faithful and the derived categories are equivalent to the corresponding homotopy categories of h-injectives.

B.2. Dg Modules over $\mathbf{d g}$ categories. Recall that $\mathcal{R}=\mathrm{C}(\operatorname{Mod}(\mathrm{R}))$. More precisely, we have $\mathcal{R}=\mathcal{R}_{\mathfrak{U}}=\mathrm{C}(\operatorname{Mod}(\mathrm{R} ; \mathcal{U}))$ where $\operatorname{Mod}(\mathrm{R} ; \mathcal{U})$ denotes the category of $\mathcal{U}$-small R-modules. Similarly, $\mathcal{R}_{\mathcal{V}}$ is defined using the universe $\mathcal{V}$.

Let $\underline{\mathcal{C}}$ be a $\mathcal{U}$-small $\mathcal{R}$-category. Let $\operatorname{Mod}(\underline{\mathcal{C}} ; \mathcal{U})$ be the R-category of $\underline{\mathcal{C}}$-modules (with values in $\underline{\mathcal{R}})$, i. e. of $\mathcal{R}$-functors $\underline{\mathfrak{e}}^{\text {op }} \rightarrow \underline{\mathcal{R}}$. Similarly, let $\operatorname{Mod}(\underline{\mathcal{C}} ; \mathcal{V})$ be the R-category of $\underline{\mathcal{C}}$-modules (with values in $\underline{\mathcal{R}}_{v}$ ), i. e. of $\mathcal{R}_{v}$-functors $\underline{\mathrm{C}}^{\text {op }} \rightarrow \underline{\mathcal{R}}_{v}$.

There is an obvious change of universe $\operatorname{R}$ functor $\operatorname{Mod}(\underline{\mathcal{C}} ; \mathcal{U}) \rightarrow \operatorname{Mod}(\underline{\mathcal{C}} ; \mathcal{V})$ which is fully faithful, reflects isomorphisms and preserves $\mathcal{U}$-small limits and colimits (hence is exact).

Proposition B.3. Let $\mathfrak{U} \subset \mathcal{V}$ be universes. Let $\underline{\mathcal{C}}$ be a $\mathcal{U}$-small $\mathcal{R}$-category. Then the change of universe functor $\mathbb{M o d}(\underline{\mathfrak{C}} ; \mathcal{U}) \rightarrow \operatorname{Mod}(\underline{\mathcal{e}} ; \mathcal{V})$ preserves h-injective, $\mathbb{I}$-fibrant, $h$-projective, and $\mathbb{P}$-cofibrant objects. In particular, the induced change of universe functor

$$
\mathrm{D}(\underline{\mathrm{e}} ; \mathcal{U}) \rightarrow \mathrm{D}(\underline{\mathrm{e}} ; \mathcal{V})
$$

on the level of derived categories is full and faithful.

Proof. The proof that h-injective and $\mathbb{I}$-fibrant objects are preserved follows the proof of Proposition B.2, taking the generator $G:=\bigoplus_{n \in \mathbb{Z}} \bigoplus_{C \in \underline{\mathcal{e}}}[n] \mathrm{iCone}(\operatorname{Yo}(C))$.

The proof that $\mathbb{P}$-cofibrant objects are preserved uses the fact that every $\mathbb{P}$-cofibrant object is a retract of a semi-free $\underline{\mathcal{C}}$-module, see e.g. [LS14, Lemma 2.7], and semifree $\underline{\mathcal{C}}$-modules are clearly preserved. Since all $\mathbb{P}$-cofibrant objects are h-projective, see e.g. [LS14, Lemma 2.6], the claim concerning h-projective objects follows as the corresponding claim for h-injectives in the proof of Proposition B.2.

The statement about derived categories is then obvious.

\section{Appendix C. Some Results on model Categories}

Our aim is to prove Theorem C.3. This result should be well-known to the experts and is not difficult to prove but we could not find a reference in the literature. 
As a preparation we need Proposition C.1. Its proof provides some details to the last claim in the proof of [MP12, Thm. 14.4.6], and also to the last sentence in the proof of [Hir03, Thm. 8.3.5]. We use the terminology of fibrant cofibrant and cofibrant fibrant approximations from [Hir03, Def. 8.1.2] and in particular do not assume that these approximations are functorial.

Proposition C.1 (cf. [MP12, Thm. 14.4.6]). Let $\mathcal{M}$ be a model category (with respect to some universe). For each object $M \in \mathcal{M}$ choose a fibrant cofibrant approximation $q_{M}: Q M \rightarrow M$ and a cofibrant fibrant approximation $r_{M}: M \rightarrow R M$. Let $\operatorname{Ho}(\mathcal{M})$ and $\gamma: \mathcal{M} \rightarrow \operatorname{Ho}(\mathcal{M})$ be defined as in $[\mathrm{MP} 12$, Def. 14.4.5]. Then:

(a) A morphism $m$ in $\mathcal{M}$ is a weak equivalence if and only if $\gamma(m)$ is an isomorphism.

(b) Let $f: X \rightarrow Y$ be a morphism in $\mathrm{Ho}(\mathcal{M})$. Then there is a morphism $g: R Q X \rightarrow$ $R Q Y$ such that

$$
f=\gamma\left(q_{Y}\right) \circ \gamma\left(r_{Q Y}\right)^{-1} \circ \gamma(g) \circ \gamma\left(r_{Q X}\right) \circ \gamma\left(q_{X}\right)^{-1}
$$

in $\operatorname{Ho}(\mathcal{M})$.

Proof. (a) This is the first statement of [MP12, Thm. 14.4.6].

Before proving (b) we start with an observation. Recall first that the definition of $\gamma$ uses the fact that any morphism $m: M \rightarrow N$ admits morphisms $Q m: Q M \rightarrow Q N$ and $R m: R M \rightarrow R N$ such that $q_{N} \circ Q m=m \circ q_{M}$ and $r_{N} \circ m=R m \circ r_{M}$ (cf. [MP12, 14.4]). Note however that we do not assume that $m \mapsto Q m$ or $m \mapsto R m$ respect identities or composition.

For any object $M \in \mathcal{M}$ consider the following commutative diagram in $\mathcal{M}$ which is constructed starting from its top row.

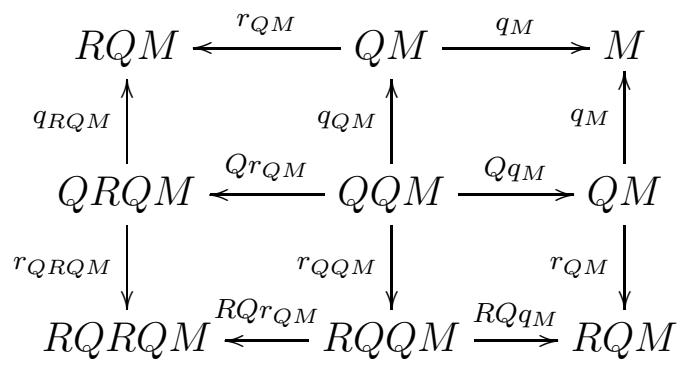

All arrows are weak equivalences by the 2-out-of-3 property. Hence $\gamma$ maps all arrows to isomorphisms. Since top row and right column of our diagram coincide, this implies that

$$
\gamma\left(R Q q_{M}\right) \circ \gamma\left(R Q r_{Q M}\right)^{-1}=\gamma\left(q_{R Q M}\right) \circ \gamma\left(r_{Q R Q M}\right)^{-1} .
$$

Let $\mathcal{M}_{\text {bifib }}$ be the full subcategory of $\mathcal{M}$ of bifibrant objects. Let $\mathrm{h}\left(\mathcal{M}_{\text {bifib }}\right)$ denote the category whose objects are the objects of $\mathcal{M}_{\text {bifib }}$ and whose morphisms are equivalence classes of morphisms up to homotopy. There is a canonical functor 
$\mathcal{M}_{\text {bifib }} \rightarrow \mathrm{h}\left(\mathcal{M}_{\text {bifib }}\right)$ which is the identity on objects and maps a morphism $m$ to its equivalence class $[m]$.

All objects in the bottom row and left column of diagram (C.2) are in $\mathcal{M}_{\text {bifib }}$, and all arrows between these objects are isomorphisms in $\mathrm{h}\left(\mathcal{M}_{\text {bifib }}\right)$, by [MP12, 14.3.15]. The functor $\gamma$ induces a functor $\mathrm{h}\left(\mathcal{M}_{\text {bifib }}\right) \rightarrow \operatorname{Ho}(\mathcal{M})$ (by [Hir03, Lemma 8.3.4]) which is easily seen to be full and faithful (and even an equivalence), cf. [Hov99, Prop. 1.2.5]. (We do not cite [Hir03, Thm. 8.3.6] or [Hov99, Thm. 1.2.10] since the result we prove is a strengthening of the last sentence in the proof of [Hir03, Thm. 8.3.5].) Therefore (C.3) implies

$$
\left[R Q q_{M}\right] \circ\left[R Q r_{Q M}\right]^{-1}=\left[q_{R Q M}\right] \circ\left[r_{Q R Q M}\right]^{-1}
$$

in $\mathrm{h}\left(\mathcal{M}_{\text {bifib }}\right)$.

(b) Let $f: X \rightarrow Y$ be an arbitrary morphism in $\operatorname{Ho}(\mathcal{M})$. Then $f$ is the homotopy class $[\hat{f}]$ of a morphism $\hat{f}: R Q X \rightarrow R Q Y$ in $\mathcal{M}_{\text {bifib }}$. We have a commutative diagram

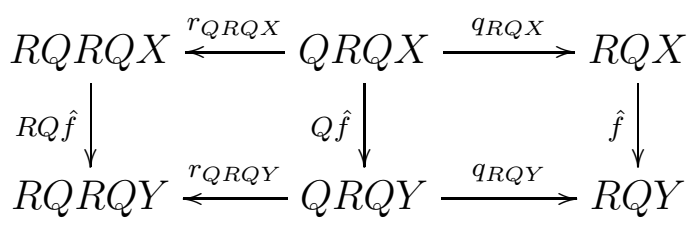

in $\mathcal{M}_{\text {bifib }}$. If we pass to $\mathrm{h}\left(\mathcal{M}_{\text {bifib }}\right)$, replace the left horizontal arrows by their inverses, and use (C.4), we see that the diagram

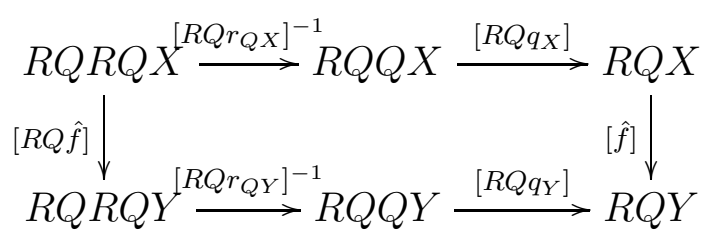

in $\mathrm{h}\left(\mathcal{M}_{\text {bifib }}\right)$ commutes. But this diagram just says that the diagram

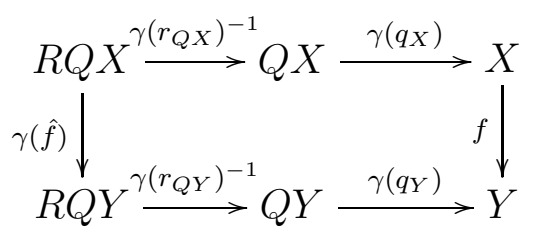

in $\operatorname{Ho}(\mathcal{M})$ commutes. This implies the proposition by setting $g=\hat{f}$.

Remark C.2. The functor $\gamma: \mathcal{M} \rightarrow \operatorname{Ho}(\mathcal{M})$ from Proposition C.1 is of course the localization of $\mathcal{M}$ with respect to the set of weak equivalences, see [Hir03, Thm. 8.3.6].

Theorem C.3. Let $\mathcal{S}$ be a full subcategory of a model category $\mathcal{M}$ with respect to some universe $\mathcal{U}$ such that each object $S$ of $\mathcal{S}$ admits a fibrant cofibrant approximation $\widetilde{S} \rightarrow S$ with $\widetilde{S} \in \mathcal{S}$ and a cofibrant fibrant approximation $S \rightarrow \hat{S}$ with $\hat{S} \in \mathcal{S}$. Let $\gamma: \mathcal{M} \rightarrow \operatorname{Ho}(\mathcal{M})$ be the canonical functor to the homotopy category of $\mathcal{M}$. Let 
$\delta: \mathcal{S} \rightarrow \mathrm{L} \mathcal{S}$ be the localization of $\mathcal{S}$ with respect to the set of weak equivalences in $\mathcal{S}$. Then

(a) we may and will assume that the category $\mathrm{LS}$ has $\mathcal{U}$-small Hom-sets, that it has the same set of objects as $\mathcal{S}$ and that $\delta$ is the identity on objects;

(b) the unique functor $\mathrm{LS} \rightarrow \operatorname{Ho}(\mathcal{M})$ whose composition with $\delta$ is the composition $\mathcal{S} \rightarrow \mathcal{M} \stackrel{\gamma}{\rightarrow} \operatorname{Ho}(\mathcal{M})$ is full and faithful;

(c) a morphism $f$ in $\mathcal{S}$ is a weak equivalence if and only if $\delta(f)$ is an isomorphism;

(d) any morphism $f$ in $\mathrm{L} \mathcal{S}$ has the form

$$
f=\delta\left(q^{\prime}\right) \circ \delta\left(r^{\prime}\right)^{-1} \circ \delta\left(f^{\prime}\right) \circ \delta(r) \circ \delta(q)^{-1}
$$

where $q, q^{\prime}, r, r^{\prime}$ are weak equivalences in $\mathcal{S}$ and $f^{\prime}$ is a morphism in $\mathcal{S}$; more precisely, $q$ and $q^{\prime}$ are trivial fibrations from a cofibrant object, and $r$ and $r^{\prime}$ are trivial cofibrations to a bifibrant object;

(e) if a morphism $f$ in $\mathrm{LS}$ is represented as in (d) then $f$ is an isomorphism if and only if $f^{\prime}$ is a weak equivalence;

(f) if all objects of $\mathcal{S}$ are fibrant, we can assume that $r=\mathrm{id}$ and $r^{\prime}=\mathrm{id}$ in (d), i. e. $f=\delta\left(q^{\prime} f^{\prime}\right) \circ \delta(q)^{-1}$.

Assume that $h: \mathcal{M} \rightarrow \mathcal{C}$ is a functor to some category $\mathcal{C}$ such that a morphism $f$ in $\mathcal{M}$ is a weak equivalence if and only if $h(f)$ is an isomorphism. Let $h_{\mathcal{S}}: \mathcal{S} \rightarrow \mathcal{C}$ be the restriction of $h$ to $\mathcal{S}$. Then

(g) the unique functor $\bar{h}_{\mathcal{S}}: \mathrm{LS} \rightarrow \mathcal{C}$ such $h_{\mathcal{S}}=\bar{h}_{\mathcal{S}} \circ \delta$ reflects isomorphisms.

Proof. We can assume that $\gamma: \mathcal{M} \rightarrow \operatorname{Ho}(\mathcal{M})$ is defined as in Proposition C.1, cf. Remark C.2. Let LS be the full subcategory of $\operatorname{Ho}(\mathcal{M})$ consisting of the objects $\gamma(S)=S$, for $S \in \mathcal{S}$, and let $\delta: \mathcal{S} \rightarrow \mathrm{L} \mathcal{S}$ be the functor induced by $\gamma$ (which will turn out to be the localization we want).

(c) and (d): This follows from Proposition C.1 and our assumption that the fibrant cofibrant (resp. cofibrant fibrant) approximations of objects of $\mathcal{S}$ exist in $\mathcal{S}$.

(a) This and the fact that $\delta$ is the localization with respect to the set of weak equivalences follows from the proof of [Hir03, Thm. 8.3.6] because (d) is known.

(b) Obvious by construction.

(e) Clear from (c).

(f) Clear since all cofibrant fibrant approximations of objects of $\mathcal{S}$ can be taken to be the identities.

(g) The functor $\bar{h}_{\mathcal{S}}$ exists uniquely by the universal property of the localization. Let $f$ be a morphism in $\mathrm{LS}$ such that $\bar{h}_{\mathcal{S}}(f)$ is an isomorphism. Represent $f$ as in (d). Then $\bar{h}_{\mathcal{S}}(f)=h\left(q^{\prime}\right) \circ h\left(r^{\prime}\right)^{-1} \circ h\left(f^{\prime}\right) \circ h(r) \circ h(q)^{-1}$, and $h(q), h\left(q^{\prime}\right), h(r), h\left(r^{\prime}\right)$ are isomorphisms by assumption. Therefore $h\left(f^{\prime}\right)$ is an isomorphism, so $f^{\prime}$ is a weak equivalence, and (e) shows that $f$ is an isomorphism. 


\section{REFERENCES}

[Bek00] Tibor Beke. Sheafifiable homotopy model categories. Math. Proc. Cambridge Philos. Soc., 129(3):447-475, 2000.

[BK90] A. I. Bondal and M. M. Kapranov. Enhanced triangulated categories. Mat. Sb., 181(5):669-683, 1990.

[BLL04] Alexey I. Bondal, Michael Larsen, and Valery A. Lunts. Grothendieck ring of pretriangulated categories. Int. Math. Res. Not., (29):1461-1495, 2004.

[Bor94] Francis Borceux. Handbook of categorical algebra. 1, volume 50 of Encyclopedia of Mathematics and its Applications. Cambridge University Press, Cambridge, 1994.

[CD09] Denis-Charles Cisinski and Frédéric Déglise. Local and stable homological algebra in Grothendieck abelian categories. Homology, Homotopy Appl., 11(1):219-260, 2009.

[Cir17] Emanuel Rodríguez Cirone. A strictly-functorial and small dg-enhancement of the derived category of perfect complexes. Preprint, 2017. arXiv:1502.06573v2.

[Cis10] Denis-Charles Cisinski. Localizing an arbitrary additive category. MathOverflow, 2010. http://mathoverflow.net/q/44155 (version: 2010-10-29).

[DHI04] Daniel Dugger, Sharon Hollander, and Daniel C. Isaksen. Hypercovers and simplicial presheaves. Math. Proc. Cambridge Philos. Soc., 136(1):9-51, 2004.

[Dri08] Vladimir Drinfeld. DG quotients of DG categories. Revised Publication, 2008. arXiv:math/0210114v7.

[Gil06] James Gillespie. The flat model structure on complexes of sheaves. Trans. Amer. Math. Soc., 358(7):2855-2874, 2006.

[Gil07] James Gillespie. Kaplansky classes and derived categories. Math. Z., 257(4):811-843, 2007.

[GK08] Nora Ganter and Mikhail Kapranov. Representation and character theory in 2-categories. Adv. Math., 217(5):2268-2300, 2008.

[GR17] Ofer Gabber and Lorenzo Ramero. Foundations for almost ring theory. Preprint, Release 7, 2017. arXiv:math/0409584v12.

[Gro12] Moritz Groth. Monoidal derivators and additive derivators. Preprint, 2012. arXiv:1203.5071v1.

[Gui11] Stéphane Guillermou. dg-Methods for Microlocalization. Publ. Res. Inst. Math. Sci., 47(1):99-140, 2011.

[Hir03] Philip S. Hirschhorn. Model categories and their localizations, volume 99 of Mathematical Surveys and Monographs. American Mathematical Society, Providence, RI, 2003.

[Hör17] Fritz Hörmann. Six functor formalisms and fibered multiderivators. Preprint, 2017. arXiv:1603.02146v2.

[Hov99] Mark Hovey. Model categories, volume 63 of Mathematical Surveys and Monographs. American Mathematical Society, Providence, RI, 1999.

[Kel05] G. M. Kelly. Basic concepts of enriched category theory. Repr. Theory Appl. Categ., (10):vi+137, 2005.

[Kel06] Bernhard Keller. On differential graded categories. In International Congress of Mathematicians. Vol. II, pages 151-190. Eur. Math. Soc., Zürich, 2006.

[KS94] Masaki Kashiwara and Pierre Schapira. Sheaves on manifolds, volume 292 of Grundlehren der Mathematischen Wissenschaften. Springer-Verlag, Berlin, 1994.

[KS06] Masaki Kashiwara and Pierre Schapira. Categories and sheaves, volume 332 of Grundlehren der Mathematischen Wissenschaften. Springer-Verlag, Berlin, 2006.

[Kuz15] Alexander Kuznetsov. Height of exceptional collections and Hochschild cohomology of quasiphantom categories. J. Reine Angew. Math., 708:213-243, 2015. 
[Law16] Tyler Lawson. Localization of enriched categories and cubical sets. Preprint, 2016. arXiv:1602.05313v1.

[Lei04] Tom Leinster. Higher operads, higher categories, volume 298 of London Mathematical Society Lecture Note Series. Cambridge University Press, Cambridge, 2004.

[Lip09] Joseph Lipman. Notes on derived functors and Grothendieck duality. In Foundations of Grothendieck duality for diagrams of schemes, volume 1960 of Lecture Notes in Math., pages 1-259. Springer, Berlin, 2009.

[LO10] Valery A. Lunts and Dmitri O. Orlov. Uniqueness of enhancement for triangulated categories. J. Amer. Math. Soc., 23(3):853-908, 2010.

[LS14] Valery A. Lunts and Olaf M. Schnürer. Smoothness of equivariant derived categories. Proc. Lond. Math. Soc. (3), 108(5):1226-1276, 2014.

[LS16] Valery A. Lunts and Olaf M. Schnürer. New enhancements of derived categories of coherent sheaves and applications. J. Algebra, 446:203-274, 2016.

[Lun10] Valery A. Lunts. Categorical resolution of singularities. J. Algebra, 323(10):2977-3003, 2010.

[Lur09] Jacob Lurie. Higher topos theory, volume 170 of Annals of Mathematics Studies. Princeton University Press, Princeton, NJ, 2009.

[LZ17] Yifeng Liu and Weizhe Zheng. Enhanced six operations and base change theorem for Artin stacks. Preprint, 2017. arXiv:1211.5948v3.

[ML98] Saunders Mac Lane. Categories for the working mathematician, volume 5 of Graduate Texts in Mathematics. Springer-Verlag, New York, second edition, 1998.

[MP12] J. P. May and K. Ponto. More concise algebraic topology. Chicago Lectures in Mathematics. University of Chicago Press, Chicago, IL, 2012. Localization, completion, and model categories.

[Nad09] David Nadler. Microlocal branes are constructible sheaves. Selecta Math. (N.S.), 15(4):563-619, 2009.

[PvdB17] Alexander Polishchuk and Michel van den Bergh. Semiorthogonal decompositions of the categories of equivariant coherent sheaves for some reflection groups. Preprint, 2017. arXiv:1503.04160v4.

[Rie14] Emily Riehl. Categorical homotopy theory, volume 24 of New Mathematical Monographs. Cambridge University Press, Cambridge, 2014.

[Sch16] Olaf M. Schnürer. Six operations on dg enhancements of derived categories of sheaves and applications. In Opening Perspectives in Algebra, Representations, and Topology (Barcelona, 2015), Trends in Mathematics. Birkhäuser Verlag, Basel, 2016. To appear.

[SGA4i] Théorie des topos et cohomologie étale des schémas. Tome 1: Théorie des topos. Lecture Notes in Mathematics, Vol. 269. Springer-Verlag, Berlin-New York, 1972. Séminaire de Géométrie Algébrique du Bois-Marie 1963-1964 (SGA 4), Dirigé par M. Artin, A. Grothendieck, et J. L. Verdier. Avec la collaboration de N. Bourbaki, P. Deligne et B. Saint-Donat.

[SGA4ii] Théorie des topos et cohomologie étale des schémas. Tome 2. Lecture Notes in Mathematics, Vol. 270. Springer-Verlag, Berlin-New York, 1972. Séminaire de Géométrie Algébrique du Bois-Marie 1963-1964 (SGA 4), Dirigé par M. Artin, A. Grothendieck et J. L. Verdier. Avec la collaboration de N. Bourbaki, P. Deligne et B. Saint-Donat.

[Shu09] Michael Shulman. Homotopy limits and colimits and enriched homotopy theory. Preprint, 2009. arXiv:math/0610194v3.

[SP16] The Stacks Project Authors. The Stacks Project. http://stacks.math.columbia.edu, 2016. 
[Spa88] N. Spaltenstein. Resolutions of unbounded complexes. Compositio Math., 65(2):121-154, 1988.

[SS16] Olaf M. Schnürer and Wolfgang Soergel. Proper base change for separated locally proper maps. Rend. Semin. Mat. Univ. Padova, 135:223-250, 2016.

[Toë07] Bertrand Toën. The homotopy theory of $d g$-categories and derived Morita theory. Invent. Math., 167(3):615-667, 2007.

[Ver66] Jean-Louis Verdier. Dualité dans la cohomologie des espaces localement compacts, Exp. No. 300. In Séminaire Bourbaki: Vol. 1965/1966, Exposés 295-312, pages viii+293. W. A. Benjamin, Inc., New York-Amsterdam, 1966.

[Wei89] Charles A. Weibel. Homotopy algebraic $K$-theory. In Algebraic $K$-theory and algebraic number theory (Honolulu, HI, 1987), volume 83 of Contemp. Math., pages 461-488. Amer. Math. Soc., Providence, RI, 1989.

Mathematisches Institut, Universität Bonn, Endenicher Allee 60, 53115 Bonn, Germany

E-mail address: olaf.schnuerer@math.uni-bonn.de 